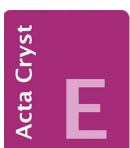

CRYSTALLOGRAPHIC COMMUNICATIONS

ISSN 2056-9890

\section{A resonance-assisted intramolecular hydrogen bond in compounds containing 2-hydroxy-3,5-dinitro- benzoic acid and its various deprotonated forms: redetermination of several related structures}

\author{
Jan Fábry*
}

Institute of Physics, Czech Academy of Sciences, Na Slovance 2, 18221 Praha 8, Czech Republic. *Correspondence e-mail: fabry@fzu.cz

A large number of structural determinations of compounds containing 2-hydroxy-3,5-dinitrobenzoic acid (I) and its various deprotonated forms, 2-hydroxy-3,5-dinitrobenzoate (II) or 2-carboxy-4,6-dinitrophenolate (III), are biased. The reason for the bias follows from incorrectly applied constraints or restraints on the bridging hydrogen, which is involved in the intramolecular hydrogen bond between the neighbouring carboxylic/carboxylate and oxo/ hydroxy groups. This hydrogen bond belongs to the category of resonanceassisted hydrogen bonds. The present article suggests corrections for the following structure determinations that have been published in Acta Crystallographica: DUJZAK, JEVNAA, LUDFUL, NUQVEB, QIQJAD, SAFGUD, SEDKET, TIYZIM, TUJPEV, VABZIJ, WADXOR, YAXPOE [refcodes are taken from the Cambridge Structural Database [CSD; Groom et al. (2016). Acta Cryst. B72, 171-179]. The structural features of the title molecules in all the retrieved structures, together with structures that contain 3,5-dinitro-2oxidobenzoate (IV), are discussed. Attention is paid to the localization of the above-mentioned bridging hydrogen, which can be situated closer to the $\mathrm{O}$ atom of the carboxylate/carboxylic group or that of the hydroxy/oxo group. In some cases, it is disordered between the two $\mathrm{O}$ atoms. The position of the bridging hydrogen seems to be dependent on the $\mathrm{p} K_{a}$ (base) although with exceptions. A stronger basicity enhances the probability of the presence of a phenolate (III). The present article examines the problem of the refinement of such a bridging hydrogen as well as that of the hydrogen atoms involved in the hydroxy and primary and secondary amine groups. It appears that the best model, in many cases, is obtained by fixing the hydrogen-atom position found in the difference electron-density map while refining its isotropic displacement parameter.

\section{Chemical context}

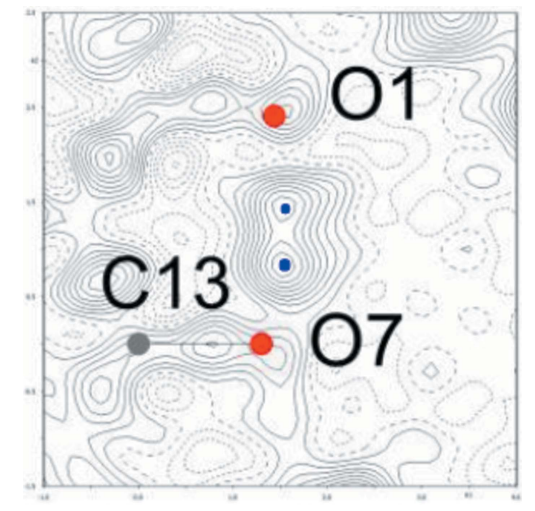

2-Hydroxy-3,5-dinitrobenzoic acid (I; alternatively 3,5-dinitrosalicylic acid, DNSA), 2-hydroxy-3,5-dinitrobenzoate (II; alternatively 3,5-dinitrosalicylate), 2-carboxy-4,6-dinitrophenolate (III) and 3,5-dinitro-2-oxidobenzoate (IV), are molecules that have interesting structural and chemical features. Such molecules have been studied because of the proton transfer from the carboxylic group, which is dependent on its environment (e.g. Smith et al., 2007). Thus, three deprotonated forms of molecule $\mathbf{I}$ have been observed. The last one, $\mathbf{I V}$, is deprived of all of the hydrogen atoms while the others differ in the localization of the hydrogen atom involved in the intramolecular hydrogen bond between the $\mathrm{O}$ atoms of the carboxylate/carboxylic and the hydroxy/oxo groups. In the different structures, this hydrogen atom may be closer to 
either oxygen atom, depending on the properties of each particular structure. In some cases, this hydrogen atom may even be disordered. In the following, it will be referred to as a bridging hydrogen.<smiles>O=[N+]([O-])c1cc2c(c([N+](=O)[O-])c1)OPO2</smiles><smiles>O=C1O[CH]Oc2c1cc([N+](=O)[O-])cc2[N+](=O)[O-]</smiles><smiles></smiles>

III

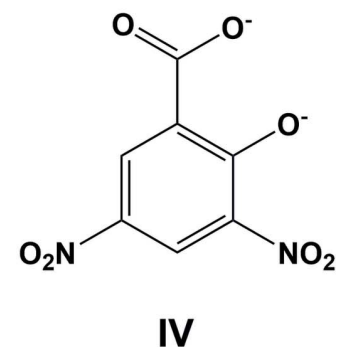

Such a bridging hydrogen is a part of a resonance-assisted moiety (Gilli \& Gilli, 2009) composed of six atoms with the pertinent bonds being $D 1, D 2, D 3, D 4, D 11$ and $D 12$, as shown in Fig. 1a. However, the delocalized bonds can be further extended within the molecule, especially to the $\mathrm{C}=\mathrm{O} / \mathrm{C}-\mathrm{OH}$ bond (D1/D5 in Fig. 1a). Resonance-assisted hydrogen bonds tend to be stronger and therefore the bridging hydrogen should be displaced towards the hydrogen-bond centre. On the other hand, $\mathrm{O} \cdots \mathrm{H} \cdots \mathrm{O}$ hydrogen bonds with a bridging hydrogen that is situated about its centre are usually observed for strong intramolecular hydrogen bonds with the $\mathrm{O} \cdots \mathrm{O}$ distances being shorter than $2.5 \AA$ (Gilli \& Gilli, 2009), while the $\mathrm{O} \cdots \mathrm{H} \cdots \mathrm{O}$ angles tend to be close to $180^{\circ}$ (Jeffrey, 1995). The $\mathrm{O}_{\text {carboxylate/carboxylicgroup }} \cdots \mathrm{O}_{\text {hydroxy/oxo group }}$ distance can be as short as $2.41 \AA$ in some 2-hydroxy-3,5-dinitrobenzoates (II) or 2-carboxy-4,6-dinitrophenolates (III); however, the $\mathrm{O} \cdots \mathrm{H} \cdots \mathrm{O}$ angle, which is $c a 160^{\circ}$, situates it in a category of its own.

The above-mentioned features of the intramolecular $\mathrm{O} \cdots \mathrm{H} \cdots \mathrm{O}$ hydrogen bond in the molecules considered herein have been ignored on many occasions by incorrectly applied constraints or severe restraints on the $\mathrm{O}-\mathrm{H}$ distances, 0.82 or $0.84 \AA$, together with angle constraints/restraints equal to $109^{\circ}$ as proposed by SHELXL (Sheldrick, 2008, 2015).

A robust indication whether the bridging hydrogen has been positioned correctly follows from the bond distances $\mathrm{C}=\mathrm{O} / \mathrm{C}-\mathrm{O}$ of the involved carboxylate/carboxylic and hydroxyl/oxo groups, although there are a few exceptions in which the bridging hydrogen is attached to the oxygen forming a slightly shorter $\mathrm{C}-\mathrm{O}$ distance. These exceptions will be mentioned briefly below. Thus, it seems that a considerable number of the structures containing the molecules I-IV could have been determined more correctly with a more realistic description of the pertinent hydrogen bond in these molecular fragments.

A search of the Cambridge Structural Database (CSD, Version 3.58, last update May 2017; Groom et al., 2016) indicated that 27 structures out of 53 reported as 2-hydroxy-3,5dinitrobenzoates (II) seem to be suspect; 21 structures out of 70 reported as 2-carboxy-4,6-dinitrophenolates (III) seem to be suspect, and nine structures out of 15 that contain a molecule of 2-hydroxy-3,5-dinitrobenzoic acid (I) also appear to be suspect. Figs. $2 a$ and $2 b$ illustrate this situation for 2-hydroxy-3,5-dinitrobenzoates (II) and 2-carboxy-4,6-dinitrophenolates (III), respectively.

It is plausible to expect that the environment affects the position of the bridging hydrogen. Therefore, it can be assumed that the proton transfer stemming from the carboxyl group will affect its position.

The data for the suspect structures published in Acta Crystallographica were retrieved from the journal's web page
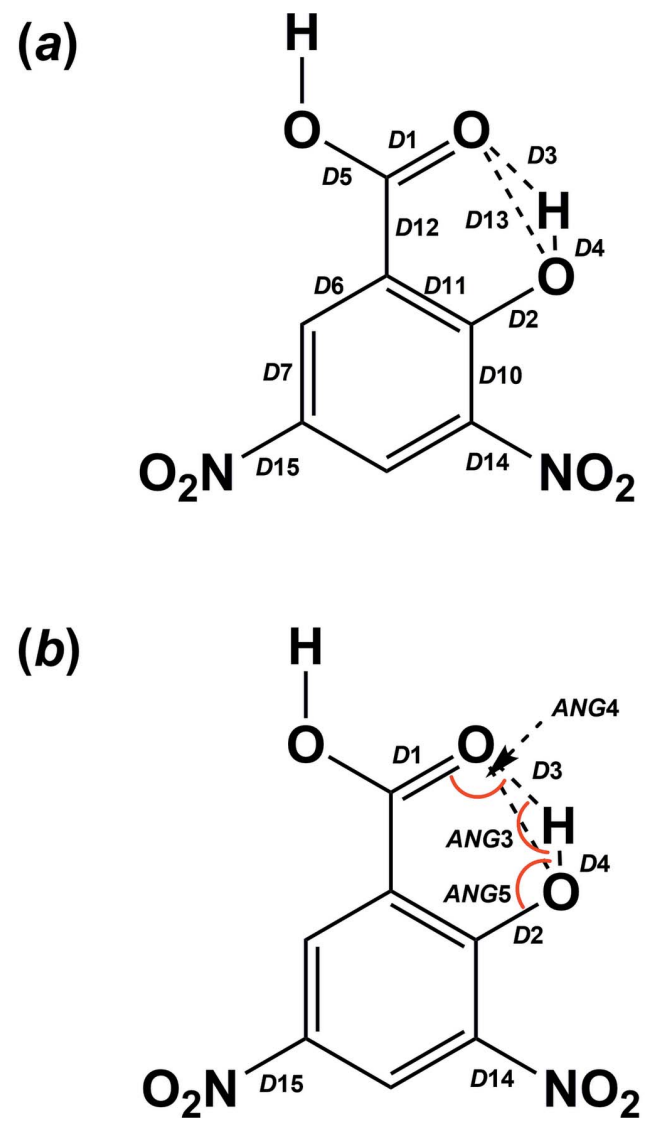

$A N G 1$ - dihedral angle between plane of $\mathrm{NO}_{2}$ group (D14) and the benzene ring $A N G 2$ - dihedral angle between plane of $\mathrm{NO}_{2}$ group (D15) and the benzene ring

Figure 1

Definition of bonds and various angles in I-IV. 
Table 1

Experimental details.

\begin{tabular}{|c|c|c|c|c|}
\hline & DUJZAK & JEVNAA & LUDFUL & NUQVEB \\
\hline \multicolumn{5}{|l|}{ Crystal data } \\
\hline Chemical formula & {$\left[\mathrm{Ag}\left(\mathrm{C}_{9} \mathrm{H}_{7} \mathrm{NO}\right)_{2}\right]\left(\mathrm{C}_{7} \mathrm{H}_{3} \mathrm{~N}_{2} \mathrm{O}_{7}\right)$} & {$\left[\mathrm{Zn}\left(\mathrm{C}_{3} \mathrm{H}_{4} \mathrm{~N}_{2}\right)_{4}\right]\left(\mathrm{C}_{7} \mathrm{H}_{3} \mathrm{~N}_{2} \mathrm{O}_{7}\right)_{2}$} & $\mathrm{C}_{7} \mathrm{H}_{4} \mathrm{~N}_{2} \mathrm{O}_{7} \cdot \mathrm{C}_{12} \mathrm{H}_{8} \mathrm{~N}_{2}$ & $\mathrm{C}_{6} \mathrm{H}_{9} \mathrm{~N}_{2}^{+} \cdot \mathrm{C}_{7} \mathrm{H}_{3} \mathrm{~N}_{2} \mathrm{O}_{7}^{-}$ \\
\hline$M_{\mathrm{r}}$ & 625.30 & 791.93 & 408.33 & 336.27 \\
\hline Crystal system, space group & Monoclinic, $P 2_{1}$ & Monoclinic, $C 2 / c$ & Monoclinic, $P 2_{1} / a$ & Triclinic, $P \overline{1}$ \\
\hline Temperature $(\mathrm{K})$ & 293 & 293 & 293 & 100 \\
\hline$a, b, c(\AA)$ & $\begin{array}{l}9.0154(18), 7.6122(15), \\
17.138(3)\end{array}$ & $\begin{array}{l}25.0809(15), 6.7251(4), \\
18.9145(10)\end{array}$ & $\begin{array}{l}14.8002(15), 7.4029(16), \\
16.0091(16)\end{array}$ & $\begin{array}{l}5.8673(7), 8.0991(9) \\
15.2437(17)\end{array}$ \\
\hline$\alpha, \beta, \gamma\left({ }^{\circ}\right)$ & $90,104.38(3), 90$ & $90,97.658(6), 90$ & $90,96.395(8), 90$ & $\begin{array}{l}86.844(3), 84.252(3) \\
81.209(3)\end{array}$ \\
\hline$V\left(\AA^{3}\right)$ & $1139.3(4)$ & $3161.9(3)$ & $1743.1(5)$ & $711.69(14)$ \\
\hline$Z$ & 2 & 4 & 4 & 2 \\
\hline Radiation type & Mo $K \alpha$ & Mo $K \alpha$ & Mo $K \alpha$ & Mo $K \alpha$ \\
\hline$\mu\left(\mathrm{mm}^{-1}\right)$ & 0.95 & 0.87 & 0.12 & 0.13 \\
\hline Crystal size (mm) & $0.20 \times 0.15 \times 0.11$ & $0.20 \times 0.18 \times 0.10$ & $0.36 \times 0.34 \times 0.26$ & $0.29 \times 0.14 \times 0.08$ \\
\hline \multicolumn{5}{|l|}{ Data collection } \\
\hline Diffractometer & $\begin{array}{l}\text { Bruker SMART CCD area- } \\
\text { detector }\end{array}$ & Bruker APEXII area-detector & Enraf-Nonius CAD-4 & $\begin{array}{l}\text { Bruker APEX DUO CCD } \\
\text { area-detector }\end{array}$ \\
\hline Absorption correction & - & $\begin{array}{l}\text { Multi-scan (SADABS; } \\
\text { Bruker, 1999) }\end{array}$ & - & $\begin{array}{l}\text { Multi-scan (SADABS; } \\
\text { Bruker, 2009) }\end{array}$ \\
\hline$T_{\min }, T_{\max }$ & - & $0.846,0.918$ & - & $0.963,0.990$ \\
\hline $\begin{array}{l}\text { No. of measured, independent } \\
\text { and observed }[I>3 \sigma(I)] \\
\text { reflections }\end{array}$ & $10841,4602,4225$ & $20634,3635,2152$ & $8396,4202,1587$ & $12709,4943,3677$ \\
\hline$R_{\text {int }}$ & 0.022 & 0.058 & 0.056 & 0.023 \\
\hline$(\sin \theta / \lambda)_{\max }\left(\AA^{-1}\right)$ & 0.651 & 0.651 & 0.661 & 0.756 \\
\hline \multicolumn{5}{|l|}{ Refinement } \\
\hline$R$ factors and goodness of fit & $\begin{array}{l}R[F>3 \sigma(F)]=0.023 \\
\quad w R(F)=0.053, S=1.34\end{array}$ & $\begin{array}{l}R[F>3 \sigma(F)]=0.036 \\
\quad w R(F)=0.075, S=1.23\end{array}$ & $\begin{array}{l}R[F>3 \sigma(F)]=0.044 \\
\quad w R(F)=0.083, S=1.08\end{array}$ & $\begin{array}{l}R[F>3 \sigma(F)]=0.042 \\
\quad w R(F)=0.109, S=2.06\end{array}$ \\
\hline No. of reflections & 4602 & 3635 & 4202 & 4943 \\
\hline No. of parameters & 356 & 244 & 274 & 222 \\
\hline No. of restraints & 0 & 0 & 0 & 0 \\
\hline $\mathrm{H}$-atom treatment & $\begin{array}{l}\text { H atoms treated by a } \\
\text { mixture of independent and } \\
\text { constrained refinement }\end{array}$ & $\begin{array}{l}\mathrm{H} \text { atoms treated by a } \\
\text { mixture of independent and } \\
\text { constrained refinement }\end{array}$ & $\begin{array}{l}\text { H atoms treated by a } \\
\text { mixture of independent and } \\
\text { constrained refinement }\end{array}$ & $\begin{array}{l}\mathrm{H} \text { atoms treated by a } \\
\text { mixture of independent } \\
\text { and constrained } \\
\text { refinement }\end{array}$ \\
\hline$\Delta \rho_{\max }, \Delta \rho_{\min }\left(\mathrm{e} \AA^{-3}\right)$ & $0.44,-0.30$ & $0.23,-0.23$ & $0.29,-0.31$ & $0.40,-0.32$ \\
\hline Absolute structure & $\begin{array}{l}1800 \text { of Friedel pairs used in } \\
\text { the refinement }\end{array}$ & - & - & - \\
\hline Absolute structure parameter & $0.004(17)$ & - & - & - \\
\hline & QIQJAD & SAFGUD & SEDKET & TIYZIM \\
\hline
\end{tabular}

Crystal data

Chemical formula

$M_{\text {r }}$

Crystal system, space group

Temperature (K)

$a, b, c(\AA)$

$\alpha, \beta, \gamma\left(^{\circ}\right)$

$V\left(\AA^{3}\right)$

Z

Radiation type

$\mu\left(\mathrm{mm}^{-1}\right)$

Crystal size (mm)

Data collection

Diffractometer

Absorption correction

$T_{\min }, T_{\max }$

No. of measured, independent and observed $[I>3 \sigma(I)]$ reflections

\section{$\mathrm{C}_{9} \mathrm{H}_{8} \mathrm{Cl}_{2} \mathrm{~N}_{5}{ }^{+} \cdot \mathrm{C}_{7} \mathrm{H}_{3} \mathrm{~N}_{2} \mathrm{O}_{7}{ }^{-} \cdot-$ $\mathrm{C}_{3} \mathrm{H}_{7} \mathrm{NO}$}

557.31

Triclinic, $P \overline{1}$

294

10.0227 (5), 10.5507 (5), $12.5359(6)$

81.858 (1), 71.888 (1), 70.009 (1)

$1183.1(1)$

2

Mo $K \alpha$

0.34

$0.16 \times 0.14 \times 0.08$

Bruker SMART APEX CCD area-detector

Multi-scan ( $S A D A B S$;

Bruker, 2001)

$0.93,0.97$

13936, 5507, 4441
$\left[\mathrm{Ag}\left(\mathrm{C}_{12} \mathrm{H}_{6} \mathrm{~N}_{2} \mathrm{O}_{2}\right)\right]\left(\mathrm{C}_{7} \mathrm{H}_{3} \mathrm{~N}_{2} \mathrm{O}_{7}\right)$

755.36

Monoclinic, $P 2_{1} / c$

174

11.757 (2), 18.297 (4), 13.223 (3)

90, 103.91 (3), 90

2761.1 (11)

4

Mo $K \alpha$

0.81

$0.3 \times 0.24 \times 0.2$

\section{$\mathrm{C}_{5} \mathrm{H}_{9} \mathrm{~N}_{2}{ }^{+} \cdot \mathrm{C}_{7} \mathrm{H}_{3} \mathrm{~N}_{2} \mathrm{O}_{7}{ }^{-}$}

324.26

Monoclinic, $P 2_{1}$

293

8.1183 (7), 6.0636 (5),

14.1453 (11)

90, 91.904 (1), 90

695.93 (10)

2

Mo $K \alpha$

0.13

$0.40 \times 0.27 \times 0.11$

Oxford Diffraction Gemini R Bruker SMART CCD Ultra

Multi-scan ( $S A D A B S$;

Bruker, 2002)

$0.780,0.910$

$12726,5013,3100$
$0.959,0.986$

$3523,2301,1444$
Multi-scan ( $S A D A B S$; Bruker, 2002)

4

$\mathrm{C}_{6} \mathrm{H}_{12} \mathrm{~N}_{3}{ }^{+} \cdot \mathrm{C}_{7} \mathrm{H}_{3} \mathrm{~N}_{2} \mathrm{O}_{7}{ }^{-}$

353.30

Triclinic, $P \overline{1}$

173

7.0109 (4), 10.6617 (8), $10.7454(7)$

93.075 (6), 95.863 (5), 104.944 (6)

$769.30(9)$

2

$\mathrm{Cu} K \alpha$

1.09

$0.22 \times 0.14 \times 0.12$
Agilent Xcalibur (Eos, Gemini)

Multi-scan (CrysAlis PRO and CrysAlis RED; Agilent, 2012)

$0.925,1.000$

4664, 2953, 2426

\author{
$\mathrm{C}_{6} \mathrm{H}_{9}{ }^{+} \cdot \mathrm{C}_{7} \mathrm{H}_{3} \mathrm{~N}_{2} \mathrm{O}_{7}{ }^{-}$ \\ Triclinic, $P \overline{1}$ \\ $5.2437(17)$ \\ $.13 \times 0.14 \times 0.08$
}

area-detector

er, 2009

$w R(F)=0.109, S=2.06$ 
Table 1 (continued)

\begin{tabular}{|c|c|c|c|c|}
\hline & QIQJAD & SAFGUD & SEDKET & TIYZIM \\
\hline$R_{\text {int }}$ & 0.019 & 0.052 & 0.040 & 0.026 \\
\hline$(\sin \theta / \lambda)_{\max }\left(\AA^{-1}\right)$ & 0.661 & 0.603 & 0.595 & 0.618 \\
\hline \multicolumn{5}{|l|}{ Refinement } \\
\hline$R$ factors and goodness of fit & $\begin{array}{l}R[F>3 \sigma(F)]=0.056 \\
\quad w R(F)=0.147, S=3.41\end{array}$ & $\begin{array}{l}R[F>3 \sigma(F)]=0.062 \\
\quad w R(F)=0.118, S=1.64\end{array}$ & $\begin{array}{l}R[F>3 \sigma(F)]=0.041 \\
\quad w R(F)=0.088, S=1.16\end{array}$ & $\begin{array}{l}R[F>3 \sigma(F)]=0.041 \\
\quad w R(F)=0.100, S=1.64\end{array}$ \\
\hline No. of reflections & 5507 & 5013 & 2301 & 2953 \\
\hline No. of parameters & 340 & 444 & 212 & 229 \\
\hline No. of restraints & 0 & 0 & 0 & 0 \\
\hline H-atom treatment & $\begin{array}{l}\text { H atoms treated by a } \\
\text { mixture of independent and } \\
\text { constrained refinement }\end{array}$ & $\begin{array}{l}\text { H-atom parameters } \\
\text { constrained }\end{array}$ & $\begin{array}{l}\text { H atoms treated by a } \\
\text { mixture of independent and } \\
\text { constrained refinement }\end{array}$ & $\begin{array}{l}\mathrm{H} \text { atoms treated by a } \\
\text { mixture of independent } \\
\text { and constrained } \\
\text { refinement }\end{array}$ \\
\hline$\Delta \rho_{\max }, \Delta \rho_{\min }\left(\mathrm{e} \AA^{-3}\right)$ & $0.80,-0.36$ & $0.76,-0.63$ & $0.11,-0.10$ & $0.21,-0.18$ \\
\hline Absolute structure & - & - & $\begin{array}{l}955 \text { Friedel pairs used in the } \\
\text { refinement }\end{array}$ & - \\
\hline \multirow[t]{2}{*}{ Absolute structure parameter } & - & - & 0.5 & - \\
\hline & TUJPEV & (VABZIJ) & WADXOR & YAXPOE \\
\hline \multicolumn{5}{|l|}{ Crystal data } \\
\hline Chemical formula & $\mathrm{C}_{10} \mathrm{H}_{12} \mathrm{~N}_{3} \mathrm{O}_{3} \mathrm{~S}^{+} \cdot \mathrm{C}_{7} \mathrm{H}_{3} \mathrm{~N}_{2} \mathrm{O}_{7}^{-}$ & $\mathrm{C}_{8} \mathrm{H}_{13} \mathrm{~N}_{2} \mathrm{O}^{+} \cdot \mathrm{C}_{7} \mathrm{H}_{3} \mathrm{~N}_{2} \mathrm{O}_{7}^{-} \cdot \mathrm{H}_{2} \mathrm{O}$ & $\mathrm{C}_{9} \mathrm{H}_{17} \mathrm{~N}_{2}^{+} \cdot \mathrm{C}_{7} \mathrm{H}_{3} \mathrm{~N}_{2} \mathrm{O}_{7}^{-}$ & $\mathrm{C}_{26} \mathrm{H}_{29} \mathrm{~N}_{2}^{+} \cdot \mathrm{C}_{7} \mathrm{H}_{3} \mathrm{~N}_{2} \mathrm{O}_{7}^{-}$ \\
\hline$M_{\mathrm{r}}$ & 481.41 & 398.33 & 380.35 & 596.63 \\
\hline Crystal system, space group & Triclinic, $P \overline{1}$ & Triclinic, $P \overline{1}$ & Monoclinic, $P 2_{1} / n$ & Monoclinic, $P 2_{1} / c$ \\
\hline Temperature (K) & 296 & 100 & 200 & 200 \\
\hline$a, b, c(\AA)$ & $\begin{array}{l}8.5551(1), 10.5000(2) \\
12.7576(3)\end{array}$ & $\begin{array}{l}6.6691(3), 11.3831(4) \\
12.2900(5)\end{array}$ & $\begin{array}{l}6.1537(3), 19.1541(14), \\
14.5527(11)\end{array}$ & $\begin{array}{l}14.5648(3), 12.9374(3), \\
16.1619(3)\end{array}$ \\
\hline$\alpha, \beta, \gamma\left(^{\circ}\right)$ & $\begin{array}{l}106.463(1), 100.913(1), \\
108.272(1)\end{array}$ & $\begin{array}{l}89.727(2), 76.771(2) \\
76.930(2)\end{array}$ & $90,98.343(6), 90$ & $90,103.900(1), 90$ \\
\hline$V\left(\AA^{3}\right)$ & $993.72(3)$ & $883.62(6)$ & $1697.2(2)$ & $2956.22(11)$ \\
\hline$Z$ & 2 & 2 & 4 & 4 \\
\hline Radiation type & Mo $K \alpha$ & Mo $K \alpha$ & Mo $K \alpha$ & Mo $K \alpha$ \\
\hline$\mu\left(\mathrm{mm}^{-1}\right)$ & 0.23 & 0.13 & 0.12 & 0.10 \\
\hline Crystal size $(\mathrm{mm})$ & $0.20 \times 0.20 \times 0.16$ & $0.52 \times 0.13 \times 0.10$ & $0.30 \times 0.13 \times 0.10$ & $0.51 \times 0.26 \times 0.17$ \\
\hline \multicolumn{5}{|l|}{ Data collection } \\
\hline Diffractometer & Bruker Kappa APEXII CCD & $\begin{array}{l}\text { Bruker } S M A R T \text { APEXII } \\
\text { CCD area-detector }\end{array}$ & $\begin{array}{l}\text { Oxford Diffraction Gemini-S } \\
\text { CCD-detector }\end{array}$ & Bruker APEXII CCD \\
\hline Absorption correction & $\begin{array}{l}\text { Multi-scan (SADABS; } \\
\text { Bruker, 2004) }\end{array}$ & $\begin{array}{l}\text { Multi-scan }(S A D A B S \\
\quad \text { Bruker, 2009) }\end{array}$ & $\begin{array}{l}\text { Multi-scan (CrysAlis PRO; } \\
\text { Agilent, 2014) }\end{array}$ & $\begin{array}{l}\text { Multi-scan ( } S A D A B S \text {; } \\
\text { Bruker, 2008) }\end{array}$ \\
\hline$T_{\min }, T_{\max }$ & $0.955,0.964$ & $0.937,0.987$ & $0.920,0.990$ & $0.932,1.000$ \\
\hline $\begin{array}{l}\text { No. of measured, independent } \\
\text { and observed }[I>3 \sigma(I)] \\
\text { reflections }\end{array}$ & $24261,6717,4398$ & $17014,4061,3042$ & $7800,3339,1976$ & $29552,7344,5724$ \\
\hline$R_{\text {int }}$ & 0.030 & 0.030 & 0.034 & 0.015 \\
\hline$(\sin \theta / \lambda)_{\max }\left(\AA^{-1}\right)$ & 0.758 & 0.650 & 0.617 & 0.667 \\
\hline \multicolumn{5}{|l|}{ Refinement } \\
\hline$R$ factors and goodness of fit & $\begin{array}{l}R[F>3 \sigma(F)]=0.044 \\
\quad w R(F)=0.104, S=1.95\end{array}$ & $\begin{array}{l}R[F>3 \sigma(F)]=0.038 \\
\quad w R(F)=0.086, S=1.77\end{array}$ & $\begin{array}{l}R\left[F^{2}>2 \sigma\left(F^{2}\right)\right]=0.046 \\
\quad w R\left(F^{2}\right)=0.095, S=1.33\end{array}$ & $\begin{array}{l}R[F>3 \sigma(F)]=0.054 \\
\quad w R(F)=0.190, S=1.80\end{array}$ \\
\hline No. of reflections & 6717 & 4061 & 3339 & 7344 \\
\hline No. of parameters & 301 & 258 & 268 & 399 \\
\hline No. of restraints & 0 & 0 & 2 & 0 \\
\hline H-atom treatment & $\begin{array}{l}\text { H atoms treated by a } \\
\text { mixture of independent and } \\
\text { constrained refinement }\end{array}$ & $\begin{array}{l}\mathrm{H} \text { atoms treated by a } \\
\text { mixture of independent and } \\
\text { constrained refinement }\end{array}$ & $\begin{array}{l}\text { H atoms treated by a } \\
\text { mixture of independent and } \\
\text { constrained refinement }\end{array}$ & $\begin{array}{l}\mathrm{H} \text { atoms treated by a } \\
\text { mixture of independent } \\
\text { and constrained } \\
\text { refinement }\end{array}$ \\
\hline$\Delta \rho_{\max }, \Delta \rho_{\min }\left(\mathrm{e} \AA^{-3}\right)$ & $0.31,-0.35$ & $0.46,-0.23$ & $0.36,-0.24$ & $0.63,-0.28$ \\
\hline Absolute structure & - & - & - & - \\
\hline Absolute structure parameter & - & - & - & - \\
\hline
\end{tabular}

and recalculated. Tables 1 and 2 contain an overview of those structures, which were successfully redetermined. In the following, these structures are referred to by their CSD refcodes; for the pertinent chemical names, see Table 2.

Notably, JEVNAA turns out not to be a substituted benzoate but a phenolate. NUQVEB though reported as a substituted benzoate turns out to be present in a disordered benzoate and a phenolate form. QIQJAD though reported as a disordered benzoate and a phenolate turns out to be a substituted benzoate. SAFGUD was reported as a substituted benzoate but turns out to be a phenolate. WADXOR was reported as a substituted benzoate that is disordered over two positions but it turns out to be present both in a dominant benzoate as well as in a minor phenolate form. Finally, 
Table 2

Overview of the redetermined structures.

\begin{tabular}{|c|c|}
\hline REFCODE & Chemical name original/corrected if necessary \\
\hline $\mathrm{UJZAK}^{a}$ & Bis(quinolin-8-ol)silver(I) 2-hydroxy-3,5-dinitrobenzoate \\
\hline & $\begin{array}{l}\text { Tetrakis }\left(1 H \text {-imidazole- } N^{3}\right) \text { zinc(II) bis(2-hydroxy-3,5-dinitro- } \\
\text { benzoate / tetrakis }\left(1 H \text {-imidazole- } N^{3}\right) \text { zinc(II) bis(2-carb- } \\
\text { oxy-4,6-dinitrophenolate) }\end{array}$ \\
\hline LUDFUL $^{c}$ & $\begin{array}{l}\text { 1-Aza-8-azoniabicyclo[5.4.0] undec-7-ene 2-hydroxy-3,5-di- } \\
\text { nitrobenzoate / phenazine 2-hydroxy-3,5-dinitrobenzoic } \\
\text { acid }\end{array}$ \\
\hline NUQVEB & $\begin{array}{l}\text { 2-Amino-5-methylpyridinium 2-hydroxy-3,5-dinitrobenzoate) } \\
\text { / 2-amino-5-methylpyridinium 2-hydroxy-3,5-dinitro- } \\
\text { benzoate) }(0.38) \text { / 2-amino-5-methylpyridinium 2-carboxy- } \\
\text { 4,6-dinitrophenolate }(0.62)\end{array}$ \\
\hline QIQJAD & $\begin{array}{l}\text { 3,5-Diamino-6-(2,3-dichlorophenyl)-1,2,4-triazin-2-ium 3,5- } \\
\text { dinitro-2-hydroxybenzoate } N, N \text {-dimethylformamide } \\
\text { solvate / 3,5-dinitro-2-hydroxybenzoate }(0.55) \text { 2-carboxy- } \\
\text { 4,6-dinitrophenolate (0.45) } N, N \text {-dimethylformamide } \\
\text { monosolvate / 3,5-diamino-6-(2,3-dichlorophenyl)-1,2,4- } \\
\text { triazin-2-ium 3,5-dinitro-2-hydroxybenzoate } N, N \text {-di- } \\
\text { methylformamide monosolvate }\end{array}$ \\
\hline SAFGUD $^{f}$ & $\begin{array}{l}\left.\text { Bis(1,10-phenanthroline-5,6-dione-2N, } N^{\prime}\right) \text { silver(I) 2-hydroxy } \\
\text { 3,5-dinitrobenzoate / bis(1,10-phenanthroline-5,6-dione- } \\
\left.2 N, N^{\prime}\right) \text { silver(I) 2-carboxy-4,6-dinitrophenolate }\end{array}$ \\
\hline SEDKET $^{g}$ & $\begin{array}{l}\text { 3,5-Dimethylpyrazolium 2-carboxy-4,6-dinitr } \\
\text { 3,5-dimethylpyrazolium 2-hydroxy-3,5-dini }\end{array}$ \\
\hline TIYZI $M^{h}$ & $\begin{array}{l}\text { 3-(1H-Imidazol-1-yl)propanaminium 2-carboxy-4,6-dinitro- } \\
\text { phenolate }\end{array}$ \\
\hline TUJPEV $^{i}$ & $\begin{array}{l}\text { 4-[(5-methylisoxazol-3-yl)aminosulfonyl]anilinium 3,5-di- } \\
\text { nitrosalicylate }\end{array}$ \\
\hline $\mathrm{VABZIJ}^{j}$ & $\begin{array}{l}\text { 2-Isopropyl-6-methyl-4-oxo-3,4-dihydropyrimidin-1-ium } \\
\text { 2-carboxy-4,6-dinitrophenolatemonohydrate }\end{array}$ \\
\hline WADXOR & $\begin{array}{l}\text { 1-Aza-8-azoniabicyclo[5.4.0]undec-7-ene 2-hydroxy-3,5-di- } \\
\text { nitrobenzoate / 2,3,4,6,7,8,9,10-octahydropyrimido[1,2-a] } \\
\text { azepin-1-ium 2-hydroxy-3,5-dinitrobenzoate }(0.73) / \\
\text { 2,3,4,6,7,8,9,10-octahydropyrimido[1,2-a]azepin-1-ium } \\
\text { 2-carboxy-4,6-2-carboxy-4,6-dinitrophenolate }(0.37)\end{array}$ \\
\hline YAXPO & $\begin{array}{l}\text { 4-(Diphenylmethyl)-1-(3-phenylprop-2-en-1-yl)piperazin-1- } \\
\text { ium 2-carboxy-4,6-dinitrophenolate }\end{array}$ \\
\hline
\end{tabular}

Notes: (a) Zhang \& Jian (2009); (b) Huang et al. (2007); (c) Senthil Kumar et al. (2002); (d) Hemamalini \& Fun (2010a); (e) Sridhar et al. (2013); ( $f$ ) Wang et al. (2012); ( $g$ ) Wei et al. (2012); (h) Yamuna et al. (2014); (i) Malathy et al. (2015); (j) Hemamalini \& Fun (2010b); (k) Smith \& Lynch (2016); (l) Dayananda et al. (2012).

SEDKET was originally determined as a substituted phenolate but it turns out to be a benzoate.

Some of the retrieved structures were difficult or impossible to recalculate with sufficient accuracy: HILPOI (trimethoprimium 3,5-dinitrosalicylate; Subashini et al., 2007) because of an abnormally low proportion of observed reflections (moreover the bridging hydrogen $\mathrm{H} 6 a$ is situated out of the plane between the carboxylate and hydroxy oxygen atoms, which seems to indicate an error) and VUZNEK (3,4-diaminopyridinium 2-carboxy-4,6-dinitrophenolate; Hemamalini \& Fun, 2010b) because of the disorder present in the structure.

\section{Refinement of the title structures}

For each structure, two methods have been applied for the refinement of the hydrogen atoms involved in hydrogen bonding. In Method 1, the positions of the bridging hydrogens as well as those of the hydroxy, primary and secondary amine and ammonium hydrogen atoms were fixed after their localization in the difference electron-density maps while their displacement parameters were refined. In Method 2, the positional parameters of the latter hydrogen atoms were refined while their displacement parameters were constrained in the usual manner: $U_{\text {iso }}(\mathrm{H})=1.2 U_{\text {eq }}\left(\mathrm{N}_{\text {amine }}\right)$ or $U_{\text {iso }}(\mathrm{H})=$ $1.5 U_{\text {eq }}\left(\mathrm{O}_{\text {hydroxy }}\right)$ or $U_{\text {iso }}(\mathrm{H})=1.5 U_{\text {eq }}\left(\mathrm{N}_{\text {ammonium }}\right)$.

The appropriate sections of the difference electron-density maps of the title structures (see supplementary Fig. S1) show regions with the hydroxy, amine and ammonium hydrogen atoms. These sections comprise the maps that were obtained after the refinement of the models without the pertinent hydrogen atoms as well as the maps that were calculated by either refinement method. It can be seen from the supplementary Fig. S1 that one of the reasons that hinders the correct localization of the hydrogen atoms involved in the hydrogen bonds is an apparent non-spherical electron density of the donor and acceptor atoms. Thus, hydrogen-atom localization by X-ray diffraction is hindered not only by its weak scattering power, but also by the polarization of its electron density resulting from the proximity of the acceptor and by the asphericity of the electron density of the donor and acceptor atoms. Therefore, refinement Method 1 was given preference. The hydrogen bonds in the title structures are listed in Table 3, which shows that there might be quite a large difference between the results with the fixed and the refined positional parameters of such hydrogen atoms. In the following, a detailed description of the refinement of the recalculated structures is given:

DUJZAK (Zhang \& Jian, 2009): $\mathrm{C}-\mathrm{H}_{\text {aryl }}$ were constrained to be equal to $0.93 \AA$ while $U_{\text {iso }}\left(\mathrm{H}_{\text {aryl }}\right)=1.2 U_{\text {eq }}\left(\mathrm{C}_{\text {aryl }}\right)$. The position of the bridging hydrogen $\mathrm{H} 3 b$ as well as those of the hydroxy hydrogen atoms $\mathrm{H} 1 a a$ and $\mathrm{H} 2 a a$ were located in a difference electron-density map. Their positional parameters were fixed during the refinement while their isotropic displacement parameters were refined.

JEVNAA (Huang et al., 2007): $\mathrm{C}-\mathrm{H}_{\text {aryl }}$ were constrained to be equal to $0.93 \AA$ while $U_{\text {iso }}\left(\mathrm{H}_{\text {aryl }}\right)=1.2 U_{\text {eq }}\left(\mathrm{C}_{\text {aryl }}\right)$. The position of the bridging hydrogen $\mathrm{H} 1 a$ as well as those of the secondary amine hydrogen atoms $\mathrm{H} 2 a$ and $\mathrm{H} 4 a$ were located in the difference electron-density map. Their positional parameters were fixed during the refinement while their isotropic displacement parameters were refined.

LUDFUL (Senthil Kumar et al., 2002): $\mathrm{C}-\mathrm{H}_{\text {aryl }}$ were constrained to be equal to $0.93 \AA$ while $U_{\text {iso }}\left(\mathrm{H}_{\text {aryl }}\right)=$ $1.2 U_{\text {eq }}\left(\mathrm{C}_{\text {aryl }}\right)$. The position of the bridging hydrogen $\mathrm{H} 3 a$ as well as that of the hydroxy hydrogen atom $\mathrm{H} 1 a$ were located in a difference electron-density map. Their positional parameters were fixed during the refinement while their isotropic displacement parameters were refined.

NUQVEB (Hemamalini \& Fun, 2010a): The subroutine TwinRotMax of PLATON (Spek, 2009) indicated non-merohedral twinning: $h 2=-h 1 ; k 2=-k 1 ; l 2=-0.488 h 1-0.153 k 1$ $+l 1$. The refinement was carried out on the non-overlapped reflections only. The refined value of the second domain fraction converged to the value -0.0006 (4). Therefore the value of the second domain fraction was set to 0 and was not refined further. $\mathrm{C}-\mathrm{H}_{\text {aryl }}$ and $\mathrm{C}-\mathrm{H}_{\text {methyl }}$ were constrained to be equal to 0.95 and $0.98 \AA$, respectively. $U_{\text {iso }}\left(\mathrm{H}_{\text {aryl }}\right)=$ 
Table 3

Hydrogen bonds $\left(\AA,^{\circ}\right)$ in the redetermined structures.

The upper entries for each hydrogen bond refer to refinement Method 1: fixed hydrogen-atom positions, which were obtained from the difference electrondensity maps, and refined displacement parameters. The lower entries refer to refinement Method 2: refined hydrogen-atom positions and constrained displacement parameters.

\begin{tabular}{|c|c|c|c|c|}
\hline$D-\mathrm{H} \cdots A$ & $D-\mathrm{H}$ & $\mathrm{H} \cdots A$ & $D \cdots A$ & $D-\mathrm{H} \cdots A$ \\
\hline \multicolumn{5}{|l|}{ DUJZAK } \\
\hline \multirow{2}{*}{$\mathrm{O} 1-\mathrm{H} 1 a a \cdots \mathrm{O} 8$} & $0.759(2)$ & $1.859(2)$ & $2.606(3)$ & $167.96(14)$ \\
\hline & $0.97(4)$ & $1.64(4)$ & $2.603(3)$ & $175(3)$ \\
\hline \multirow[t]{2}{*}{$\mathrm{O} 2-\mathrm{H} 2 a a \cdots \mathrm{O} 9$} & $0.922(2)$ & $1.727(2)$ & $2.631(3)$ & 166.48 \\
\hline & $0.75(4)$ & $1.90(4)$ & $2.636(3)$ & $165(4)$ \\
\hline \multirow[t]{2}{*}{$\mathrm{O} 3-\mathrm{H} 3 b \cdots \mathrm{O} 9$} & $1.040(2)$ & $1.495(2)$ & $2.481(3)$ & $155.88(12)$ \\
\hline & $1.11(4)$ & $1.41(4)$ & $2.480(3)$ & $160(3)$ \\
\hline
\end{tabular}

JEVNAA

$\mathrm{O} 2-\mathrm{H} 1 a \cdots \mathrm{O} 1$

$\mathrm{N} 2-\mathrm{H} 2 a \cdots \mathrm{O} 3$

$\mathrm{N} 4-\mathrm{H} 4 a \cdots \mathrm{O}^{\mathrm{i}}$

$\begin{array}{lll}1.039(2) & 1.496(2) & 2.498(2) \\ 0.89(2) & 1.65(3) & 2.503(2) \\ 0.967(2) & 1.890(2) & 2.838(3) \\ 0.84(2) & 2.02(2) & 2.845(3) \\ 0.943(2) & 1.924(1) & 2.784(2) \\ 0.86(2) & 1.95(2) & 2.792(2)\end{array}$

$160.4(1)$

$160(2)$

$165.9(1)$

$169(2)$

$150.6(1)$

$165(2)$

LUDFUL

$\mathrm{O} 3-\mathrm{H} 3 a \cdots \mathrm{O} 2$

$\mathrm{O} 1-\mathrm{H} 1 a \cdots \mathrm{N} 3$

$\begin{array}{lll}1.059(1) & 1.530(1) & 2.513(2) \\ 1.06(2) & 1.51(2) & 2.516(2) \\ 1.163(1) & 1.416(1) & 2.552(2) \\ 1.14(2) & 1.44(2) & 2.552(2)\end{array}$

$151.7(1)$

$156(2)$

$163.2(1)$

166 (2)

NUQVEB

$\mathrm{O} 7-\mathrm{H} 107 \cdots \mathrm{O} 1$

$\mathrm{O} 1-\mathrm{H} 1 \mathrm{o} 1 \cdots \mathrm{O} 7$

$\mathrm{N} 2-\mathrm{H} 2 a \cdots \mathrm{O} 7^{\mathrm{ii}}$

$\mathrm{N} 2-\mathrm{H} 2 b \cdots \mathrm{O} 1^{\mathrm{iii}}$

$\mathrm{N} 2-\mathrm{H} 2 b \cdots \mathrm{O} 2^{\mathrm{iii}}$

$\mathrm{N} 1-\mathrm{H} 1 \cdots \mathrm{O} 6^{\mathrm{ii}}$

$\begin{array}{llll}0.919(1) & 1.531(1) & 2.4202(12) & 161.55(6) \\ 1.14(2) & 1.31(2) & 2.4178(12) & 163(2) \\ 0.931(1) & 1.513(1) & 2.4202(12) & 163.52(6) \\ 1.31(2) & 1.14(2) & 2.4178(12) & 163(2) \\ 0.892(1) & 2.079(1) & 2.9655(14) & 172.84(6) \\ 0.87(1) & 2.095(14) & 2.9674(14) & 176.5(12) \\ 0.846(1) & 2.165(1) & 2.8526(14) & 138.40(6) \\ 0.88(2) & 2.146(14) & 2.852(1) & 137.3(11) \\ 0.846(1) & 2.413(1) & 3.1741(14) & 150.02(6) \\ 0.88(2) & 2.384(14) & 3.1736(15) & 150.3(11) \\ 0.898(1) & 1.783(1) & 2.6781(13) & 174.83(6) \\ 0.90(1) & 1.784(14) & 2.6773(14) & 173.3(13)\end{array}$

QIQJAD

$\mathrm{N} 3-\mathrm{H} 3 n \cdots \mathrm{O} 2$

$\mathrm{N} 3-\mathrm{H} 4 n \cdots \mathrm{O}^{\mathrm{iv}}$

$\mathrm{N} 2-\mathrm{H} 2 n \cdots \mathrm{O} 1$

$\mathrm{N} 5-\mathrm{H} 5 n \cdots \mathrm{N} 4^{\mathrm{v}}$

$\mathrm{N} 5-\mathrm{H} 6 n \cdots \mathrm{O} 8^{\mathrm{vi}}$

$\mathrm{O} 3-\mathrm{H} 3 o \cdots \mathrm{O} 1$

$\begin{array}{llll}0.862(2) & 1.994(2) & 2.854(2) & 174.8(1) \\ 0.81(3) & 2.05(3) & 2.854(3) & 175(3) \\ 0.863(2) & 2.059(1) & 2.921(2) & 176.9(1) \\ 0.85(2) & 2.07(2) & 2.920(2) & 173(3) \\ 0.897(2) & 1.831(2) & 2.728(2) & 177.5(1) \\ 0.81(3) & 1.93(3) & 2.731(2) & 171(2) \\ 0.866(1) & 2.141(1) & 2.9992(19) & 171.1(1) \\ 0.84(2) & 2.17(2) & 2.999(2) & 171(2) \\ 0.863(2) & 2.041(2) & 2.760(2) & 140.2(1) \\ 0.78(2) & 2.12(3) & 2.764(2) & 141(2) \\ 0.926(1) & 1.562(1) & 2.4572(18) & 161.3(1) \\ 0.99(3) & 1.49(3) & 2.4569(19) & 164(3)\end{array}$

SAFGUD

$\mathrm{O} 8-\mathrm{H} 7 \cdots \mathrm{O} 7$

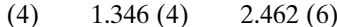
$\begin{array}{lll}1.05(7) & 1.57(7) \quad 2.452(7)\end{array}$

$159.6(3)$ $138(6)$

SEDKET

$\begin{array}{lllll}\mathrm{O} 1-\mathrm{H} 2 a \cdots \mathrm{O} 2 & 1.22(5) & 1.34(5) & 2.476(3) & 149(5) \\ & 1.27(3) & 1.29(3) & 2.477(3) & 151(3) \\ \mathrm{O} 2-\mathrm{H} 2 a \cdots \mathrm{O} 1 & 1.34(5) & 1.22(5) & 2.476(3) & 149(5) \\ & 1.29(3) & 1.27(3) & 2.477(3) & 151(3) \\ \mathrm{N} 1-\mathrm{H} 1 \cdots \mathrm{O} 1^{\mathrm{vii}} & 1.11(5) & 1.92(5) & 2.799(4) & 133(3) \\ & 0.99(4) & 2.00(3) & 2.804(4) & 137(3) \\ \mathrm{N} 1-\mathrm{H} 1 \cdots \mathrm{O} 7^{\mathrm{vii}} & 1.11(5) & 1.94(5) & 2.850(4) & 137(3) \\ & 0.99(4) & 2.03(3) & 2.855(4) & 140(3) \\ \mathrm{N} 2-\mathrm{H} 2 \cdots \mathrm{O} 3 & 0.96(3) & 1.77(3) & 2.685(4) & 158(3) \\ & 0.99(3) & 1.75(3) & 2.684(4) & 157(3)\end{array}$

Table 3 (continued)

\begin{tabular}{lllll}
\hline$D-\mathrm{H} \cdots A$ & $D-\mathrm{H}$ & $\mathrm{H} \cdots A$ & $D \cdots A$ & $D-\mathrm{H} \cdots A$ \\
\hline
\end{tabular}

TIYZIM

$\begin{array}{lllll}\mathrm{O} 2 b-\mathrm{H} 2 b \cdots \mathrm{O} 1 b & 0.982(1) & 1.516(1) & 2.4473(16) & 156.3(1) \\ & 1.02(2) & 1.48(2) & 2.4476(16) & 156(2) \\ \mathrm{N} 3 a-\mathrm{H} 3 a a \cdots \mathrm{N} 1 a a^{\text {viii }} & 0.904(1) & 1.932(1) & 2.797(2) & 159.6(1) \\ & 0.91 & 1.92 & 2.797(2) & 162 \\ \mathrm{~N} 3 a-\mathrm{H} 3 a b \cdots \mathrm{O} 2 b^{\text {ix }} & 0.901(1) & 2.565(1) & 3.1297(17) & 121.4(1) \\ & 0.91 & 2.58 & 3.1298(17) & 120 \\ \mathrm{~N} 3 a-\mathrm{H} 3 a b \cdots \mathrm{O} 2 b^{\text {ix }} & 0.901(1) & 2.565(1) & 3.1297(17) & 121.4(1) \\ & 0.91 & 2.58 & 3.1297(18) & 120 \\ \mathrm{~N} 3 a-\mathrm{H} 3 a b \cdots \mathrm{O} 3 b^{\text {ix }} & 0.901(1) & 2.072(1) & 2.9537(17) & 165.8(1) \\ & 0.91 & 2.06 & 2.9542(17) & 165 \\ \mathrm{~N} 3 a-\mathrm{H} 3 a c \cdots \mathrm{O} 1 b^{x} & 0.893(1) & 2.061(1) & 2.815(2) & 141.5(1) \\ & 0.91 & 2.03 & 2.815(2) & 144 \\ \mathrm{~N} 3 a-\mathrm{H} 3 a c \cdots \mathrm{O} 7 b^{x} & 0.893(1) & 2.484(1) & 2.9712(19) & 114.7(1) \\ & 0.91 & 2.46 & 2.9706(19) & 116\end{array}$

TUJPEV

O6-H6a $\cdots$ O5

$\mathrm{N} 1-\mathrm{H} 1 a \cdots \mathrm{O} 6^{\mathrm{xi}}$

$\mathrm{N} 1-\mathrm{H} 1 b \cdots \mathrm{N} 3^{\mathrm{v}}$

$\mathrm{N} 1-\mathrm{H} 1 c \cdots \mathrm{O} 4^{\mathrm{v}}$

$\mathrm{N} 2-\mathrm{H} 2 a \cdots \mathrm{O} 5$

$\begin{array}{llll}1.184(1) & 1.295(1) & 2.4268(16) & 156.58(6) \\ 1.24(2) & 1.21(2) & 2.4280(17) & 165.3(14) \\ 1.002(1) & 2.068(1) & 3.0655(17) & 173.55(7) \\ 0.89 & 2.24 & 3.0694(17) & 155 \\ 0.793(1) & 2.292(1) & 3.0393(15) & 157.3(1) \\ 0.89 & 2.20 & 3.0382(15) & 157 \\ 0.832(2) & 1.831(1) & 2.663(2) & 177.1(1) \\ 0.89 & 1.77 & 2.660(2) & 175 \\ 0.970(1) & 1.844(1) & 2.7852(15) & 162.64(9) \\ 0.827(17) & 1.986(16) & 2.7900(16) & 164.0(18)\end{array}$

VABZIJ

$\begin{array}{lllll}\mathrm{N} 3-\mathrm{H} 1 n 3 \cdots \mathrm{O}^{x} & 0.973(1) & 1.754(1) & 2.7182(14) & 170.48(8) \\ & 0.91(1) & 1.823(14) & 2.7214(14) & 170.8(15) \\ \mathrm{N} 4-\mathrm{H} 1 n 4 \cdots \mathrm{O} 1 w & 0.909(1) & 1.840(1) & 2.7348(15) & 167.76(8) \\ & 0.91(2) & 1.833(15) & 2.7323(16) & 172.0(15) \\ \mathrm{O} 1 w-\mathrm{H} 2 w 1 \cdots \mathrm{O} 1^{\mathrm{xii}} & 0.917(1) & 1.890(1) & 2.7886(14) & 166.21(6) \\ & 0.82(2) & 1.995(19) & 2.7906(15) & 162.7(16) \\ \mathrm{O} 1 w-\mathrm{H} 1 w 1-\mathrm{O}^{\mathrm{iii}} & 0.915(1) & 2.040(1) & 2.9352(14) & 165.84(7) \\ & 0.89(2) & 2.064(18) & 2.9357(15) & 168.0(17) \\ \mathrm{O} 7-\mathrm{H} 7 \cdots \mathrm{O} 1 & 1.019(1) & 1.433(1) & 2.4340(13) & 165.94(7) \\ & 0.96(2) & 1.505(16) & 2.4358(13) & 162.0(16)\end{array}$

WADXOR

$\mathrm{N} 8 a-\mathrm{H} 8 a \cdots \mathrm{O} 11 b \quad 0.960(2) \quad 1.933(2) \quad 2.864(2) \quad 162.83(11)$ $\begin{array}{llll}0.91(2) & 1.96(2) & 2.869(2) & 174.1(17)\end{array}$

$\begin{array}{lllll}\mathrm{O} 11 b-\mathrm{H} 21 b \cdots \mathrm{O} 21 b & 1.145(2) & 1.303(6) & 2.433(6) & 167.3(3)\end{array}$

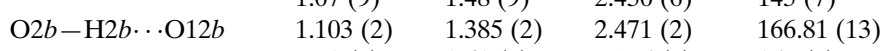
$0.91(3) \quad 1.61(3) \quad 2.475(3) \quad 159(3)$

YAXPOE

$\mathrm{N} 1-\mathrm{H} 71 \cdots \mathrm{O} 1^{\text {iv }} \quad 0.945(1) \quad 1.954(1) \quad 2.813(2) \quad 150.01(8)$

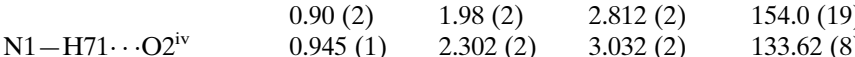

$\begin{array}{lllll} & 0.90(2) & 2.36(2) & 3.034(2) & 131.8(17) \\ \mathrm{O} 7-\mathrm{H} 7 \ldots \mathrm{O} 1 & 0.924(2) & 1.668(1) & 2.505(2) & 148.96(9)\end{array}$

$\begin{array}{llll}0.92(3) & 1.71(3) & 2.504(2) & 142(2)\end{array}$

Symmetry codes: (i) $-x+\frac{3}{2},-y+\frac{1}{2},-z+1$; (ii) $-x+1,-y+1,-z$; (iii) $-x+1,-y,-z$; (iv) $-x+1,-y+1,-z+1$; (v) $-x+1,-y+2,-z+1$; (vi) $x-1, y+1, z$; (vii) $-x+1, y+\frac{1}{2}$, $-z+1$; (viii) $-x,-y,-z$; (ix) $x+1, y, z ;(x)-x,-y+1,-z+1 ;$ (xi) $-x,-y+2,-z+1$; (xii) $x, y+1, z$.

$1.2 U_{\text {eq }}\left(\mathrm{C}_{\text {aryl }}\right)$ and $U_{\text {iso }}\left(\mathrm{H}_{\text {methyl }}\right)=1.5 U_{\text {eq }}\left(\mathrm{C}_{\text {methyl }}\right)$. The positions of the disordered bridging hydrogens $\mathrm{H} 101$ and $\mathrm{H} 107$ as well as those of the primary $(\mathrm{H} 2 a, \mathrm{H} 2 b)$ and the secondary amine hydrogen atoms ( $\mathrm{H} 1 a)$ were located in a difference electrondensity map. Their positional parameters were fixed during the refinement while their isotropic displacement parameters were refined; in the case of the bridging hydrogens $\mathrm{H} 1 o 1$ and 
$\mathrm{H} 1 \mathrm{o}$, their isotropic displacement parameters were refined to be equal while their occupational parameters were refined under the condition that their sum was equal to 1 .

QIQJAD (Sridhar et al., 2013): The subroutine TwinRotMax of PLATON (Spek, 2009) indicated non-merohedral twinning: $h 2=-1.018 h 1+0.054 k 1 ; k 2=-0.673 h 1+1.018 k 1$; $l 2=-0.039 h 1+0.116 k 1-l 1$. The refined value of the second domain fraction converged to the value 0.028 (13). Therefore the value of the second domain fraction was set to 0 and was not refined further. $\mathrm{C}-\mathrm{H} s p^{2}$ and $\mathrm{C}-\mathrm{H}_{\text {methyl }}$ were constrained to equal to 0.93 and $0.96 \AA$, respectively. $U_{\text {iso }}\left(\mathrm{H} s p^{2}\right)=$ $1.2 U_{\text {eq }}\left(\mathrm{Csp}^{2}\right)$ and $U_{\text {iso }}\left(\mathrm{H}_{\text {methyl }}\right)=1.5 U_{\text {eq }}\left(\mathrm{C}_{\text {methyl }}\right)$. The positions of the bridging hydrogen $\mathrm{H} 3 o$ and those of the primary $(\mathrm{H} 3 n$, $\mathrm{H} 4 n, \mathrm{H} 5 n, \mathrm{H} 6 n)$ as well as of the secondary $(\mathrm{H} 2 n)$ amine hydrogen atoms were located in a difference electron-density map. Their positional parameters were fixed during the refinement while their isotropic displacement parameters were refined.

SAFGUD (Wang et al., 2012): $\mathrm{C}-\mathrm{H}_{\text {aryl }}$ were constrained to be equal to $0.93 \AA$ while $U_{\text {iso }}\left(\mathrm{H}_{\text {aryl }}\right)=1.2 U_{\text {eq }}\left(\mathrm{C}_{\text {aryl }}\right)$. The bridging hydrogen $\mathrm{H} 7$ was located in a difference electrondensity map and its position was fixed while its isotropic displacement parameter $U_{\text {iso }}(\mathrm{H} 7)$ was refined.

SEDKET (Wei et al., 2012): The non-centrosymmetric structure is composed of the light atoms only (the heaviest atom is $\mathrm{O}$ ) and the data collection was carried out with Mo $K \alpha$ radiation. The article by Wei et al. (2012) does not indicate whether the Friedel pairs were merged and nor does it contain the value of the Flack parameter. The Flack parameter was set to 0.5 without being refined in the present model. $\mathrm{C}-\mathrm{H}_{\text {aryl }}$ and $\mathrm{C}-\mathrm{H}_{\text {methyl }}$ were constrained to be equal to 0.93 and $0.96 \AA$, respectively. $U_{\text {iso }}\left(\mathrm{H}_{\text {aryl }}\right)=1.2 U_{\text {eq }}\left(\mathrm{C}_{\text {aryl }}\right)$ and $U_{\text {iso }}\left(\mathrm{H}_{\text {methyl }}\right)=$ $1.5 U_{\text {eq }}\left(\mathrm{C}_{\text {methyl }}\right)$. The position of the bridging hydrogen $\mathrm{H} 2 a$ as well as those of the secondary amine hydrogen atoms $\mathrm{H} 1$ and $\mathrm{H} 2$ were located in a difference electron-density map. Their positional parameters were fixed during the refinement while their isotropic displacement parameters were refined.

TIYZIM (Yamuna et al. (2014): $\mathrm{C}-\mathrm{H}_{\text {aryl }}$ and $\mathrm{C}-\mathrm{H}_{\text {methylene }}$ were constrained to be equal to 0.95 and $0.99 \AA$, respectively. $U_{\text {iso }}\left(\mathrm{H}_{\text {aryl }}\right)=1.2 U_{\text {eq }}\left(\mathrm{C}_{\text {aryl }}\right)$ and $U_{\text {iso }}\left(\mathrm{H}_{\text {methylene }}\right)=$ $1.5 U_{\text {eq }}\left(\mathrm{C}_{\text {methylene }}\right)$. The position of the bridging hydrogen $\mathrm{H} 2 b$ as well as those of the ammonium hydrogen atoms ( $\mathrm{H} 3 a a$, $\mathrm{H} 3 a b, \mathrm{H} 3 a c$ ) were found in a difference electron-density map. Their positional parameters were fixed during the refinement while their isotropic displacement parameters were refined; in the case of the ammonium hydrogen atoms ( $\mathrm{H} 3 a b, \mathrm{H} 3 a c$,), their displacement parameters were constrained to be equal to that of H3aa.

TUJPEV (Malathy et al., 2015): $\mathrm{C}-\mathrm{H}_{\text {aryl }}$ were constrained to be equal to $0.93 \AA$ while $U_{\text {iso }}\left(\mathrm{H}_{\text {aryl }}\right)=1.2 U_{\text {eq }}\left(\mathrm{C}_{\text {aryl }}\right) . \mathrm{C}-$ $\mathrm{H}_{\text {methyl }}$ were constrained to be equal to $0.96 \AA$ while $U_{\text {iso }}\left(\mathrm{H}_{\text {methyl }}\right)=1.5 U_{\text {eq }}\left(\mathrm{C}_{\text {methyl }}\right)$. The position of the bridging hydrogen $\mathrm{H} 6 a$ as well as those of the secondary amine group $\mathrm{H} 2 a$ and of the ammonium hydrogen atoms $\mathrm{H} 1 a, \mathrm{H} 1 b$ and $\mathrm{H} 1 c$ were found in a difference-electron map. Their positional parameters were fixed during the refinement while their isotropic displacement parameters were refined; in the case of the ammonium hydrogen atoms $(\mathrm{H} 1 b, \mathrm{H} 1 c)$, their displacement parameters were constrained to be equal to that of $\mathrm{H} 1 a$.

VABZIJ (Hemamalini \& Fun, 2010c): $\mathrm{C}-\mathrm{H}_{\text {aryl }}, \mathrm{C}-\mathrm{H}_{\text {methyl }}$, $\mathrm{C}-\mathrm{H}_{\text {methine }}$ were constrained to be equal to $0.93,0.96$ and $0.98 \AA$, respectively. $U_{\text {iso }}\left(\mathrm{H}_{\text {aryl }}\right)=1.2 U_{\text {eq }}\left(\mathrm{C}_{\text {aryl }}\right), U_{\text {iso }}\left(\mathrm{H}_{\text {methine }}\right)$ $=1.2 U_{\text {eq }}\left(\mathrm{C}_{\text {methine }}\right), U_{\text {iso }}\left(\mathrm{H}_{\text {methyl }}\right)=1.5 U_{\text {eq }}\left(\mathrm{C}_{\text {methyl }}\right)$. The position of the bridging hydrogen $\mathrm{H} 7$ as well as those of the secondary amine hydrogen atom $\mathrm{H} 1 n 4$ and of the water hydrogen atoms $\mathrm{H} 1 w 1$ and $\mathrm{H} 1 w 2$ were located in a difference electron-density map. Their positional parameters were fixed during the refinement while their displacement parameters were refined.

WADXOR (Smith \& Lynch, 2016): The non-centrosymmetric structure is composed of light atoms only (the heaviest atoms present in the structure are oxygens) and the data collection was carried out with Mo $K \alpha$ radiation. The original article reported the refined Flack parameter to be equal to -0.1 (13); however, the refinement using JANA2006 (Petř́ícek et al., 2014) did not converge and therefore the Flack parameter was set to 0.5 without being refined. $\mathrm{C}-\mathrm{H}_{\text {aryl }}$ and $\mathrm{C}-$ $\mathrm{H}_{\text {methylene }}$ were constrained to be equal to 0.95 and $0.99 \AA$, respectively, except for the distances between the methylene atom $\mathrm{C} 11$ and the attached hydrogen atoms H12a and H13a, which were restrained to 0.99 (1) $\AA$ (Müller, 2009). [The reason for the different treatment of the latter methylene group was its vicinity to the disordered methylene groups centered on $\mathrm{C} 10$ and $\mathrm{C} 12 a$.] $U_{\text {iso }}\left(\mathrm{H}_{\text {aryl }}\right)=1.2 U_{\text {eq }}\left(\mathrm{C}_{\text {aryl }}\right)$ and $U_{\text {iso }}\left(\mathrm{H}_{\text {methylene }}\right)=1.2 U_{\text {eq }}\left(\mathrm{C}_{\text {methylene }}\right)$. There were two types of occupational disorder present in the structure. The first one was related to the fragments with the methylene carbon atoms $\mathrm{C} 9 a, \mathrm{C} 10 a$ and the attached respective pairs of hydrogen atoms H91a, H92a and $\mathrm{H} 10 a, \mathrm{H} 11 a$, as well as to $\mathrm{C} 13 a$ and $\mathrm{C} 12 a$ with the attached respective pairs of hydrogen atoms $\mathrm{H} 16 a, \mathrm{H} 17 a$ and H14a, H15a. The occupation parameter of C13 was refined while those of the related atoms were either set equal to that of $\mathrm{C} 13$ (i.e. $\mathrm{C} 12 a$ and attached hydrogen atoms) or its complement to 1 (C9a and $\mathrm{C} 10 a$ and attached hydrogen atoms). The displacement parameters of the disordered pairs of atoms C9a and C13a as well as C10 $a$ and $\mathrm{C} 12 a$ were set to be equal, i.e. that of $\mathrm{C} 13 a$ equalled that of $\mathrm{C} 9 a$ while that of $\mathrm{C} 10 a$ equalled that of $\mathrm{C} 12 a$. The second type of occupational disorder referred to the fragments $\mathrm{C} 2 b-\mathrm{H} 61 b$, $\mathrm{C} 2 b-\mathrm{O} 2 b-\mathrm{H} 2 b$ and $\mathrm{C} 6 b-\mathrm{H} 6 b, \mathrm{C} 6 b-\mathrm{O} 21 b-\mathrm{H} 21 b$. This means that the occupation parameters of $\mathrm{H} 61 b, \mathrm{H} 21 b$ were set equal to the refined occupational parameter of $\mathrm{O} 21 b$ while being complements to 1 for $\mathrm{H} 6 b, \mathrm{O} 2 b, \mathrm{H} 2 b$. The positions of the bridging hydrogens $\mathrm{H} 2 b$ and $\mathrm{H} 21 b$ as well as that of the primary amine hydrogen atom $\mathrm{H} 8 a$ were located in a difference electron-density map. Their positional parameters were fixed during the refinement while their isotropic displacement parameters were refined; in the case of bridging hydrogens $\mathrm{H} 2 b$ and $\mathrm{H} 21 b$, their isotropic displacement parameters were constrained to be equal.

YAXPOE (Dayananda et al., 2012): $\mathrm{C}-\mathrm{H}_{\text {aryl }}$ and $\mathrm{C}-$ $\mathrm{H}_{\text {methylene }}$ were constrained to equal to 0.95 and $0.99 \AA$, respectively. $U_{\text {iso }}\left(\mathrm{H}_{\text {aryl }}\right)=1.2 U_{\text {eq }}\left(\mathrm{C}_{\text {aryl }}\right)$ and $U_{\text {iso }}\left(\mathrm{H}_{\text {methylene }}\right)=$ $1.5 U_{\text {eq }}\left(\mathrm{C}_{\text {methylene }}\right)$. The bridging hydrogen $\mathrm{H} 7$ was located in a difference electron-density map. Its positional parameters 
were fixed while $U_{\text {iso }}(\mathrm{H} 7 a)$ was refined. A high instability factor $\Delta$ in the weighting scheme (0.0064) was applied in order to avoid a large number of reflections with $\left(I_{\text {obs }}-I_{\text {calc }}\right) / \sigma(w)>10$ where $\sigma(w)=\left[\sigma^{2}(I)+\Delta I^{2}\right]^{-1 / 2}$. [This condition generates $\mathrm{A}$ alerts for $\Delta=0.0004$, which has been used in other refinements of the title structure, when running checkCIF (Spek, 2009).] The residual electron-density map contains peaks which are difficult to interpret (see supplementary Fig. S1).

\section{Discussion of the interdependence of bond lengths and angles}

For this discussion, the definition of the various bonds and angles in the moieties of I-IV (shown in the scheme), are illustrated in Figs. $1 a$ and $1 b$, respectively. As already pointed out, the dependence $D 2$ on $D 4$ and $D 1$ on $D 3$ (Fig. 2) has shown that a large number of structures are biased by incorrectly applied constraints or restraints on the bridging hydrogen. However, a dubious or incorrect localization of the

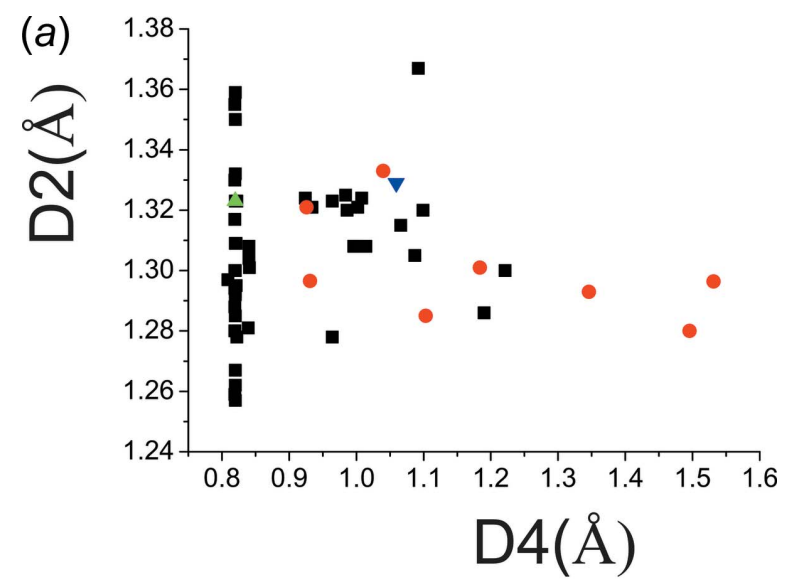

(b)

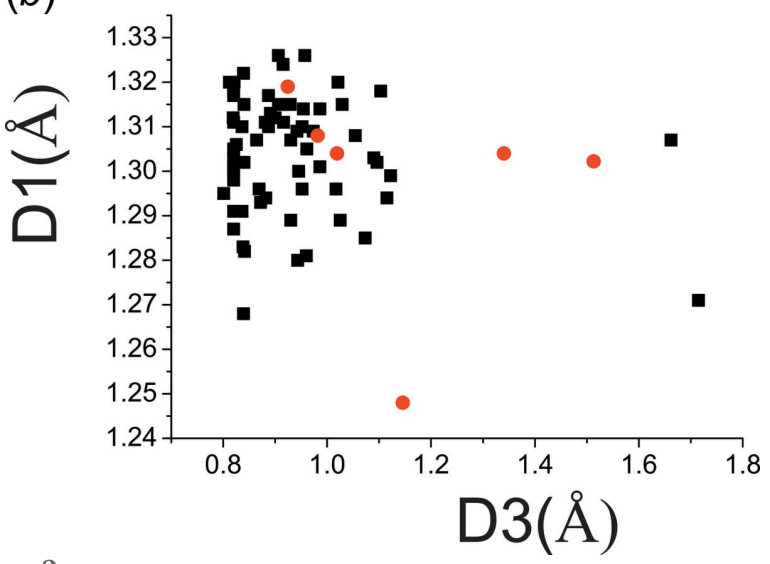

Figure 2

The dependence of bond distances: (a) $D 2$ on $D 4$ for structures that were originally determined as 2-hydroxy-3,5-dinitrobenzoate (II), or as containing 2-hydroxy-3,5-dinitrobenzoic acid (I); (b) D1 on $D 3$ for the structures that were determined as 2-carboxy-4,6-dinitrophenolate (III). Colour code for symbols: black squares are the data retrieved from the CSD; red circles are the corrected title structures; green and blue triangles are the original and the corrected structure of LUDFUL, which contains a molecule of 2-hydroxy-3,5-dinitrobenzoic acid (I).
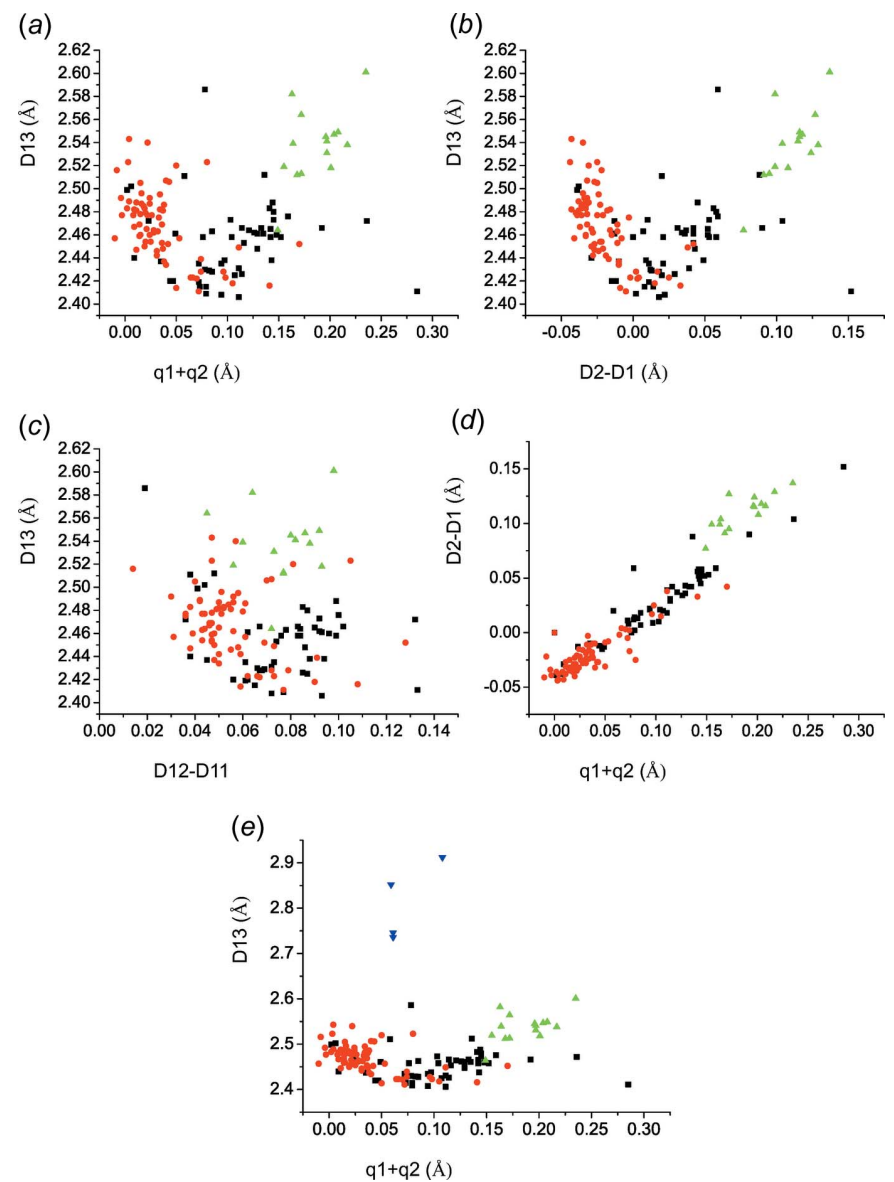

Figure 3

The dependence of distances: (a) $D 13$ on $(q 1+q 2)$; (b) $D 13$ on $D 2-D 1$; (c) $D 13$ on $D 12-D 11 ;(d) D 2-D 1$ on $(q 1+q 2)$; (e) $D 13$ on $(q 1+q 2)$, also for the structures with 3,5-dinitro-2-oxidobenzoate (IV), which are shown as blue triangles. Colour code for symbols: green triangles refer to the structures with 2-hydroxy-3,5-dinitrobenzoic acid (I), black squares are the structures with 2-hydroxy-3,5-dinitrobenzoate (II), and red circles are the structures with 2-carboxy-4,6-dinitrophenolates (III).

bridging hydrogen or the acid hydrogen is believed to affect the positions of the non-hydrogen atoms only minutely, and therefore even the biased structures can be considered further. The parameters $q 1=D 2-D 1$ and $q 2=D 12-D 11$ express the electron delocalization within the fragment $D 1-D 12-D 11-$ $D 2$. The introduction of the parameters $q 1$ and $q 2$ follows an analogous discussion of resonance-assisted hydrogen bonds in the enol forms of $\beta$-diketone fragments (Gilli et al., 1989, 2009). Fig. $3 a$ shows that the distance where the structures with 2-carboxy-4,6-dinitrophenolates (III; red circles) transform into 2-hydroxy-3,5-dinitrobenzoates (II; black squares) corresponds to the shortest distance $D 13_{\min } \simeq 2.41 \AA$, which in turn corresponds to $(q 1+q 2) \simeq 0.08 \AA$. This implies that this is the region where the bridging hydrogen has the greatest tendency to be situated about the centre of the $\mathrm{O} \cdots \mathrm{O}$ intramolecular hydrogen bond or disordered about it. A very similar dependence is shown in Fig. $3 b$, where only distances $D 1$ and $D 3$ are compared. The observed dependence means that the elongation of one $\mathrm{C}-\mathrm{O}$ bond takes place mostly at the cost of the shortening of the neighbouring $\mathrm{C}=\mathrm{O}$ bond; in other words, the distance between these two $\mathrm{O}$ atoms, 
$\mathrm{D} 13 \simeq\left[\left(D 13_{\min }\right)^{2}+(D 2-D 1)^{2}\right]^{1 / 2}$ (Fig. 1). Table 4 lists the structures in which the title molecules are present in different forms. In the recalculated structure of SEDKET (Table 2) and e.g. the reported structures of KEZJIJ (Song et al., 2007) and KEZJIJ01 (Smith et al., 2007) that refer to the structure determination of 2-(pyridin-2-yl)pyridinium 2-carboxy-4,6-dinitrophenolate, the bridging hydrogen is attached to the $\mathrm{O}$ atom having the shorter $\mathrm{C}-\mathrm{O}$ bond distance.

Fig. $3 a$ and $3 b$ also show that the bridging hydrogen cannot be situated near the centre of the intramolecular $\mathrm{O} \cdots \mathrm{O}$ hydrogen bond in structures with 2-hydroxy-3,5-dinitrobenzoic acid (I). Fig. $3 c$ shows a similar dependence of $D 13$ on $(D 12-D 11)$. It can be seen that the adjacent $\mathrm{C}-\mathrm{C}$ conjugated bonds are less, but still sensitive to the bonding of the hydroxy hydrogen atom to one of the neighbouring $\mathrm{C}-\mathrm{O}$ groups. These properties indicate that the $\mathrm{O} \cdots \mathrm{H} \cdots \mathrm{O}$ hydrogen bonding with the pertinent $\mathrm{O} \cdots \mathrm{O}$ distance $\mathrm{D} 13$ belongs to the category of resonance-assisted hydrogen bonds (Gilli et al., 1989, 2009; Sobczyk et al., 2005).

Fig. $3 d$ compares both dependences shown in Figs. $3 a$ and $3 b$. It can be seen that the dependence of $(D 2-D 1)$ on $(q 1+q 2)$ is fairly linear. The dependence seems to show the narrowest spread for the 2-hydroxy-3,5-dinitrobenzoates (II),
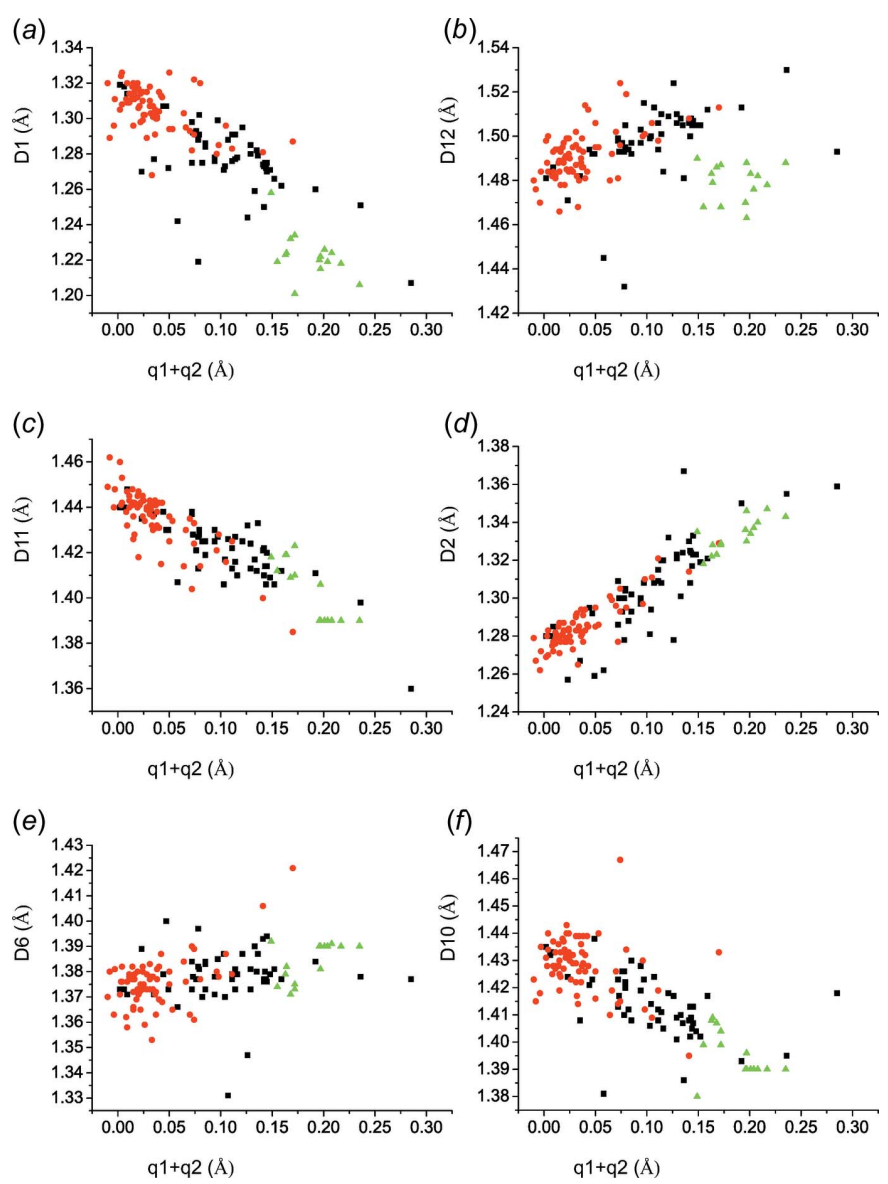

Figure 4

The dependence of bond distances: (a) $D 1$ on $(q 1+q 2)$; (b) $D 12$ on $(q 1+q 2) ;(c) D 11$ on $(q 1+q 2) ;(d) D 2$ on $(q 1+q 2) ;(e) D 6$ on $(q 1+q 2)$; (f) $D 10$ on $(q 1+q 2)$. The colour code for the symbols is the same as in Fig. 3.
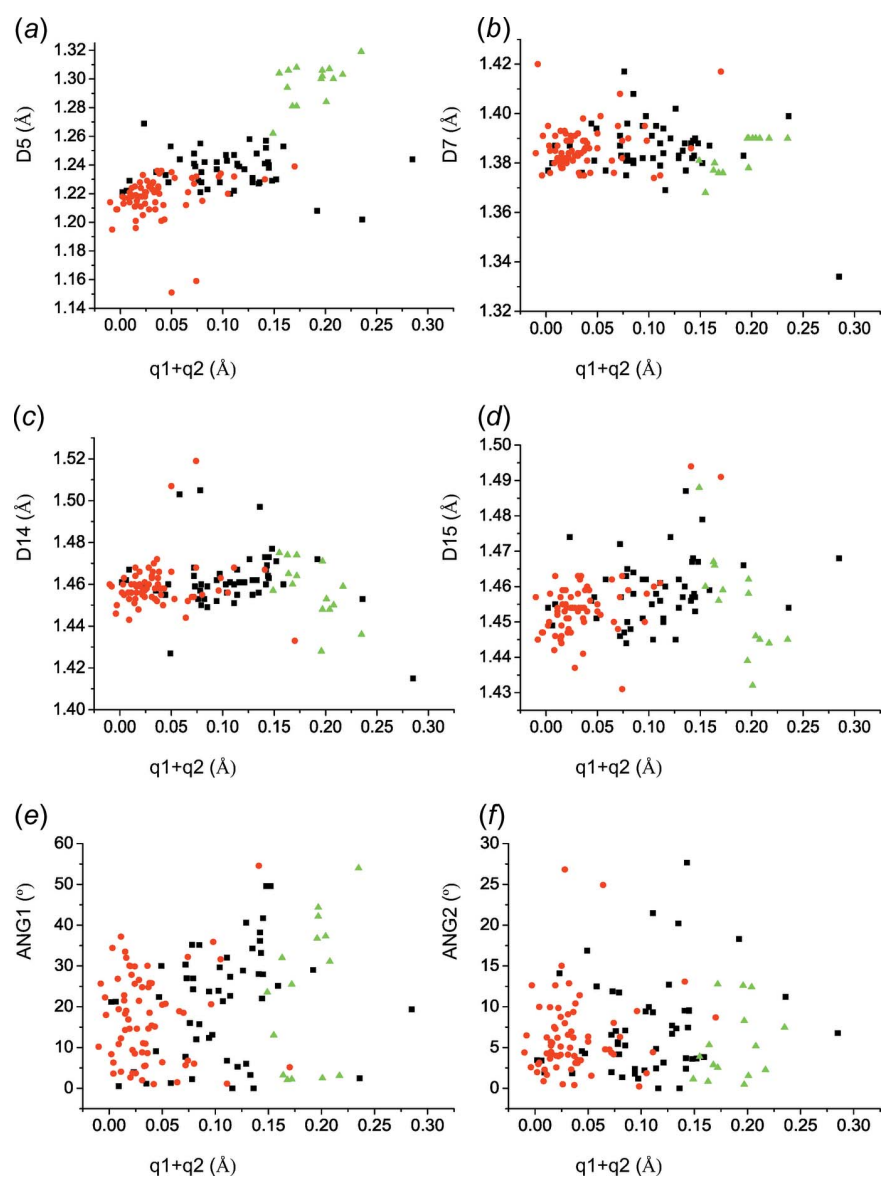

Figure 5

The dependence of bond distances: (a) D5 on $(q 1+q 2)$; $(b) D 7$ on $(q 1+q 2) ;(c) D 14$ on $(q 1+q 2)$. The dependence of dihedral angles: $(e)$ $A N G 1$ on $(q 1+q 2) ;(f) A N G 2$ on $(q 1+q 2)$. [ANG1 and $A N G 2$ are the dihedral angles of the nitro groups involving bonds $D 14$ and $D 15$, respectively, toward the ring plane.] The colour code of the symbols is the same as in Fig. 3.

which are represented by the black squares. Importantly, the line for each class of molecules intercepts the $D 2-D 1$ axis at different values. The structures that contain 2-hydroxy-3,5dinitrobenzoic acid (I) molecules (green triangles) are clearly separated from the rest of the structures although they show a similar trend. Figs. $3 a-3 d$ also show outliers that do not fit the overall trends and which are most probably the structures determined as 2-hydroxy-3,5-dinitrobenzoates (II) instead of 2-carboxy-4,6-dinitrophenolates (III) and vice versa. Fig. $3 e$ shows the same as Fig. $3 a$ except for the addition of a few known structures that contain a 3,5-dinitro-2-oxidobenzoate (IV), which are indicated by blue triangles. Their positions can be explained by the fact that the carboxylate groups are substantially inclined to the benzene ring in such compounds, which causes elongation of the distance between the carboxylate and oxo group, and these molecules will not be considered further.

The alternation of the inclinations (Fig. $4 a-4 d$ ) of the dependences of $D 1, D 12, D 11$, and $D 2$ on $(q 1+q 2)$ are in agreement with the delocalization of the electron density in these bonds. The 2-hydroxy-3,5-dinitrobenzoic acid (I) mol- 
ecules (green triangles) and the 2-hydroxy-3,5-dinitrobenzoates (II; black squares) are situated apart from the 2-carboxy-4,6-dinitrophenolates (III; red circles) in the given figures. The fact that $D 1$ tends to be shortest in 2-hydroxy-3,5dinitrobenzoic acid (I) molecules (Fig. $4 a$ ) can be explained by the elongation of bond $D 5$ in the latter molecules because of the attachment of the hydrogen atom and the concomitant shortening of $D 1$. The bond lengths $D 1$ (Fig. $4 a$ ) are equal to $1.28-1.30 \AA$ at $(q 1+q 2) \simeq 0.08$ where the highest probability for the occurrence of a symmetric intramolecular $\mathrm{O} \cdots \mathrm{H} \cdots \mathrm{O}$ hydrogen bond takes place. The corresponding values of $D 12$, $D 11, D 2, D 6$ and $D 10$ are $1.49 \AA$ (Fig. $4 b$ ), $1.43 \AA$ (Fig. $4 c$ ),

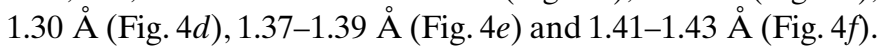

Fig. $5 a$ shows the dependence of D5 on $(q 1+q 2)$. Comparing Fig. $5 a$ to Fig. $4 a$, which shows the dependence of
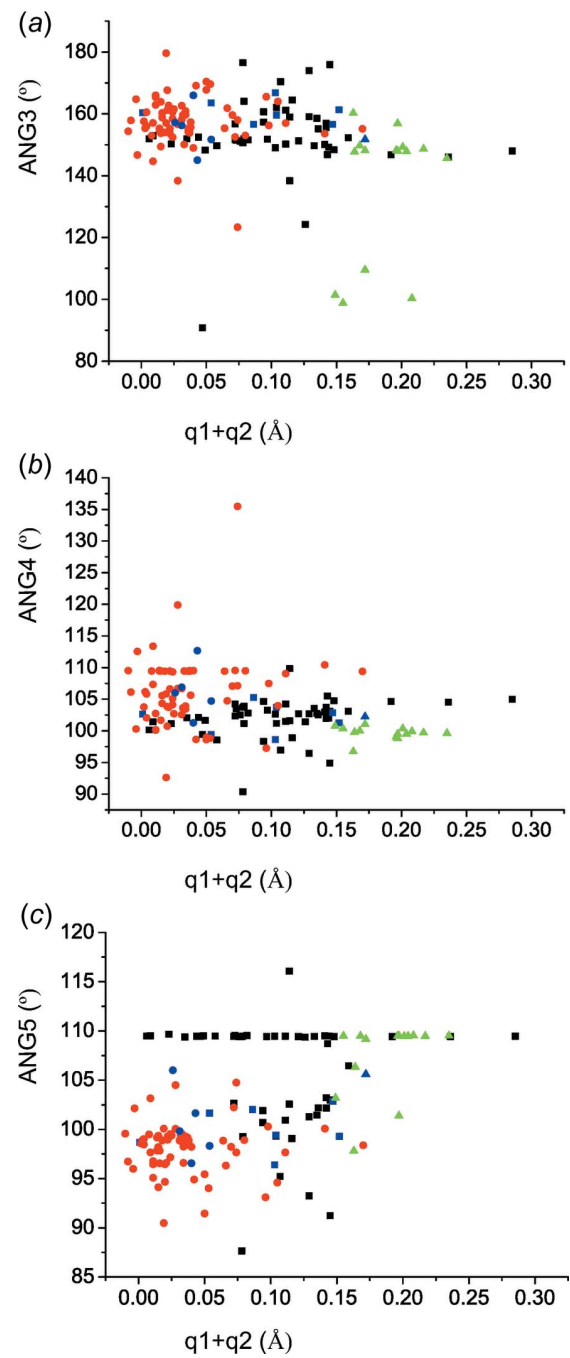

Figure 6

(a) Dependence of the $\mathrm{O} \cdots \mathrm{H} \cdots \mathrm{O}$ angle $A N G 3$ on $(q 1+q 2)$; (b) dependence of $A N G 4$ on $(q 1+q 2)$; (c) dependence of $A N G 5$ on $(q 1+q 2)$. Colour code for symbols: green triangles refer to the structures with 2-hydroxy-3,5-dinitrobenzoic acid (I), black squares are the structures with 2-hydroxy-3,5-dinitrobenzoate (II), and red circles are the structures with 2-carboxy-4,6-dinitrophenolate (III); blue triangles, squares and circles are the recalculated structures with 2-hydroxy-3,5-dinitrobenzoic acid (I), 2-hydroxy-3,5-dinitrobenzoate (II) and 2-carboxy-4,6-dinitrophenolate (III), respectively.
$D 1$ on $(q 1+q 2)$, an indirect proportionality of both dependences can be observed. The bond length $D 5$ is equal to 1.22 $1.24 \AA$ for $(q 1+q 2) \simeq 0.08 \AA$. The dependence of $D 5$ on $(q 1+q 2)$ (Fig. $5 a)$ is similar to that of bond $D 12$ (Fig. $4 b)$ in 2-hydroxy-3,5-dinitrobenzoates (II) and 2-carboxy-4,6-dinitrophenolates (III), but not in molecules of 2-hydroxo-3,5dinitrobenzoic acid (I). It is interesting that 2-hydroxy-3,5dinitrobenzoic acid (I) molecules are in line with other forms of the title molecules for the dependences in Fig. $5 c$ and Fig. $4 d$. Bond $D 7$ is rather distant from the carboxylic group (Fig. 5b) and the delocalization within the pyridine ring is no longer clear. The same holds for bonds $D 14$ and $D 15$ (Figs. $5 c$ and $5 d)$. Figs. $5 e$ and $5 f$ show the inclinations, $A N G 1$ and $A N G 2$, of the nitro groups involving bonds $D 14$ and $D 15$, respectively, toward the ring plane.

Fig. $6 a-6 c$ show dependences in which the localization of the bridging hydrogen takes place. It seems that the most obtuse angles of $\mathrm{O} \cdots \mathrm{H} \cdots \mathrm{O}(A N G 3)$ occur for $(q 1+q 2)$ in the range $<0.06-0.10>\AA$, i.e. for the shortest distances of $D 13$ $(2.41 \AA)$. It is questionable whether the position of a bridging hydrogen in the transition zone between 2-hydroxy-3,5-dinitrobenzoates (II) and 2-carboxy-4,6-dinitrophenolates (III) facilitates its positional disorder, which occurs e.g. in NUQVEB, because of the impossibility of angle $A N G 3$ approaching $180^{\circ}$. The dependence of the angles $A N G 4$ and ANG5 (Fig. 1b) shows once more the effect of incorrectly applied constraints, which are manifested by values close to $109.54^{\circ}$ ( $c f$. Figs. $2 a$ and $2 b$ ).

The previous discussion has shown the correlations of $D 1$ and $D 5$ on $(q 1+q 2)$ (Figs. $4 a$ and $5 a$, respectively), and the indirect dependence of $D 1$ on $D 5$. Therefore, the position of the bridging hydrogen is expected to be related to the environment of the molecules, i.e. to be dependent on $\Delta \mathrm{p} K_{a}=\mathrm{p} K_{a}$ (base) $-\mathrm{p} K_{a}$ (acid). The value of $\Delta \mathrm{p} K_{a}$ is correlated with the occurrence of a structure where the base and the acid components are not ionized, thus forming a cocrystal $(\Delta<0)$, or ionized forming a salt $\left(\Delta \mathrm{p} K_{a}>3\right)$ (Childs $e t$ al., 2007). It is difficult to predict the form in which the acid and the base are present for $0<\Delta \mathrm{p} K_{a}<3$ (Childs et al., 2007).

In Table 4, the structures are ordered according to ascending values of the $\mathrm{p} K_{a}$ values of the bases, i.e. according to increasing basicity. The corresponding values of $\Delta \mathrm{p} K_{a}$ are compared with $(q 1+q 2)$ and $D 13$. The $\mathrm{p} K_{a}$ of 2-hydroxy-3,5dinitrobenzoic acid (I; 3,5-dinitrosalicylic acid) is reported as 2.18 (Smith \& Wermuth, 2014; Hemamalini \& Fun, 2010a), although a value of 1.53 has been reported in the literature (https://www.chemicalbook.com/ProductMSDSDetail CB9172047_EN.htm). The weakest bases given at the top of Table 4 are not able to deprotonate the title molecule, which remains in the form of 2-hydroxy-3,5-dinitrobenzoic acid (I). On the other hand, the bases with the largest values of $\mathrm{p} K_{a}$ (see the bottom of Table 3 ) are able to deprive the title molecule of the hydroxy and acid hydrogen atoms, so in such cases the resulting molecule would be in the form of 3,5-dinitro-2oxidobenzoate (IV). The compounds with moderate basicities are able to deprotonate the acid hydrogen atom but not the bridging hydrogen; hence, the resulting forms are 2-hydroxy- 
Table 4

Overview of selected structures with different forms of the molecules: 2-hydroxy-3,5-dinitrobenzoic acid (I); 2-hydroxy-3,5-dinitrobenzoate (II); 2-carboxy-4,6-dinitrophenolate (III); 3,5-dinitro-2-oxidobenzoate (IV).

The structures are ordered by ascending $\mathrm{p} K_{a}$ value of the base. The corresponding values of $(q 1+q 2), D 13, D 1, D 2$ and $D 5(c f$. Fig. 1$)$ are also given.

\begin{tabular}{|c|c|c|c|c|c|c|c|c|c|c|c|}
\hline & Refcode & $\begin{array}{l}\text { Base and its form } \\
\text { present in the } \\
\text { structure }\end{array}$ & $\mathrm{p} K_{a}$ & $\Delta \mathrm{p} K_{a}$ & Type & $\begin{array}{l}(q 1+q 2) \\
(\AA)\end{array}$ & $\begin{array}{l}D 13 \\
(\AA)\end{array}$ & $\begin{array}{l}D 1 \\
(\AA)\end{array}$ & $\begin{array}{l}D 2 \\
(\AA)\end{array}$ & $\begin{array}{l}D 5 \\
(\AA)\end{array}$ & Remarks \\
\hline 1 & GORXA $M^{a}$ & 1,4-dioxane & -3.9 & -6.08 & $\mathbf{I}$ & $\begin{array}{r}0.204 \\
0.196\end{array}$ & $\begin{array}{l}2.547 \\
2.545\end{array}$ & $\begin{array}{l}1.219 \\
1.220\end{array}$ & $\begin{array}{l}1.337 \\
1.336\end{array}$ & $\begin{array}{l}1.307 \\
1.300\end{array}$ & Two independent molecules \\
\hline 2 & GORXEQ $^{a}$ & 1,4-dioxane & -3.9 & -6.08 & I & 0.235 & 2.601 & 1.206 & 1.343 & 1.319 & \\
\hline 3 & GORXEQ01 $^{a}$ & 1,4-dioxane & -3.9 & -6.08 & I & 0.197 & 2.531 & 1.222 & 1.346 & 1.302 & \\
\hline 4 & $\mathrm{AJEBOG}^{b}$ & $\begin{array}{l}\text { 4-cyano- } \\
\text { pyridinium }\end{array}$ & 1.92 & -0.26 & III & 0.003 & 2.523 & 1.324 & 1.28 & 1.213 & \\
\hline 5 & ABULA $M^{c}$ & $\begin{array}{l}\text { 2-amino- } \\
\text { anilinium }\end{array}$ & $<2$ & $<-0.18$ & III & 0.011 & 2.447 & 1.309 & 1.282 & 1.219 & \\
\hline 6 & PIDCAI $^{c}$ & $\begin{array}{l}\text { 2-amino- } \\
\text { anilinium }\end{array}$ & $<2$ & $<-0.18$ & III & 0.009 & 2.44 & 1.314 & 1.285 & 1.229 & $\begin{array}{l}\text { Wrongly attached hydrogen due to } \mathrm{C}=\mathrm{O} \\
\text { distances. Originally determined as type II but } \\
\text { it should be III. }\end{array}$ \\
\hline 7 & $\operatorname{PERBA} R^{d}$ & $\begin{array}{l}\text { 3-carbamoyl- } \\
\text { pyridinium }\end{array}$ & 3.35 & 1.2 & II & 0.17 & 2.452 & 1.287 & 1.329 & 1.239 & $\begin{array}{l}\text { Wrongly attached hydrogen due to } \mathrm{C}=\mathrm{O} \\
\text { distances. Originally determined as type II but } \\
\text { it is probably III. Disorder present in the } \\
\text { structure. }\end{array}$ \\
\hline 8 & GIFMUE $^{e}$ & $\begin{array}{l}\text { 1-naphthyl- } \\
\text { ammonium }\end{array}$ & 3.92 & 1.74 & III & 0.011 & 2.488 & 1.31 & 1.279 & 1.224 & \\
\hline 9 & MIPROS $^{f}$ & $\begin{array}{l}\text { 8-amino- } \\
\text { quinolinium }\end{array}$ & 3.95 & 1.77 & II & 0.072 & 2.408 & 1.278 & 1.300 & 1.237 & The bridging hydrogen is situated about the centre. \\
\hline 10 & $\mathrm{ABUKUF}^{g}$ & $\begin{array}{l}\text { 4-chloro- } \\
\text { anilinium }\end{array}$ & 3.98 & 1.80 & II & 0.094 & 2.435 & 1.276 & 1.297 & 1.242 & \\
\hline 11 & YIVHIW $^{h}$ & $\begin{array}{l}\text { 4-iodo- } \\
\text { anilinium }\end{array}$ & 4.18 & 1.63 & II & 0.129 & 2.461 & 1.285 & 1.321 & 1.228 & \\
\hline 12 & GIFNUF $^{i}$ & $\begin{array}{l}\text { 1,10-phenan- } \\
\text { throlinium }\end{array}$ & 4.27 & 2.09 & II & 0.096 & 2.428 & 1.280 & 1.297 & 1.232 & $\begin{array}{l}\text { Determined as the type III but it is probably II } \\
\text { (Fig. 1). The chemical name was correct. }\end{array}$ \\
\hline 13 & FOXHAD $^{j}$ & $\begin{array}{l}\text { 2-(pyridin-2-yl)- } \\
\text { pyridinium }\end{array}$ & 4.33 & 2.15 & II & 0.047 & 2.42 & 1.307 & 1.292 & 1.228 & $\begin{array}{l}100 \mathrm{~K} \text {; the reported hydrogen } \mathrm{H} 3 \text { is situated out of } \\
\text { the plane formed by } \mathrm{C} \cdots \mathrm{O} \text { bonds and is } \\
\text { superficial. }\end{array}$ \\
\hline 14 & $\mathrm{KEZJIJ}^{j}$ & $\begin{array}{l}\text { 2-(pyridin-2-yl)- } \\
\text { pyridinium }\end{array}$ & 4.33 & 2.15 & III & 0.07 & 2.422 & 1.293 & 1.296 & 1.231 & $\begin{array}{l}\mathrm{C}=\mathrm{O} \text { distances are about equal. The recalculation } \\
\text { has shown that the bridging hydrogen is about } \\
\text { the centre of the hydrogen bond, slightly closer } \\
\text { to atom } \mathrm{O} 2 \text {, which forms a shorter } \mathrm{C}=\mathrm{O} \text { bond. }\end{array}$ \\
\hline 15 & KEZJIJ01 $1^{j}$ & $\begin{array}{l}\text { 2-(pyridin-2-yl)- } \\
\text { pyridinium }\end{array}$ & 4.33 & 2.15 & III & 0.066 & 2.423 & 1.295 & 1.299 & 1.221 & $\begin{array}{l}\mathrm{C}=\mathrm{O} \text { distances are about equal, the hydrogen is } \\
\text { attached to the } \mathrm{O} \text { atom forming a shorter } \\
\mathrm{C}=\mathrm{O} \text { bond. }\end{array}$ \\
\hline 16 & FICXIZ $^{k}$ & cytosinium & 4.60 & 2.42 & II & 0.098 & 2.423 & 1.285 & 1.310 & 1.234 & $\begin{array}{l}\text { The type according to the } \mathrm{C}=\mathrm{O} \text { distances should } \\
\text { be II; the bridging hydrogen was wrongly } \\
\text { attached. }\end{array}$ \\
\hline 17 & ABUJUE $^{l}$ & anilinium & 4.60 & 2.42 & II & 0.129 & 2.448 & 1.280 & 1.323 & 1.231 & \\
\hline 18 & $\mathrm{ABUKO}^{m}$ & $\begin{array}{l}\text { 4-fluoro- } \\
\text { anilinium }\end{array}$ & 4.65 & 2.47 & II & 0.142 & 2.465 & 1.273 & 1.325 & 1.252 & \\
\hline 19 & GIFMOY $^{n}$ & quinolinium & 4.85 & 2.67 & III & 0.05 & 2.414 & 1.294 & 1.285 & 1.235 & $\begin{array}{l}\text { The title molecule has similarly long } \mathrm{C}=\mathrm{O} \\
\text { distances. }\end{array}$ \\
\hline 20 & ZAJHAT $^{o}$ & $\begin{array}{l}\text { 2-ammino- } \\
\text { benzoic acid }\end{array}$ & 4.96 & 2.78 & II & 0.135 & 2.461 & 1.282 & 1.324 & 1.227 & \\
\hline 21 & AJEBIA $^{p}$ & pyridinium & 5.23 & 3.05 & I and II & $\begin{array}{l}0.142 \\
0.163\end{array}$ & $\begin{array}{l}2.458 \\
2.582\end{array}$ & 1.250 & 1.308 & 1.257 & Two independent molecules \\
\hline 22 & $\mathrm{EGABOF}^{q}$ & $\begin{array}{l}\text { 2-methyl- } \\
\text { quinolinium }\end{array}$ & 5.71 & 3.53 & II & 0.285 & 2.411 & 1.207 & 1.359 & 1.244 & Outlier \\
\hline 23 & AJECEX $01^{r}$ & $\begin{array}{l}\text { 2,6-diamino- } \\
\text { pyridin-1-ium }\end{array}$ & 6.13 & 3.95 & II & $\begin{array}{r}0.072 \\
0.121\end{array}$ & $\begin{array}{l}2.435 \\
2.464\end{array}$ & $\begin{array}{l}1.298 \\
1.295\end{array}$ & $\begin{array}{l}1.309 \\
1.332\end{array}$ & $\begin{array}{l}1.241 \\
1.237\end{array}$ & $\begin{array}{l}\text { One of the title molecules has similarly long } \\
\mathrm{C}=\mathrm{O} \text { distances. }\end{array}$ \\
\hline 24 & $\mathrm{AJECIB}^{s}$ & $\begin{array}{l}\text { 2-amino- } \\
\text { pyrimidinium }\end{array}$ & 6.82 & 4.64 & II & $\begin{array}{l}0.114 \\
0.145\end{array}$ & $\begin{array}{l}2.466 \\
2.473\end{array}$ & $\begin{array}{l}1.277 \\
1.270\end{array}$ & $\begin{array}{l}1.308 \\
1.323\end{array}$ & $\begin{array}{l}1.241 \\
1.238\end{array}$ & \\
\hline 25 & TUMWAB $^{t}$ & $\begin{array}{l}1 H \text {-imidazol- } \\
\text { 3-ium }\end{array}$ & 6.95 & 4.77 & III & -0.01 & 2.457 & 1.320 & 1.279 & 1.214 & \\
\hline 26 & LUMJOU $^{u}$ & hydrazinium & 8.12 & 5.94 & III & 0.014 & 2.459 & 1.318 & 1.275 & 1.211 & \\
\hline 27 & SEDKET $^{v}$ & $\begin{array}{l}\text { 3,5-dimethyl- } \\
\text { pyrazolium }\end{array}$ & 9 & 6.82 & III & 0.037 & 2.481 & 1.300 & 1.282 & 1.224 & \\
\hline 28 & $\begin{array}{l}\text { SEDKET }^{v} \\
\quad(\text { corrected })\end{array}$ & $\begin{array}{l}\text { 3,5-dimethyl- } \\
\text { pyrazolium }\end{array}$ & 9 & 6.82 & II & 0.027 & 2.476 & 1.305 & 1.277 & 1.229 & $\begin{array}{l}\text { The bridging hydrogen after recalculation is closer } \\
\text { to oxygen } \mathrm{O} 1 \text {, which forms the shorter } \mathrm{C}=\mathrm{O} \\
\text { bond }(\mathrm{C} 12-\mathrm{O} 1) \text {. }\end{array}$ \\
\hline 29 & LUDDET $^{w}$ & $\begin{array}{l}\text { benzyl- } \\
\text { ammonium }\end{array}$ & 9.33 & 7.15 & III & 0.002 & 2.483 & 1.305 & 1.269 & 1.218 & \\
\hline 30 & LUDDET01 $^{w}$ & $\begin{array}{l}\text { benzyl- } \\
\text { ammonium }\end{array}$ & 9.33 & 7.15 & III & & & $\begin{array}{l}1.311 \\
1.311\end{array}$ & $\begin{array}{l}1.275 \\
1.279\end{array}$ & $\begin{array}{l}1.217 \\
1.219\end{array}$ & \\
\hline
\end{tabular}


Table 4 (continued)

\begin{tabular}{|c|c|c|c|c|c|c|c|c|c|c|c|}
\hline & Refcode & $\begin{array}{l}\text { Base and its form } \\
\text { present in the } \\
\text { structure }\end{array}$ & $\mathrm{p} K_{a}$ & $\Delta \mathrm{p} K_{a}$ & Type & $\begin{array}{l}(q 1+q 2) \\
(\AA)\end{array}$ & $\begin{array}{l}D 13 \\
(\AA)\end{array}$ & $\begin{array}{l}D 1 \\
(\AA)\end{array}$ & $\begin{array}{l}D 2 \\
(\AA)\end{array}$ & $\begin{array}{l}D 5 \\
(\AA)\end{array}$ & Remarks \\
\hline 31 & INELUI $^{x}$ & $\begin{array}{l}\text { 1-phenylethyl- } \\
\text { ammonium }\end{array}$ & 9.79 & 7.61 & III & $\begin{array}{r}0.009 \\
0.009\end{array}$ & $\begin{array}{l}2.467 \\
2.482\end{array}$ & $\begin{array}{l}1.309 \\
1.320\end{array}$ & $\begin{array}{l}1.272 \\
1.277\end{array}$ & $\begin{array}{l}1.221 \\
1.214\end{array}$ & \\
\hline 32 & MILLOI $^{y}$ & $\begin{array}{l}\text { dicyclohexyl- } \\
\text { ammonium }\end{array}$ & 10.4 & 8.22 & III & 0.028 & 2.464 & 1.289 & 1.273 & 1.225 & $\begin{array}{l}\text { The } \mathrm{C}=\mathrm{O} \text { distances of the title molecule are } \\
\text { similar. }\end{array}$ \\
\hline 33 & ACIFAT $^{z}$ & $\begin{array}{l}\text { 4-sulfamoyl- } \\
\text { anilinium }\end{array}$ & 10.6 & 8.42 & III & 0.028 & 2.462 & 1.315 & 1.287 & 1.209 & \\
\hline 35 & EGUTOP $^{b b}$ & $\begin{array}{l}\text { triethyl- } \\
\text { ammonium }\end{array}$ & 10.78 & 8.6 & II & 0.082 & 2.429 & 1.275 & 1.286 & 1.248 & \\
\hline 36 & EGUTOP01 ${ }^{b b}$ & $\begin{array}{l}\text { triethyl- } \\
\text { ammonium }\end{array}$ & 10.78 & 8.6 & II & 0.072 & 2.419 & 1.275 & 1.288 & 1.242 & \\
\hline 37 & FOGZIL $^{c c}$ & $\begin{array}{l}\text { diethyl- } \\
\text { ammonium }\end{array}$ & 11.09 & 8.91 & III & 0.004 & 2.489 & 1.308 & 1.270 & 1.217 & \\
\hline 38 & XEBFA $M^{d d}$ & $\begin{array}{l}\text { piperidinium } \\
\mathrm{C}_{5} \mathrm{H}_{11} \mathrm{~N}\end{array}$ & 11.28 & 9.1 & II and IV & $\begin{array}{l}0.078 \\
0.061\end{array}$ & $\begin{array}{l}2.586 \\
2.736\end{array}$ & $\begin{array}{l}1.219 \\
1.234\end{array}$ & $\begin{array}{l}1.278 \\
1.253\end{array}$ & $\begin{array}{l}1.255 \\
1.271\end{array}$ & $\begin{array}{l}\text { One molecule of DNSA (I) is fully ionized, } \\
\text { the other is in form II. }\end{array}$ \\
\hline
\end{tabular}

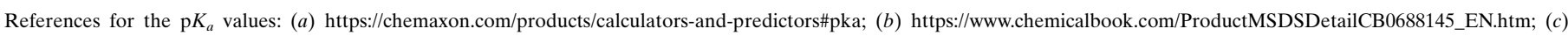

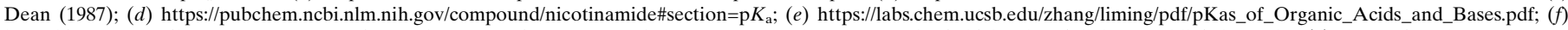

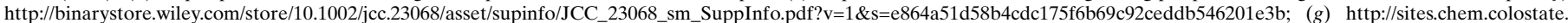

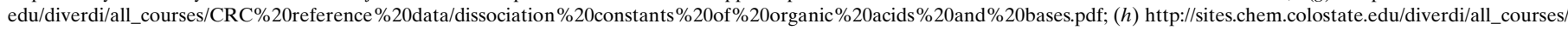

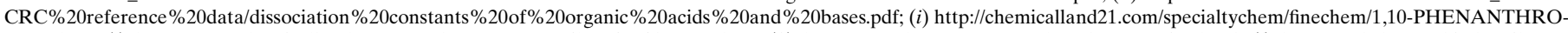

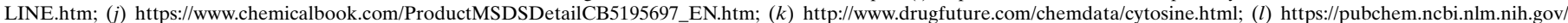

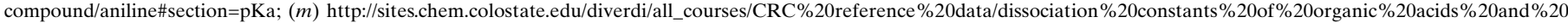

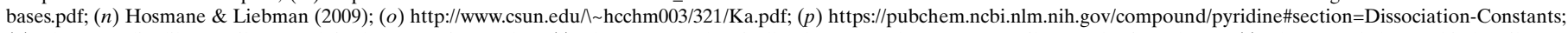

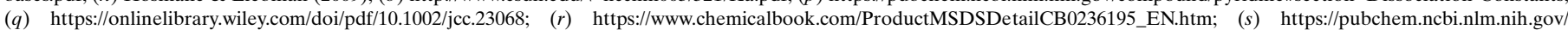

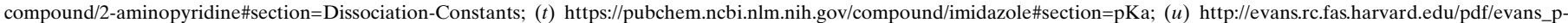

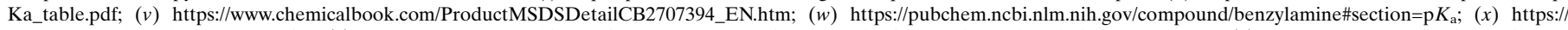

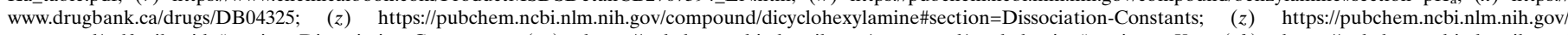

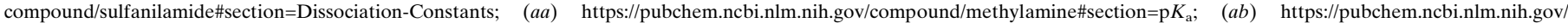

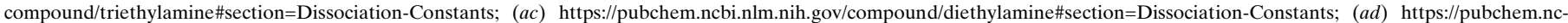

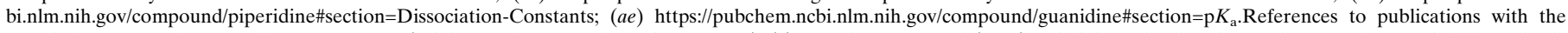

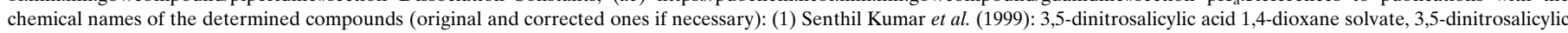

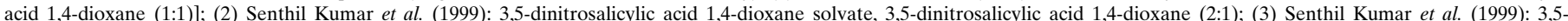

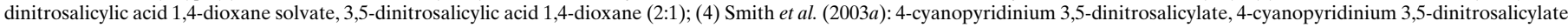

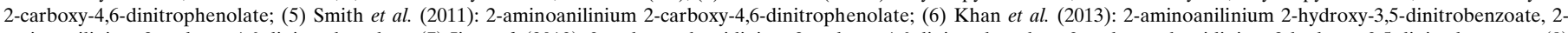

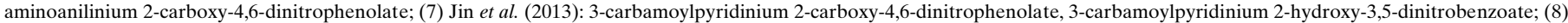

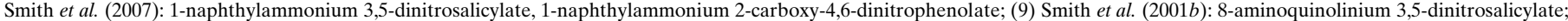

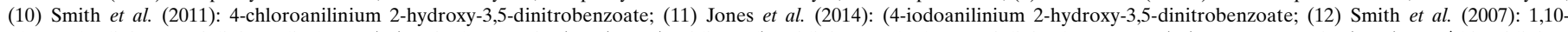

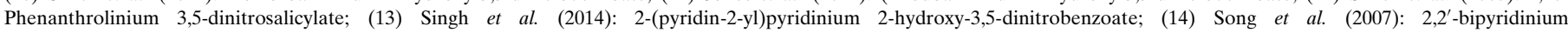

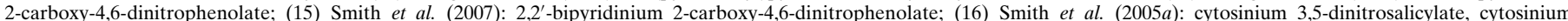

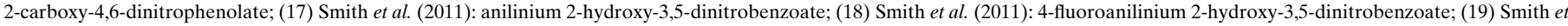

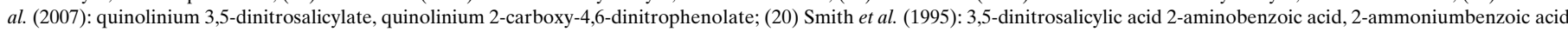

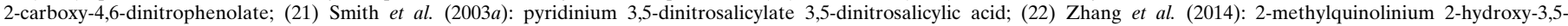

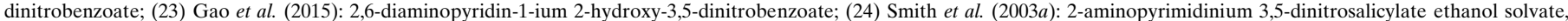

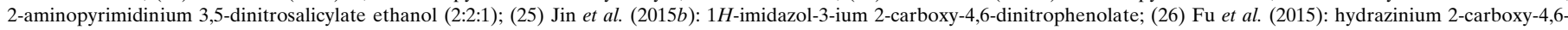

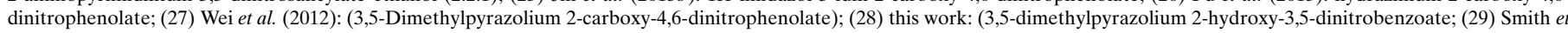

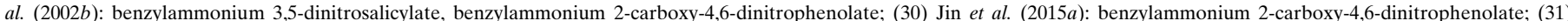

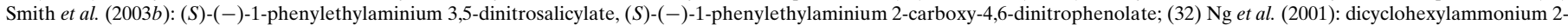

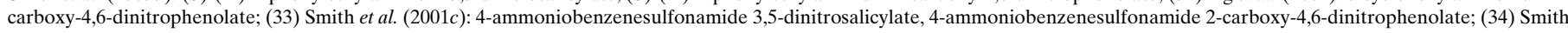

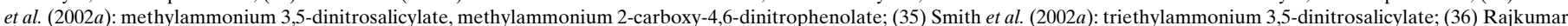

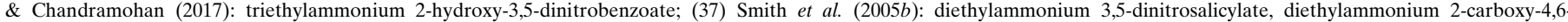

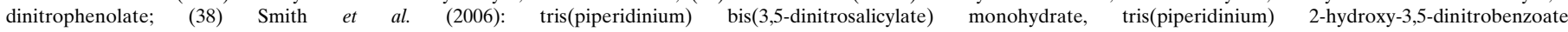
2-olate-3,5-dinitrobenzoate monohydrate; (39) Smith et al. (2001a): guanidinium 3,5-dinitrosalicylate; (40) Fu et al. (2015): guanidinium 3,5-dinitrosalicylate.

3,5-dinitrobenzoate (II) or 2-carboxy-4,6-dinitrophenolate (III). These structures appear in the intermediate region of Table 4. A more radical transfer of the acid hydrogen atom should cause a more significant shortening of bond D5, which should be concomitant with the elongation of bond $D 1$. Such an elongation of bond $D 1$ ( $c f$. Fig. 1a) should support the formation of a 2-carboxy-4,6-dinitrophenolate (III).

\section{Summary}

(1) The bridging hydrogen in the molecules discussed (I-III) is involved in a resonance-assisted hydrogen bond, which is part of a hexagonal $R_{1}^{1}(6)$ ring. The system of conjugated bonds in the title molecules, however, comprises more atoms than the ring in which the bridging hydrogen is involved. In particular, the whole carboxylate/carboxylic group affects the discussed intramolecular $\mathrm{O} \cdots \mathrm{H} \cdots \mathrm{O}$ hydrogen bond.

(2) The transition region between the forms of 2-hydroxy3,5-dinitrobenzoates (II) and 2-carboxy-4,6-dinitrophenolates (III) takes place for $\mathrm{C}-\mathrm{O}(D 1) \simeq 1.28-1.30 \AA, \mathrm{C}-\mathrm{O}(D 2) \simeq$ $1.30 \AA$, $\mathrm{O} \cdots$ O distance $D 13 \simeq 2.41 \AA$ and $(q 1+q 2) \simeq 0.08 \AA$. Simultaneously, the highest probability for the presence of the bridging hydrogen to be in the centre of the hydrogen bond is expected in this transition region. However, the hydrogen 
atom can also be disordered over two positions as occurs in NUQVEB.

(3) The bridging hydrogen in the discussed intramolecular hydrogen bond can be situated at the centre between both oxygen atoms with approximately equal $\mathrm{C}-\mathrm{O}$ bond distances. Therefore, the bridging hydrogen can not be situated at the centre of the intramolecular $\mathrm{O} \cdots \mathrm{H} \cdots \mathrm{O}$ hydrogen bond in compounds containing 2-hydroxy-3,5-dinitrobenzoic acid (I).

(4) In some rare cases (e.g. recalculated SEDKET, KEZJIJ and KEZJIJ01), the bridging hydrogen is bonded to the oxygen atom that forms the shorter $\mathrm{C}-\mathrm{O}$ bond distance (Table 3). It would be of interest to see how the localization of the bridging hydrogen develops with changing temperature in such cases.

(5) Table 4 shows the occurrence of the different forms of the molecules (see scheme) and the dependence on basicity. Alhough it would be expected that the increasing basicity should support the occurrence of 2-carboxy-4,6-dinitrophenolates (III) and, of course, for very strong bases, 3,5-dinitro-2-oxidobenzoates (IV), there are many exceptions to this rule.

(6) The positioning of the hydrogen atoms can be affected by the asphericity of the electron density of the donor and acceptor atoms.

(7) It is essential to calculate difference electron-density maps in order to locate correctly the bridging hydrogen atom, and any other hydrogen atoms involved in hydrogen bonding.

(8) The present overview has shown that the application of constraints and restraints is frequently incorrect.

\section{Funding information}

The author expresses gratitude for the support provided by Project NPU I - LO1603 of the Ministry of Education of the Czech Republic to the Institute of Physics of the Academy of Sciences of the Czech Republic.

\section{References}

Childs, S. L., Stahly, G. P. \& Park, A. (2007). Mol. Pharm. 4, 323338.

Dayananda, A. S., Yathirajan, H. S., Gerber, T., Hosten, E. \& Betz, R. (2012). Acta Cryst. E68, o1165-o1166.

Dean, J. A. (1987). Handbook of Organic Chemistry, pp. 8-46 New York, NY: McGraw-Hill Book Co.

Fu, X., Liu, X., Sun, P., Zhang, S., Yang, Q., Wei, Q., Xie, G., Chen, S. \& Fan, X. (2015). J. Anal. Appl. Pyrolysis, 114, 79-90.

Gao, X., Zhang, H., Wen, X., Liu, B., Jin, S. \& Wang, D. (2015). J. Mol. Struct. 1093, 82-95.

Gilli, G., Bellucci, F., Ferretti, V. \& Bertolasi, V. (1989). J. Am. Chem. Soc. 111, 1023-1028.

Gilli, G. \& Gilli, P. (2009). The Nature of the Hydrogen Bond, pp. 81147. New York: Oxford University Press.

Gilli, P., Pretto, L., Bertolasi, V. \& Gilli, G. (2009). Acc. Chem. Res. 34, 34-44.

Groom, C. R., Bruno, I. J., Lightfoot, M. P. \& Ward, S. C. (2016). Acta Cryst. B72, 171-179.

Hemamalini, M. \& Fun, H.-K. (2010a). Acta Cryst. E66, o1194o1195.

Hemamalini, M. \& Fun, H.-K. (2010b). Acta Cryst. E66, o2950o2951.
Hemamalini, M. \& Fun, H.-K. (2010c). Acta Cryst. E66, o2747.

Hosmane, R. S. \& Liebman, J. F. (2009). Struct. Chem. 20, 693697.

Huang, F., Song, W.-D. \& Li, S.-D. (2007). Acta Cryst. E63, m388m389.

Jeffrey, G. A. (1995). Crystallogr. Rev. 4, 213-254.

Jin, S., Lin, Z., Wang, D., Chen, G., Ji, Z., Huang, T. \& Zhou, Y. (2015a). J. Chem. Crystallogr. 45, 159-168.

Jin, S., Wang, D., Linhe, Q., Fu, M., Wu, S. \& Ren, J. (2013). J. Chem. Crystallogr. 43, 258-265.

Jin, S., Zhang, H., Liu, H., Wen, X., Li, M. \& Wang, D. (2015b). J. Mol. Struct. 1096, 157-170.

Jones, C. L., Wilson, C. C. \& Thomas, L. H. (2014). CrystEngComm, 16, 5849-5858.

Khan, I. M., Ahmad, A. \& Ullah, M. F. (2013). Spectrochim. Acta A, 102, 82-87.

Malathy, S., Nirmalram, J. S. \& Muthiah, P. T. (2015). Acta Cryst. E71, 618-620.

Müller, P. (2009). Crystallogr. Rev. 15, 57-83.

Ng, S. W., Naumov, P., Drew, M. G. B., Wojciechowski, G. \& Brzezinski, B. (2001). J. Mol. Struct. 595, 29-37.

Petříček, V., Dušek, M. \& Palatinus, L. (2014). Z. Kristallogr. 229, 345-352.

Rajkumar, M. \& Chandramohan, A. (2017). J. Mol. Struct. 1134, 762769.

Senthil Kumar, V. S., Kuduva, S. S. \& Desiraju, G. R. (1999). J. Chem. Soc. Perkin Trans. 2, pp. 1069-1074.

Senthil Kumar, V. S., Kuduva, S. S. \& Desiraju, G. R. (2002). Acta Cryst. E58, o865-o866.

Sheldrick, G. M. (2008). Acta Cryst. A64, 112-122.

Sheldrick, G. M. (2015). Acta Cryst. C71, 3-8.

Singh, N., Khan, I. M., Ahmad, A. \& Javed, S. (2014). J. Mol. Liq. 191, $142-150$.

Smith, G., Bott, R. C. \& Wermuth, U. D. (2001a). Acta Cryst. E57, o640-o642.

Smith, G. \& Lynch, D. E. (2016). Acta Cryst. E72, 382-386.

Smith, G., Lynch, D. E., Byriel, K. A. \& Kennard, C. H. L. (1995). Aust. J. Chem. 48, 1133-1149.

Smith, G. \& Wermuth, U. D. (2014). Acta Cryst. E70, 430-434.

Smith, G., Wermuth, U. D., Bott, R. C., Healy, P. C. \& White, J. M. (2002a). Aust. J. Chem. 55, 349-356.

Smith, G., Wermuth, U. D., Bott, R. C., White, J. M. \& Willis, A. C. (2001b). Aust. J. Chem. 54, 165-170.

Smith, G., Wermuth, U. D. \& Healy, P. C. (2002b). Acta Cryst. E58, o845-o847.

Smith, G., Wermuth, U. D. \& Healy, P. C. (2005a). Acta Cryst. E61, o746-o748.

Smith, G., Wermuth, U. D. \& Healy, P. C. (2006). Acta Cryst. E62, o610-0613.

Smith, G., Wermuth, U. D., Healy, P. C. \& White, J. M. (2003a). Aust. J. Chem. 56, 707-713.

Smith, G., Wermuth, U. D., Healy, P. C. \& White, J. M. (2007). Aust. J. Chem. 60, 264-277.

Smith, G., Wermuth, U. D., Healy, P. C. \& White, J. M. (2011). J. Chem. Crystallogr. 41, 1649-1662.

Smith, G., Wermuth, U. D. \& White, J. M. (2001c). Acta Cryst. E57, o1036-o1038.

Smith, G., Wermuth, U. D. \& White, J. M. (2003b). Acta Cryst. E59, o1977-o1979.

Smith, G., Wermuth, U. D. \& White, J. M. (2005b). Acta Cryst. E61, o1836-o1838.

Sobczyk, L., Grabowski, S. J. \& Krygowski, T. M. (2005). Chem. Rev. 105, 3513-3560.

Song, W.-D., Guo, X.-X. \& Yu, L. (2007). Acta Cryst. E63, o1890o1891.

Spek, A. L. (2009). Acta Cryst. D65, 148-155.

Sridhar, B., Nanubolu, J. B. \& Ravikumar, K. (2013). Acta Cryst. C69, 1164-1169. 
Subashini, A., Samuel, E., Muthiah, P. T., Bocelli, G. \& Cantoni, A. (2007). Acta Cryst. E63, o4049.

Wang, S.-T., Che, G.-B., Liu, C.-B., Wang, X. \& Liu, L. (2012). Acta Cryst. E68, m76.

Wei, S., Jin, S., Hu, Z., Zhou, Y. \& Zhou, Y. (2012). Acta Cryst. E68, o3117.
Yamuna, T. S., Kaur, M., Anderson, B. J., Jasinski, J. P. \& Yathirajan, H. S. (2014). Acta Cryst. E70, o318-o319.

Zhang, C.-L. \& Jian, F.-F. (2009). Acta Cryst. E65, m1521.

Zhang, J., Jin, S., Tao, L., Liu, B. \& Wang, D. (2014). J. Mol. Struct. 1072, 208-220. 


\section{supporting information}

Acta Cryst. (2018). E74, 1344-1357 [https://doi.org/10.1107/S2056989018011544]

A resonance-assisted intramolecular hydrogen bond in compounds containing 2-hydroxy-3,5-dinitrobenzoic acid and its various deprotonated forms: redetermination of several related structures

Jan Fábry

Computing details

Data collection: SMART (Bruker, 1997) for DUJZAK; SMART (Bruker, 1999) for JEVNAA; CAD-4 Software (EnrafNonius, 1989) for LUDFUL; APEX2 (Bruker, 2009) for NUQVEB, VABZIJ; SMART (Bruker, 2001) for QIQJAD; SMART (Bruker, 2007) for SAFGUD; SMART (Bruker, 2002) for SEDKET; CrysAlis PRO (Agilent, 2012) for TIYZIM; APEX2 (Bruker, 2004) for TUJPEV; CrysAlis PRO (Agilent, 2014) for WADXOR; APEX2 (Bruker, 2010) for YAXPOE. Cell refinement: SAINT (Bruker, 1997) for DUJZAK; SAINT (Bruker, 1999) for JEVNAA; CAD-4 Software (EnrafNonius, 1989) for LUDFUL; SAINT (Bruker, 2009) for NUQVEB, VABZIJ; SAINT (Bruker, 2001) for QIQJAD; SAINT (Bruker, 2007) for SAFGUD; SAINT (Bruker, 2002) for SEDKET; CrysAlis PRO (Agilent, 2012) for TIYZIM; SAINT (Bruker, 2004) for TUJPEV; CrysAlis PRO (Agilent, 2014) for WADXOR; SAINT (Bruker, 2010) for YAXPOE. Data reduction: SAINT (Bruker, 1997) for DUJZAK; SAINT (Bruker, 1999) for JEVNAA; Xtal3.5 (Hall et al., 1995) for LUDFUL; SAINT (Bruker, 2009) for NUQVEB, VABZIJ; SAINT (Bruker, 2001) for QIQJAD; SAINT (Bruker, 2007) for SAFGUD; SAINT (Bruker, 2002) for SEDKET; CrysAlis RED (Agilent, 2012) for TIYZIM; SAINT (Bruker, 2004) for TUJPEV; CrysAlis PRO (Agilent, 2014) for WADXOR; SAINT (Bruker, 2010) for YAXPOE. Program(s) used to solve structure: SHELXS97 (Sheldrick, 2008) for DUJZAK, JEVNAA, LUDFUL, QIQJAD, SEDKET, TUJPEV, YAXPOE; SHELXTL (Sheldrick, 2008) for NUQVEB, VABZIJ; SHELXS-97 (Sheldrick, 2008) for SAFGUD; SUPERFLIP (Palatinus \& Chapuis, 2007) for TIYZIM; SIR92 (Altomare et al., 1993) for WADXOR. Program(s) used to refine structure: JANA2016 (Petricek et al., 2014) for DUJZAK; JANA2006 (Petricek et al., 2014) for JEVNAA, LUDFUL, NUQVEB, QIQJAD, SAFGUD, TIYZIM, TUJPEV, VABZIJ, YAXPOE; JANA2006 (Petricek, 2014) for SEDKET.

Bis(quinolin-8-ol)silver(I) 2-hydroxy-3,5-dinitrobenzoate (DUJZAK)

Crystal data

$\left[\mathrm{Ag}\left(\mathrm{C}_{9} \mathrm{H}_{7} \mathrm{NO}\right)_{2}\right]\left(\mathrm{C}_{7} \mathrm{H}_{3} \mathrm{~N}_{2} \mathrm{O}_{7}\right)$

$M_{r}=625.30$

Monoclinic, $P 2_{1}$

Hall symbol: $\mathrm{P} 2 \mathrm{yb}$

$a=9.0154(18) \AA$

$b=7.6122(15) \AA$

$c=17.138(3) \AA$

$\beta=104.38(3)^{\circ}$

$V=1139.3(4) \AA^{3}$

$Z=2$
$F(000)=628$

$D_{\mathrm{x}}=1.823 \mathrm{Mg} \mathrm{m}^{-3}$

Mo $K \alpha$ radiation, $\lambda=0.71073 \AA$

Cell parameters from 4356 reflections

$\theta=3.6-27.6^{\circ}$

$\mu=0.95 \mathrm{~mm}^{-1}$

$T=293 \mathrm{~K}$

Block, yellow

$0.20 \times 0.15 \times 0.11 \mathrm{~mm}$ 


\section{Data collection}

Bruker SMART CCD area-detector diffractometer

Radiation source: fine-focus sealed tube Graphite monochromator $\varphi$ and $\omega$ scans

10841 measured reflections

4602 independent reflections

\section{Refinement}

Refinement on $F^{2}$

$R[F>3 \sigma(F)]=0.023$

$w R(F)=0.053$

$S=1.34$

4602 reflections

356 parameters

0 restraints

48 constraints

Primary atom site location: structure-invariant direct methods

Secondary atom site location: difference Fourier map

\section{Special details}

Geometry. All esds (except the esd in the dihedral angle between two 1.s. planes) are estimated using the full covariance matrix. The cell esds are taken into account individually in the estimation of esds in distances, angles and torsion angles; correlations between esds in cell parameters are only used when they are defined by crystal symmetry. An approximate (isotropic) treatment of cell esds is used for estimating esds involving 1.s. planes.

Refinement. Refinement of $F^{2}$ against ALL reflections. The weighted $R$-factor $w R$ and goodness of fit $S$ are based on $F^{2}$, conventional $R$-factors $R$ are based on $F$, with $F$ set to zero for negative $F^{2}$. The threshold expression of $F^{2}>\sigma\left(F^{2}\right)$ is used only for calculating $R$-factors $(\mathrm{gt})$ etc. and is not relevant to the choice of reflections for refinement. $R$-factors based on $F^{2}$ are statistically about twice as large as those based on $F$, and $R$-factors based on ALL data will be even larger.

Number of fixed parameters 9.

Fractional atomic coordinates and isotropic or equivalent isotropic displacement parameters $\left(\AA^{2}\right)$

\begin{tabular}{lllll}
\hline & $x$ & $y$ & $z$ & $U_{\text {iso }} * / U_{\text {eq }}$ \\
\hline Ag1 & $0.062197(18)$ & $0.74282(3)$ & $0.668863(11)$ & $0.01868(5)$ \\
O1 & $-0.0993(2)$ & $0.4630(2)$ & $0.64021(12)$ & $0.0202(6)$ \\
O2 & $0.1741(2)$ & $0.5061(2)$ & $0.77196(12)$ & $0.0214(6)$ \\
N1 & $-0.1154(2)$ & $0.7667(3)$ & $0.55616(13)$ & $0.0177(7)$ \\
N2 & $0.2685(2)$ & $0.8371(3)$ & $0.75427(14)$ & $0.0163(7)$ \\
C1 & $-0.1280(3)$ & $0.9181(4)$ & $0.51463(18)$ & $0.0222(9)$ \\
H1a & -0.061241 & 1.009276 & 0.535386 & $0.0267^{*}$ \\
C2 & $-0.2362(3)$ & $0.9448(4)$ & $0.44205(18)$ & $0.0243(9)$ \\
H2a & -0.240923 & 1.051917 & 0.415439 & $0.0291^{*}$ \\
C3 & $-0.3354(3)$ & $0.8130(4)$ & $0.41016(19)$ & $0.0209(9)$ \\
H3a & -0.407848 & 0.829471 & 0.361612 & $0.0251^{*}$ \\
C4 & $-0.3270(3)$ & $0.6506(4)$ & $0.45167(18)$ & $0.0169(8)$ \\
C5 & $-0.4264(3)$ & $0.5079(4)$ & $0.42259(17)$ & $0.0209(9)$ \\
H5a & -0.500744 & 0.518344 & 0.37429 & $0.0251^{*}$ \\
C6 & $-0.4131(3)$ & $0.3554(4)$ & $0.46539(17)$ & $0.0217(9)$
\end{tabular}

4225 reflections with $I>3 \sigma(I)$

$k=-9 \rightarrow 8$

$l=-22 \rightarrow 22$

Hydrogen site location: difference Fourier map

$\mathrm{H}$ atoms treated by a mixture of independent

and constrained refinement

Weighting scheme based on measured s.u.'s $w=$

$\Delta \rho_{\max }=0.44 \mathrm{e} \AA^{-3}$

$\Delta \rho_{\text {min }}=-0.30$ e $\AA^{-3}$

Absolute structure: 1800 of Friedel pairs used in the refinement

Absolute structure parameter: 0.004 (17)
$1 /\left(\sigma^{2}(I)+0.0004 I^{2}\right)$ 


\begin{tabular}{|c|c|c|c|c|}
\hline H6a & -0.477402 & 0.261662 & 0.445311 & $0.026^{*}$ \\
\hline $\mathrm{C} 7$ & $-0.3036(3)$ & $0.3371(3)$ & $0.53948(17)$ & $0.0184(8)$ \\
\hline $\mathrm{H} 7 \mathrm{a}$ & -0.297965 & 0.232672 & 0.568204 & $0.0221^{*}$ \\
\hline $\mathrm{C} 8$ & $-0.2057(3)$ & $0.4717(3)$ & $0.56954(16)$ & $0.0148(8)$ \\
\hline C9 & $-0.2139(3)$ & $0.6331(3)$ & $0.52566(16)$ & $0.0141(8)$ \\
\hline $\mathrm{C} 10$ & $0.3159(3)$ & $0.9993(4)$ & $0.74633(17)$ & $0.0188(9)$ \\
\hline H10a & 0.256892 & 1.069872 & 0.706108 & $0.0225 *$ \\
\hline $\mathrm{C} 11$ & $0.4512(3)$ & $1.0703(4)$ & $0.79567(18)$ & $0.0220(9)$ \\
\hline H11a & 0.480885 & 1.184529 & 0.787703 & $0.0264 *$ \\
\hline $\mathrm{C} 12$ & $0.5373(3)$ & $0.9693(4)$ & $0.85495(18)$ & $0.0210(9)$ \\
\hline $\mathrm{H} 12 \mathrm{a}$ & 0.627346 & 1.01397 & 0.887796 & $0.0252 *$ \\
\hline $\mathrm{C} 13$ & $0.4907(3)$ & $0.7960(3)$ & $0.86712(16)$ & $0.0171(8)$ \\
\hline $\mathrm{C} 14$ & $0.5743(3)$ & $0.6845(4)$ & $0.92853(17)$ & $0.0200(8)$ \\
\hline $\mathrm{H} 14 \mathrm{a}$ & 0.665083 & 0.723938 & 0.962749 & $0.024 *$ \\
\hline $\mathrm{C} 15$ & $0.5225(3)$ & $0.5197(4)$ & $0.93771(17)$ & $0.0206(8)$ \\
\hline $\mathrm{H} 15 \mathrm{a}$ & 0.577789 & 0.447807 & 0.978539 & $0.0247 *$ \\
\hline $\mathrm{C} 16$ & $0.3863(3)$ & $0.4570(3)$ & $0.88620(16)$ & $0.0173(8)$ \\
\hline H16a & 0.35178 & 0.344669 & 0.8937 & $0.0208^{*}$ \\
\hline $\mathrm{C} 17$ & $0.3043(3)$ & $0.5596(3)$ & $0.82528(16)$ & $0.0142(8)$ \\
\hline $\mathrm{C} 18$ & $0.3541(2)$ & $0.7340(5)$ & $0.81443(13)$ & $0.0140(6)$ \\
\hline $\mathrm{O} 3$ & $0.1402(2)$ & $-0.0134(3)$ & $0.92525(13)$ & $0.0259(7)$ \\
\hline $\mathrm{O} 4$ & $-0.4152(2)$ & $-0.3755(3)$ & $0.69139(13)$ & $0.0284(7)$ \\
\hline O5 & $-0.3271(2)$ & $-0.5961(3)$ & $0.76858(13)$ & $0.0271(7)$ \\
\hline O6 & $0.1238(2)$ & $-0.5247(2)$ & $0.98352(12)$ & $0.0211(6)$ \\
\hline O7 & $0.1679(2)$ & $-0.2659(4)$ & $1.03546(11)$ & $0.0286(6)$ \\
\hline O8 & $-0.0981(2)$ & $0.1498(2)$ & $0.70180(12)$ & $0.0237(7)$ \\
\hline O9 & $0.06657(19)$ & $0.2138(2)$ & $0.81885(11)$ & $0.0196(6)$ \\
\hline N3 & $-0.3217(2)$ & $-0.4436(3)$ & $0.74764(14)$ & $0.0173(7)$ \\
\hline $\mathrm{N} 4$ & $0.1088(2)$ & $-0.3645(3)$ & $0.98085(13)$ & $0.0152(7)$ \\
\hline C19 & $-0.1970(3)$ & $-0.3339(4)$ & 0.79254 (18) & $0.0130(8)$ \\
\hline $\mathrm{C} 20$ & $-0.1027(3)$ & $-0.3992(3)$ & $0.86303(15)$ & $0.0126(7)$ \\
\hline $\mathrm{H} 20 \mathrm{a}$ & -0.114934 & -0.512855 & 0.880295 & $0.0151 *$ \\
\hline $\mathrm{C} 21$ & $0.0095(2)$ & $-0.2908(3)$ & $0.90660(15)$ & $0.0114(8)$ \\
\hline $\mathrm{C} 22$ & $0.0326(3)$ & $-0.1188(3)$ & $0.88194(16)$ & $0.0130(8)$ \\
\hline $\mathrm{C} 23$ & $-0.0605(3)$ & $-0.0603(3)$ & $0.80708(16)$ & $0.0129(7)$ \\
\hline $\mathrm{C} 24$ & $-0.1770(3)$ & $-0.1678(3)$ & $0.76332(17)$ & $0.0132(8)$ \\
\hline $\mathrm{H} 24 \mathrm{a}$ & -0.240539 & -0.128661 & 0.715089 & $0.0159 *$ \\
\hline $\mathrm{C} 25$ & $-0.0307(3)$ & $0.1145(3)$ & $0.77245(16)$ & $0.0148(8)$ \\
\hline H1aa & -0.091407 & 0.377732 & 0.663815 & $0.047(14)^{*}$ \\
\hline H2aa & 0.145536 & 0.395004 & 0.783901 & $0.043(11)^{*}$ \\
\hline $\mathrm{H} 3 \mathrm{~b}$ & 0.135279 & 0.095353 & 0.887993 & $0.17(3)^{*}$ \\
\hline
\end{tabular}

Atomic displacement parameters $\left(\AA^{2}\right)$

\begin{tabular}{lllllll}
\hline & $U^{11}$ & $U^{22}$ & $U^{33}$ & $U^{12}$ & $U^{13}$ & $U^{23}$ \\
\hline $\mathrm{Ag} 1$ & $0.01622(8)$ & $0.01722(9)$ & $0.01945(10)$ & $-0.00339(11)$ & $-0.00154(6)$ & $0.00024(11)$ \\
O1 & $0.0219(9)$ & $0.0127(9)$ & $0.0199(10)$ & $-0.0063(8)$ & $-0.0065(8)$ & $0.0058(8)$ \\
O2 & $0.0185(9)$ & $0.0173(9)$ & $0.0231(11)$ & $-0.0065(8)$ & $-0.0049(8)$ & $0.0030(8)$
\end{tabular}




\begin{tabular}{|c|c|c|c|c|c|c|}
\hline $\mathrm{N} 1$ & $0.0190(9)$ & $0.0152(14)$ & $0.0181(11)$ & $-0.0003(11)$ & $0.0033(8)$ & $0.0008(10)$ \\
\hline N2 & $0.0145(10)$ & $0.0161(11)$ & $0.0187(12)$ & $-0.0018(9)$ & $0.0049(9)$ & $0.0005(9)$ \\
\hline $\mathrm{C} 1$ & $0.0254(14)$ & $0.0145(12)$ & $0.0263(16)$ & $-0.0042(12)$ & $0.0056(12)$ & $0.0044(11)$ \\
\hline $\mathrm{C} 2$ & $0.0325(15)$ & $0.0177(13)$ & $0.0239(16)$ & $0.0069(13)$ & $0.0093(12)$ & $0.0104(11)$ \\
\hline $\mathrm{C} 3$ & $0.0214(13)$ & $0.0247(13)$ & $0.0154(15)$ & $0.0059(12)$ & $0.0021(12)$ & $0.0023(11)$ \\
\hline $\mathrm{C} 4$ & $0.0136(12)$ & $0.0220(14)$ & $0.0150(15)$ & $0.0026(11)$ & $0.0031(10)$ & $0.0005(12)$ \\
\hline $\mathrm{C} 5$ & $0.0160(12)$ & $0.0289(15)$ & $0.0156(14)$ & $0.0005(12)$ & $-0.0002(10)$ & $-0.0035(12)$ \\
\hline C6 & $0.0156(12)$ & $0.0247(14)$ & $0.0217(16)$ & $-0.0092(12)$ & $-0.0009(11)$ & $-0.0062(11)$ \\
\hline $\mathrm{C} 7$ & $0.0179(12)$ & $0.0175(14)$ & $0.0187(14)$ & $-0.0039(11)$ & $0.0026(10)$ & $0.0018(11)$ \\
\hline C8 & $0.0139(12)$ & $0.0146(12)$ & $0.0140(13)$ & $0.0001(11)$ & $0.0000(9)$ & $-0.0001(10)$ \\
\hline C9 & $0.0147(12)$ & $0.0142(12)$ & $0.0132(13)$ & $-0.0002(11)$ & $0.0032(9)$ & $0.0016(10)$ \\
\hline $\mathrm{C} 10$ & $0.0208(13)$ & $0.0176(13)$ & $0.0198(15)$ & $-0.0021(12)$ & $0.0084(11)$ & $0.0021(11)$ \\
\hline $\mathrm{C} 11$ & $0.0259(14)$ & $0.0166(13)$ & $0.0254(16)$ & $-0.0085(12)$ & $0.0099(12)$ & $-0.0039(11)$ \\
\hline $\mathrm{C} 12$ & $0.0180(12)$ & $0.0226(14)$ & $0.0235(15)$ & $-0.0108(12)$ & $0.0074(11)$ & $-0.0101(12)$ \\
\hline $\mathrm{C} 13$ & $0.0138(11)$ & $0.0232(14)$ & $0.0156(14)$ & $-0.0032(10)$ & $0.0064(10)$ & $-0.0059(10)$ \\
\hline $\mathrm{C} 14$ & $0.0118(11)$ & $0.0285(14)$ & $0.0182(15)$ & $-0.0034(11)$ & $0.0010(10)$ & $-0.0071(10)$ \\
\hline C15 & $0.0143(12)$ & $0.0281(15)$ & $0.0175(15)$ & $0.0038(12)$ & $0.0002(10)$ & $0.0017(11)$ \\
\hline $\mathrm{C} 16$ & $0.0160(12)$ & $0.0146(12)$ & $0.0208(15)$ & -0.0018 & $0.0035(10)$ & $-0.0001(10)$ \\
\hline $\mathrm{C} 17$ & $0.0107(11)$ & $0.0149(12)$ & $0.0165(14)$ & $-0.0020(10)$ & $0.0025(9)$ & $-0.0031(10)$ \\
\hline C18 & $0.0115(9)$ & $0.0160(11)$ & $0.0156(11)$ & $0.0014(17)$ & $0.0052(8)$ & $0.0001(15)$ \\
\hline $\mathrm{O} 3$ & $0.0215(10)$ & $0.0236(11)$ & $0.0279(12)$ & $-0.0066(9)$ & $-0.0029(9)$ & $0.0016(9)$ \\
\hline $\mathrm{O} 4$ & $0.0212(10)$ & $0.0283(11)$ & $0.0267(12)$ & $-0.0044(9)$ & $-0.0110(8)$ & $-0.0010(9)$ \\
\hline O5 & $0.0295(10)$ & $0.0195(10)$ & $0.0288(12)$ & $-0.0130(9)$ & $0.0004(9)$ & $0.0016(9)$ \\
\hline O6 & $0.0213(9)$ & $0.0158(9)$ & $0.0236(11)$ & $0.0028(8)$ & $0.0007(8)$ & $0.0059(8)$ \\
\hline $\mathrm{O} 7$ & $0.0340(9)$ & $0.0225(9)$ & $0.0194(9)$ & $0.0057(15)$ & $-0.0121(7)$ & $-0.0015(13)$ \\
\hline O8 & $0.0304(11)$ & $0.0156(10)$ & $0.0204(11)$ & $-0.0061(9)$ & $-0.0028(8)$ & $0.0051(8)$ \\
\hline O9 & $0.0209(8)$ & $0.0129(12)$ & $0.0223(10)$ & $-0.0061(8)$ & $0.0003(7)$ & $0.0009(8)$ \\
\hline N3 & $0.0141(10)$ & $0.0190(11)$ & $0.0169(12)$ & $-0.0064(10)$ & $0.0001(9)$ & $-0.0044(9)$ \\
\hline N4 & $0.0115(10)$ & $0.0169(11)$ & $0.0154(12)$ & $0.0012(9)$ & $-0.0003(8)$ & $0.0025(9)$ \\
\hline C19 & $0.0080(11)$ & $0.0164(12)$ & $0.0135(15)$ & $-0.0043(10)$ & $0.0009(10)$ & $-0.0044(11)$ \\
\hline $\mathrm{C} 20$ & $0.0158(12)$ & $0.0082(11)$ & $0.0137(13)$ & $-0.0009(10)$ & $0.0036(9)$ & $0.0004(9)$ \\
\hline $\mathrm{C} 21$ & $0.0098(9)$ & $0.0122(17)$ & $0.0103(11)$ & $0.0048(10)$ & $-0.0012(8)$ & $0.0021(9)$ \\
\hline $\mathrm{C} 22$ & $0.0086(11)$ & $0.0150(13)$ & $0.0147(14)$ & $-0.0009(10)$ & $0.0015(9)$ & $-0.0033(10)$ \\
\hline $\mathrm{C} 23$ & $0.0133(11)$ & $0.0110(12)$ & $0.0134(13)$ & $-0.0001(10)$ & $0.0015(9)$ & $-0.0007(9)$ \\
\hline $\mathrm{C} 24$ & $0.0120(12)$ & $0.0135(13)$ & $0.0142(15)$ & $0.0018(11)$ & $0.0033(10)$ & $0.0004(11)$ \\
\hline $\mathrm{C} 25$ & $0.0153(12)$ & $0.0109(11)$ & $0.0177(14)$ & $0.0006(10)$ & $0.0029(10)$ & $0.0010(10)$ \\
\hline
\end{tabular}

Geometric parameters $\left(\AA,{ }^{o}\right)$

\begin{tabular}{llll}
\hline $\mathrm{O} 1-\mathrm{C} 8$ & $1.347(3)$ & $\mathrm{C} 13-\mathrm{C} 14$ & $1.415(4)$ \\
$\mathrm{O} 1-\mathrm{H} 1 \mathrm{aa}$ & $0.7585(19)$ & $\mathrm{C} 13-\mathrm{C} 18$ & $1.415(3)$ \\
$\mathrm{O} 2-\mathrm{C} 17$ & $1.359(3)$ & $\mathrm{C} 14-\mathrm{H} 14 \mathrm{a}$ & 0.93 \\
$\mathrm{O} 2-\mathrm{H} 2 \mathrm{aa}$ & $0.922(2)$ & $\mathrm{C} 14-\mathrm{C} 15$ & $1.361(4)$ \\
$\mathrm{N} 1-\mathrm{C} 1$ & $1.344(4)$ & $\mathrm{C} 15-\mathrm{H} 15 \mathrm{a}$ & 0.93 \\
$\mathrm{~N} 1-\mathrm{C} 9$ & $1.365(3)$ & $\mathrm{C} 15-\mathrm{C} 16$ & $1.406(3)$ \\
$\mathrm{N} 2-\mathrm{C} 10$ & $1.325(4)$ & $\mathrm{C} 16-\mathrm{H} 16 \mathrm{a}$ & 0.93 \\
$\mathrm{~N} 2-\mathrm{C} 18$ & $1.371(4)$ & $\mathrm{C} 16-\mathrm{C} 17$ & $1.365(4)$ \\
$\mathrm{C} 1-\mathrm{H} 1 \mathrm{a}$ & 0.93 & $\mathrm{C} 17-\mathrm{C} 18$ & $1.429(5)$
\end{tabular}




\begin{tabular}{|c|c|c|c|}
\hline $\mathrm{C} 1-\mathrm{C} 2$ & $1.392(4)$ & $\mathrm{O} 3-\mathrm{C} 22$ & $1.333(3)$ \\
\hline $\mathrm{C} 2-\mathrm{H} 2 \mathrm{a}$ & 0.93 & $\mathrm{O} 3-\mathrm{H} 3 \mathrm{~b}$ & $1.040(2)$ \\
\hline $\mathrm{C} 2-\mathrm{C} 3$ & $1.364(4)$ & $\mathrm{O} 4-\mathrm{N} 3$ & $1.227(3)$ \\
\hline $\mathrm{C} 3-\mathrm{H} 3 \mathrm{a}$ & 0.93 & $\mathrm{O} 5-\mathrm{N} 3$ & $1.219(3)$ \\
\hline $\mathrm{C} 3-\mathrm{C} 4$ & $1.419(4)$ & $\mathrm{O} 6-\mathrm{N} 4$ & $1.226(3)$ \\
\hline $\mathrm{C} 4-\mathrm{C} 5$ & $1.418(4)$ & $\mathrm{O} 7-\mathrm{N} 4$ & $1.215(3)$ \\
\hline $\mathrm{C} 4-\mathrm{C} 9$ & $1.423(4)$ & $\mathrm{O} 8-\mathrm{C} 25$ & $1.242(3)$ \\
\hline $\mathrm{C} 5-\mathrm{H} 5 \mathrm{a}$ & 0.93 & $\mathrm{O} 9-\mathrm{C} 25$ & $1.275(3)$ \\
\hline $\mathrm{C} 5-\mathrm{C} 6$ & $1.362(4)$ & $\mathrm{O} 9-\mathrm{H} 3 \mathrm{~b}$ & $1.4952(19)$ \\
\hline C6-H6a & 0.93 & N3-C19 & $1.458(3)$ \\
\hline $\mathrm{C} 6-\mathrm{C} 7$ & $1.408(4)$ & $\mathrm{N} 4-\mathrm{C} 21$ & $1.473(3)$ \\
\hline $\mathrm{C} 7-\mathrm{H} 7 \mathrm{a}$ & 0.93 & $\mathrm{C} 19-\mathrm{C} 20$ & $1.385(4)$ \\
\hline $\mathrm{C} 7-\mathrm{C} 8$ & $1.367(4)$ & $\mathrm{C} 19-\mathrm{C} 24$ & $1.388(4)$ \\
\hline $\mathrm{C} 8-\mathrm{C} 9$ & $1.433(4)$ & $\mathrm{C} 20-\mathrm{H} 20 \mathrm{a}$ & 0.93 \\
\hline $\mathrm{C} 10-\mathrm{H} 10 \mathrm{a}$ & 0.93 & $\mathrm{C} 20-\mathrm{C} 21$ & $1.373(3)$ \\
\hline $\mathrm{C} 10-\mathrm{C} 11$ & $1.408(4)$ & $\mathrm{C} 21-\mathrm{C} 22$ & $1.408(4)$ \\
\hline $\mathrm{C} 11-\mathrm{H} 11 \mathrm{a}$ & 0.93 & $\mathrm{C} 22-\mathrm{C} 23$ & $1.419(3)$ \\
\hline $\mathrm{C} 11-\mathrm{C} 12$ & $1.354(4)$ & $\mathrm{C} 23-\mathrm{C} 24$ & $1.394(3)$ \\
\hline $\mathrm{C} 12-\mathrm{H} 12 \mathrm{a}$ & 0.93 & $\mathrm{C} 23-\mathrm{C} 25$ & $1.508(4)$ \\
\hline $\mathrm{C} 12-\mathrm{C} 13$ & $1.416(4)$ & $\mathrm{C} 24-\mathrm{H} 24 \mathrm{a}$ & 0.93 \\
\hline $\mathrm{C} 8-\mathrm{O} 1-\mathrm{H} 1 \mathrm{aa}$ & $118.2(2)$ & $\mathrm{C} 13-\mathrm{C} 14-\mathrm{C} 15$ & $120.2(2)$ \\
\hline $\mathrm{C} 17-\mathrm{O} 2-\mathrm{H} 2 \mathrm{aa}$ & $111.60(19)$ & $\mathrm{H} 14 \mathrm{a}-\mathrm{C} 14-\mathrm{C} 15$ & 119.88 \\
\hline $\mathrm{C} 1-\mathrm{N} 1-\mathrm{C} 9$ & $118.3(2)$ & $\mathrm{C} 14-\mathrm{C} 15-\mathrm{H} 15 \mathrm{a}$ & 119.61 \\
\hline $\mathrm{C} 10-\mathrm{N} 2-\mathrm{C} 18$ & $118.4(2)$ & $\mathrm{C} 14-\mathrm{C} 15-\mathrm{C} 16$ & $120.8(2)$ \\
\hline $\mathrm{N} 1-\mathrm{C} 1-\mathrm{H} 1 \mathrm{a}$ & 118.43 & $\mathrm{H} 15 \mathrm{a}-\mathrm{C} 15-\mathrm{C} 16$ & 119.61 \\
\hline $\mathrm{N} 1-\mathrm{C} 1-\mathrm{C} 2$ & $123.1(2)$ & $\mathrm{C} 15-\mathrm{C} 16-\mathrm{H} 16 \mathrm{a}$ & 119.71 \\
\hline $\mathrm{H} 1 \mathrm{a}-\mathrm{C} 1-\mathrm{C} 2$ & 118.43 & $\mathrm{C} 15-\mathrm{C} 16-\mathrm{C} 17$ & $120.6(2)$ \\
\hline $\mathrm{C} 1-\mathrm{C} 2-\mathrm{H} 2 \mathrm{a}$ & 120.19 & $\mathrm{H} 16 \mathrm{a}-\mathrm{C} 16-\mathrm{C} 17$ & 119.71 \\
\hline $\mathrm{C} 1-\mathrm{C} 2-\mathrm{C} 3$ & $119.6(3)$ & $\mathrm{O} 2-\mathrm{C} 17-\mathrm{C} 16$ & $123.8(2)$ \\
\hline $\mathrm{H} 2 \mathrm{a}-\mathrm{C} 2-\mathrm{C} 3$ & 120.19 & $\mathrm{O} 2-\mathrm{C} 17-\mathrm{C} 18$ & $116.0(2)$ \\
\hline $\mathrm{C} 2-\mathrm{C} 3-\mathrm{H} 3 \mathrm{a}$ & 120.26 & $\mathrm{C} 16-\mathrm{C} 17-\mathrm{C} 18$ & $120.2(2)$ \\
\hline $\mathrm{C} 2-\mathrm{C} 3-\mathrm{C} 4$ & $119.5(2)$ & $\mathrm{N} 2-\mathrm{C} 18-\mathrm{C} 13$ & 121.8 \\
\hline $\mathrm{H} 3 \mathrm{a}-\mathrm{C} 3-\mathrm{C} 4$ & 120.27 & $\mathrm{~N} 2-\mathrm{C} 18-\mathrm{C} 17$ & $119.6(2)$ \\
\hline $\mathrm{C} 3-\mathrm{C} 4-\mathrm{C} 5$ & $122.8(2)$ & $\mathrm{C} 13-\mathrm{C} 18-\mathrm{C} 17$ & $118.6(2)$ \\
\hline $\mathrm{C} 3-\mathrm{C} 4-\mathrm{C} 9$ & $117.8(2)$ & $\mathrm{C} 22-\mathrm{O} 3-\mathrm{H} 3 \mathrm{~b}$ & $102.89(19)$ \\
\hline $\mathrm{C} 5-\mathrm{C} 4-\mathrm{C} 9$ & $119.5(2)$ & $\mathrm{C} 25-\mathrm{O} 9-\mathrm{H} 3 \mathrm{~b}$ & $102.84(17)$ \\
\hline $\mathrm{C} 4-\mathrm{C} 5-\mathrm{H} 5 \mathrm{a}$ & 120 & $\mathrm{O} 4-\mathrm{N} 3-\mathrm{O} 5$ & $124.3(2)$ \\
\hline $\mathrm{C} 4-\mathrm{C} 5-\mathrm{C} 6$ & $120.0(2)$ & $\mathrm{O} 4-\mathrm{N} 3-\mathrm{C} 19$ & $117.5(2)$ \\
\hline $\mathrm{H} 5 \mathrm{a}-\mathrm{C} 5-\mathrm{C} 6$ & 120 & $\mathrm{O} 5-\mathrm{N} 3-\mathrm{C} 19$ & $118.2(2)$ \\
\hline $\mathrm{C} 5-\mathrm{C} 6-\mathrm{H} 6 \mathrm{a}$ & 119.4 & $\mathrm{O} 6-\mathrm{N} 4-\mathrm{O} 7$ & $124.2(2)$ \\
\hline $\mathrm{C} 5-\mathrm{C} 6-\mathrm{C} 7$ & $121.2(2)$ & $\mathrm{O} 6-\mathrm{N} 4-\mathrm{C} 21$ & $116.66(19)$ \\
\hline $\mathrm{H} 6 \mathrm{a}-\mathrm{C} 6-\mathrm{C} 7$ & 119.4 & $\mathrm{O} 7-\mathrm{N} 4-\mathrm{C} 21$ & $119.1(2)$ \\
\hline $\mathrm{C} 6-\mathrm{C} 7-\mathrm{H} 7 \mathrm{a}$ & 119.72 & $\mathrm{~N} 3-\mathrm{C} 19-\mathrm{C} 20$ & 118.7 (2) \\
\hline $\mathrm{C} 6-\mathrm{C} 7-\mathrm{C} 8$ & $120.6(2)$ & $\mathrm{N} 3-\mathrm{C} 19-\mathrm{C} 24$ & $118.9(2)$ \\
\hline $\mathrm{H} 7 \mathrm{a}-\mathrm{C} 7-\mathrm{C} 8$ & 119.72 & $\mathrm{C} 20-\mathrm{C} 19-\mathrm{C} 24$ & $122.3(2)$ \\
\hline $\mathrm{O} 1-\mathrm{C} 8-\mathrm{C} 7$ & $123.5(2)$ & $\mathrm{C} 19-\mathrm{C} 20-\mathrm{H} 20 \mathrm{a}$ & 121.04 \\
\hline $\mathrm{O} 1-\mathrm{C} 8-\mathrm{C} 9$ & $116.5(2)$ & $\mathrm{C} 19-\mathrm{C} 20-\mathrm{C} 21$ & $117.9(2)$ \\
\hline
\end{tabular}




$\mathrm{C} 7-\mathrm{C} 8-\mathrm{C} 9$
N1-C9-C4
N1-C9-C8
C4-C9-C 8
N2-C10-H10a
N2-C10-C11
H10a-C10-C11
C10-C11-H11a
C10-C11-C12
H11a-C11-C12
C11-C12-H12a
C11-C12-C13
H12a-C12-C13
C12-C13-C14
C12-C13-C18
C14-C13-C18
C13-C14-H14a

$120.0(2)$

$121.7(2)$

$119.6(2)$

$118.7(2)$

118.33

$123.3(2)$

118.33

120.58

$118.8(3)$

120.58

119.87

$120.3(2)$

119.87

$123.1(2)$

$117.4(2)$

$119.6(3)$

119.88

$\mathrm{H} 20 \mathrm{a}-\mathrm{C} 20-\mathrm{C} 21$
$\mathrm{~N} 4-\mathrm{C} 21-\mathrm{C} 20$
$\mathrm{~N} 4-\mathrm{C} 21-\mathrm{C} 22$
$\mathrm{C} 20-\mathrm{C} 21-\mathrm{C} 22$
$\mathrm{O} 3-\mathrm{C} 22-\mathrm{C} 21$
$\mathrm{O} 3-\mathrm{C} 22-\mathrm{C} 23$
$\mathrm{C} 21-\mathrm{C} 22-\mathrm{C} 23$
$\mathrm{C} 22-\mathrm{C} 23-\mathrm{C} 24$
$\mathrm{C} 22-\mathrm{C} 23-\mathrm{C} 25$
$\mathrm{C} 24-\mathrm{C} 23-\mathrm{C} 25$
$\mathrm{C} 19-\mathrm{C} 24-\mathrm{C} 23$
$\mathrm{C} 19-\mathrm{C} 24-\mathrm{H} 24 \mathrm{a}$
$\mathrm{C} 23-\mathrm{C} 24-\mathrm{H} 24 \mathrm{a}$
$\mathrm{O} 8-\mathrm{C} 25-\mathrm{O} 9$
$\mathrm{O} 8-\mathrm{C} 25-\mathrm{C} 23$
$\mathrm{O} 9-\mathrm{C} 25-\mathrm{C} 23$
$\mathrm{O} 3-\mathrm{H} 3 \mathrm{~b}-\mathrm{O} 9$

121.04

116.7 (2)

120.67 (19)

$122.7(2)$

$122.3(2)$

$120.0(2)$

$117.7(2)$

$120.0(2)$

$120.6(2)$

$119.4(2)$

$119.2(2)$

120.38

120.38

$125.0(2)$

$118.9(2)$

$116.1(2)$

$155.88(12)$

Hydrogen-bond geometry $\left(\AA,{ }^{\circ}\right)$

\begin{tabular}{lllll}
\hline$D-\mathrm{H} \cdots A$ & $D-\mathrm{H}$ & $\mathrm{H} \cdots A$ & $D \cdots A$ & $D-\mathrm{H} \cdots A$ \\
\hline $\mathrm{C} 11-\mathrm{H} 11 a \cdots \mathrm{O}^{\mathrm{i}}$ & 0.93 & 2.48 & $3.335(4)$ & 152 \\
$\mathrm{O} 1-\mathrm{H} 1 a a \cdots \mathrm{O} 8$ & $0.7585(19)$ & $1.859(2)$ & $2.606(3)$ & $167.96(14)$ \\
$\mathrm{O} 2-\mathrm{H} 2 a a \cdots \mathrm{O} 9$ & $0.922(2)$ & $1.727(2)$ & $2.631(3)$ & $166.48(15)$ \\
$\mathrm{O} 3-\mathrm{H} 3 b \cdots \mathrm{O} 9$ & $1.040(2)$ & $1.4952(19)$ & $2.481(3)$ & $155.88(12)$ \\
\hline
\end{tabular}

Symmetry code: (i) $x+1, y+2, z$.

Tetrakis(1H-imidazole- $\left.\kappa \mathrm{N}^{3}\right)$ zinc(II) bis(2-hydroxy-3,5-dinitrobenzoate) (JEVNAA)

Crystal data

$\left[\mathrm{Zn}\left(\mathrm{C}_{3} \mathrm{H}_{4} \mathrm{~N}_{2}\right)_{4}\right]\left(\mathrm{C}_{7} \mathrm{H}_{3} \mathrm{~N}_{2} \mathrm{O}_{7}\right)_{2}$

$M_{r}=791.93$

Monoclinic, $C 2 / c$

Hall symbol: $-\mathrm{C} 2 \mathrm{yc}$

$a=25.0809(15) \AA$

$b=6.7251(4) \AA$

$c=18.9145(10) \AA$

$\beta=97.658(6)^{\circ}$

$V=3161.9(3) \AA^{3}$

$Z=4$

\section{Data collection}

Bruker APEX-II area-detector diffractometer

Radiation source: fine-focus sealed tube Graphite monochromator $\varphi$ and $\omega$ scans

Absorption correction: multi-scan

(SADABS; Bruker, 1999)

$T_{\min }=0.846, T_{\max }=0.918$
$F(000)=1616$

$D_{\mathrm{x}}=1.664 \mathrm{Mg} \mathrm{m}^{-3}$

Mo $K \alpha$ radiation, $\lambda=0.71073 \AA$

Cell parameters from 3242 reflections

$\theta=2.1-26.9^{\circ}$

$\mu=0.87 \mathrm{~mm}^{-1}$

$T=293 \mathrm{~K}$

Platelet, yellow

$0.20 \times 0.18 \times 0.10 \mathrm{~mm}$

20634 measured reflections

3635 independent reflections

2152 reflections with $I>3 \sigma(I)$

$R_{\text {int }}=0.058$

$\theta_{\text {max }}=27.6^{\circ}, \theta_{\text {min }}=1.6^{\circ}$

$h=-32 \rightarrow 31$

$k=-8 \rightarrow 8$

$l=-24 \rightarrow 24$ 


\section{Refinement}

Refinement on $F^{2}$

Least-squares matrix: full

$R[F>3 \sigma(F)]=0.036$

$w R(F)=0.075$

$S=1.23$

3635 reflections

244 parameters

0 restraints

32 constraints

Primary atom site location: structure-invariant direct methods
Secondary atom site location: difference Fourier map

Hydrogen site location: difference Fourier map

$\mathrm{H}$ atoms treated by a mixture of independent and constrained refinement

Weighting scheme based on measured s.u.'s $w=$ $1 /\left(\sigma^{2}(I)+0.0004 I^{2}\right)$

$(\Delta / \sigma)_{\max }=0.007$

$\Delta \rho_{\max }=0.23 \mathrm{e} \AA^{-3}$

$\Delta \rho_{\min }=-0.23$ e $\AA^{-3}$

Extinction correction: B-C type 1 Lorentzian isotropic (Becker \& Coppens, 1974)

Extinction coefficient: 1400 (500)

\section{Special details}

Geometry. All esds (except the esd in the dihedral angle between two 1.s. planes) are estimated using the full covariance matrix. The cell esds are taken into account individually in the estimation of esds in distances, angles and torsion angles; correlations between esds in cell parameters are only used when they are defined by crystal symmetry. An approximate (isotropic) treatment of cell esds is used for estimating esds involving 1.s. planes.

Refinement. Refinement of $\mathrm{F}^{2}$ against ALL reflections. The weighted R-factor $\mathrm{wR}$ and goodness of fit $\mathrm{S}$ are based on $\mathrm{F}^{2}$, conventional R-factors $R$ are based on $F$, with $F$ set to zero for negative $F^{2}$. The threshold expression of $F^{2}>2 \operatorname{sigma}\left(F^{2}\right)$ is used only for calculating R-factors(gt) etc. and is not relevant to the choice of reflections for refinement. R-factors based on $\mathrm{F}^{2}$ are statistically about twice as large as those based on F, and R- factors based on ALL data will be even larger.

Number of fixed parameters: 9

Fractional atomic coordinates and isotropic or equivalent isotropic displacement parameters $\left(\AA^{2}\right)$

\begin{tabular}{lllll}
\hline & $x$ & $y$ & $z$ & $U_{\text {iso }} * U_{\text {eq }}$ \\
\hline C1 & $0.47960(10)$ & $0.4200(3)$ & $0.62639(13)$ & $0.0460(10)$ \\
H1 & 0.444004 & 0.398202 & 0.633096 & $0.0552^{*}$ \\
C2 & $0.50386(10)$ & $0.3369(4)$ & $0.57533(13)$ & $0.0484(10)$ \\
H2 & 0.488745 & 0.248295 & 0.540565 & $0.0581^{*}$ \\
C3 & $0.56018(9)$ & $0.5299(3)$ & $0.63970(12)$ & $0.0401(9)$ \\
H3 & 0.591591 & 0.597819 & 0.656666 & $0.0481^{*}$ \\
C4 & $0.59325(9)$ & $0.9065(4)$ & $0.83991(12)$ & $0.0404(9)$ \\
H4 & 0.591722 & 0.823229 & 0.87885 & $0.0485^{*}$ \\
N4 & $0.62879(7)$ & $1.0508(3)$ & $0.83789(11)$ & $0.0471(8)$ \\
C6 & $0.57665(9)$ & $1.0451(4)$ & $0.73735(13)$ & $0.0444(9)$ \\
H6 & 0.560952 & 1.074705 & 0.691199 & $0.0533^{*}$ \\
C7 & $0.73999(9)$ & $0.4553(3)$ & $0.34225(11)$ & $0.0329(8)$ \\
C8 & $0.79245(9)$ & $0.5059(3)$ & $0.36676(12)$ & $0.0342(8)$ \\
H8 & 0.817639 & 0.51762 & 0.335092 & $0.0411^{*}$ \\
C9 & $0.80717(8)$ & $0.5388(3)$ & $0.43803(12)$ & $0.0311(8)$ \\
C10 & $0.77048(8)$ & $0.5210(3)$ & $0.48978(12)$ & $0.0302(8)$ \\
C11 & $0.71702(8)$ & $0.4593(3)$ & $0.46099(11)$ & $0.0282(7)$ \\
C12 & $0.70286(9)$ & $0.4300(3)$ & $0.38898(12)$ & $0.0323(8)$ \\
H12 & 0.667845 & 0.392676 & 0.371656 & $0.0388^{*}$ \\
C13 & $0.67545(9)$ & $0.4249(3)$ & $0.50834(12)$ & $0.0333(8)$ \\
N1 & $0.51487(7)$ & $0.5429(3)$ & $0.66784(9)$ & $0.0373(7)$ \\
N2 & $0.55523(8)$ & $0.4075(3)$ & $0.58408(10)$ & $0.0438(8)$ \\
& & & &
\end{tabular}




$\begin{array}{lllll}\text { N3 } & 0.56024(7) & 0.8957(3) & 0.77961(9) & 0.0354(7) \\ \text { C5 } & 0.61854(9) & 1.1406(4) & 0.77303(14) & 0.0479(10) \\ \text { H5 } & 0.637064 & 1.247402 & 0.75675 & 0.0575^{*} \\ \text { N5 } & 0.72386(8) & 0.4225(3) & 0.26644(10) & 0.0427(8) \\ \text { N6 } & 0.86351(7) & 0.5897(3) & 0.46068(11) & 0.0403(8) \\ \text { O1 } & 0.78238(6) & 0.5518(2) & 0.55681(8) & 0.0378(6) \\ \text { O2 } & 0.68832(6) & 0.4695(2) & 0.57640(8) & 0.0420(6) \\ \text { O3 } & 0.63104(6) & 0.3579(2) & 0.48669(8) & 0.0426(6) \\ \text { O4 } & 0.67630(7) & 0.3835(3) & 0.24643(8) & 0.0575(7) \\ \text { O5 } & 0.75772(7) & 0.4340(3) & 0.22570(9) & 0.0662(8) \\ \text { O6 } & 0.89634(7) & 0.5475(3) & 0.42013(9) & 0.0658(8) \\ \text { O7 } & 0.87622(6) & 0.6730(3) & 0.51757(9) & 0.0568(7) \\ \text { Zn1 } & 0.5 & 0.70908(6) & 0.75 & 0.03866(15) \\ \text { H1a } & 0.728641 & 0.508268 & 0.579335 & 0.108(11)^{*} \\ \text { H2a } & 0.580668 & 0.367714 & 0.552562 & 0.088(9)^{*} \\ \text { H4a } & 0.657593 & 1.098579 & 0.870594 & 0.106(11)^{*}\end{array}$

Atomic displacement parameters $\left(\AA^{2}\right)$

\begin{tabular}{lllllll}
\hline & $U^{11}$ & $U^{22}$ & $U^{33}$ & $U^{12}$ & $U^{13}$ & $U^{23}$ \\
\hline C1 & $0.0323(14)$ & $0.0558(17)$ & $0.0507(17)$ & $-0.0069(12)$ & $0.0086(12)$ & $-0.0071(13)$ \\
C2 & $0.0460(16)$ & $0.0536(18)$ & $0.0448(17)$ & $-0.0064(13)$ & $0.0033(12)$ & $-0.0116(13)$ \\
C3 & $0.0327(13)$ & $0.0485(16)$ & $0.0396(16)$ & $-0.0053(11)$ & $0.0071(11)$ & $0.0037(12)$ \\
C4 & $0.0366(14)$ & $0.0491(16)$ & $0.0343(15)$ & $0.0066(12)$ & $0.0004(11)$ & $0.0062(12)$ \\
N4 & $0.0332(12)$ & $0.0568(15)$ & $0.0484(15)$ & $-0.0016(10)$ & $-0.0048(10)$ & $-0.0044(11)$ \\
C6 & $0.0416(15)$ & $0.0568(17)$ & $0.0336(15)$ & $-0.0015(12)$ & $0.0004(12)$ & $0.0077(13)$ \\
C7 & $0.0344(13)$ & $0.0369(14)$ & $0.0264(14)$ & $0.0024(10)$ & $0.0002(10)$ & $-0.0008(10)$ \\
C8 & $0.0332(13)$ & $0.0366(14)$ & $0.0337(15)$ & $0.0009(10)$ & $0.0073(10)$ & $0.0030(10)$ \\
C9 & $0.0254(12)$ & $0.0287(13)$ & $0.0382(15)$ & $-0.0031(9)$ & $0.0003(10)$ & $-0.0001(10)$ \\
C10 & $0.0338(13)$ & $0.0219(12)$ & $0.0342(14)$ & $0.0035(9)$ & $0.0013(10)$ & $0.0006(10)$ \\
C11 & $0.0288(12)$ & $0.0245(12)$ & $0.0310(14)$ & $0.0011(9)$ & $0.0030(10)$ & $-0.0013(10)$ \\
C12 & $0.0281(12)$ & $0.0306(14)$ & $0.0370(15)$ & $0.0009(9)$ & $-0.0002(10)$ & $0.0004(10)$ \\
C13 & $0.0338(13)$ & $0.0312(14)$ & $0.0356(15)$ & $0.0037(10)$ & $0.0067(11)$ & $-0.0026(10)$ \\
N1 & $0.0341(11)$ & $0.0433(13)$ & $0.0357(12)$ & $-0.0029(9)$ & $0.0086(9)$ & $-0.0001(9)$ \\
N2 & $0.0437(12)$ & $0.0526(14)$ & $0.0371(13)$ & $0.0034(10)$ & $0.0126(10)$ & $-0.0035(10)$ \\
N3 & $0.0321(10)$ & $0.0442(12)$ & $0.0288(11)$ & $0.0016(9)$ & $0.0003(9)$ & $0.0042(9)$ \\
C5 & $0.0393(15)$ & $0.0540(18)$ & $0.0499(18)$ & $-0.0090(12)$ & $0.0040(12)$ & $0.0069(14)$ \\
N5 & $0.0414(13)$ & $0.0517(14)$ & $0.0338(13)$ & $0.0032(10)$ & $0.0004(10)$ & $-0.0005(10)$ \\
N6 & $0.0327(11)$ & $0.0435(13)$ & $0.0438(14)$ & $-0.0038(9)$ & $0.0023(10)$ & $0.0059(10)$ \\
O1 & $0.0362(9)$ & $0.0445(10)$ & $0.0308(10)$ & $-0.0009(7)$ & $-0.0026(7)$ & $-0.0054(7)$ \\
O2 & $0.0399(10)$ & $0.0552(11)$ & $0.0313(10)$ & $-0.0055(8)$ & $0.0056(7)$ & $-0.0066(8)$ \\
O3 & $0.0325(9)$ & $0.0561(11)$ & $0.0400(10)$ & $-0.0091(8)$ & $0.0079(7)$ & $-0.0084(8)$ \\
O4 & $0.0395(10)$ & $0.0890(14)$ & $0.0408(11)$ & $-0.0047(9)$ & $-0.0063(8)$ & $-0.0048(9)$ \\
O5 & $0.0499(11)$ & $0.1146(17)$ & $0.0361(11)$ & $-0.0057(10)$ & $0.0133(9)$ & $-0.0036(10)$ \\
O6 & $0.0351(10)$ & $0.1012(16)$ & $0.0635(13)$ & $-0.0075(10)$ & $0.0156(9)$ & $-0.0121(11)$ \\
O7 & $0.0430(10)$ & $0.0799(14)$ & $0.0453(11)$ & $-0.0182(9)$ & $-0.0029(8)$ & $-0.0108(10)$ \\
Zn1 & $0.0355(2)$ & $0.0455(3)$ & $0.0352(3)$ & 0 & $0.00566(17)$ & 0
\end{tabular}


Geometric parameters $\left(\AA,{ }^{\circ}\right)$

\begin{tabular}{|c|c|c|c|}
\hline $\mathrm{C} 1-\mathrm{H} 1$ & 0.93 & $\mathrm{C} 8-\mathrm{H} 8$ & 0.93 \\
\hline $\mathrm{C} 1-\mathrm{C} 2$ & $1.331(4)$ & $\mathrm{C} 8-\mathrm{C} 9$ & $1.367(3)$ \\
\hline $\mathrm{C} 1-\mathrm{N} 1$ & $1.377(3)$ & $\mathrm{C} 9-\mathrm{C} 10$ & $1.435(3)$ \\
\hline $\mathrm{C} 2-\mathrm{H} 2$ & 0.93 & $\mathrm{C} 9-\mathrm{N} 6$ & $1.462(3)$ \\
\hline $\mathrm{C} 2-\mathrm{N} 2$ & $1.362(3)$ & $\mathrm{C} 10-\mathrm{C} 11$ & $1.440(3)$ \\
\hline $\mathrm{C} 3-\mathrm{H} 3$ & 0.93 & $\mathrm{C} 10-\mathrm{O} 1$ & $1.280(3)$ \\
\hline $\mathrm{C} 3-\mathrm{N} 1$ & $1.320(3)$ & $\mathrm{C} 11-\mathrm{C} 12$ & $1.375(3)$ \\
\hline $\mathrm{C} 3-\mathrm{N} 2$ & $1.328(3)$ & $\mathrm{C} 11-\mathrm{C} 13$ & $1.480(3)$ \\
\hline $\mathrm{C} 4-\mathrm{H} 4$ & 0.93 & $\mathrm{C} 12-\mathrm{H} 12$ & 0.93 \\
\hline $\mathrm{C} 4-\mathrm{N} 4$ & $1.322(3)$ & $\mathrm{C} 13-\mathrm{O} 2$ & $1.319(3)$ \\
\hline $\mathrm{C} 4-\mathrm{N} 3$ & $1.319(3)$ & $\mathrm{C} 13-\mathrm{O} 3$ & $1.220(3)$ \\
\hline $\mathrm{N} 4-\mathrm{C} 5$ & $1.361(3)$ & $\mathrm{N} 2-\mathrm{H} 2 \mathrm{a}$ & $0.967(2)$ \\
\hline $\mathrm{N} 4-\mathrm{H} 4 \mathrm{a}$ & $0.9427(18)$ & $\mathrm{C} 5-\mathrm{H} 5$ & 0.93 \\
\hline $\mathrm{C} 6-\mathrm{H} 6$ & 0.93 & $\mathrm{~N} 5-\mathrm{O} 4$ & $1.231(3)$ \\
\hline $\mathrm{C} 6-\mathrm{N} 3$ & $1.381(3)$ & $\mathrm{N} 5-\mathrm{O} 5$ & $1.223(3)$ \\
\hline $\mathrm{C} 6-\mathrm{C} 5$ & $1.335(3)$ & N6-O6 & $1.231(3)$ \\
\hline $\mathrm{C} 7-\mathrm{C} 8$ & $1.378(3)$ & $\mathrm{N} 6-\mathrm{O} 7$ & $1.217(3)$ \\
\hline $\mathrm{C} 7-\mathrm{C} 12$ & $1.377(3)$ & $\mathrm{O} 1-\mathrm{H} 1 \mathrm{a}$ & $1.4955(15)$ \\
\hline $\mathrm{C} 7-\mathrm{N} 5$ & $1.454(3)$ & $\mathrm{O} 2-\mathrm{H} 1 \mathrm{a}$ & $1.0386(15)$ \\
\hline $\mathrm{H} 1-\mathrm{C} 1-\mathrm{C} 2$ & 124.94 & $\mathrm{C} 9-\mathrm{C} 10-\mathrm{O} 1$ & $125.18(18)$ \\
\hline $\mathrm{H} 1-\mathrm{C} 1-\mathrm{N} 1$ & 124.94 & $\mathrm{C} 11-\mathrm{C} 10-\mathrm{O} 1$ & $120.2(2)$ \\
\hline $\mathrm{C} 2-\mathrm{C} 1-\mathrm{N} 1$ & $110.1(2)$ & $\mathrm{C} 10-\mathrm{C} 11-\mathrm{C} 12$ & $121.4(2)$ \\
\hline $\mathrm{C} 1-\mathrm{C} 2-\mathrm{H} 2$ & 126.81 & $\mathrm{C} 10-\mathrm{C} 11-\mathrm{C} 13$ & $120.78(19)$ \\
\hline $\mathrm{C} 1-\mathrm{C} 2-\mathrm{N} 2$ & $106.4(2)$ & $\mathrm{C} 12-\mathrm{C} 11-\mathrm{C} 13$ & $117.85(18)$ \\
\hline $\mathrm{H} 2-\mathrm{C} 2-\mathrm{N} 2$ & 126.81 & $\mathrm{C} 7-\mathrm{C} 12-\mathrm{C} 11$ & 120.69 (19) \\
\hline $\mathrm{H} 3-\mathrm{C} 3-\mathrm{N} 1$ & 124.28 & $\mathrm{C} 7-\mathrm{C} 12-\mathrm{H} 12$ & 119.65 \\
\hline $\mathrm{H} 3-\mathrm{C} 3-\mathrm{N} 2$ & 124.28 & $\mathrm{C} 11-\mathrm{C} 12-\mathrm{H} 12$ & 119.66 \\
\hline $\mathrm{N} 1-\mathrm{C} 3-\mathrm{N} 2$ & 111.44 (19) & $\mathrm{C} 11-\mathrm{C} 13-\mathrm{O} 2$ & $117.03(18)$ \\
\hline $\mathrm{H} 4-\mathrm{C} 4-\mathrm{N} 4$ & 124.35 & $\mathrm{C} 11-\mathrm{C} 13-\mathrm{O} 3$ & $122.6(2)$ \\
\hline $\mathrm{H} 4-\mathrm{C} 4-\mathrm{N} 3$ & 124.35 & $\mathrm{O} 2-\mathrm{C} 13-\mathrm{O} 3$ & $120.3(2)$ \\
\hline $\mathrm{N} 4-\mathrm{C} 4-\mathrm{N} 3$ & $111.3(2)$ & $\mathrm{C} 1-\mathrm{N} 1-\mathrm{C} 3$ & $104.66(19)$ \\
\hline $\mathrm{C} 4-\mathrm{N} 4-\mathrm{C} 5$ & $107.74(19)$ & $\mathrm{C} 2-\mathrm{N} 2-\mathrm{C} 3$ & $107.4(2)$ \\
\hline $\mathrm{C} 4-\mathrm{N} 4-\mathrm{H} 4 \mathrm{a}$ & $133.8(2)$ & $\mathrm{C} 2-\mathrm{N} 2-\mathrm{H} 2 \mathrm{a}$ & $121.3(2)$ \\
\hline $\mathrm{C} 5-\mathrm{N} 4-\mathrm{H} 4 \mathrm{a}$ & $118.5(2)$ & $\mathrm{C} 3-\mathrm{N} 2-\mathrm{H} 2 \mathrm{a}$ & $131.3(2)$ \\
\hline $\mathrm{H} 6-\mathrm{C} 6-\mathrm{N} 3$ & 125.29 & $\mathrm{C} 4-\mathrm{N} 3-\mathrm{C} 6$ & $105.04(18)$ \\
\hline $\mathrm{H} 6-\mathrm{C} 6-\mathrm{C} 5$ & 125.29 & $\mathrm{~N} 4-\mathrm{C} 5-\mathrm{C} 6$ & 106.5 \\
\hline $\mathrm{N} 3-\mathrm{C} 6-\mathrm{C} 5$ & $109.4(2)$ & $\mathrm{N} 4-\mathrm{C} 5-\mathrm{H} 5$ & 126.75 \\
\hline $\mathrm{C} 8-\mathrm{C} 7-\mathrm{C} 12$ & $120.8(2)$ & $\mathrm{C} 6-\mathrm{C} 5-\mathrm{H} 5$ & 126.75 \\
\hline $\mathrm{C} 8-\mathrm{C} 7-\mathrm{N} 5$ & $119.8(2)$ & $\mathrm{C} 7-\mathrm{N} 5-\mathrm{O} 4$ & $117.82(19)$ \\
\hline $\mathrm{C} 12-\mathrm{C} 7-\mathrm{N} 5$ & $119.40(19)$ & $\mathrm{C} 7-\mathrm{N} 5-\mathrm{O} 5$ & $119.10(18)$ \\
\hline $\mathrm{C} 7-\mathrm{C} 8-\mathrm{H} 8$ & 120.27 & $\mathrm{O} 4-\mathrm{N} 5-\mathrm{O} 5$ & $123.08(19)$ \\
\hline $\mathrm{C} 7-\mathrm{C} 8-\mathrm{C} 9$ & $119.5(2)$ & $\mathrm{C} 9-\mathrm{N} 6-\mathrm{O} 6$ & $117.66(19)$ \\
\hline $\mathrm{H} 8-\mathrm{C} 8-\mathrm{C} 9$ & 120.27 & $\mathrm{C} 9-\mathrm{N} 6-\mathrm{O} 7$ & $119.73(19)$ \\
\hline $\mathrm{C} 8-\mathrm{C} 9-\mathrm{C} 10$ & $123.06(18)$ & $\mathrm{O} 6-\mathrm{N} 6-\mathrm{O} 7$ & $122.61(18)$ \\
\hline $\mathrm{C} 8-\mathrm{C} 9-\mathrm{N} 6$ & $116.8(2)$ & $\mathrm{C} 10-\mathrm{O} 1-\mathrm{H} 1 \mathrm{a}$ & 98.67 (13) \\
\hline
\end{tabular}



$\mathrm{C} 10-\mathrm{C} 9-\mathrm{N} 6$
$120.16(19)$
$\mathrm{C} 13-\mathrm{O} 2-\mathrm{H} 1 \mathrm{a}$
$102.66(16)$
C9- $10-\mathrm{C} 11$
$114.56(19)$
$\mathrm{O} 1-\mathrm{H} 1 \mathrm{a}-\mathrm{O} 2$
$160.37(10)$

Hydrogen-bond geometry $\left(\AA,{ }^{\circ}\right)$

\begin{tabular}{lllll}
\hline$D-\mathrm{H} \cdots A$ & $D-\mathrm{H}$ & $\mathrm{H} \cdots A$ & $D \cdots A$ & $D-\mathrm{H} \cdots A$ \\
\hline $\mathrm{C} 4-\mathrm{H} 4 \cdots \mathrm{O} 3^{\mathrm{i}}$ & 0.93 & 2.47 & $3.327(3)$ & 154 \\
$\mathrm{O} 2-\mathrm{H} 1 a \cdots \mathrm{C} 10$ & $1.0386(15)$ & $2.110(2)$ & $2.820(3)$ & $123.5(1)$ \\
$\mathrm{O} 2-\mathrm{H} 1 a \cdots \mathrm{O} 1$ & $1.0386(15)$ & $1.4955(15)$ & $2.498(2)$ & $160.4(1)$ \\
$\mathrm{N} 2-\mathrm{H} 2 a \cdots \mathrm{O} 3$ & $0.967(2)$ & $1.8902(16)$ & $2.838(3)$ & $165.87(12)$ \\
$\mathrm{N} 4-\mathrm{H} 4 a \cdots \mathrm{O} 1^{\mathrm{ii}}$ & $0.9427(18)$ & $1.9236(14)$ & $2.784(2)$ & $150.60(13)$ \\
$\mathrm{N} 4-\mathrm{H} 4 a \cdots 7^{\mathrm{ii}}$ & $0.9427(18)$ & $2.4336(18)$ & $2.873(3)$ & $108.36(13)$
\end{tabular}

Symmetry codes: (i) $x,-y+1, z+1 / 2$; (ii) $-x+3 / 2, y+1 / 2,-z+3 / 2$.

3,5-Dinitrosalicylic acid-phenazine (1/1) (LUDFUL)

\section{Crystal data}

$\mathrm{C}_{7} \mathrm{H}_{4} \mathrm{~N}_{2} \mathrm{O}_{7} \cdot \mathrm{C}_{12} \mathrm{H}_{8} \mathrm{~N}_{2}$

$M_{r}=408.33$

Monoclinic, $P 2{ }_{1} / a$

$a=14.8002(15) \AA$

$b=7.4029(16) \AA$

$c=16.0091(16) \AA$

$\beta=96.395(8)^{\circ}$

$V=1743.1(5) \AA^{3}$

$Z=4$

$F(000)=840$

Data collection

Enraf-Nonius CAD-4 diffractometer

Radiation source: fine-focus sealed tube

Graphite monochromator

w scans

8396 measured reflections

4202 independent reflections

1587 reflections with $I>3 \sigma(I)$

\section{Refinement}

Refinement on $F^{2}$

Least-squares matrix: full

$R[F>3 \sigma(F)]=0.044$

$w R(F)=0.083$

$S=1.08$

4202 reflections

274 parameters

0 restraints

40 constraints

Primary atom site location: structure-invariant direct methods
$D_{\mathrm{x}}=1.556 \mathrm{Mg} \mathrm{m}^{-3}$

Melting point: $471 \mathrm{~K}$

Mo $K \alpha$ radiation, $\lambda=0.71073 \AA$

Cell parameters from 25 reflections

$\theta=5-12^{\circ}$

$\mu=0.12 \mathrm{~mm}^{-1}$

$T=293 \mathrm{~K}$

Rhombic, yellow

$0.36 \times 0.34 \times 0.26 \mathrm{~mm}$

$R_{\text {int }}=0.056$

$\theta_{\max }=28.0^{\circ}, \theta_{\min }=1.5^{\circ}$

$h=0 \rightarrow 19$

$k=-9 \rightarrow 9$

$l=-21 \rightarrow 21$

3 standard reflections every 150 reflections intensity decay: $2 \%$

Secondary atom site location: difference Fourier map

Hydrogen site location: difference Fourier map

$\mathrm{H}$ atoms treated by a mixture of independent and constrained refinement

Weighting scheme based on measured s.u.'s $w=$ $1 /\left(\sigma^{2}(I)+0.0004 I^{2}\right)$

$(\Delta / \sigma)_{\max }=0.006$

$\Delta \rho_{\max }=0.29 \mathrm{e} \AA^{-3}$

$\Delta \rho_{\min }=-0.31 \mathrm{e} \AA^{-3}$

Extinction correction: B-C type 1 Lorentzian isotropic (Becker \& Coppens, 1974)

Extinction coefficient: 5100 (500) 


\section{Special details}

Geometry. All esds (except the esd in the dihedral angle between two 1.s. planes) are estimated using the full covariance matrix. The cell esds are taken into account individually in the estimation of esds in distances, angles and torsion angles; correlations between esds in cell parameters are only used when they are defined by crystal symmetry. An approximate (isotropic) treatment of cell esds is used for estimating esds involving l.s. planes.

Refinement. Refinement of $\mathrm{F}^{2}$ against ALL reflections. The weighted R-factor $\mathrm{wR}$ and goodness of fit $\mathrm{S}$ are based on $\mathrm{F}^{2}$, conventional $R$-factors $R$ are based on $F$, with $F$ set to zero for negative $F^{2}$. The threshold expression of $F^{2}>2 \operatorname{sigma}\left(\mathrm{F}^{2}\right)$ is used only for calculating R-factors(gt) etc. and is not relevant to the choice of reflections for refinement. R-factors based on $\mathrm{F}^{2}$ are statistically about twice as large as those based on F, and R- factors based on ALL data will be even larger.

Number of fixed parameters: 6

Fractional atomic coordinates and isotropic or equivalent isotropic displacement parameters $\left(\AA^{2}\right)$

\begin{tabular}{|c|c|c|c|c|}
\hline & $x$ & $y$ & $z$ & $U_{\text {iso }} * / U_{\text {eq }}$ \\
\hline $\mathrm{O} 3$ & $0.98761(10)$ & $0.2874(2)$ & $0.46747(9)$ & $0.0599(6)$ \\
\hline $\mathrm{O} 1$ & $0.85079(8)$ & $0.4309(2)$ & $0.24121(9)$ & $0.0539(6)$ \\
\hline $\mathrm{C} 1$ & $0.98636(12)$ & $0.4161(3)$ & $0.33159(12)$ & $0.0373(7)$ \\
\hline $\mathrm{O} 7$ & $1.12739(10)$ & $0.6537(3)$ & $0.15665(10)$ & $0.0716(7)$ \\
\hline $\mathrm{C} 4$ & $1.17200(13)$ & $0.4785(3)$ & $0.36328(13)$ & $0.0421(8)$ \\
\hline $\mathrm{H} 4 \mathrm{a}$ & 1.234071 & 0.501018 & 0.373432 & $0.0506^{*}$ \\
\hline $\mathrm{C} 5$ & $1.12429(13)$ & $0.5256(3)$ & $0.28746(12)$ & $0.0378(7)$ \\
\hline $\mathrm{O} 2$ & $0.84456(9)$ & $0.3110(2)$ & $0.36758(9)$ & $0.0632(7)$ \\
\hline $\mathrm{C} 2$ & $1.03254(14)$ & $0.3661(3)$ & $0.40995(13)$ & $0.0430(8)$ \\
\hline $\mathrm{C} 3$ & 1.12654 (13) & $0.3980(3)$ & $0.42348(12)$ & $0.0423(8)$ \\
\hline N1 & $1.18089(15)$ & $0.3455(3)$ & $0.50174(12)$ & $0.0665(9)$ \\
\hline $\mathrm{C} 7$ & $0.88704(14)$ & $0.3818(3)$ & $0.31414(14)$ & 0.0457 (9) \\
\hline C6 & $1.03232(13)$ & $0.4947(3)$ & $0.27113(12)$ & $0.0386(7)$ \\
\hline H6a & 1.001506 & 0.526882 & 0.219465 & $0.0463 *$ \\
\hline O6 & $1.25212(10)$ & $0.6448(3)$ & $0.23814(10)$ & $0.0804(8)$ \\
\hline $\mathrm{O} 4$ & $1.14445(14)$ & $0.2742(3)$ & $0.55587(13)$ & $0.1291(12)$ \\
\hline N2 & $1.17129(12)$ & $0.6128(3)$ & $0.22290(12)$ & $0.0504(8)$ \\
\hline O5 & $1.26041(12)$ & $0.3839(3)$ & $0.51002(10)$ & $0.0996(10)$ \\
\hline N3 & $0.68389(10)$ & $0.3729(2)$ & $0.18982(10)$ & $0.0388(6)$ \\
\hline N4 & 0.50803 & $0.3575(3)$ & $0.10509(11)$ & $0.0517(7)$ \\
\hline $\mathrm{C} 17$ & $0.57960(14)$ & $0.2988(3)$ & $0.06927(13)$ & $0.0505(9)$ \\
\hline C19 & $0.61248(13)$ & $0.4326(3)$ & $0.22726(12)$ & 0.0377 (7) \\
\hline $\mathrm{C} 8$ & $0.74378(15)$ & $0.2489(3)$ & $0.06912(15)$ & $0.0552(9)$ \\
\hline $\mathrm{H} 8 \mathrm{a}$ & 0.802687 & 0.252957 & 0.096215 & $0.0663 *$ \\
\hline $\mathrm{C} 16$ & $0.66987(13)$ & $0.3064(3)$ & $0.11118(13)$ & $0.0405(8)$ \\
\hline $\mathrm{C} 18$ & $0.52369(13)$ & $0.4267(3)$ & $0.18296(13)$ & $0.0413(8)$ \\
\hline $\mathrm{C} 12$ & $0.45074(14)$ & $0.4976(3)$ & $0.22251(15)$ & $0.0533(9)$ \\
\hline $\mathrm{H} 12 \mathrm{a}$ & 0.392387 & 0.498106 & 0.194132 & $0.064^{*}$ \\
\hline $\mathrm{C} 14$ & $0.55304(15)$ & $0.5655(3)$ & $0.34545(14)$ & $0.0522(9)$ \\
\hline $\mathrm{H} 14 \mathrm{a}$ & 0.561295 & 0.610201 & 0.400052 & $0.0627^{*}$ \\
\hline $\mathrm{C} 13$ & $0.46523(15)$ & $0.5645(3)$ & $0.30108(15)$ & $0.0555(10)$ \\
\hline H13a & 0.416606 & 0.610633 & 0.326453 & $0.0666^{*}$ \\
\hline $\mathrm{C} 15$ & $0.62592(14)$ & $0.5020(3)$ & $0.30951(13)$ & $0.0442(8)$ \\
\hline H15a & 0.683759 & 0.504497 & 0.338918 & $0.053 *$ \\
\hline
\end{tabular}




$\begin{array}{lllll}\text { C10 } & 0.6400(2) & 0.1763(4) & -0.05197(16) & 0.0789(12) \\ \text { H10a } & 0.631177 & 0.131533 & -0.10654 & 0.0946^{*} \\ \text { C11 } & 0.56781(17) & 0.2291(3) & -0.01395(15) & 0.0689(11) \\ \text { H11a } & 0.509671 & 0.219906 & -0.042373 & 0.0827^{*} \\ \text { C9 } & 0.72833(18) & 0.1879(3) & -0.01060(16) & 0.0685(12) \\ \text { H9a } & 0.777245 & 0.152877 & -0.038619 & 0.0822^{*} \\ \text { H3a } & 0.919191 & 0.279002 & 0.440153 & 0.138(12)^{*} \\ \text { H1a } & 0.773139 & 0.402666 & 0.229166 & 0.105(8)^{*}\end{array}$

Atomic displacement parameters $\left(\AA^{2}\right)$

\begin{tabular}{|c|c|c|c|c|c|c|}
\hline & $U^{11}$ & $U^{22}$ & $U^{33}$ & $U^{12}$ & $U^{13}$ & $U^{23}$ \\
\hline $\mathrm{O} 3$ & $0.0634(10)$ & $0.0734(13)$ & $0.0442(9)$ & $-0.0001(9)$ & $0.0122(8)$ & $0.0094(9)$ \\
\hline $\mathrm{O} 1$ & $0.0315(8)$ & $0.0749(13)$ & $0.0545(10)$ & $-0.0027(8)$ & $0.0009(7)$ & $0.0096(9)$ \\
\hline $\mathrm{C} 1$ & $0.0315(11)$ & $0.0413(14)$ & $0.0394(12)$ & $0.0004(10)$ & $0.0046(10)$ & $-0.0033(11)$ \\
\hline $\mathrm{O} 7$ & $0.0556(10)$ & $0.1066(17)$ & $0.0520(10)$ & $-0.0111(10)$ & $0.0040(8)$ & $0.0199(11)$ \\
\hline $\mathrm{C} 4$ & $0.0326(11)$ & $0.0468(15)$ & $0.0457(13)$ & $0.0043(11)$ & $-0.0016(11)$ & $-0.0111(12)$ \\
\hline $\mathrm{C} 5$ & $0.0325(11)$ & $0.0414(15)$ & $0.0398(12)$ & $0.0028(10)$ & $0.0059(10)$ & $-0.0035(11)$ \\
\hline $\mathrm{O} 2$ & $0.0445(9)$ & $0.0836(14)$ & $0.0637(10)$ & $-0.0069(9)$ & $0.0161(8)$ & $0.0161(10)$ \\
\hline $\mathrm{C} 2$ & $0.0493(13)$ & $0.0426(15)$ & $0.0377(12)$ & $0.0029(12)$ & $0.0073(11)$ & $-0.0033(12)$ \\
\hline $\mathrm{C} 3$ & $0.0449(12)$ & $0.0468(16)$ & $0.0337(12)$ & $0.0128(11)$ & $-0.0024(10)$ & $-0.0056(12)$ \\
\hline N1 & $0.0646(15)$ & $0.090(2)$ & $0.0420(13)$ & $0.0141(14)$ & $-0.0078(12)$ & $-0.0014(13)$ \\
\hline $\mathrm{C} 7$ & $0.0413(13)$ & $0.0461(16)$ & $0.0509(14)$ & $-0.0004(12)$ & $0.0098(11)$ & $-0.0019(13)$ \\
\hline C6 & $0.0334(11)$ & $0.0433(14)$ & $0.0381(12)$ & $0.0051(10)$ & $-0.0003(10)$ & $-0.0035(11)$ \\
\hline O6 & $0.0328(8)$ & $0.1225(17)$ & $0.0871(12)$ & $-0.0130(10)$ & $0.0128(8)$ & $0.0119(12)$ \\
\hline $\mathrm{O} 4$ & $0.1041(16)$ & $0.197(3)$ & $0.0807(15)$ & $-0.0108(16)$ & $-0.0151(13)$ & $0.0758(17)$ \\
\hline $\mathrm{N} 2$ & $0.0369(11)$ & $0.0590(15)$ & $0.0567(13)$ & $-0.0011(11)$ & $0.0116(10)$ & $-0.0046(12)$ \\
\hline O5 & $0.0575(11)$ & $0.177(2)$ & $0.0581(11)$ & $0.0108(14)$ & $-0.0193(9)$ & $-0.0056(13)$ \\
\hline N3 & $0.0310(9)$ & $0.0431(12)$ & $0.0419(10)$ & $-0.0041(9)$ & $0.0024(8)$ & $0.0009(10)$ \\
\hline N4 & $0.0446(11)$ & $0.0533(14)$ & $0.0545(12)$ & $0.0017(10)$ & $-0.0065(9)$ & $-0.0035(11)$ \\
\hline $\mathrm{C} 17$ & $0.0538(14)$ & $0.0491(17)$ & $0.0469(14)$ & $0.0046(13)$ & $-0.0024(12)$ & $-0.0024(13)$ \\
\hline C19 & $0.0354(12)$ & $0.0362(14)$ & $0.0412(13)$ & $-0.0040(11)$ & $0.0029(10)$ & $0.0023(11)$ \\
\hline $\mathrm{C} 8$ & $0.0564(15)$ & $0.0521(18)$ & $0.0588(16)$ & $0.0030(13)$ & $0.0136(13)$ & $-0.0013(13)$ \\
\hline $\mathrm{C} 16$ & $0.0459(13)$ & $0.0339(14)$ & $0.0419(13)$ & $-0.0006(11)$ & $0.0057(11)$ & $0.0018(11)$ \\
\hline $\mathrm{C} 18$ & $0.0352(11)$ & $0.0378(15)$ & $0.0504(13)$ & $-0.0009(11)$ & $0.0032(10)$ & $-0.0008(12)$ \\
\hline $\mathrm{C} 12$ & $0.0355(13)$ & $0.0510(17)$ & $0.0730(17)$ & $0.0020(12)$ & $0.0041(12)$ & $-0.0052(14)$ \\
\hline $\mathrm{C} 14$ & $0.0630(15)$ & $0.0471(17)$ & $0.0484(14)$ & $-0.0037(14)$ & $0.0143(13)$ & $-0.0049(13)$ \\
\hline $\mathrm{C} 13$ & $0.0457(14)$ & $0.0512(18)$ & $0.0728(18)$ & $0.0032(13)$ & $0.0208(13)$ & $-0.0040(15)$ \\
\hline $\mathrm{C} 15$ & $0.0419(13)$ & $0.0481(16)$ & $0.0418(13)$ & $-0.0029(12)$ & $0.0012(11)$ & $0.0047(12)$ \\
\hline $\mathrm{C} 10$ & $0.107(2)$ & $0.078(2)$ & $0.0504(16)$ & $0.009(2)$ & $0.0051(17)$ & $-0.0198(16)$ \\
\hline $\mathrm{C} 11$ & $0.0775(19)$ & $0.071(2)$ & $0.0533(17)$ & $0.0057(16)$ & $-0.0147(14)$ & $-0.0148(15)$ \\
\hline C9 & $0.089(2)$ & $0.060(2)$ & $0.0602(18)$ & $0.0060(17)$ & $0.0270(15)$ & $-0.0069(16)$ \\
\hline
\end{tabular}

Geometric parameters $\left(\AA,{ }^{\circ}\right)$

\begin{tabular}{llll}
\hline $\mathrm{O} 3-\mathrm{C} 2$ & $1.329(3)$ & $\mathrm{N} 4-\mathrm{C} 17$ & $1.332(3)$ \\
$\mathrm{O} 3-\mathrm{H} 3 \mathrm{a}$ & $1.0592(14)$ & $\mathrm{N} 4-\mathrm{C} 18$ & $1.344(3)$ \\
$\mathrm{O} 1-\mathrm{C} 7$ & $1.282(3)$ & $\mathrm{C} 17-\mathrm{C} 16$ & $1.428(3)$
\end{tabular}




\begin{tabular}{|c|c|c|c|}
\hline $\mathrm{O} 1-\mathrm{H} 1 \mathrm{a}$ & $1.1628(13)$ & $\mathrm{C} 17-\mathrm{C} 11$ & $1.421(3)$ \\
\hline $\mathrm{C} 1-\mathrm{C} 2$ & $1.410(3)$ & $\mathrm{C} 19-\mathrm{C} 18$ & $1.423(3)$ \\
\hline $\mathrm{C} 1-\mathrm{C} 7$ & $1.487(3)$ & $\mathrm{C} 19-\mathrm{C} 15$ & $1.407(3)$ \\
\hline $\mathrm{C} 1-\mathrm{C} 6$ & $1.373(3)$ & $\mathrm{C} 8-\mathrm{H} 8 \mathrm{a}$ & 0.93 \\
\hline $\mathrm{O} 7-\mathrm{N} 2$ & $1.219(2)$ & $\mathrm{C} 8-\mathrm{C} 16$ & $1.413(3)$ \\
\hline $\mathrm{C} 4-\mathrm{H} 4 \mathrm{a}$ & 0.93 & $\mathrm{C} 8-\mathrm{C} 9$ & $1.349(3)$ \\
\hline $\mathrm{C} 4-\mathrm{C} 5$ & $1.379(3)$ & $\mathrm{C} 18-\mathrm{C} 12$ & $1.412(3)$ \\
\hline $\mathrm{C} 4-\mathrm{C} 3$ & $1.371(3)$ & $\mathrm{C} 12-\mathrm{H} 12 \mathrm{a}$ & 0.93 \\
\hline $\mathrm{C} 5-\mathrm{C} 6$ & $1.376(3)$ & $\mathrm{C} 12-\mathrm{C} 13$ & $1.347(3)$ \\
\hline $\mathrm{C} 5-\mathrm{N} 2$ & $1.459(3)$ & $\mathrm{C} 14-\mathrm{H} 14 \mathrm{a}$ & 0.93 \\
\hline $\mathrm{O} 2-\mathrm{C} 7$ & $1.234(3)$ & $\mathrm{C} 14-\mathrm{C} 13$ & $1.410(3)$ \\
\hline $\mathrm{C} 2-\mathrm{C} 3$ & $1.404(3)$ & $\mathrm{C} 14-\mathrm{C} 15$ & $1.361(3)$ \\
\hline $\mathrm{C} 3-\mathrm{N} 1$ & $1.464(3)$ & $\mathrm{C} 13-\mathrm{H} 13 \mathrm{a}$ & 0.93 \\
\hline $\mathrm{N} 1-\mathrm{O} 4$ & $1.194(3)$ & $\mathrm{C} 15-\mathrm{H} 15 \mathrm{a}$ & 0.93 \\
\hline $\mathrm{N} 1-\mathrm{O} 5$ & $1.204(3)$ & $\mathrm{C} 10-\mathrm{H} 10 \mathrm{a}$ & 0.93 \\
\hline C6-H6a & 0.93 & $\mathrm{C} 10-\mathrm{C} 11$ & $1.346(4)$ \\
\hline $\mathrm{O} 6-\mathrm{N} 2$ & $1.217(2)$ & $\mathrm{C} 10-\mathrm{C} 9$ & $1.400(4)$ \\
\hline N3-C19 & $1.346(3)$ & $\mathrm{C} 11-\mathrm{H} 11 \mathrm{a}$ & 0.93 \\
\hline $\mathrm{N} 3-\mathrm{C} 16$ & $1.346(3)$ & $\mathrm{C} 9-\mathrm{H} 9 \mathrm{a}$ & 0.93 \\
\hline $\mathrm{C} 2-\mathrm{O} 3-\mathrm{H} 3 \mathrm{a}$ & $105.60(13)$ & $\mathrm{C} 16-\mathrm{C} 17-\mathrm{C} 11$ & $117.8(2)$ \\
\hline $\mathrm{C} 7-\mathrm{O} 1-\mathrm{H} 1 \mathrm{a}$ & $113.94(15)$ & $\mathrm{N} 3-\mathrm{C} 19-\mathrm{C} 18$ & $119.68(18)$ \\
\hline $\mathrm{C} 2-\mathrm{C} 1-\mathrm{C} 7$ & $119.58(18)$ & $\mathrm{N} 3-\mathrm{C} 19-\mathrm{C} 15$ & $120.03(17)$ \\
\hline $\mathrm{C} 2-\mathrm{C} 1-\mathrm{C} 6$ & $120.63(17)$ & $\mathrm{C} 18-\mathrm{C} 19-\mathrm{C} 15$ & $120.28(19)$ \\
\hline $\mathrm{C} 7-\mathrm{C} 1-\mathrm{C} 6$ & $119.79(17)$ & $\mathrm{H} 8 \mathrm{a}-\mathrm{C} 8-\mathrm{C} 16$ & 120.23 \\
\hline $\mathrm{H} 4 \mathrm{a}-\mathrm{C} 4-\mathrm{C} 5$ & 120.49 & $\mathrm{H} 8 \mathrm{a}-\mathrm{C} 8-\mathrm{C} 9$ & 120.23 \\
\hline $\mathrm{H} 4 \mathrm{a}-\mathrm{C} 4-\mathrm{C} 3$ & 120.49 & $\mathrm{C} 16-\mathrm{C} 8-\mathrm{C} 9$ & $119.5(2)$ \\
\hline $\mathrm{C} 5-\mathrm{C} 4-\mathrm{C} 3$ & $119.01(18)$ & $\mathrm{N} 3-\mathrm{C} 16-\mathrm{C} 17$ & $119.52(18)$ \\
\hline $\mathrm{C} 4-\mathrm{C} 5-\mathrm{C} 6$ & $121.58(19)$ & $\mathrm{N} 3-\mathrm{C} 16-\mathrm{C} 8$ & $120.61(17)$ \\
\hline $\mathrm{C} 4-\mathrm{C} 5-\mathrm{N} 2$ & $119.83(17)$ & $\mathrm{C} 17-\mathrm{C} 16-\mathrm{C} 8$ & $119.86(19)$ \\
\hline $\mathrm{C} 6-\mathrm{C} 5-\mathrm{N} 2$ & $118.58(17)$ & $\mathrm{N} 4-\mathrm{C} 18-\mathrm{C} 19$ & $121.90(19)$ \\
\hline $\mathrm{C} 7-\mathrm{O} 2-\mathrm{H} 3 \mathrm{a}$ & $102.24(13)$ & $\mathrm{N} 4-\mathrm{C} 18-\mathrm{C} 12$ & $119.74(18)$ \\
\hline $\mathrm{O} 3-\mathrm{C} 2-\mathrm{C} 1$ & $120.09(17)$ & $\mathrm{C} 19-\mathrm{C} 18-\mathrm{C} 12$ & $118.35(19)$ \\
\hline $\mathrm{O} 3-\mathrm{C} 2-\mathrm{C} 3$ & $122.05(17)$ & $\mathrm{C} 18-\mathrm{C} 12-\mathrm{H} 12 \mathrm{a}$ & 119.82 \\
\hline $\mathrm{C} 1-\mathrm{C} 2-\mathrm{C} 3$ & $117.84(19)$ & $\mathrm{C} 18-\mathrm{C} 12-\mathrm{C} 13$ & $120.35(19)$ \\
\hline $\mathrm{C} 4-\mathrm{C} 3-\mathrm{C} 2$ & $121.34(18)$ & $\mathrm{H} 12 \mathrm{a}-\mathrm{C} 12-\mathrm{C} 13$ & 119.82 \\
\hline $\mathrm{C} 4-\mathrm{C} 3-\mathrm{N} 1$ & $116.79(18)$ & $\mathrm{H} 14 \mathrm{a}-\mathrm{C} 14-\mathrm{C} 13$ & 119.52 \\
\hline $\mathrm{C} 2-\mathrm{C} 3-\mathrm{N} 1$ & $121.86(19)$ & $\mathrm{H} 14 \mathrm{a}-\mathrm{C} 14-\mathrm{C} 15$ & 119.52 \\
\hline $\mathrm{C} 3-\mathrm{N} 1-\mathrm{O} 4$ & $119.3(2)$ & $\mathrm{C} 13-\mathrm{C} 14-\mathrm{C} 15$ & $121.0(2)$ \\
\hline $\mathrm{C} 3-\mathrm{N} 1-\mathrm{O} 5$ & $118.0(2)$ & $\mathrm{C} 12-\mathrm{C} 13-\mathrm{C} 14$ & $121.0(2)$ \\
\hline $\mathrm{O} 4-\mathrm{N} 1-\mathrm{O} 5$ & $122.7(2)$ & $\mathrm{C} 12-\mathrm{C} 13-\mathrm{H} 13 \mathrm{a}$ & 119.52 \\
\hline $\mathrm{O} 1-\mathrm{C} 7-\mathrm{C} 1$ & $115.30(19)$ & $\mathrm{C} 14-\mathrm{C} 13-\mathrm{H} 13 \mathrm{a}$ & 119.52 \\
\hline $\mathrm{O} 1-\mathrm{C} 7-\mathrm{O} 2$ & $123.93(18)$ & $\mathrm{C} 19-\mathrm{C} 15-\mathrm{C} 14$ & $119.05(18)$ \\
\hline $\mathrm{C} 1-\mathrm{C} 7-\mathrm{O} 2$ & $120.76(18)$ & $\mathrm{C} 19-\mathrm{C} 15-\mathrm{H} 15 \mathrm{a}$ & 120.48 \\
\hline $\mathrm{C} 1-\mathrm{C} 6-\mathrm{C} 5$ & $119.59(17)$ & $\mathrm{C} 14-\mathrm{C} 15-\mathrm{H} 15 \mathrm{a}$ & 120.48 \\
\hline $\mathrm{C} 1-\mathrm{C} 6-\mathrm{H} 6 \mathrm{a}$ & 120.21 & $\mathrm{H} 10 \mathrm{a}-\mathrm{C} 10-\mathrm{C} 11$ & 119.51 \\
\hline $\mathrm{C} 5-\mathrm{C} 6-\mathrm{H} 6 \mathrm{a}$ & 120.2 & $\mathrm{H} 10 \mathrm{a}-\mathrm{C} 10-\mathrm{C} 9$ & 119.51 \\
\hline $\mathrm{O} 7-\mathrm{N} 2-\mathrm{C} 5$ & $118.45(16)$ & $\mathrm{C} 11-\mathrm{C} 10-\mathrm{C} 9$ & $121.0(2)$ \\
\hline
\end{tabular}




$\begin{array}{llll}\mathrm{O} 7-\mathrm{N} 2-\mathrm{O} 6 & 122.87(19) & \mathrm{C} 17-\mathrm{C} 11-\mathrm{C} 10 & 120.6(2) \\ \mathrm{C} 5-\mathrm{N} 2-\mathrm{O} 6 & 118.66(17) & \mathrm{C} 17-\mathrm{C} 11-\mathrm{H} 11 \mathrm{a} & 119.71 \\ \mathrm{C} 19-\mathrm{N} 3-\mathrm{C} 16 & 119.31(15) & \mathrm{C} 10-\mathrm{C} 11-\mathrm{H} 11 \mathrm{a} & 119.71 \\ \mathrm{C} 19-\mathrm{N} 3-\mathrm{H} 1 \mathrm{a} & 119.38(14) & \mathrm{C} 8-\mathrm{C} 9-\mathrm{C} 10 & 121.3(3) \\ \mathrm{C} 16-\mathrm{N} 3-\mathrm{H} 1 \mathrm{a} & 120.79(14) & \mathrm{C} 8-\mathrm{C} 9-\mathrm{H} 9 \mathrm{a} & 119.37 \\ \mathrm{C} 17-\mathrm{N} 4-\mathrm{C} 18 & 117.41(17) & \mathrm{C} 10-\mathrm{C} 9-\mathrm{H} 9 \mathrm{a} & 119.37 \\ \mathrm{~N} 4-\mathrm{C} 17-\mathrm{C} 16 & 122.14(19) & \mathrm{O} 3-\mathrm{H} 3 \mathrm{a}-\mathrm{O} 2 & 151.72(10) \\ \mathrm{N} 4-\mathrm{C} 17-\mathrm{C} 11 & 120.09(19) & \mathrm{O} 1-\mathrm{H} 1 \mathrm{a}-\mathrm{N} 3 & 163.24(10)\end{array}$

Hydrogen-bond geometry $\left(\AA,{ }^{\circ}\right)$

\begin{tabular}{lllll}
\hline$D-\mathrm{H} \cdots A$ & $D-\mathrm{H}$ & $\mathrm{H} \cdots A$ & $D \cdots A$ & $D-\mathrm{H} \cdots A$ \\
\hline $\mathrm{C} 13-\mathrm{H} 13 a \cdots \mathrm{O} 44^{\mathrm{i}}$ & 0.93 & 2.49 & $3.334(3)$ & 151 \\
$\mathrm{O} 3-\mathrm{H} 3 a \cdots \mathrm{O} 2$ & $1.0592(14)$ & $1.5297(14)$ & $2.5132(19)$ & $151.72(10)$ \\
$\mathrm{O} 1-\mathrm{H} 1 a \cdots \mathrm{N} 3$ & $1.1628(13)$ & $1.4160(14)$ & $2.5515(19)$ & $163.24(10)$ \\
\hline
\end{tabular}

Symmetry code: (i) $-x+3 / 2, y+1 / 2,-z+1$.

2-Amino-5-methylpyridinium 2-hydroxy-3,5-dinitrobenzoate (NUQVEB)

Crystal data

$\mathrm{C}_{6} \mathrm{H}_{9} \mathrm{~N}_{2}^{+} \cdot \mathrm{C}_{7} \mathrm{H}_{3} \mathrm{~N}_{2} \mathrm{O}_{7}^{-}$

$M_{r}=336.27$

Triclinic, $P \overline{1}$

Hall symbol: -P 1

$a=5.8673(7) \AA$

$b=8.0991(9) \AA$

$c=15.2437(17) \AA$

$\alpha=86.844(3)^{\circ}$

$\beta=84.252(3)^{\circ}$

$\gamma=81.209(3)^{\circ}$

$V=711.69(14) \AA^{3}$

Data collection

Bruker APEX DUO CCD area-detector diffractometer

Radiation source: fine-focus sealed tube

Graphite monochromator

$\varphi$ and $\omega$ scans

Absorption correction: multi-scan

(SADABS; Bruker, 2009)

$T_{\min }=0.963, T_{\max }=0.990$

Refinement

Refinement on $F^{2}$

Least-squares matrix: full

$R[F>3 \sigma(F)]=0.042$

$w R(F)=0.109$

$S=2.06$

4943 reflections

222 parameters

0 restraints

34 constraints
$Z=2$

$F(000)=348$

$D_{\mathrm{x}}=1.569 \mathrm{Mg} \mathrm{m}^{-3}$

Mo $K \alpha$ radiation, $\lambda=0.71073 \AA$

Cell parameters from 5139 reflections

$\theta=2.7-32.4^{\circ}$

$\mu=0.13 \mathrm{~mm}^{-1}$

$T=100 \mathrm{~K}$

Block, yellow

$0.29 \times 0.14 \times 0.08 \mathrm{~mm}$

12709 measured reflections

4943 independent reflections

3677 reflections with $I>3 \sigma(I)$

$R_{\text {int }}=0.023$

$\theta_{\max }=32.5^{\circ}, \theta_{\min }=1.3^{\circ}$

$h=-8 \rightarrow 8$

$k=-12 \rightarrow 11$

$l=-22 \rightarrow 23$

Primary atom site location: structure-invariant direct methods

Secondary atom site location: difference Fourier map

Hydrogen site location: difference Fourier map

$\mathrm{H}$ atoms treated by a mixture of independent and constrained refinement

Weighting scheme based on measured s.u.'s $w=$ $1 /\left(\sigma^{2}(I)+0.0004 I^{2}\right)$ 
$(\Delta / \sigma)_{\max }=0.009$

$\Delta \rho_{\min }=-0.32$ e $\AA^{-3}$

$\Delta \rho_{\max }=0.40 \mathrm{e} \AA^{-3}$

Special details

Experimental. The crystal was placed in the cold stream of an Oxford Cryosystems Cobra open-flow nitrogen cryostat (Cosier \& Glazer, 1986) operating at 100.0 (1) K.

Geometry. All s.u.'s (except the s.u. in the dihedral angle between two 1.s. planes) are estimated using the full covariance matrix. The cell s.u.'s are taken into account individually in the estimation of s.u.'s in distances, angles and torsion angles; correlations between s.u.'s in cell parameters are only used when they are defined by crystal symmetry. An approximate (isotropic) treatment of cell s.u.'s is used for estimating s.u.'s involving l.s. planes.

Refinement. Refinement of $\mathrm{F}^{2}$ against ALL reflections. The weighted R-factor $\mathrm{wR}$ and goodness of fit $\mathrm{S}$ are based on $\mathrm{F}^{2}$, conventional $\mathrm{R}$-factors $\mathrm{R}$ are based on $\mathrm{F}$, with $\mathrm{F}$ set to zero for negative $\mathrm{F}^{2}$. The threshold expression of $\mathrm{F}^{2}>2 \sigma\left(\mathrm{F}^{2}\right)$ is used only for calculating R-factors(gt) etc. and is not relevant to the choice of reflections for refinement. R-factors based on $\mathrm{F}^{2}$ are statistically about twice as large as those based on F, and R- factors based on ALL data will be even larger.

Number of fixed parameters: 15

Fractional atomic coordinates and isotropic or equivalent isotropic displacement parameters $\left(\AA^{2}\right)$

\begin{tabular}{|c|c|c|c|c|c|}
\hline & $x$ & $y$ & $z$ & $U_{\text {iso }} * / U_{\text {eq }}$ & Occ. $(<1)$ \\
\hline N1 & $0.50355(15)$ & $0.28624(11)$ & $0.24318(6)$ & $0.0153(3)$ & \\
\hline $\mathrm{N} 2$ & $0.82331(16)$ & $0.41649(12)$ & $0.19964(6)$ & $0.0182(3)$ & \\
\hline $\mathrm{C} 1$ & $0.67739(18)$ & $0.36376(13)$ & $0.26429(7)$ & $0.0152(3)$ & \\
\hline $\mathrm{C} 2$ & $0.35124(18)$ & $0.22197(14)$ & $0.30471(7)$ & $0.0165(3)$ & \\
\hline $\mathrm{H} 2$ & 0.233313 & 0.167725 & 0.285731 & $0.0198^{*}$ & \\
\hline $\mathrm{C} 3$ & $0.36539(18)$ & $0.23424(14)$ & $0.39257(7)$ & $0.0180(3)$ & \\
\hline $\mathrm{C} 4$ & $0.54266(19)$ & $0.31918(15)$ & $0.41642(7)$ & $0.0200(3)$ & \\
\hline $\mathrm{H} 4$ & 0.556083 & 0.332079 & 0.477169 & $0.024 *$ & \\
\hline $\mathrm{C} 5$ & $0.69461(19)$ & $0.38301(14)$ & $0.35491(7)$ & $0.0186(3)$ & \\
\hline H5 & 0.811136 & 0.439959 & 0.372833 & $0.0223^{*}$ & \\
\hline C6 & $0.2014(2)$ & $0.16274(17)$ & $0.46143(8)$ & $0.0262(4)$ & \\
\hline H6a & 0.118019 & 0.252439 & 0.498084 & $0.0394 *$ & \\
\hline $\mathrm{H} 6 \mathrm{~b}$ & 0.090144 & 0.110743 & 0.432458 & $0.0394 *$ & \\
\hline H6c & 0.289083 & 0.078457 & 0.498608 & $0.0394 *$ & \\
\hline $\mathrm{O} 1$ & $0.17284(13)$ & $0.61818(10)$ & $0.14277(5)$ & $0.0201(2)$ & \\
\hline $\mathrm{O} 2$ & $0.10549(15)$ & $0.60964(11)$ & $0.31689(5)$ & $0.0252(3)$ & \\
\hline $\mathrm{O} 3$ & $0.28472(15)$ & $0.76461(12)$ & $0.38855(5)$ & $0.0275(3)$ & \\
\hline $\mathrm{O} 4$ & $0.93312(14)$ & $0.99538(11)$ & $0.26216(6)$ & $0.0242(3)$ & \\
\hline O5 & $0.99966(15)$ & $1.02486(11)$ & $0.11988(6)$ & 0.0279 & \\
\hline O6 & $0.55543(14)$ & $0.76370(11)$ & $-0.07523(5)$ & $0.0224(3)$ & \\
\hline O7 & $0.26607(14)$ & $0.63258(10)$ & $-0.01549(5)$ & $0.0199(2)$ & \\
\hline N3 & $0.25052(15)$ & $0.70323(12)$ & $0.31961(6)$ & $0.0172(3)$ & \\
\hline N4 & $0.89095(16)$ & $0.97282(12)$ & $0.18597(6)$ & $0.0190(3)$ & \\
\hline $\mathrm{C} 7$ & $0.33912(17)$ & $0.70052(13)$ & $0.15501(7)$ & $0.0141(3)$ & \\
\hline $\mathrm{C} 8$ & $0.38900(17)$ & $0.74506(13)$ & $0.23906(7)$ & $0.0144(3)$ & \\
\hline C9 & $0.56997(17)$ & $0.83259(13)$ & $0.24874(7)$ & $0.0156(3)$ & \\
\hline H9 & 0.601439 & 0.859704 & 0.305724 & $0.0187^{*}$ & \\
\hline $\mathrm{C} 10$ & $0.70326(17)$ & $0.87968(13)$ & $0.17493(7)$ & $0.0154(3)$ & \\
\hline $\mathrm{C} 11$ & $0.66121(18)$ & $0.84357(13)$ & $0.09021(7)$ & $0.0157(3)$ & \\
\hline H11 & 0.753695 & 0.879143 & 0.040052 & $0.0188^{*}$ & \\
\hline
\end{tabular}




\begin{tabular}{llllll}
$\mathrm{C} 12$ & $0.48279(17)$ & $0.75522(13)$ & $0.08058(7)$ & $0.0139(3)$ & \\
$\mathrm{C} 13$ & $0.43623(18)$ & $0.71649(13)$ & $-0.01006(7)$ & $0.0165(3)$ & \\
$\mathrm{H} 1 \mathrm{o} 7$ & 0.20768 & 0.615457 & 0.041923 & $0.044(6)^{*}$ & $0.62(3)$ \\
$\mathrm{H} 1 \mathrm{o} 1$ & 0.186813 & 0.613081 & 0.081569 & $0.044(6)^{*}$ & $0.38(3)$ \\
$\mathrm{H} 2 \mathrm{a}$ & 0.809476 & 0.397973 & 0.143402 & $0.035(4)^{*}$ & \\
$\mathrm{H} 2 \mathrm{~b}$ & 0.928572 & 0.469891 & 0.211657 & $0.048(5)^{*}$ & \\
$\mathrm{H} 1$ & 0.481117 & 0.276133 & 0.186443 & $0.032(4)^{*}$ & \\
\hline
\end{tabular}

Atomic displacement parameters $\left(\AA^{2}\right)$

\begin{tabular}{lllllll}
\hline & $U^{11}$ & $U^{22}$ & $U^{33}$ & $U^{12}$ & $U^{13}$ & $U^{23}$ \\
\hline $\mathrm{N} 1$ & $0.0166(4)$ & $0.0168(5)$ & $0.0130(4)$ & $-0.0032(3)$ & $-0.0023(3)$ & $-0.0006(3)$ \\
$\mathrm{N} 2$ & $0.0194(4)$ & $0.0221(5)$ & $0.0147(4)$ & $-0.0085(4)$ & $-0.0004(3)$ & $-0.0009(4)$ \\
$\mathrm{C} 1$ & $0.0166(5)$ & $0.0137(5)$ & $0.0152(5)$ & $-0.0012(4)$ & $-0.0022(4)$ & $-0.0004(4)$ \\
$\mathrm{C} 2$ & $0.0142(5)$ & $0.0162(5)$ & $0.0192(5)$ & $-0.0026(4)$ & $-0.0015(4)$ & $-0.0007(4)$ \\
$\mathrm{C} 3$ & $0.0182(5)$ & $0.0180(5)$ & $0.0168(5)$ & $-0.0011(4)$ & $0.0004(4)$ & $0.0009(4)$ \\
C4 & $0.0239(5)$ & $0.0239(6)$ & $0.0127(5)$ & $-0.0043(5)$ & $-0.0035(4)$ & $-0.0007(4)$ \\
C5 & $0.0201(5)$ & $0.0201(6)$ & $0.0169(5)$ & $-0.0054(4)$ & $-0.0041(4)$ & $-0.0004(4)$ \\
C6 & $0.0238(6)$ & $0.0324(7)$ & $0.0219(6)$ & $-0.0077(5)$ & $0.0028(4)$ & $0.0047(5)$ \\
O1 & $0.0192(4)$ & $0.0259(4)$ & $0.0176(4)$ & $-0.0108(3)$ & $-0.0027(3)$ & $-0.0010(3)$ \\
O2 & $0.0275(4)$ & $0.0281(5)$ & $0.0220(4)$ & $-0.0141(4)$ & $0.0034(3)$ & $-0.0013(3)$ \\
O3 & $0.0290(5)$ & $0.0434(6)$ & $0.0120(4)$ & $-0.0097(4)$ & $-0.0019(3)$ & $-0.0062(4)$ \\
O4 & $0.0215(4)$ & $0.0234(4)$ & $0.0302(5)$ & $-0.0036(3)$ & $-0.0110(3)$ & $-0.0066(4)$ \\
O5 & $0.0227(4)$ & $0.0276(5)$ & $0.0354(5)$ & $-0.0122(4)$ & $-0.0009(3)$ & $0.0022(4)$ \\
O6 & $0.0280(4)$ & $0.0278(5)$ & $0.0128(4)$ & $-0.0102(4)$ & $0.0001(3)$ & $-0.0006(3)$ \\
O7 & $0.0229(4)$ & $0.0252(4)$ & $0.0139(4)$ & $-0.0096(3)$ & $-0.0034(3)$ & $-0.0010(3)$ \\
N3 & $0.0168(4)$ & $0.0200(5)$ & $0.0145(4)$ & $-0.0020(4)$ & $-0.0017(3)$ & $-0.0002(4)$ \\
N4 & $0.0154(4)$ & $0.0150(5)$ & $0.0275(5)$ & $-0.0027(4)$ & $-0.0051(4)$ & $-0.0025(4)$ \\
C7 & $0.0135(4)$ & $0.0136(5)$ & $0.0150(5)$ & $-0.0010(4)$ & $-0.0022(4)$ & $-0.0010(4)$ \\
C8 & $0.0145(5)$ & $0.0155(5)$ & $0.0128(5)$ & $-0.0016(4)$ & $0.0000(4)$ & $-0.0003(4)$ \\
C9 & $0.0144(5)$ & $0.0156(5)$ & $0.0169(5)$ & $0.0000(4)$ & $-0.0038(4)$ & $-0.0031(4)$ \\
C10 & $0.0128(4)$ & $0.0131(5)$ & $0.0210(5)$ & $-0.0030(4)$ & $-0.0035(4)$ & $-0.0018(4)$ \\
C11 & $0.0148(5)$ & $0.0137(5)$ & $0.0179(5)$ & $-0.0009(4)$ & $-0.0007(4)$ & $-0.0005(4)$ \\
C12 & $0.0145(4)$ & $0.0141(5)$ & $0.0132(5)$ & $-0.0020(4)$ & $-0.0018(4)$ & $-0.0009(4)$ \\
C13 & $0.0190(5)$ & $0.0153(5)$ & $0.0151(5)$ & $-0.0021(4)$ & $-0.0026(4)$ & $-0.0012(4)$ \\
& & & & & & \\
\hline & & & & & &
\end{tabular}

Geometric parameters $\left(\AA,{ }^{\circ}\right)$

\begin{tabular}{llll}
\hline $\mathrm{N} 1-\mathrm{C} 1$ & $1.3498(15)$ & $\mathrm{O} 1-\mathrm{H} 1 \mathrm{o} 1$ & $0.9310(8)$ \\
$\mathrm{N} 1-\mathrm{C} 2$ & $1.3674(14)$ & $\mathrm{O} 2-\mathrm{N} 3$ & $1.2280(14)$ \\
$\mathrm{N} 1-\mathrm{H} 1$ & $0.8977(9)$ & $\mathrm{O} 3-\mathrm{N} 3$ & $1.2338(13)$ \\
$\mathrm{N} 2-\mathrm{C} 1$ & $1.3353(14)$ & $\mathrm{O} 4-\mathrm{N} 4$ & $1.2402(14)$ \\
$\mathrm{N} 2-\mathrm{H} 2 \mathrm{a}$ & $0.8921(9)$ & $\mathrm{O} 5-\mathrm{N} 4$ & $1.2273(13)$ \\
$\mathrm{N} 2-\mathrm{H} 2 \mathrm{~b}$ & $0.8456(10)$ & $\mathrm{O} 6-\mathrm{C} 13$ & $1.2340(13)$ \\
$\mathrm{C} 1-\mathrm{C} 5$ & $1.4139(15)$ & $\mathrm{O} 7-\mathrm{C} 13$ & $1.3022(15)$ \\
$\mathrm{C} 2-\mathrm{H} 2$ & 0.95 & $\mathrm{O} 7-\mathrm{H} 1 \mathrm{o} 7$ & $0.9185(8)$ \\
$\mathrm{C} 2-\mathrm{C} 3$ & $1.3602(16)$ & $\mathrm{N} 3-\mathrm{C} 8$ & $1.4564(13)$ \\
$\mathrm{C} 3-\mathrm{C} 4$ & $1.4174(17)$ & $\mathrm{N} 4-\mathrm{C} 10$ & $1.4544(15)$
\end{tabular}




C3-C6
C4-H4
C4-C5
C5-H5
C6-H6a
C6-H6b
C6-H6c
H6a-H6b
H6a-H6c
H6b-H6c
O1-C7
C1-N1-C2
C1-N1-H1
C2-N1-H1
C1-N2-H2a
C1-N2-H2b
H2a-N2-H2b
N1-C1-N2
N1-C1-C5
N2-C1-C5
N1-C2-H2
N1-C2-C3
H2-C2-C3
C2-C3-C4
C2-C3-C6
C4-C3-C6
C3-C4-H4
C3-C4-C5
H4-C4-C5
C1-C5-C4
C1-C5-H5
C4-C5-H5
C3-C6-H6a
C3-C6-H6b
C3-C6-H6c
H6a-C6-H6b
H6a-C6-H6c
H6b-C6-H6c
C7-O1-H1o7
C7-O1-H1o1
C13-O7-H1o7
C13-O7-H1o1

C3- 66

$\mathrm{C} 5-\mathrm{H} 5$

C6- 6 6a

$\mathrm{C} 6-\mathrm{H} 6 \mathrm{~b}$

C6- $\mathrm{H} 6 \mathrm{c}$

$\mathrm{H} 6 \mathrm{a}-\mathrm{H} 6 \mathrm{c}$

$\mathrm{C} 1-\mathrm{N} 1-\mathrm{C} 2$

$\mathrm{C} 1-\mathrm{N} 1-\mathrm{H} 1$

$\mathrm{C} 1-\mathrm{N} 2-\mathrm{H} 2 \mathrm{a}$

$\mathrm{C} 1-\mathrm{N} 2-\mathrm{H} 2 \mathrm{~b}$

$\mathrm{N} 1-\mathrm{C} 1-\mathrm{N} 2$

$\mathrm{N} 1-\mathrm{C} 1-\mathrm{C} 5$

$\mathrm{N} 2-\mathrm{C} 1-\mathrm{C} 5$

$\mathrm{N} 1-\mathrm{C} 2-\mathrm{H} 2$

$\mathrm{N} 1-\mathrm{C} 2-\mathrm{C} 3$

$\mathrm{H} 2-\mathrm{C} 2-\mathrm{C} 3$

C2- $3-\mathrm{C} 6$

$\mathrm{C} 4-\mathrm{C} 3-\mathrm{C} 6$

$\mathrm{C} 3-\mathrm{C} 4-\mathrm{H} 4$

$\mathrm{C} 1-\mathrm{C} 5-\mathrm{C} 4$

$\mathrm{C} 1-\mathrm{C} 5-\mathrm{H} 5$

$\mathrm{C} 4-\mathrm{C} 5-\mathrm{H} 5$

$\mathrm{C} 3-\mathrm{C} 6-\mathrm{H} 6 \mathrm{~b}$

3- 6 - $-\mathrm{H} 6 \mathrm{c}$

H6a- $66-\mathrm{H} 6 \mathrm{c}$

$\mathrm{H} 6 \mathrm{~b}-\mathrm{C} 6-\mathrm{H} 6 \mathrm{c}$

C7-O1-H1ol

C13-O7-H1o1
$1.5049(16)$

0.95

$1.3643(16)$

0.95

0.98

0.98

0.98

1.6003

1.6003

1.6003

1.2964 (14)

123.29 (9)

120.41 (9)

$116.29(10)$

120.74 (11)

$120.05(10)$

$119.20(10)$

$118.98(10)$

117.27 (9)

123.75 (11)

119.38

121.25 (11)

119.38

$116.54(10)$

122.15 (11)

$121.31(10)$

118.95

$122.10(10)$

118.95

119.51 (11)

120.24

120.24

109.47

109.47

109.47

109.47

109.47

109.47

$98.33(6)$

$101.65(8)$

$104.71(9)$

99.43 (7)
$\mathrm{C} 7-\mathrm{C} 8$

$\mathrm{C} 7-\mathrm{C} 12$

$\mathrm{C} 8$ - 99

C9- $\mathrm{H} 9$

C9- $\mathrm{C} 10$

C10-C11

C11-H11

C11-C12

$\mathrm{C} 12-\mathrm{C} 13$

$\mathrm{H} 2 \mathrm{a}-\mathrm{H} 2 \mathrm{~b}$

$\mathrm{O} 2-\mathrm{N} 3-\mathrm{O} 3$

$\mathrm{O} 2-\mathrm{N} 3-\mathrm{C} 8$

$\mathrm{O} 3-\mathrm{N} 3-\mathrm{C} 8$

$\mathrm{O} 4-\mathrm{N} 4-\mathrm{O} 5$

$\mathrm{O} 4-\mathrm{N} 4-\mathrm{C} 10$

$\mathrm{O} 5-\mathrm{N} 4-\mathrm{C} 10$

$\mathrm{O} 1-\mathrm{C} 7-\mathrm{C} 8$

$\mathrm{O} 1-\mathrm{C} 7-\mathrm{C} 12$

$\mathrm{C} 8-\mathrm{C} 7-\mathrm{C} 12$

N3- 8 - 77

N3- 8 - $-\mathrm{C} 9$

$\mathrm{C} 7-\mathrm{C} 8-\mathrm{C} 9$

$\mathrm{C} 8-\mathrm{C} 9-\mathrm{H} 9$

$\mathrm{C} 8-\mathrm{C} 9-\mathrm{C} 10$

$\mathrm{H} 9-\mathrm{C} 9-\mathrm{C} 10$

N4- $\mathrm{C} 10-\mathrm{C} 9$

N4- $\mathrm{C} 10-\mathrm{C} 11$

C9- $\mathrm{C} 10-\mathrm{C} 11$

$\mathrm{C} 10-\mathrm{C} 11-\mathrm{H} 11$

$\mathrm{C} 10-\mathrm{C} 11-\mathrm{C} 12$

$\mathrm{H} 11-\mathrm{C} 11-\mathrm{C} 12$

$\mathrm{C} 7-\mathrm{C} 12-\mathrm{C} 11$

$\mathrm{C} 7-\mathrm{C} 12-\mathrm{C} 13$

$\mathrm{C} 11-\mathrm{C} 12-\mathrm{C} 13$

$\mathrm{O} 6-\mathrm{C} 13-\mathrm{O} 7$

$\mathrm{O} 6-\mathrm{C} 13-\mathrm{C} 12$

$\mathrm{O} 7-\mathrm{C} 13-\mathrm{C} 12$

$\mathrm{O} 1-\mathrm{H} 1 \mathrm{o} 7-\mathrm{O} 7$

O7-H1o7-H1ol

$\mathrm{O} 1-\mathrm{H} 1 \mathrm{o} 1-\mathrm{O} 7$

O1-H1o1-H1o7
$1.4197(15)$

$1.4357(14)$

$1.3874(16)$

0.95

$1.3750(15)$

$1.3934(16)$

0.95

1.3787 (16)

$1.4939(15)$

$1.4990(2)$

$122.52(9)$

$119.61(9)$

$117.87(10)$

$123.30(10)$

$118.02(9)$

$118.69(10)$

$124.03(9)$

$119.84(10)$

$116.12(10)$

$121.67(10)$

$116.48(9)$

$121.84(9)$

120.37

$119.26(10)$

120.37

$118.68(10)$

$119.25(9)$

$122.05(10)$

120.64

$118.72(9)$

120.64

$121.98(10)$

$119.00(10)$

$119.02(9)$

$123.12(10)$

$120.31(10)$

$116.57(9)$

$161.55(6)$

$166.67(6)$

$163.52(6)$

$171.56(5)$

Hydrogen-bond geometry $\left(A,{ }^{\circ}\right)$

\begin{tabular}{lllll}
\hline$D-\mathrm{H} \cdots A$ & $D-\mathrm{H}$ & $\mathrm{H} \cdots A$ & $D \cdots A$ & $D-\mathrm{H} \cdots A$ \\
\hline $\mathrm{C} 2-\mathrm{H} 2 \cdots \mathrm{O} 4^{\mathrm{i}}$ & 0.95 & 2.47 & $3.4107(16)$ & 169
\end{tabular}




$\begin{array}{lllll}\mathrm{C} 4-\mathrm{H} 4 \cdots \mathrm{O} 3^{i i} & 0.95 & 2.38 & 3.2397(15) & 151 \\ \mathrm{C} 5-\mathrm{H} 5 \cdots \mathrm{O} 2^{\mathrm{iii}} & 0.95 & 2.43 & 3.2361(16) & 143 \\ \mathrm{O} 7-\mathrm{H} 107 \cdots \mathrm{O} 1 & 0.9185(8) & 1.5313(8) & 2.4202(12) & 161.55(6) \\ \mathrm{O} 1-\mathrm{H} 101 \cdots \mathrm{O} 7 & 0.9310(8) & 1.5130(8) & 2.4202(12) & 163.52(6) \\ \mathrm{N} 2-\mathrm{H} 2 a \cdots \mathrm{O} 7^{\mathrm{iv}} & 0.8921(9) & 2.0783(9) & 2.9655(14) & 172.84(6) \\ \mathrm{N} 2-\mathrm{H} 2 b \cdots \mathrm{O} 1^{i i i} & 0.8456(10) & 2.1644(9) & 2.8526(14) & 138.40(6) \\ \mathrm{N} 2-\mathrm{H} 2 b \cdots \mathrm{O} 2^{i i i} & 0.8456(10) & 2.4133(10) & 3.1741(14) & 150.02(6) \\ \mathrm{N} 1-\mathrm{H} 1 \cdots 6^{i \mathrm{ii}} & 0.8977(9) & 1.7828(9) & 2.6781(13) & 174.83(6)\end{array}$

Symmetry codes: (i) $x-1, y-1, z$; (ii) $-x+1,-y+1,-z+1$; (iii) $x+1, y, z$; (iv) $-x+1,-y+1,-z$.

3,5-Diamino-6-(2,3-dichlorophenyl)-1,2,4-triazin-2-ium 3,5-dinitro-2-hydroxybenzoate N,N-dimethylformamide monosolvate (QIQJAD)

Crystal data

\section{$\mathrm{C}_{9} \mathrm{H}_{8} \mathrm{Cl}_{2} \mathrm{~N}_{5}{ }^{+} \cdot \mathrm{C}_{7} \mathrm{H}_{3} \mathrm{~N}_{2} \mathrm{O}_{7} \cdot \mathrm{C}_{3} \mathrm{H}_{7} \mathrm{NO}$}

$M_{r}=557.31$

Triclinic, $P \overline{1}$

Hall symbol: -P 1

$a=10.0227(5) \AA$

$b=10.5507(5) \AA$

$c=12.5359(6) \AA$

$\alpha=81.858(1)^{\circ}$

$\beta=71.888(1)^{\circ}$

$\gamma=70.009(1)^{\circ}$

$V=1183.1(1) \AA^{3}$

\section{Data collection}

Bruker SMART APEX CCD area-detector diffractometer

Radiation source: fine-focus sealed tube Graphite monochromator

$\omega$ scans

Absorption correction: multi-scan

(SADABS; Bruker, 2001)

$T_{\min }=0.93, T_{\max }=0.97$

\section{Refinement}

Refinement on $F^{2}$

$R[F>3 \sigma(F)]=0.056$

$w R(F)=0.147$

$S=3.41$

5507 reflections

340 parameters

0 restraints

48 constraints

Primary atom site location: structure-invariant direct methods
$Z=2$

$F(000)=572$

$D_{\mathrm{x}}=1.564 \mathrm{Mg} \mathrm{m}^{-3}$

Mo $K \alpha$ radiation, $\lambda=0.71073 \AA$

Cell parameters from 6413 reflections

$\theta=2.3-28.2^{\circ}$

$\mu=0.34 \mathrm{~mm}^{-1}$

$T=294 \mathrm{~K}$

Plate, colourless

$0.16 \times 0.14 \times 0.08 \mathrm{~mm}$

13936 measured reflections

5507 independent reflections

4441 reflections with $I>3 \sigma(I)$

$R_{\text {int }}=0.019$

$\theta_{\text {max }}=28.0^{\circ}, \theta_{\min }=1.7^{\circ}$

$h=-12 \rightarrow 13$

$k=-13 \rightarrow 13$

$l=-16 \rightarrow 16$

Secondary atom site location: difference Fourier map

Hydrogen site location: difference Fourier map

$\mathrm{H}$ atoms treated by a mixture of independent and constrained refinement

Weighting scheme based on measured s.u.'s $w=$ $1 /\left(\sigma^{2}(I)+0.0004 I^{2}\right)$

$(\Delta / \sigma)_{\max }=0.020$

$\Delta \rho_{\max }=0.80 \mathrm{e} \AA^{-3}$

$\Delta \rho_{\min }=-0.36$ e $\AA^{-3}$ 


\section{Special details}

Geometry. All e.s.d.'s (except the e.s.d. in the dihedral angle between two 1.s. planes) are estimated using the full covariance matrix. The cell e.s.d.'s are taken into account individually in the estimation of e.s.d.'s in distances, angles and torsion angles; correlations between e.s.d.'s in cell parameters are only used when they are defined by crystal symmetry. An approximate (isotropic) treatment of cell e.s.d.'s is used for estimating e.s.d.'s involving l.s. planes.

Refinement. Number of fixed parameters: 18

Fractional atomic coordinates and isotropic or equivalent isotropic displacement parameters $\left(\AA^{2}\right)$

\begin{tabular}{|c|c|c|c|c|}
\hline & $x$ & $y$ & $z$ & $U_{\text {iso }} * / U_{\text {eq }}$ \\
\hline $\mathrm{C} 1$ & $0.32107(18)$ & 1.06764 (19) & $0.15844(16)$ & $0.0464(7)$ \\
\hline $\mathrm{C} 2$ & $0.2718(2)$ & $1.0232(2)$ & $0.08378(15)$ & $0.0485(7)$ \\
\hline $\mathrm{C} 3$ & $0.2713(2)$ & 1.0903 (2) & $-0.02046(16)$ & $0.0529(8)$ \\
\hline $\mathrm{C} 4$ & $0.3231(2)$ & $1.2001(2)$ & $-0.04951(19)$ & $0.0621(9)$ \\
\hline $\mathrm{H} 4$ & 0.323127 & 1.245173 & -0.11886 & $0.0745^{*}$ \\
\hline $\mathrm{C} 5$ & $0.3741(2)$ & $1.2420(2)$ & 0.02358 (19) & $0.0632(10)$ \\
\hline H5 & 0.410127 & 1.314857 & 0.001937 & $0.0758 *$ \\
\hline C6 & $0.3745(2)$ & 1.1807 (2) & $0.12857(17)$ & $0.0537(8)$ \\
\hline H6 & 0.40816 & 1.211924 & 0.177727 & $0.0645^{*}$ \\
\hline $\mathrm{C} 7$ & $0.32753(18)$ & 0.99894 (19) & $0.26974(15)$ & $0.0437(7)$ \\
\hline $\mathrm{C} 8$ & $0.35068(19)$ & $0.8912(2)$ & $0.47363(15)$ & $0.0449(7)$ \\
\hline C9 & $0.19780(18)$ & $0.99408(18)$ & $0.36291(15)$ & $0.0418(7)$ \\
\hline N1 & $0.45735(16)$ & $0.95248(17)$ & $0.28531(13)$ & $0.0488(6)$ \\
\hline $\mathrm{N} 2$ & $0.46755(16)$ & $0.89842(17)$ & $0.38722(13)$ & $0.0484(6)$ \\
\hline N3 & $0.37354(18)$ & $0.8352(2)$ & $0.56859(14)$ & $0.0613(8)$ \\
\hline N4 & $0.21299(15)$ & $0.93978(16)$ & $0.46191(12)$ & $0.0454(6)$ \\
\hline N5 & $0.06397(16)$ & $1.04599(17)$ & $0.35102(13)$ & $0.0504(7)$ \\
\hline $\mathrm{Cl1}$ & $0.21444(7)$ & $0.88366(6)$ & $0.11855(5)$ & $0.0685(3)$ \\
\hline $\mathrm{Cl} 2$ & $0.20368(7)$ & $1.04039(8)$ & $-0.11161(5)$ & $0.0772(3)$ \\
\hline $\mathrm{C} 10$ & $0.7671(2)$ & $0.7509(2)$ & $0.50260(17)$ & $0.0495(8)$ \\
\hline C11 & $0.92521(19)$ & $0.68875(18)$ & $0.50532(16)$ & $0.0456(8)$ \\
\hline $\mathrm{C} 12$ & $1.0399(2)$ & $0.68056(19)$ & $0.40501(17)$ & $0.0482(8)$ \\
\hline $\mathrm{C} 13$ & $1.1855(2)$ & $0.6217(2)$ & $0.4120(2)$ & $0.0565(9)$ \\
\hline $\mathrm{C} 14$ & $1.2169(3)$ & $0.5728(2)$ & $0.5120(2)$ & $0.0650(11)$ \\
\hline H14 & 1.31429 & 0.535992 & 0.515021 & $0.078^{*}$ \\
\hline $\mathrm{C} 15$ & $1.1014(3)$ & $0.5795(2)$ & $0.6070(2)$ & $0.0608(10)$ \\
\hline $\mathrm{C} 16$ & $0.9560(2)$ & $0.63637(19)$ & $0.60559(18)$ & $0.0537(9)$ \\
\hline H16 & 0.879592 & 0.639442 & 0.671313 & $0.0645^{*}$ \\
\hline N6 & $1.3082(2)$ & $0.6096(2)$ & $0.3083(2)$ & $0.0763(10)$ \\
\hline N7 & $1.1324(3)$ & $0.5213(2)$ & $0.7146(3)$ & $0.0870(14)$ \\
\hline $\mathrm{O} 1$ & $0.74835(14)$ & $0.79734(16)$ & $0.40757(12)$ & $0.0638(7)$ \\
\hline $\mathrm{O} 2$ & $0.66684(16)$ & $0.75284(17)$ & $0.58991(13)$ & $0.0695(7)$ \\
\hline $\mathrm{O} 3$ & $1.01381(16)$ & $0.72881(16)$ & $0.30779(13)$ & $0.0651(7)$ \\
\hline $\mathrm{O} 4$ & $1.3027(2)$ & 0.5624 & $0.22939(18)$ & $0.0962(10)$ \\
\hline $\mathrm{O} 5$ & $1.4096(2)$ & 0.6460 & $0.3106(2)$ & $0.1311(14)$ \\
\hline O6 & $1.2621(3)$ & 0.4654 & $0.7114(2)$ & $0.1272(14)$ \\
\hline $\mathrm{O} 7$ & $1.0297(3)$ & $0.5356(3)$ & $0.7980(2)$ & $0.1153(15)$ \\
\hline $\mathrm{C} 17$ & $0.8968(2)$ & $0.2419(2)$ & $0.13898(19)$ & $0.0638(10)$ \\
\hline
\end{tabular}




$\begin{array}{lllll}\text { H17 } & 0.997049 & 0.225753 & 0.10284 & 0.0765^{*} \\ \text { C18 } & 0.6486(3) & 0.3564(4) & 0.1366(3) & 0.1002(16) \\ \text { H18a } & 0.600566 & 0.362237 & 0.079827 & 0.1503^{*} \\ \text { H18b } & 0.612084 & 0.441665 & 0.171497 & 0.1503^{*} \\ \text { H18c } & 0.628154 & 0.287766 & 0.192178 & 0.1503^{*} \\ \text { C19 } & 0.8613(4) & 0.3905(3) & -0.0204(2) & 0.0959(16) \\ \text { H19a } & 0.812684 & 0.38463 & -0.07367 & 0.1439^{*} \\ \text { H19b } & 0.842404 & 0.483699 & -0.008394 & 0.1439^{*} \\ \text { H19c } & 0.965894 & 0.347377 & -0.048929 & 0.1439^{*} \\ \text { N8 } & 0.8043(2) & 0.32305(17) & 0.08649(14) & 0.0588(8) \\ \text { O8 } & 0.86513(17) & 0.18476(18) & 0.23111(12) & 0.0693(7) \\ \text { H3n } & 0.459992 & 0.808642 & 0.579383 & 0.067(7)^{*} \\ \text { H4n } & 0.300684 & 0.831649 & 0.62665 & 0.058(6)^{*} \\ \text { H2n } & 0.560448 & 0.867423 & 0.392878 & 0.069(7)^{*} \\ \text { H5n } & -0.01125 & 1.040769 & 0.406749 & 0.056(6)^{*} \\ \text { H6n } & 0.043449 & 1.086703 & 0.2906 & 0.052(6)^{*} \\ \text { H3o } & 0.911378 & 0.756483 & 0.330791 & 0.131(12)^{*} \\ \end{array}$

Atomic displacement parameters $\left(A^{2}\right)$

\begin{tabular}{lllllll}
\hline & $U^{11}$ & $U^{22}$ & $U^{33}$ & $U^{12}$ & $U^{13}$ & $U^{23}$ \\
\hline C1 & $0.0300(8)$ & $0.0541(11)$ & $0.0483(10)$ & $-0.0081(8)$ & $-0.0060(7)$ & $-0.0055(8)$ \\
C2 & $0.0354(9)$ & $0.0578(11)$ & $0.0464(10)$ & $-0.0118(8)$ & $-0.0044(8)$ & $-0.0068(8)$ \\
C3 & $0.0364(9)$ & $0.0682(13)$ & $0.0443(10)$ & $-0.0090(9)$ & $-0.0049(8)$ & $-0.0047(9)$ \\
C4 & $0.0457(11)$ & $0.0737(15)$ & $0.0546(12)$ & $-0.0053(10)$ & $-0.0119(9)$ & $-0.0038(11)$ \\
C5 & $0.0580(13)$ & $0.0599(13)$ & $0.0701(14)$ & $-0.0224(11)$ & $-0.0153(11)$ & $0.0054(11)$ \\
C6 & $0.0442(10)$ & $0.0589(12)$ & $0.0502(11)$ & $-0.0107(9)$ & $-0.0112(9)$ & $0.0032(9)$ \\
C7 & $0.0323(9)$ & $0.0527(11)$ & $0.0433(10)$ & $-0.0115(8)$ & $-0.0076(7)$ & $-0.0053(8)$ \\
C8 & $0.0316(8)$ & $0.0564(11)$ & $0.0446(10)$ & $-0.0113(8)$ & $-0.0096(7)$ & $-0.0050(8)$ \\
C9 & $0.0305(8)$ & $0.0502(10)$ & $0.0430(9)$ & $-0.0112(7)$ & $-0.0074(7)$ & $-0.0072(8)$ \\
N1 & $0.0326(8)$ & $0.0622(10)$ & $0.0469(9)$ & $-0.0126(7)$ & $-0.0079(6)$ & $-0.0007(7)$ \\
N2 & $0.0282(7)$ & $0.0679(10)$ & $0.0450(9)$ & $-0.0113(7)$ & $-0.0098(6)$ & $-0.0005(7)$ \\
N3 & $0.0343(8)$ & $0.0949(14)$ & $0.0468(9)$ & $-0.0152(9)$ & $-0.0110(7)$ & $0.0078(9)$ \\
N4 & $0.0293(7)$ & $0.0618(10)$ & $0.0417(8)$ & $-0.0119(7)$ & $-0.0077(6)$ & $-0.0027(7)$ \\
N5 & $0.0307(7)$ & $0.0730(11)$ & $0.0421(9)$ & $-0.0106(7)$ & $-0.0102(7)$ & $-0.0001(8)$ \\
C11 & $0.0784(4)$ & $0.0786(4)$ & $0.0600(3)$ & $-0.0419(3)$ & $-0.0150(3)$ & $-0.0059(3)$ \\
C12 & $0.0721(4)$ & $0.1162(5)$ & $0.0522(3)$ & $-0.0373(4)$ & $-0.0190(3)$ & $-0.0090(3)$ \\
C10 & $0.0386(10)$ & $0.0504(11)$ & $0.0597(12)$ & $-0.0073(8)$ & $-0.0180(9)$ & $-0.0107(9)$ \\
C11 & $0.0404(10)$ & $0.0417(10)$ & $0.0597(12)$ & $-0.0103(8)$ & $-0.0219(9)$ & $-0.0067(8)$ \\
C12 & $0.0395(10)$ & $0.0464(10)$ & $0.0641(12)$ & $-0.0136(8)$ & $-0.0218(9)$ & $-0.0030(9)$ \\
C13 & $0.0384(10)$ & $0.0512(11)$ & $0.0826(15)$ & $-0.0101(8)$ & $-0.0220(10)$ & $-0.0101(10)$ \\
C14 & $0.0504(12)$ & $0.0533(12)$ & $0.1065(19)$ & $-0.0093(10)$ & $-0.0465(13)$ & $-0.0135(12)$ \\
C15 & $0.0692(15)$ & $0.0506(12)$ & $0.0797(15)$ & $-0.0144(10)$ & $-0.0492(13)$ & $-0.0036(10)$ \\
C16 & $0.0578(12)$ & $0.0501(11)$ & $0.0625(13)$ & $-0.0162(9)$ & $-0.0287(10)$ & $-0.0069(9)$ \\
N6 & $0.0372(10)$ & $0.0779(14)$ & $0.1062(18)$ & $-0.0082(9)$ & $-0.0156(10)$ & $-0.0157(12)$ \\
N7 & $0.113(2)$ & $0.0759(15)$ & $0.1066(19)$ & $-0.0325(14)$ & $-0.0800(17)$ & $0.0073(14)$ \\
O1 & $0.0374(7)$ & $0.0841(11)$ & $0.0645(9)$ & $-0.0093(7)$ & $-0.0222(7)$ & $0.0067(8)$ \\
O2 & $0.0428(8)$ & $0.0946(12)$ & $0.0601(9)$ & $-0.0074(8)$ & $-0.0121(7)$ & $-0.0108(8)$ \\
& & & & & &
\end{tabular}




\begin{tabular}{lllllll}
\hline O3 & $0.0427(8)$ & $0.0802(10)$ & $0.0653(9)$ & $-0.0151(7)$ & $-0.0148(7)$ & $0.0074(8)$ \\
O4 & $0.0575(10)$ & $0.1277(17)$ & $0.0882(13)$ & $-0.0110(11)$ & $-0.0135(9)$ & $-0.0195(12)$ \\
O5 & $0.0520(11)$ & $0.164(2)$ & $0.181(2)$ & $-0.0480(13)$ & $0.0026(13)$ & $-0.0623(18)$ \\
O6 & $0.1283(19)$ & $0.1273(18)$ & $0.142(2)$ & $0.0037(15)$ & $-0.1080(17)$ & $-0.0100(15)$ \\
O7 & $0.142(2)$ & $0.159(2)$ & $0.0879(15)$ & $-0.0849(19)$ & $-0.0675(16)$ & $0.0370(15)$ \\
C17 & $0.0454(11)$ & $0.0753(15)$ & $0.0575(13)$ & $-0.0065(10)$ & $-0.0078(10)$ & $-0.0085(11)$ \\
C18 & $0.0568(15)$ & $0.129(3)$ & $0.120(2)$ & $-0.0180(16)$ & $-0.0417(16)$ & $-0.009(2)$ \\
C19 & $0.123(3)$ & $0.0831(19)$ & $0.0697(17)$ & $-0.0251(18)$ & $-0.0261(16)$ & $0.0120(14)$ \\
N8 & $0.0593(11)$ & $0.0601(11)$ & $0.0548(10)$ & $-0.0096(9)$ & $-0.0229(9)$ & $-0.0033(8)$ \\
O8 & $0.0558(9)$ & $0.0937(12)$ & $0.0520(9)$ & $-0.0195(8)$ & $-0.0165(7)$ & $0.0097(8)$ \\
\hline
\end{tabular}

Geometric parameters $\left(A,{ }^{\circ}\right)$

\begin{tabular}{|c|c|c|c|}
\hline $\mathrm{C} 1-\mathrm{C} 2$ & $1.382(3)$ & $\mathrm{C} 13-\mathrm{C} 14$ & $1.374(4)$ \\
\hline $\mathrm{C} 1-\mathrm{C} 6$ & $1.427(3)$ & $\mathrm{C} 13-\mathrm{N} 6$ & $1.471(3)$ \\
\hline $\mathrm{C} 1-\mathrm{C} 7$ & $1.488(3)$ & $\mathrm{C} 14-\mathrm{H} 14$ & 0.93 \\
\hline $\mathrm{C} 2-\mathrm{C} 3$ & $1.396(3)$ & $\mathrm{C} 14-\mathrm{C} 15$ & $1.369(3)$ \\
\hline $\mathrm{C} 3-\mathrm{C} 4$ & $1.385(4)$ & $\mathrm{C} 15-\mathrm{C} 16$ & $1.378(3)$ \\
\hline $\mathrm{C} 4-\mathrm{H} 4$ & 0.93 & $\mathrm{C} 15-\mathrm{N} 7$ & $1.479(4)$ \\
\hline $\mathrm{C} 4-\mathrm{C} 5$ & $1.362(4)$ & $\mathrm{C} 16-\mathrm{H} 16$ & 0.93 \\
\hline $\mathrm{C} 5-\mathrm{H} 5$ & 0.93 & $\mathrm{~N} 6-\mathrm{O} 4$ & $1.192(4)$ \\
\hline $\mathrm{C} 5-\mathrm{C} 6$ & $1.382(3)$ & $\mathrm{N} 6-\mathrm{O} 5$ & $1.213(4)$ \\
\hline $\mathrm{C} 6-\mathrm{H} 6$ & 0.93 & N7-O6 & $1.221(4)$ \\
\hline $\mathrm{C} 7-\mathrm{C} 9$ & $1.464(2)$ & $\mathrm{N} 7-\mathrm{O} 7$ & $1.202(4)$ \\
\hline $\mathrm{C} 7-\mathrm{N} 1$ & $1.291(2)$ & $\mathrm{O} 3-\mathrm{H} 3 \mathrm{o}$ & $0.9258(14)$ \\
\hline $\mathrm{C} 8-\mathrm{N} 2$ & $1.342(2)$ & $\mathrm{C} 17-\mathrm{H} 17$ & 0.93 \\
\hline $\mathrm{C} 8-\mathrm{N} 3$ & $1.303(3)$ & $\mathrm{C} 17-\mathrm{N} 8$ & $1.305(3)$ \\
\hline $\mathrm{C} 8-\mathrm{N} 4$ & $1.345(2)$ & $\mathrm{C} 17-\mathrm{O} 8$ & $1.226(3)$ \\
\hline $\mathrm{C} 9-\mathrm{N} 4$ & $1.322(2)$ & $\mathrm{C} 18-\mathrm{H} 18 \mathrm{a}$ & 0.96 \\
\hline $\mathrm{C} 9-\mathrm{N} 5$ & $1.312(2)$ & $\mathrm{C} 18-\mathrm{H} 18 \mathrm{~b}$ & 0.96 \\
\hline $\mathrm{N} 1-\mathrm{N} 2$ & $1.343(2)$ & $\mathrm{C} 18-\mathrm{H} 18 \mathrm{c}$ & 0.96 \\
\hline $\mathrm{N} 2-\mathrm{H} 2 \mathrm{n}$ & $0.8973(16)$ & $\mathrm{C} 18-\mathrm{N} 8$ & $1.425(3)$ \\
\hline N3-H3n & $0.8624(18)$ & $\mathrm{H} 18 \mathrm{a}-\mathrm{H} 18 \mathrm{~b}$ & 1.5677 \\
\hline $\mathrm{N} 3-\mathrm{H} 4 \mathrm{n}$ & $0.8630(15)$ & $\mathrm{H} 18 \mathrm{a}-\mathrm{H} 18 \mathrm{c}$ & 1.5677 \\
\hline N5-H5n & $0.8658(14)$ & $\mathrm{H} 18 \mathrm{~b}-\mathrm{H} 18 \mathrm{c}$ & 1.5677 \\
\hline N5-H6n & $0.8630(16)$ & $\mathrm{C} 19-\mathrm{H} 19 \mathrm{a}$ & 0.96 \\
\hline $\mathrm{C} 10-\mathrm{C} 11$ & $1.503(3)$ & $\mathrm{C} 19-\mathrm{H} 19 \mathrm{~b}$ & 0.96 \\
\hline $\mathrm{C} 10-\mathrm{O} 1$ & $1.267(3)$ & $\mathrm{C} 19-\mathrm{H} 19 \mathrm{c}$ & 0.96 \\
\hline $\mathrm{C} 10-\mathrm{O} 2$ & $1.231(2)$ & $\mathrm{C} 19-\mathrm{N} 8$ & $1.470(3)$ \\
\hline $\mathrm{C} 11-\mathrm{C} 12$ & $1.405(2)$ & $\mathrm{H} 19 \mathrm{a}-\mathrm{H} 19 \mathrm{~b}$ & 1.5677 \\
\hline $\mathrm{C} 11-\mathrm{C} 16$ & $1.382(3)$ & $\mathrm{H} 19 \mathrm{a}-\mathrm{H} 19 \mathrm{c}$ & 1.5677 \\
\hline $\mathrm{C} 12-\mathrm{C} 13$ & $1.402(3)$ & $\mathrm{H} 19 \mathrm{~b}-\mathrm{H} 19 \mathrm{c}$ & 1.5677 \\
\hline $\mathrm{C} 12-\mathrm{O} 3$ & $1.321(3)$ & & \\
\hline $\mathrm{C} 2-\mathrm{C} 1-\mathrm{C} 6$ & $120.20(18)$ & $\mathrm{C} 11-\mathrm{C} 12-\mathrm{O} 3$ & $122.09(17)$ \\
\hline $\mathrm{C} 2-\mathrm{C} 1-\mathrm{C} 7$ & $122.86(19)$ & $\mathrm{C} 13-\mathrm{C} 12-\mathrm{O} 3$ & $120.57(17)$ \\
\hline $\mathrm{C} 6-\mathrm{C} 1-\mathrm{C} 7$ & $116.9(2)$ & $\mathrm{C} 12-\mathrm{C} 13-\mathrm{C} 14$ & $122.17(18)$ \\
\hline $\mathrm{C} 1-\mathrm{C} 2-\mathrm{C} 3$ & $120.2(2)$ & $\mathrm{C} 12-\mathrm{C} 13-\mathrm{N} 6$ & $118.7(2)$ \\
\hline
\end{tabular}




\begin{tabular}{|c|c|c|c|}
\hline $\mathrm{C} 2-\mathrm{C} 3-\mathrm{C} 4$ & $119.6(2)$ & $\mathrm{C} 14-\mathrm{C} 13-\mathrm{N} 6$ & $119.10(19)$ \\
\hline $\mathrm{C} 3-\mathrm{C} 4-\mathrm{H} 4$ & 120.02 & $\mathrm{C} 13-\mathrm{C} 14-\mathrm{H} 14$ & 120.79 \\
\hline $\mathrm{C} 3-\mathrm{C} 4-\mathrm{C} 5$ & $120.0(2)$ & $\mathrm{C} 13-\mathrm{C} 14-\mathrm{C} 15$ & $118.4(2)$ \\
\hline $\mathrm{H} 4-\mathrm{C} 4-\mathrm{C} 5$ & 120.02 & $\mathrm{H} 14-\mathrm{C} 14-\mathrm{C} 15$ & 120.79 \\
\hline $\mathrm{C} 4-\mathrm{C} 5-\mathrm{H} 5$ & 118.63 & $\mathrm{C} 14-\mathrm{C} 15-\mathrm{C} 16$ & $122.1(2)$ \\
\hline $\mathrm{C} 4-\mathrm{C} 5-\mathrm{C} 6$ & $122.7(2)$ & $\mathrm{C} 14-\mathrm{C} 15-\mathrm{N} 7$ & $119.4(2)$ \\
\hline $\mathrm{H} 5-\mathrm{C} 5-\mathrm{C} 6$ & 118.63 & $\mathrm{C} 16-\mathrm{C} 15-\mathrm{N} 7$ & $118.5(2)$ \\
\hline $\mathrm{C} 1-\mathrm{C} 6-\mathrm{C} 5$ & $117.3(2)$ & $\mathrm{C} 11-\mathrm{C} 16-\mathrm{C} 15$ & $119.19(18)$ \\
\hline $\mathrm{C} 1-\mathrm{C} 6-\mathrm{H} 6$ & 121.37 & $\mathrm{C} 11-\mathrm{C} 16-\mathrm{H} 16$ & 120.41 \\
\hline $\mathrm{C} 5-\mathrm{C} 6-\mathrm{H} 6$ & 121.36 & $\mathrm{C} 15-\mathrm{C} 16-\mathrm{H} 16$ & 120.41 \\
\hline $\mathrm{C} 1-\mathrm{C} 7-\mathrm{C} 9$ & $124.50(16)$ & $\mathrm{C} 13-\mathrm{N} 6-\mathrm{O} 4$ & $118.2(2)$ \\
\hline $\mathrm{C} 1-\mathrm{C} 7-\mathrm{N} 1$ & $115.60(15)$ & $\mathrm{C} 13-\mathrm{N} 6-\mathrm{O} 5$ & 117.0 \\
\hline $\mathrm{C} 9-\mathrm{C} 7-\mathrm{N} 1$ & $119.58(16)$ & $\mathrm{O} 4-\mathrm{N} 6-\mathrm{O} 5$ & $124.8(2)$ \\
\hline $\mathrm{N} 2-\mathrm{C} 8-\mathrm{N} 3$ & $118.61(17)$ & $\mathrm{C} 15-\mathrm{N} 7-\mathrm{O} 6$ & $116.6(2)$ \\
\hline $\mathrm{N} 2-\mathrm{C} 8-\mathrm{N} 4$ & $120.57(17)$ & $\mathrm{C} 15-\mathrm{N} 7-\mathrm{O} 7$ & $118.1(3)$ \\
\hline $\mathrm{N} 3-\mathrm{C} 8-\mathrm{N} 4$ & $120.81(16)$ & $\mathrm{O} 6-\mathrm{N} 7-\mathrm{O} 7$ & $125.3(3)$ \\
\hline $\mathrm{C} 7-\mathrm{C} 9-\mathrm{N} 4$ & $120.53(16)$ & $\mathrm{C} 10-\mathrm{O} 1-\mathrm{H} 3 \mathrm{o}$ & $101.27(12)$ \\
\hline $\mathrm{C} 7-\mathrm{C} 9-\mathrm{N} 5$ & $120.93(16)$ & $\mathrm{C} 12-\mathrm{O} 3-\mathrm{H} 3 \mathrm{o}$ & $99.28(14)$ \\
\hline $\mathrm{N} 4-\mathrm{C} 9-\mathrm{N} 5$ & $118.52(15)$ & $\mathrm{H} 17-\mathrm{C} 17-\mathrm{N} 8$ & 116.69 \\
\hline $\mathrm{C} 7-\mathrm{N} 1-\mathrm{N} 2$ & $117.85(14)$ & $\mathrm{H} 17-\mathrm{C} 17-\mathrm{O} 8$ & 116.69 \\
\hline $\mathrm{C} 8-\mathrm{N} 2-\mathrm{N} 1$ & $123.77(16)$ & $\mathrm{N} 8-\mathrm{C} 17-\mathrm{O} 8$ & $126.6(2)$ \\
\hline $\mathrm{C} 8-\mathrm{N} 2-\mathrm{H} 2 \mathrm{n}$ & $122.42(17)$ & $\mathrm{H} 18 \mathrm{a}-\mathrm{C} 18-\mathrm{H} 18 \mathrm{~b}$ & 109.47 \\
\hline $\mathrm{N} 1-\mathrm{N} 2-\mathrm{H} 2 \mathrm{n}$ & $113.82(14)$ & $\mathrm{H} 18 \mathrm{a}-\mathrm{C} 18-\mathrm{H} 18 \mathrm{c}$ & 109.47 \\
\hline $\mathrm{C} 8-\mathrm{N} 3-\mathrm{H} 3 \mathrm{n}$ & $122.28(17)$ & $\mathrm{H} 18 \mathrm{a}-\mathrm{C} 18-\mathrm{N} 8$ & 109.47 \\
\hline $\mathrm{C} 8-\mathrm{N} 3-\mathrm{H} 4 \mathrm{n}$ & $121.07(18)$ & $\mathrm{H} 18 \mathrm{~b}-\mathrm{C} 18-\mathrm{H} 18 \mathrm{c}$ & 109.47 \\
\hline $\mathrm{H} 3 n-\mathrm{N} 3-\mathrm{H} 4 \mathrm{n}$ & $116.2(2)$ & $\mathrm{H} 18 \mathrm{~b}-\mathrm{C} 18-\mathrm{N} 8$ & 109.47 \\
\hline $\mathrm{C} 8-\mathrm{N} 4-\mathrm{C} 9$ & $117.69(14)$ & $\mathrm{H} 18 \mathrm{c}-\mathrm{C} 18-\mathrm{N} 8$ & 109.47 \\
\hline C9-N5-H5n & $119.55(17)$ & $\mathrm{H} 19 \mathrm{a}-\mathrm{C} 19-\mathrm{H} 19 \mathrm{~b}$ & 109.47 \\
\hline $\mathrm{C} 9-\mathrm{N} 5-\mathrm{H} 6 \mathrm{n}$ & $124.81(16)$ & $\mathrm{H} 19 \mathrm{a}-\mathrm{C} 19-\mathrm{H} 19 \mathrm{c}$ & 109.47 \\
\hline $\mathrm{H} 5 \mathrm{n}-\mathrm{N} 5-\mathrm{H} 6 \mathrm{n}$ & $115.64(18)$ & $\mathrm{H} 19 \mathrm{a}-\mathrm{C} 19-\mathrm{N} 8$ & 109.47 \\
\hline $\mathrm{C} 11-\mathrm{C} 10-\mathrm{O} 1$ & $115.76(15)$ & $\mathrm{H} 19 \mathrm{~b}-\mathrm{C} 19-\mathrm{H} 19 \mathrm{c}$ & 109.47 \\
\hline $\mathrm{C} 11-\mathrm{C} 10-\mathrm{O} 2$ & $119.27(19)$ & $\mathrm{H} 19 \mathrm{~b}-\mathrm{C} 19-\mathrm{N} 8$ & 109.47 \\
\hline $\mathrm{O} 1-\mathrm{C} 10-\mathrm{O} 2$ & $124.96(19)$ & $\mathrm{H} 19 \mathrm{c}-\mathrm{C} 19-\mathrm{N} 8$ & 109.47 \\
\hline $\mathrm{C} 10-\mathrm{C} 11-\mathrm{C} 12$ & $119.51(18)$ & $\mathrm{C} 17-\mathrm{N} 8-\mathrm{C} 18$ & $120.7(2)$ \\
\hline $\mathrm{C} 10-\mathrm{C} 11-\mathrm{C} 16$ & $119.71(16)$ & $\mathrm{C} 17-\mathrm{N} 8-\mathrm{C} 19$ & $119.5(2)$ \\
\hline $\mathrm{C} 12-\mathrm{C} 11-\mathrm{C} 16$ & $120.74(18)$ & $\mathrm{C} 18-\mathrm{N} 8-\mathrm{C} 19$ & $119.6(2)$ \\
\hline $\mathrm{C} 11-\mathrm{C} 12-\mathrm{C} 13$ & $117.3(2)$ & $\mathrm{O} 1-\mathrm{H} 3 \mathrm{o}-\mathrm{O} 3$ & $161.33(12)$ \\
\hline
\end{tabular}

Hydrogen-bond geometry $\left(\AA,{ }^{\circ}\right)$

\begin{tabular}{lllll}
\hline$D-\mathrm{H} \cdots A$ & $D-\mathrm{H}$ & $\mathrm{H} \cdots A$ & $D \cdots A$ & $D-\mathrm{H} \cdots A$ \\
\hline $\mathrm{C} 19-\mathrm{H} 19 a \cdots \mathrm{O} 4^{\mathrm{i}}$ & 0.96 & 2.47 & $3.401(4)$ & 163 \\
$\mathrm{~N} 3-\mathrm{H} 3 n \cdots \mathrm{O} 2$ & $0.8624(18)$ & $1.9939(16)$ & $2.854(2)$ & $174.78(11)$ \\
$\mathrm{N} 3-\mathrm{H} 4 n \cdots \mathrm{O} 8^{\mathrm{ii}}$ & $0.8630(15)$ & $2.0586(14)$ & $2.921(2)$ & $176.91(12)$ \\
$\mathrm{N} 2-\mathrm{H} 2 n \cdots \mathrm{O} 1$ & $0.8973(16)$ & $1.8310(15)$ & $2.728(2)$ & $177.45(12)$ \\
$\mathrm{N} 5-\mathrm{H} 5 n \cdots \mathrm{N} 44^{\mathrm{iii}}$ & $0.8658(14)$ & $2.1409(13)$ & $2.9992(19)$ & $171.06(11)$
\end{tabular}




$\begin{array}{lllll}\mathrm{N} 5-\mathrm{H} 6 n^{\cdots} \cdots 8^{\text {iv }} & 0.8630(16) & 2.0412(16) & 2.760(2) & 140.22(10) \\ \mathrm{O} 3-\mathrm{H} 30 \cdots \mathrm{O} 1 & 0.9258(14) & 1.5621(12) & 2.4572(18) & 161.33(12)\end{array}$

Symmetry codes: (i) $-x+2,-y+1,-z$; (ii) $-x+1,-y+1,-z+1$; (iii) $-x,-y+2,-z+1$; (iv) $x-1, y+1, z$.

Bis(1,10-phenanthroline-5,6-dione- $\left.\kappa^{2} N, N^{\prime}\right)$ silver(I) 3,5-dinitrosalicylate (SAFGUD)

\section{Crystal data}

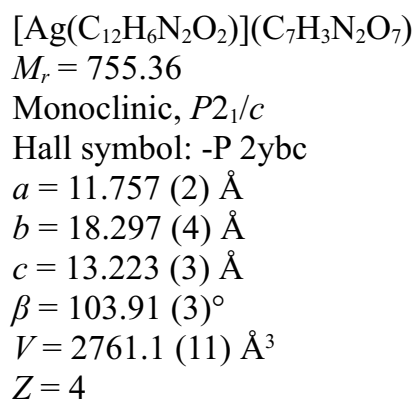

\section{Data collection}

Oxford Diffraction Gemini R Ultra diffractometer

Radiation source: fine-focus sealed tube Graphite monochromator $\omega$ scans

Absorption correction: multi-scan (SADABS; Bruker, 2002)

$T_{\min }=0.780, T_{\max }=0.910$

\section{Refinement}

Refinement on $F^{2}$

Least-squares matrix: full

$R[F>3 \sigma(F)]=0.062$

$w R(F)=0.118$

$S=1.64$

5013 reflections

444 parameters

0 restraints

56 constraints

Primary atom site location: structure-invariant direct methods
$F(000)=1512$
$D_{\mathrm{x}}=1.817 \mathrm{Mg} \mathrm{m}^{-3}$

Mo $K \alpha$ radiation, $\lambda=0.71073 \AA$

Cell parameters from 5197 reflections

$\theta=3.2-25.4^{\circ}$

$\mu=0.81 \mathrm{~mm}^{-1}$

$T=174 \mathrm{~K}$

Prism, yellow

$0.3 \times 0.24 \times 0.2 \mathrm{~mm}$

12726 measured reflections

5013 independent reflections

3100 reflections with $I>3 \sigma(I)$

$R_{\text {int }}=0.052$

$\theta_{\max }=25.4^{\circ}, \theta_{\min }=3.2^{\circ}$

$h=-14 \rightarrow 11$

$k=-17 \rightarrow 22$

$l=-15 \rightarrow 13$

Secondary atom site location: difference Fourier map

Hydrogen site location: inferred from neighbouring sites

$\mathrm{H}$-atom parameters constrained

Weighting scheme based on measured s.u.'s $w=$ $1 /\left(\sigma^{2}(I)+0.0004 I^{2}\right)$

$(\Delta / \sigma)_{\max }=0.016$

$\Delta \rho_{\max }=0.76 \mathrm{e}^{-3}$

$\Delta \rho_{\min }=-0.63$ e $\AA^{-3}$

Extinction correction: B-C type 1 Lorentzian isotropic (Becker \& Coppens, 1974)

Extinction coefficient: 2400 (800)

\section{Special details}

Geometry. All esds (except the esd in the dihedral angle between two 1.s. planes) are estimated using the full covariance matrix. The cell esds are taken into account individually in the estimation of esds in distances, angles and torsion angles; correlations between esds in cell parameters are only used when they are defined by crystal symmetry. An approximate (isotropic) treatment of cell esds is used for estimating esds involving l.s. planes.

Refinement. Refinement of $\mathrm{F}^{2}$ against ALL reflections. The weighted R-factor $\mathrm{wR}$ and goodness of fit $\mathrm{S}$ are based on $\mathrm{F}^{2}$, conventional R-factors $R$ are based on $F$, with $F$ set to zero for negative $F^{2}$. The threshold expression of $F^{2}>2 \operatorname{sigma}\left(\mathrm{F}^{2}\right)$ is used only for calculating R-factors(gt) etc. and is not relevant to the choice of reflections for refinement. R-factors based on $\mathrm{F}^{2}$ are statistically about twice as large as those based on $\mathrm{F}$, and $\mathrm{R}$ - factors based on ALL data will be even larger. Number of fixed parameters 3 
Fractional atomic coordinates and isotropic or equivalent isotropic displacement parameters $\left(\AA^{2}\right)$

\begin{tabular}{|c|c|c|c|c|}
\hline & $x$ & $y$ & $z$ & $U_{\text {iso }} * / U_{\text {eq }}$ \\
\hline Ag1 & 0.21070 & 0.44031 & $0.52479(4)$ & $0.0538(2)$ \\
\hline $\mathrm{C} 1$ & $0.4796(5)$ & $0.5138(3)$ & $0.6221(4)$ & $0.043(2)$ \\
\hline H1 & 0.440696 & 0.558209 & 0.60689 & $0.0511^{*}$ \\
\hline $\mathrm{C} 2$ & $0.5975(6)$ & 0.5153 & $0.6692(4)$ & $0.046(2)$ \\
\hline $\mathrm{H} 2$ & 0.637009 & 0.559483 & 0.684873 & $0.0553 *$ \\
\hline $\mathrm{C} 3$ & $0.6551(5)$ & 0.4500 & $0.6925(4)$ & $0.046(2)$ \\
\hline H3 & 0.734391 & 0.449118 & 0.725729 & $0.0547^{*}$ \\
\hline $\mathrm{C} 4$ & $0.5946(5)$ & 0.3859 & $0.6661(4)$ & $0.035(2)$ \\
\hline $\mathrm{C} 5$ & $0.6523(8)$ & $0.3141(4)$ & $0.6884(5)$ & 0.068 \\
\hline C6 & $0.5912(10)$ & 0.2510 & $0.6718(6)$ & $0.093(5)$ \\
\hline $\mathrm{C} 7$ & $0.4628(6)$ & 0.2534 & 0.6228 & $0.042(2)$ \\
\hline $\mathrm{C} 8$ & $0.3994(7)$ & $0.1881(3)$ & $0.6033(4)$ & $0.052(3)$ \\
\hline H8 & 0.435089 & 0.143184 & 0.6224 & $0.0623^{*}$ \\
\hline C9 & $0.2837(7)$ & 0.1928 & 0.5555 & $0.063(3)$ \\
\hline H9 & 0.238665 & 0.150616 & 0.540161 & $0.0756^{*}$ \\
\hline $\mathrm{C} 10$ & $0.2345(6)$ & 0.2592 & $0.5304(5)$ & $0.052(3)$ \\
\hline H10 & 0.155582 & 0.26107 & 0.496188 & $0.0629 *$ \\
\hline C11 & $0.4065(5)$ & 0.3197 & $0.5966(4)$ & $0.032(2)$ \\
\hline $\mathrm{C} 12$ & $0.4742(5)$ & $0.3885(3)$ & $0.6197(4)$ & $0.033(2)$ \\
\hline $\mathrm{C} 13$ & $0.0851(5)$ & $0.4150(3)$ & $0.2774(5)$ & $0.054(3)$ \\
\hline H13 & 0.109147 & 0.367048 & 0.293174 & $0.0649^{*}$ \\
\hline $\mathrm{C} 14$ & $0.0376(5)$ & $0.4324(4)$ & $0.1743(5)$ & $0.060(3)$ \\
\hline H14 & 0.029151 & 0.397085 & 0.122358 & $0.0717^{*}$ \\
\hline $\mathrm{C} 15$ & $0.0032(5)$ & $0.5033(3)$ & $0.1508(5)$ & $0.051(3)$ \\
\hline H15 & -0.030371 & 0.516779 & 0.082274 & $0.0617^{*}$ \\
\hline $\mathrm{C} 16$ & $0.0190(5)$ & 0.5550 & $0.2306(4)$ & $0.040(2)$ \\
\hline $\mathrm{C} 17$ & $-0.0145(5)$ & $0.6317(3)$ & $0.2063(5)$ & $0.051(3)$ \\
\hline $\mathrm{C} 18$ & $-0.0094(5)$ & $0.6826(3)$ & $0.2940(5)$ & $0.053(3)$ \\
\hline C19 & $0.0474(5)$ & 0.6578 & $0.4023(5)$ & $0.042(2)$ \\
\hline $\mathrm{C} 20$ & 0.0689 & 0.7058 & $0.4858(5)$ & $0.052(3)$ \\
\hline $\mathrm{H} 20$ & 0.048099 & 0.754726 & 0.475014 & $0.063^{*}$ \\
\hline $\mathrm{C} 21$ & $0.1204(6)$ & $0.6817(4)$ & $0.5833(5)$ & $0.058(3)$ \\
\hline $\mathrm{H} 21$ & 0.134626 & 0.713123 & 0.64029 & $0.0692 *$ \\
\hline $\mathrm{C} 22$ & $0.1503(5)$ & $0.6091(4)$ & $0.5941(5)$ & $0.054(3)$ \\
\hline $\mathrm{H} 22$ & 0.186352 & 0.59254 & 0.660553 & $0.0653^{*}$ \\
\hline $\mathrm{C} 23$ & $0.0818(5)$ & $0.5847(3)$ & 0.4195 & $0.036(2)$ \\
\hline $\mathrm{C} 24$ & $0.0637(4)$ & 0.5318 & $0.3326(4)$ & $0.032(2)$ \\
\hline $\mathrm{C} 25$ & $0.6150(6)$ & $0.4142(3)$ & $0.9276(4)$ & $0.041(2)$ \\
\hline $\mathrm{C} 26$ & $0.5079(6)$ & 0.4527 & $0.8869(4)$ & $0.040(2)$ \\
\hline $\mathrm{C} 27$ & $0.4074(5)$ & $0.4101(3)$ & $0.8426(4)$ & $0.037(2)$ \\
\hline $\mathrm{C} 28$ & $0.4091(5)$ & 0.3351 & $0.8456(4)$ & $0.041(2)$ \\
\hline $\mathrm{H} 28$ & 0.341998 & 0.308064 & 0.817616 & $0.0498^{*}$ \\
\hline $\mathrm{C} 29$ & $0.5156(6)$ & 0.3004 & $0.8924(4)$ & $0.041(2)$ \\
\hline $\mathrm{C} 30$ & $0.6177(5)$ & 0.3387 & $0.9323(4)$ & $0.043(2)$ \\
\hline H30 & 0.687051 & 0.314123 & 0.961804 & $0.0515^{*}$ \\
\hline
\end{tabular}




$\begin{array}{lllll}\text { C31 } & 0.2956(6) & 0.4467(4) & 0.7881(5) & 0.047(3) \\ \text { N1 } & 0.4179(4) & 0.4525(2) & 0.5968(3) & 0.0338(17) \\ \text { N2 } & 0.2929(4) & 0.3229(2) & 0.5519(3) & 0.0387(18) \\ \text { N3 } & 0.0989(4) & 0.4627(2) & 0.3563(3) & 0.0413(18) \\ \text { N4 } & 0.1317(4) & 0.5603(2) & 0.5163(3) & 0.0405(18) \\ \text { N5 } & 0.7267(6) & 0.4510(4) & 0.9628(4) & 0.059(3) \\ \text { N6 } & 0.5168(6) & 0.2209(3) & 0.9002(4) & 0.055(3) \\ \text { O1 } & 0.7646(5) & 0.3117(3) & 0.7273(4) & 0.092(3) \\ \text { O2 } & 0.6406(5) & 0.1892(3) & 0.6987(4) & 0.102(3) \\ \text { O3 } & -0.0421(4) & 0.6544(2) & 0.1169(3) & 0.069(2) \\ \text { O4 } & -0.0501(5) & 0.7444(2) & 0.2776(4) & 0.081(2) \\ \text { O5 } & 0.8168(5) & 0.4145(3) & 0.9750(4) & 0.090(3) \\ \text { O6 } & 0.7298(5) & 0.5169(3) & 0.9762(4) & 0.084(2) \\ \text { O7 } & 0.5025(4) & 0.5233(2) & 0.8850(3) & 0.0617(19) \\ \text { O8 } & 0.2936(4) & 0.5168(3) & 0.7945(3) & 0.066(2) \\ \text { O9 } & 0.2137(4) & 0.4111(3) & 0.7370(3) & 0.064(2) \\ \text { O10 } & 0.4286(5) & 0.1877(2) & 0.8580(4) & 0.070(2) \\ \text { O11 } & 0.6096(5) & 0.1916(2) & 0.9512(4) & 0.068(2) \\ \text { H7 } & 0.388884 & 0.531747 & 0.83591 & 0.019(12)^{*}\end{array}$

Atomic displacement parameters $\left(\AA^{2}\right)$

\begin{tabular}{lllllll}
\hline & $U^{11}$ & $U^{22}$ & $U^{33}$ & $U^{12}$ & $U^{13}$ & $U^{23}$ \\
\hline $\mathrm{Ag} 1$ & $0.0474(4)$ & $0.0492(3)$ & $0.0603(3)$ & $0.0150(3)$ & $0.0038(2)$ & $0.0149(3)$ \\
$\mathrm{C} 1$ & $0.054(5)$ & $0.035(4)$ & $0.041(3)$ & $-0.006(3)$ & $0.014(3)$ & $0.001(3)$ \\
$\mathrm{C} 2$ & $0.052(5)$ & $0.041(4)$ & $0.048(4)$ & $-0.010(3)$ & $0.018(3)$ & $-0.001(3)$ \\
$\mathrm{C} 3$ & $0.031(4)$ & $0.063(4)$ & $0.043(3)$ & $-0.004(3)$ & $0.008(3)$ & $-0.008(3)$ \\
$\mathrm{C} 4$ & $0.036(4)$ & $0.034(3)$ & $0.035(3)$ & $0.015(3)$ & $0.013(3)$ & $0.004(3)$ \\
$\mathrm{C} 5$ & $0.085(7)$ & $0.078(6)$ & $0.048(4)$ & $0.014(5)$ & $0.031(4)$ & $0.006(4)$ \\
$\mathrm{C} 6$ & $0.201(12)$ & $0.037(5)$ & $0.065(5)$ & $0.012(6)$ & $0.081(7)$ & $0.001(4)$ \\
$\mathrm{C} 7$ & $0.033(4)$ & $0.059(5)$ & $0.035(3)$ & $0.007(3)$ & $0.008(3)$ & $0.001(3)$ \\
$\mathrm{C} 8$ & $0.088(6)$ & $0.023(3)$ & $0.051(4)$ & $0.010(4)$ & $0.029(4)$ & $0.005(3)$ \\
$\mathrm{C} 9$ & $0.074(6)$ & $0.053(5)$ & $0.067(5)$ & $-0.009(4)$ & $0.025(4)$ & $-0.008(4)$ \\
$\mathrm{C} 10$ & $0.039(4)$ & $0.054(4)$ & $0.064(4)$ & $-0.003(4)$ & $0.012(3)$ & $-0.014(4)$ \\
$\mathrm{C} 11$ & $0.043(4)$ & $0.026(3)$ & $0.029(3)$ & $0.006(3)$ & $0.010(3)$ & $0.002(2)$ \\
$\mathrm{C} 12$ & $0.043(4)$ & $0.031(3)$ & $0.028(3)$ & $-0.004(3)$ & $0.014(3)$ & $0.002(2)$ \\
$\mathrm{C} 13$ & $0.052(5)$ & $0.034(4)$ & $0.071(5)$ & $-0.002(3)$ & $0.005(4)$ & $-0.001(3)$ \\
$\mathrm{C} 14$ & $0.055(5)$ & $0.055(5)$ & $0.064(4)$ & $0.000(4)$ & $0.003(4)$ & $-0.024(4)$ \\
$\mathrm{C} 15$ & $0.046(4)$ & $0.057(4)$ & $0.045(4)$ & $-0.004(3)$ & $0.000(3)$ & $-0.002(3)$ \\
$\mathrm{C} 16$ & $0.029(3)$ & $0.038(3)$ & $0.051(4)$ & $0.000(3)$ & $0.004(3)$ & $0.005(3)$ \\
$\mathrm{C} 17$ & $0.039(4)$ & $0.054(4)$ & $0.055(4)$ & $0.002(3)$ & $0.001(3)$ & $0.021(4)$ \\
$\mathrm{C} 18$ & $0.036(4)$ & $0.042(4)$ & $0.077(5)$ & $-0.003(3)$ & $0.004(3)$ & $0.005(4)$ \\
$\mathrm{C} 19$ & $0.035(4)$ & $0.032(3)$ & $0.057(4)$ & $0.003(3)$ & $0.007(3)$ & $0.002(3)$ \\
$\mathrm{C} 20$ & $0.046(4)$ & $0.031(3)$ & $0.079(5)$ & $0.000(3)$ & $0.012(4)$ & $-0.010(4)$ \\
$\mathrm{C} 21$ & $0.049(5)$ & $0.055(5)$ & $0.067(5)$ & $0.002(4)$ & $0.010(4)$ & $-0.013(4)$ \\
$\mathrm{C} 22$ & $0.046(4)$ & $0.070(5)$ & $0.042(4)$ & $-0.003(4)$ & $0.001(3)$ & $-0.006(4)$ \\
$\mathrm{C} 23$ & $0.027(3)$ & $0.034(3)$ & $0.046(3)$ & $0.002(3)$ & $0.006(3)$ & $0.000(3)$ \\
$\mathrm{C} 24$ & $0.019(3)$ & $0.031(3)$ & $0.046(3)$ & $-0.001(2)$ & $0.007(2)$ & $0.004(3)$ \\
& & & & &
\end{tabular}




\begin{tabular}{lllllll} 
C25 & $0.058(5)$ & $0.037(4)$ & $0.028(3)$ & $-0.010(3)$ & $0.010(3)$ & $-0.004(3)$ \\
C26 & $0.062(5)$ & $0.032(3)$ & $0.030(3)$ & $0.001(3)$ & $0.018(3)$ & $-0.002(3)$ \\
C27 & $0.039(4)$ & $0.038(4)$ & $0.035(3)$ & $0.002(3)$ & $0.012(3)$ & $0.006(3)$ \\
C28 & $0.057(5)$ & $0.041(4)$ & $0.030(3)$ & $-0.007(3)$ & $0.018(3)$ & $-0.002(3)$ \\
C29 & $0.068(5)$ & $0.029(3)$ & $0.032(3)$ & $0.004(3)$ & $0.024(3)$ & $0.005(3)$ \\
C30 & $0.047(4)$ & $0.047(4)$ & $0.036(3)$ & $0.005(3)$ & $0.014(3)$ & $0.002(3)$ \\
C31 & $0.057(5)$ & $0.041(4)$ & $0.048(4)$ & $0.011(4)$ & $0.023(3)$ & $0.008(3)$ \\
N1 & $0.036(3)$ & $0.031(3)$ & $0.033(2)$ & $0.005(2)$ & $0.008(2)$ & $0.001(2)$ \\
N2 & $0.033(3)$ & $0.037(3)$ & $0.044(3)$ & $-0.001(2)$ & $0.006(2)$ & $-0.002(2)$ \\
N3 & $0.040(3)$ & $0.029(3)$ & $0.051(3)$ & $0.001(2)$ & $0.004(2)$ & $-0.003(2)$ \\
N4 & $0.035(3)$ & $0.043(3)$ & $0.041(3)$ & $0.003(2)$ & $0.004(2)$ & $0.006(2)$ \\
N5 & $0.057(4)$ & $0.072(5)$ & $0.044(3)$ & $-0.015(4)$ & $0.003(3)$ & $-0.009(3)$ \\
N6 & $0.090(5)$ & $0.039(4)$ & $0.044(3)$ & $0.004(3)$ & $0.033(3)$ & $0.004(3)$ \\
O1 & $0.083(4)$ & $0.104(4)$ & $0.088(4)$ & $0.034(4)$ & $0.022(3)$ & $0.021(3)$ \\
O2 & $0.095(5)$ & $0.085(4)$ & $0.115(5)$ & $0.014(4)$ & $0.002(4)$ & $-0.007(4)$ \\
O3 & $0.066(3)$ & $0.071(3)$ & $0.065(3)$ & $0.002(3)$ & $0.004(3)$ & $0.026(3)$ \\
O4 & $0.101(4)$ & $0.040(3)$ & $0.095(4)$ & $0.019(3)$ & $0.007(3)$ & $0.017(3)$ \\
O5 & $0.052(4)$ & $0.100(4)$ & $0.111(4)$ & $-0.002(3)$ & $0.006(3)$ & $-0.031(3)$ \\
O6 & $0.091(4)$ & $0.051(3)$ & $0.090(4)$ & $-0.024(3)$ & $-0.015(3)$ & $0.003(3)$ \\
O7 & $0.088(4)$ & $0.042(3)$ & $0.057(3)$ & $-0.005(2)$ & $0.021(3)$ & $-0.002(2)$ \\
O8 & $0.069(4)$ & $0.060(3)$ & $0.072(3)$ & $0.017(3)$ & $0.022(3)$ & $0.009(2)$ \\
O9 & $0.042(3)$ & $0.084(4)$ & $0.066(3)$ & $0.006(3)$ & $0.012(2)$ & $-0.002(3)$ \\
O10 & $0.107(5)$ & $0.039(3)$ & $0.067(3)$ & $-0.016(3)$ & $0.025(3)$ & $-0.001(2)$ \\
O11 & $0.089(4)$ & $0.042(3)$ & $0.081(3)$ & $0.016(3)$ & $0.036(3)$ & $0.022(2)$ \\
& & & & & \\
\hline
\end{tabular}

Geometric parameters $\left(\AA,{ }^{\circ}\right)$

\begin{tabular}{llll}
\hline $\mathrm{Ag} 1-\mathrm{N} 1$ & $2.404(4)$ & $\mathrm{C} 16-\mathrm{C} 24$ & $1.392(7)$ \\
$\mathrm{Ag} 1-\mathrm{N} 2$ & $2.348(5)$ & $\mathrm{C} 17-\mathrm{C} 18$ & $1.476(9)$ \\
$\mathrm{Ag} 1-\mathrm{N} 3$ & $2.335(4)$ & $\mathrm{C} 17-\mathrm{O} 3$ & $1.220(8)$ \\
$\mathrm{Ag} 1-\mathrm{N} 4$ & $2.376(5)$ & $\mathrm{C} 18-\mathrm{C} 19$ & $1.498(8)$ \\
$\mathrm{C} 1-\mathrm{H} 1$ & 0.93 & $\mathrm{C} 18-\mathrm{O} 4$ & $1.227(8)$ \\
$\mathrm{C} 1-\mathrm{C} 2$ & $1.376(8)$ & $\mathrm{C} 19-\mathrm{C} 20$ & $1.386(8)$ \\
$\mathrm{C} 1-\mathrm{N} 1$ & $1.334(7)$ & $\mathrm{C} 19-\mathrm{C} 23$ & $1.400(8)$ \\
$\mathrm{C} 2-\mathrm{H} 2$ & 0.93 & $\mathrm{C} 20-\mathrm{H} 20$ & 0.93 \\
$\mathrm{C} 2-\mathrm{C} 3$ & $1.373(8)$ & $\mathrm{C} 20-\mathrm{C} 21$ & $1.359(9)$ \\
$\mathrm{C} 3-\mathrm{H} 3$ & 0.93 & $\mathrm{C} 21-\mathrm{H} 21$ & 0.93 \\
$\mathrm{C} 3-\mathrm{C} 4$ & $1.372(8)$ & $\mathrm{C} 21-\mathrm{C} 22$ & $1.373(9)$ \\
$\mathrm{C} 4-\mathrm{C} 5$ & $1.475(10)$ & $\mathrm{C} 22-\mathrm{H} 22$ & 0.93 \\
$\mathrm{C} 4-\mathrm{C} 12$ & $1.402(8)$ & $\mathrm{C} 22-\mathrm{N} 4$ & $1.341(8)$ \\
$\mathrm{C} 5-\mathrm{C} 6$ & $1.349(12)$ & $\mathrm{C} 23-\mathrm{C} 24$ & $1.478(7)$ \\
$\mathrm{C} 5-\mathrm{O} 1$ & $1.298(10)$ & $\mathrm{C} 23-\mathrm{N} 4$ & $1.349(7)$ \\
$\mathrm{C} 6-\mathrm{C} 7$ & $1.493(13)$ & $\mathrm{C} 24-\mathrm{N} 3$ & $1.344(7)$ \\
$\mathrm{C} 6-\mathrm{O} 2$ & $1.283(10)$ & $\mathrm{C} 25-\mathrm{C} 26$ & $1.430(9)$ \\
$\mathrm{C} 7-\mathrm{C} 8$ & $1.399(9)$ & $\mathrm{C} 25-\mathrm{C} 30$ & $1.384(8)$ \\
$\mathrm{C} 7-\mathrm{C} 11$ & $1.385(8)$ & $\mathrm{C} 25-\mathrm{N} 5$ & $1.450(9)$ \\
$\mathrm{C} 8-\mathrm{H} 8$ & 0.93 & $\mathrm{C} 26-\mathrm{C} 27$ & $1.418(8)$ \\
$\mathrm{C} 8-\mathrm{C} 9$ & $1.357(10)$ & $\mathrm{C} 26-\mathrm{O} 7$ & $1.293(7)$
\end{tabular}




\begin{tabular}{|c|c|c|c|}
\hline C9- $\mathrm{H} 9$ & 0.93 & $\mathrm{C} 27-\mathrm{C} 28$ & $1.373(8)$ \\
\hline $\mathrm{C} 9-\mathrm{C} 10$ & $1.353(9)$ & $\mathrm{C} 27-\mathrm{C} 31$ & $1.496(8)$ \\
\hline $\mathrm{C} 10-\mathrm{H} 10$ & 0.93 & $\mathrm{C} 28-\mathrm{H} 28$ & 0.93 \\
\hline $\mathrm{C} 10-\mathrm{N} 2$ & $1.347(8)$ & $\mathrm{C} 28-\mathrm{C} 29$ & $1.407(8)$ \\
\hline $\mathrm{C} 11-\mathrm{C} 12$ & $1.481(7)$ & $\mathrm{C} 29-\mathrm{C} 30$ & $1.380(8)$ \\
\hline $\mathrm{C} 11-\mathrm{N} 2$ & $1.326(7)$ & $\mathrm{C} 29-\mathrm{N} 6$ & $1.458(8)$ \\
\hline $\mathrm{C} 12-\mathrm{N} 1$ & $1.344(7)$ & $\mathrm{C} 30-\mathrm{H} 30$ & 0.93 \\
\hline $\mathrm{C} 13-\mathrm{H} 13$ & 0.93 & $\mathrm{C} 31-\mathrm{O} 8$ & $1.286(8)$ \\
\hline $\mathrm{C} 13-\mathrm{C} 14$ & $1.381(9)$ & $\mathrm{C} 31-\mathrm{O} 9$ & $1.223(8)$ \\
\hline $\mathrm{C} 13-\mathrm{N} 3$ & $1.339(8)$ & $\mathrm{N} 5-\mathrm{O} 5$ & $1.230(9)$ \\
\hline $\mathrm{C} 14-\mathrm{H} 14$ & 0.93 & $\mathrm{~N} 5-\mathrm{O} 6$ & $1.218(8)$ \\
\hline $\mathrm{C} 14-\mathrm{C} 15$ & $1.373(9)$ & $\mathrm{N} 6-\mathrm{O} 10$ & $1.216(8)$ \\
\hline $\mathrm{C} 15-\mathrm{H} 15$ & 0.93 & $\mathrm{~N} 6-\mathrm{O} 11$ & $1.257(8)$ \\
\hline $\mathrm{C} 15-\mathrm{C} 16$ & $1.396(8)$ & $\mathrm{O} 7-\mathrm{H} 7$ & $1.346(4)$ \\
\hline $\mathrm{C} 16-\mathrm{C} 17$ & $1.473(8)$ & $\mathrm{O} 8-\mathrm{H} 7$ & 1.155 (4) \\
\hline $\mathrm{H} 1-\mathrm{C} 1-\mathrm{C} 2$ & 118.04 & $\mathrm{C} 19-\mathrm{C} 18-\mathrm{O} 4$ & $120.9(6)$ \\
\hline $\mathrm{H} 1-\mathrm{C} 1-\mathrm{N} 1$ & 118.04 & $\mathrm{C} 18-\mathrm{C} 19-\mathrm{C} 20$ & $121.6(5)$ \\
\hline $\mathrm{C} 2-\mathrm{C} 1-\mathrm{N} 1$ & $123.9(5)$ & $\mathrm{C} 18-\mathrm{C} 19-\mathrm{C} 23$ & $119.3(5)$ \\
\hline $\mathrm{C} 1-\mathrm{C} 2-\mathrm{H} 2$ & 120.88 & $\mathrm{C} 20-\mathrm{C} 19-\mathrm{C} 23$ & $119.1(5)$ \\
\hline $\mathrm{C} 1-\mathrm{C} 2-\mathrm{C} 3$ & $118.2(5)$ & $\mathrm{C} 19-\mathrm{C} 20-\mathrm{H} 20$ & 119.84 \\
\hline $\mathrm{H} 2-\mathrm{C} 2-\mathrm{C} 3$ & 120.88 & $\mathrm{C} 19-\mathrm{C} 20-\mathrm{C} 21$ & $120.3(6)$ \\
\hline $\mathrm{C} 2-\mathrm{C} 3-\mathrm{H} 3$ & 120.34 & $\mathrm{H} 20-\mathrm{C} 20-\mathrm{C} 21$ & 119.84 \\
\hline $\mathrm{C} 2-\mathrm{C} 3-\mathrm{C} 4$ & $119.3(5)$ & $\mathrm{C} 20-\mathrm{C} 21-\mathrm{H} 21$ & 121.42 \\
\hline $\mathrm{H} 3-\mathrm{C} 3-\mathrm{C} 4$ & 120.34 & $\mathrm{C} 20-\mathrm{C} 21-\mathrm{C} 22$ & $117.2(6)$ \\
\hline $\mathrm{C} 3-\mathrm{C} 4-\mathrm{C} 5$ & $121.7(6)$ & $\mathrm{H} 21-\mathrm{C} 21-\mathrm{C} 22$ & 121.42 \\
\hline $\mathrm{C} 3-\mathrm{C} 4-\mathrm{C} 12$ & $119.3(5)$ & $\mathrm{C} 21-\mathrm{C} 22-\mathrm{H} 22$ & 117.46 \\
\hline $\mathrm{C} 5-\mathrm{C} 4-\mathrm{C} 12$ & $119.0(5)$ & $\mathrm{C} 21-\mathrm{C} 22-\mathrm{N} 4$ & $125.1(5)$ \\
\hline $\mathrm{C} 4-\mathrm{C} 5-\mathrm{C} 6$ & $121.9(8)$ & $\mathrm{H} 22-\mathrm{C} 22-\mathrm{N} 4$ & 117.46 \\
\hline $\mathrm{C} 4-\mathrm{C} 5-\mathrm{O} 1$ & $118.9(7)$ & $\mathrm{C} 19-\mathrm{C} 23-\mathrm{C} 24$ & $121.2(5)$ \\
\hline $\mathrm{C} 6-\mathrm{C} 5-\mathrm{O} 1$ & $119.2(8)$ & $\mathrm{C} 19-\mathrm{C} 23-\mathrm{N} 4$ & $120.8(5)$ \\
\hline $\mathrm{C} 5-\mathrm{C} 6-\mathrm{C} 7$ & $119.3(7)$ & $\mathrm{C} 24-\mathrm{C} 23-\mathrm{N} 4$ & $118.0(5)$ \\
\hline $\mathrm{C} 5-\mathrm{C} 6-\mathrm{O} 2$ & $121.4(9)$ & $\mathrm{C} 16-\mathrm{C} 24-\mathrm{C} 23$ & $120.2(5)$ \\
\hline $\mathrm{C} 7-\mathrm{C} 6-\mathrm{O} 2$ & $119.3(7)$ & $\mathrm{C} 16-\mathrm{C} 24-\mathrm{N} 3$ & $122.5(5)$ \\
\hline $\mathrm{C} 6-\mathrm{C} 7-\mathrm{C} 8$ & $119.6(6)$ & $\mathrm{C} 23-\mathrm{C} 24-\mathrm{N} 3$ & $117.2(4)$ \\
\hline $\mathrm{C} 6-\mathrm{C} 7-\mathrm{C} 11$ & $120.4(6)$ & $\mathrm{C} 26-\mathrm{C} 25-\mathrm{C} 30$ & $121.2(5)$ \\
\hline $\mathrm{C} 8-\mathrm{C} 7-\mathrm{C} 11$ & $120.0(6)$ & $\mathrm{C} 26-\mathrm{C} 25-\mathrm{N} 5$ & $122.7(5)$ \\
\hline $\mathrm{C} 7-\mathrm{C} 8-\mathrm{H} 8$ & 121.23 & $\mathrm{C} 30-\mathrm{C} 25-\mathrm{N} 5$ & $116.1(6)$ \\
\hline $\mathrm{C} 7-\mathrm{C} 8-\mathrm{C} 9$ & $117.5(6)$ & $\mathrm{C} 25-\mathrm{C} 26-\mathrm{C} 27$ & $117.1(5)$ \\
\hline $\mathrm{H} 8-\mathrm{C} 8-\mathrm{C} 9$ & 121.23 & $\mathrm{C} 25-\mathrm{C} 26-\mathrm{O} 7$ & $122.2(5)$ \\
\hline $\mathrm{C} 8-\mathrm{C} 9-\mathrm{H} 9$ & 120.26 & $\mathrm{C} 27-\mathrm{C} 26-\mathrm{O} 7$ & $120.5(5)$ \\
\hline $\mathrm{C} 8-\mathrm{C} 9-\mathrm{C} 10$ & $119.5(6)$ & $\mathrm{C} 26-\mathrm{C} 27-\mathrm{C} 28$ & $122.1(5)$ \\
\hline $\mathrm{H} 9-\mathrm{C} 9-\mathrm{C} 10$ & 120.26 & $\mathrm{C} 26-\mathrm{C} 27-\mathrm{C} 31$ & $120.1(5)$ \\
\hline $\mathrm{C} 9-\mathrm{C} 10-\mathrm{H} 10$ & 118.01 & $\mathrm{C} 28-\mathrm{C} 27-\mathrm{C} 31$ & $117.8(5)$ \\
\hline $\mathrm{C} 9-\mathrm{C} 10-\mathrm{N} 2$ & $124.0(6)$ & $\mathrm{C} 27-\mathrm{C} 28-\mathrm{H} 28$ & 121 \\
\hline $\mathrm{H} 10-\mathrm{C} 10-\mathrm{N} 2$ & 118.01 & $\mathrm{C} 27-\mathrm{C} 28-\mathrm{C} 29$ & $118.0(5)$ \\
\hline $\mathrm{C} 7-\mathrm{C} 11-\mathrm{C} 12$ & $119.5(5)$ & $\mathrm{H} 28-\mathrm{C} 28-\mathrm{C} 29$ & 121 \\
\hline $\mathrm{C} 7-\mathrm{C} 11-\mathrm{N} 2$ & $121.3(5)$ & $\mathrm{C} 28-\mathrm{C} 29-\mathrm{C} 30$ & $122.6(5)$ \\
\hline
\end{tabular}




$\begin{array}{llll}\mathrm{C} 12-\mathrm{C} 11-\mathrm{N} 2 & 119.2(5) & \mathrm{C} 28-\mathrm{C} 29-\mathrm{N} 6 & 118.3(5) \\ \mathrm{C} 4-\mathrm{C} 12-\mathrm{C} 11 & 119.8(5) & \mathrm{C} 30-\mathrm{C} 29-\mathrm{N} 6 & 119.1(5) \\ \mathrm{C} 4-\mathrm{C} 12-\mathrm{N} 1 & 121.2(5) & \mathrm{C} 25-\mathrm{C} 30-\mathrm{C} 29 & 118.7(5) \\ \mathrm{C} 11-\mathrm{C} 12-\mathrm{N} 1 & 118.9(5) & \mathrm{C} 25-\mathrm{C} 30-\mathrm{H} 30 & 120.65 \\ \mathrm{H} 13-\mathrm{C} 13-\mathrm{C} 14 & 117.83 & \mathrm{C} 29-\mathrm{C} 30-\mathrm{H} 30 & 120.65 \\ \mathrm{H} 13-\mathrm{C} 13-\mathrm{N} 3 & \mathrm{C} 27-\mathrm{C} 31-\mathrm{O} 8 & 116.2(5) \\ \mathrm{C} 14-\mathrm{C} 13-\mathrm{N} 3 & 117.83 & \mathrm{C} 27-\mathrm{C} 31-\mathrm{O} 9 & 120.8(6) \\ \mathrm{C} 13-\mathrm{C} 14-\mathrm{H} 14 & 124.3(5) & \mathrm{O}-\mathrm{C} 31-\mathrm{O} 9 & 122.9(6) \\ \mathrm{C} 13-\mathrm{C} 14-\mathrm{C} 15 & 121.04 & \mathrm{C} 1-\mathrm{N} 1-\mathrm{C} 12 & 117.9(4) \\ \mathrm{H} 14-\mathrm{C} 14-\mathrm{C} 15 & 117.9(6) & \mathrm{C} 10-\mathrm{N} 2-\mathrm{C} 11 & 117.6(5) \\ \mathrm{C} 14-\mathrm{C} 15-\mathrm{H} 15 & 121.04 & \mathrm{C} 13-\mathrm{N} 3-\mathrm{C} 24 & 117.5(5) \\ \mathrm{C} 14-\mathrm{C} 15-\mathrm{C} 16 & 120.29 & \mathrm{C} 25-\mathrm{N} 4-\mathrm{N} 5-\mathrm{O} 23 & 118.4(6) \\ \mathrm{H} 15-\mathrm{C} 15-\mathrm{C} 16 & 119.4(5) & \mathrm{C} 25-\mathrm{N} 5-\mathrm{O} 6 & 120.0(6) \\ \mathrm{C} 15-\mathrm{C} 16-\mathrm{C} 17 & 120.29 & \mathrm{O}-\mathrm{N} 5-\mathrm{O} 6 & 118.3(6) \\ \mathrm{C} 15-\mathrm{C} 16-\mathrm{C} 24 & 120.1(5) & \mathrm{C} 29-\mathrm{N} 6-\mathrm{O} 10 & 117.2(5) \\ \mathrm{C} 17-\mathrm{C} 16-\mathrm{C} 24 & 118.5(5) & \mathrm{C} 29-\mathrm{N} 6-\mathrm{O} 11 & 124.5(5) \\ \mathrm{C} 16-\mathrm{C} 17-\mathrm{C} 18 & 121.4(5) & \mathrm{O} 10-\mathrm{N} 6-\mathrm{O} 11 & 99.4(4) \\ \mathrm{C} 16-\mathrm{C} 17-\mathrm{O} 3 & 118.1(5) & \mathrm{C} 26-\mathrm{O} 7-\mathrm{H} 7 & 103.7(4) \\ \mathrm{C} 18-\mathrm{C} 17-\mathrm{O} 3 & 122.0(6) & \mathrm{O} 7-\mathrm{H} 7-\mathrm{O} 8 & 159.6(3) \\ \mathrm{C} 17-\mathrm{C} 18-\mathrm{C} 19 & 119.9(6) & & \end{array}$

Hydrogen-bond geometry $\left(A,{ }^{\circ}\right)$

\begin{tabular}{lllll}
\hline$D-\mathrm{H} \cdots A$ & $D-\mathrm{H}$ & $\mathrm{H} \cdots A$ & $D \cdots A$ & $D-\mathrm{H} \cdots A$ \\
\hline $\mathrm{C} 2-\mathrm{H} 2 \cdots \mathrm{O} 10^{\mathrm{i}}$ & 0.93 & 2.49 & $3.180(7)$ & 131 \\
$\mathrm{C} 8-\mathrm{H} 8 \cdots \mathrm{O}^{\mathrm{ii}}$ & 0.93 & 2.32 & $3.220(7)$ & 162 \\
$\mathrm{C} 9-\mathrm{H} 9 \cdots$ O6 $^{\mathrm{ii}}$ & 0.93 & 2.49 & $3.243(9)$ & 138 \\
$\mathrm{C} 13-\mathrm{H} 13 \cdots \mathrm{O} 4^{\mathrm{iii}}$ & 0.93 & 2.47 & $3.209(7)$ & 137 \\
$\mathrm{C} 22-\mathrm{H} 22 \cdots \mathrm{O} 8$ & 0.93 & 2.36 & $3.251(7)$ & 160 \\
$\mathrm{O} 7-\mathrm{H} 7 \cdots \mathrm{C} 31$ & $1.346(4)$ & $1.922(7)$ & $2.833(8)$ & $119.1(3)$ \\
$\mathrm{O} 7-\mathrm{H} 7 \cdots \mathrm{O} 8$ & $1.346(4)$ & $1.155(4)$ & $2.462(6)$ & $159.6(3)$ \\
$\mathrm{O} 8-\mathrm{H} 7 \cdots \mathrm{C} 26$ & $1.155(4)$ & $2.013(6)$ & $2.781(7)$ & $120.3(3)$ \\
$\mathrm{O} 8-\mathrm{H} 7 \cdots \mathrm{O} 7$ & $1.155(4)$ & $1.346(4)$ & $2.462(6)$ & $159.6(3)$
\end{tabular}

Symmetry codes: (i) $-x+1, y+1 / 2,-z+3 / 2$; (ii) $-x+1, y-1 / 2,-z+3 / 2$; (iii) $-x, y-1 / 2,-z+1 / 2$.

3,5-Dimethylpyrazolium 3,5-dinitrosalicylate (SEDKET)

Crystal data

$\mathrm{C}_{5} \mathrm{H}_{9} \mathrm{~N}_{2}{ }^{+} \cdot \mathrm{C}_{7} \mathrm{H}_{3} \mathrm{~N}_{2} \mathrm{O}_{7}$

$M_{r}=324.26$

Monoclinic, $P 2_{1}$

$a=8.1183(7) \AA$

$b=6.0636(5) \AA$

$c=14.1453(11) \AA$

$\beta=91.904(1)^{\circ}$

$V=695.93(10) \AA^{3}$

$Z=2$
$F(000)=336$

$D_{\mathrm{x}}=1.547 \mathrm{Mg} \mathrm{m}^{-3}$

Mo $K \alpha$ radiation, $\lambda=0.71073 \AA$

Cell parameters from 1025 reflections

$\theta=2.5-22.6^{\circ}$

$\mu=0.13 \mathrm{~mm}^{-1}$

$T=293 \mathrm{~K}$

Block, colorless

$0.40 \times 0.27 \times 0.11 \mathrm{~mm}$ 


\section{Data collection}

Bruker SMART CCD

diffractometer

Radiation source: fine-focus sealed tube

Graphite monochromator

$\omega$ scans

Absorption correction: multi-scan

(SADABS; Bruker, 2002)

$T_{\min }=0.959, T_{\max }=0.986$

\section{Refinement}

Refinement on $F^{2}$

Least-squares matrix: full

$R[F>3 \sigma(F)]=0.041$

$w R(F)=0.088$

$S=1.16$

2301 reflections

212 parameters

0 restraints

37 constraints

Primary atom site location: structure-invariant direct methods

Secondary atom site location: difference Fourier map

Hydrogen site location: difference Fourier map
3523 measured reflections

2301 independent reflections

1444 reflections with $I>3 \sigma(I)$

$R_{\text {int }}=0.040$

$\theta_{\max }=25.0^{\circ}, \theta_{\min }=2.5^{\circ}$

$h=-9 \rightarrow 9$

$k=-7 \rightarrow 7$

$l=-16 \rightarrow 12$

$\mathrm{H}$ atoms treated by a mixture of independent and constrained refinement

Weighting scheme based on measured s.u.'s $w=$ $1 /\left(\sigma^{2}(I)+0.0004 I^{2}\right)$

$(\Delta / \sigma)_{\max }=0.033$

$\Delta \rho_{\max }=0.11$ e $\AA^{-3}$

$\Delta \rho_{\text {min }}=-0.10$ e $\AA^{-3}$

Extinction correction: B-C type 1 Lorentzian isotropic (Becker \& Coppens, 1974)

Extinction coefficient: 3100 (400)

Absolute structure: 955 of Friedel pairs used in the refinement

Absolute structure parameter: 0.5

\section{Special details}

Geometry. All e.s.d.'s (except the e.s.d. in the dihedral angle between two 1.s. planes) are estimated using the full covariance matrix. The cell e.s.d.'s are taken into account individually in the estimation of e.s.d.'s in distances, angles and torsion angles; correlations between e.s.d.'s in cell parameters are only used when they are defined by crystal symmetry. An approximate (isotropic) treatment of cell e.s.d.'s is used for estimating e.s.d.'s involving 1.s. planes.

Refinement. Refinement of $F^{2}$ against ALL reflections. The weighted $R$-factor $w R$ and goodness of fit $S$ are based on $F^{2}$, conventional $R$-factors $R$ are based on $F$, with $F$ set to zero for negative $F^{2}$. The threshold expression of $F^{2}>\sigma\left(F^{2}\right)$ is used only for calculating $R$-factors(gt) etc. and is not relevant to the choice of reflections for refinement. $R$-factors based on $F^{2}$ are statistically about twice as large as those based on $F$, and $R$ - factors based on ALL data will be even larger.

Number of fixed parameters 10 .

Fractional atomic coordinates and isotropic or equivalent isotropic displacement parameters $\left(\AA^{2}\right)$

\begin{tabular}{lllll}
\hline & $x$ & $y$ & $z$ & $U_{\text {iso }} * / U_{\text {eq }}$ \\
\hline $\mathrm{N} 1$ & $0.2262(4)$ & $0.7164(5)$ & $0.2338(2)$ & $0.0464(11)$ \\
$\mathrm{N} 2$ & $0.2203(3)$ & $0.6196(5)$ & $0.3198(2)$ & $0.0478(11)$ \\
$\mathrm{C} 6$ & $0.3519(4)$ & $0.7226(6)$ & $0.5525(2)$ & $0.0412(12)$ \\
$\mathrm{N} 3$ & $0.2179(3)$ & $1.3158(5)$ & $0.7705(2)$ & $0.0509(12)$ \\
$\mathrm{N} 4$ & $0.5552(4)$ & $0.7120(5)$ & $0.8913(2)$ & $0.0501(12)$ \\
O1 & $0.5417(3)$ & $0.5285(4)$ & $0.70239(16)$ & $0.0462(9)$ \\
O2 & $0.4266(3)$ & $0.5357(4)$ & $0.53811(15)$ & $0.0582(10)$ \\
O3 & $0.2657(3)$ & $0.8100(4)$ & $0.49006(14)$ & $0.0507(8)$ \\
O4 & $0.1415(3)$ & $1.4013(4)$ & $0.70419(18)$ & $0.0652(10)$ \\
O5 & $0.2270(3)$ & $1.3968(4)$ & $0.85025(18)$ & $0.0709(11)$ \\
O6 & $0.5263(4)$ & $0.7839(5)$ & $0.96960(17)$ & $0.0853(12)$ \\
O7 & $0.6549(4)$ & $0.5664(5)$ & $0.87980(17)$ & $0.0719(12)$
\end{tabular}




$\begin{array}{lllll}\text { C1 } & 0.1300(5) & 0.6431(8) & 0.0685(2) & 0.0762(19) \\ \text { H1a } & 0.132119 & 0.800298 & 0.060733 & 0.1144^{*} \\ \text { H1b } & 0.027525 & 0.586015 & 0.043068 & 0.1144^{*} \\ \text { H1c } & 0.219699 & 0.578396 & 0.035744 & 0.1144^{*} \\ \text { C2 } & 0.1466(4) & 0.5878(6) & 0.1713(2) & 0.0469(13) \\ \text { C3 } & 0.0892(4) & 0.4058(7) & 0.2187(3) & 0.0553(14) \\ \text { H3 } & 0.029651 & 0.288588 & 0.192446 & 0.0663^{*} \\ \text { C4 } & 0.1373(4) & 0.4313(6) & 0.3133(2) & 0.0481(13) \\ \text { C5 } & 0.1093(5) & 0.2896(7) & 0.3966(3) & 0.0669(16) \\ \text { H5a } & 0.025142 & 0.183251 & 0.380914 & 0.1003^{*} \\ \text { H5b } & 0.20964 & 0.214551 & 0.414477 & 0.1003^{*} \\ \text { H5c } & 0.074882 & 0.379302 & 0.448238 & 0.1003^{*} \\ \text { C12 } & 0.4691(4) & 0.7102(6) & 0.7196(2) & 0.0372(12) \\ \text { C7 } & 0.3736(3) & 0.8233(6) & 0.6477(2) & 0.0348(11) \\ \text { C8 } & 0.2972(4) & 1.0181(6) & 0.6641(2) & 0.0380(12) \\ \text { H8 } & 0.239805 & 1.089516 & 0.615013 & 0.0456^{*} \\ \text { C9 } & 0.3044(4) & 1.1108(6) & 0.7534(2) & 0.0381(11) \\ \text { C10 } & 0.3902(4) & 1.0091(6) & 0.8274(2) & 0.0403(12) \\ \text { H10 } & 0.393751 & 1.071121 & 0.887603 & 0.0483^{*} \\ \text { C11 } & 0.4704(4) & 0.8133(6) & 0.8100(2) & 0.0380(11) \\ \text { H2a } & 0.507(5) & 0.485(9) & 0.620(3) & 0.145(19)^{*} \\ \text { H1 } & 0.289(6) & 0.878(9) & 0.225(3) & 0.130(19)^{*} \\ \text { H2 } & 0.258(4) & 0.707(6) & 0.373(2) & 0.063(12)^{*}\end{array}$

Atomic displacement parameters $\left(\AA^{2}\right)$

\begin{tabular}{lllllll}
\hline & $U^{11}$ & $U^{22}$ & $U^{33}$ & $U^{12}$ & $U^{13}$ & $U^{23}$ \\
\hline $\mathrm{N} 1$ & $0.0502(18)$ & $0.049(2)$ & $0.0399(18)$ & $0.0006(15)$ & $-0.0027(14)$ & $-0.0034(15)$ \\
$\mathrm{N} 2$ & $0.0497(19)$ & $0.051(2)$ & $0.0422(18)$ & $-0.0046(17)$ & $-0.0047(14)$ & $-0.0066(17)$ \\
$\mathrm{C} 6$ & $0.0393(19)$ & $0.047(2)$ & $0.037(2)$ & $-0.0016(18)$ & $0.0053(16)$ & $-0.0027(18)$ \\
$\mathrm{N} 3$ & $0.0500(19)$ & $0.043(2)$ & $0.060(2)$ & $-0.0003(17)$ & $0.0048(16)$ & $-0.0031(18)$ \\
$\mathrm{N} 4$ & $0.065(2)$ & $0.046(2)$ & $0.0387(19)$ & $0.0015(17)$ & $-0.0037(16)$ & $0.0042(16)$ \\
O1 & $0.0564(15)$ & $0.0414(15)$ & $0.0407(14)$ & $0.0091(13)$ & $-0.0027(11)$ & $-0.0072(12)$ \\
O2 & $0.0704(17)$ & $0.0626(18)$ & $0.0414(14)$ & $0.0205(15)$ & $-0.0036(12)$ & $-0.0156(14)$ \\
O3 & $0.0581(14)$ & $0.0600(17)$ & $0.0333(13)$ & $0.0050(14)$ & $-0.0083(11)$ & $0.0013(12)$ \\
O4 & $0.0703(17)$ & $0.0521(17)$ & $0.0721(18)$ & $0.0185(15)$ & $-0.0141(14)$ & $0.0031(15)$ \\
O5 & $0.092(2)$ & $0.0593(19)$ & $0.0617(17)$ & $0.0072(16)$ & $0.0048(14)$ & $-0.0187(15)$ \\
O6 & $0.148(3)$ & $0.075(2)$ & $0.0326(16)$ & $0.035(2)$ & $-0.0069(15)$ & $-0.0032(15)$ \\
O7 & $0.092(2)$ & $0.072(2)$ & $0.0510(16)$ & $0.0308(19)$ & $-0.0083(14)$ & $0.0090(16)$ \\
C1 & $0.086(3)$ & $0.096(4)$ & $0.047(2)$ & $-0.014(3)$ & $-0.009(2)$ & $-0.007(2)$ \\
C2 & $0.043(2)$ & $0.054(3)$ & $0.0434(19)$ & $0.005(2)$ & $-0.0046(17)$ & $-0.009(2)$ \\
C3 & $0.049(2)$ & $0.057(3)$ & $0.060(2)$ & $-0.004(2)$ & $-0.0075(18)$ & $-0.022(2)$ \\
C4 & $0.039(2)$ & $0.044(2)$ & $0.062(2)$ & $0.0027(19)$ & $0.0037(17)$ & $-0.003(2)$ \\
C5 & $0.066(3)$ & $0.060(3)$ & $0.074(3)$ & $0.000(2)$ & $-0.002(2)$ & $0.012(2)$ \\
C12 & $0.0351(19)$ & $0.041(2)$ & $0.036(2)$ & $-0.0079(18)$ & $0.0025(15)$ & $0.0010(17)$ \\
C7 & $0.0342(17)$ & $0.039(2)$ & $0.0316(17)$ & $-0.0052(17)$ & $0.0004(13)$ & $0.0031(16)$ \\
C8 & $0.041(2)$ & $0.038(2)$ & $0.0349(19)$ & $-0.0032(18)$ & $-0.0036(15)$ & $0.0039(17)$ \\
C9 & $0.041(2)$ & $0.032(2)$ & $0.0416(19)$ & $-0.0045(17)$ & $0.0020(15)$ & $-0.0015(17)$
\end{tabular}




\begin{tabular}{lllllll}
$\mathrm{C} 10$ & $0.049(2)$ & $0.039(2)$ & $0.0325(19)$ & $-0.0038(18)$ & $0.0020(16)$ & $-0.0017(17)$ \\
$\mathrm{C} 11$ & $0.0419(19)$ & $0.041(2)$ & $0.0312(17)$ & $-0.0038(19)$ & $-0.0025(14)$ & $0.0049(17)$ \\
\hline
\end{tabular}

Geometric parameters $(\AA, \stackrel{o}{)}$

\begin{tabular}{|c|c|c|c|}
\hline $\mathrm{N} 1-\mathrm{N} 2$ & $1.353(4)$ & $\mathrm{H} 1 \mathrm{a}-\mathrm{H} 1 \mathrm{~b}$ & 1.5677 \\
\hline $\mathrm{N} 1-\mathrm{C} 2$ & $1.330(5)$ & $\mathrm{H} 1 \mathrm{a}-\mathrm{H} 1 \mathrm{c}$ & 1.5677 \\
\hline $\mathrm{N} 1-\mathrm{H} 1$ & $1.11(5)$ & $\mathrm{H} 1 \mathrm{~b}-\mathrm{H} 1 \mathrm{c}$ & 1.5677 \\
\hline $\mathrm{N} 2-\mathrm{C} 4$ & $1.327(5)$ & $\mathrm{C} 2-\mathrm{C} 3$ & $1.380(5)$ \\
\hline $\mathrm{N} 2-\mathrm{H} 2$ & $0.96(3)$ & $\mathrm{C} 3-\mathrm{H} 3$ & 0.93 \\
\hline $\mathrm{C} 6-\mathrm{O} 2$ & $1.304(4)$ & $\mathrm{C} 3-\mathrm{C} 4$ & $1.389(5)$ \\
\hline $\mathrm{C} 6-\mathrm{O} 3$ & $1.229(4)$ & $\mathrm{C} 4-\mathrm{C} 5$ & $1.482(5)$ \\
\hline $\mathrm{C} 6-\mathrm{C} 7$ & $1.484(4)$ & $\mathrm{C} 5-\mathrm{H} 5 \mathrm{a}$ & 0.96 \\
\hline $\mathrm{N} 3-\mathrm{O} 4$ & $1.223(4)$ & $\mathrm{C} 5-\mathrm{H} 5 \mathrm{~b}$ & 0.96 \\
\hline $\mathrm{N} 3-\mathrm{O} 5$ & $1.231(4)$ & $\mathrm{C} 5-\mathrm{H} 5 \mathrm{c}$ & 0.96 \\
\hline $\mathrm{N} 3-\mathrm{C} 9$ & $1.452(5)$ & $\mathrm{H} 5 \mathrm{a}-\mathrm{H} 5 \mathrm{~b}$ & 1.5677 \\
\hline $\mathrm{N} 4-\mathrm{O} 6$ & $1.220(4)$ & $\mathrm{H} 5 \mathrm{a}-\mathrm{H} 5 \mathrm{c}$ & 1.5677 \\
\hline $\mathrm{N} 4-\mathrm{O} 7$ & $1.213(4)$ & $\mathrm{H} 5 \mathrm{~b}-\mathrm{H} 5 \mathrm{c}$ & 1.5677 \\
\hline $\mathrm{N} 4-\mathrm{C} 11$ & $1.457(4)$ & $\mathrm{C} 12-\mathrm{C} 7$ & $1.433(4)$ \\
\hline $\mathrm{O} 1-\mathrm{C} 12$ & $1.277(4)$ & $\mathrm{C} 12-\mathrm{C} 11$ & $1.422(4)$ \\
\hline $\mathrm{O} 1-\mathrm{H} 2 \mathrm{a}$ & $1.22(5)$ & $\mathrm{C} 7-\mathrm{C} 8$ & $1.358(5)$ \\
\hline $\mathrm{O} 2-\mathrm{H} 2 \mathrm{a}$ & $1.34(5)$ & $\mathrm{C} 8-\mathrm{H} 8$ & 0.93 \\
\hline $\mathrm{C} 1-\mathrm{H} 1 \mathrm{a}$ & 0.96 & $\mathrm{C} 8-\mathrm{C} 9$ & $1.382(4)$ \\
\hline $\mathrm{C} 1-\mathrm{H} 1 \mathrm{~b}$ & 0.96 & $\mathrm{C} 9-\mathrm{C} 10$ & $1.384(4)$ \\
\hline $\mathrm{C} 1-\mathrm{H} 1 \mathrm{c}$ & 0.96 & $\mathrm{C} 10-\mathrm{H} 10$ & 0.93 \\
\hline $\mathrm{C} 1-\mathrm{C} 2$ & $1.494(5)$ & $\mathrm{C} 10-\mathrm{C} 11$ & $1.380(5)$ \\
\hline $\mathrm{N} 2-\mathrm{N} 1-\mathrm{C} 2$ & $108.2(3)$ & $\mathrm{N} 2-\mathrm{C} 4-\mathrm{C} 3$ & $106.8(3)$ \\
\hline $\mathrm{N} 2-\mathrm{N} 1-\mathrm{H} 1$ & $121(2)$ & $\mathrm{N} 2-\mathrm{C} 4-\mathrm{C} 5$ & 122.3 \\
\hline $\mathrm{C} 2-\mathrm{N} 1-\mathrm{H} 1$ & $131(2)$ & $\mathrm{C} 3-\mathrm{C} 4-\mathrm{C} 5$ & $130.9(3)$ \\
\hline $\mathrm{N} 1-\mathrm{N} 2-\mathrm{C} 4$ & $110.1(3)$ & $\mathrm{C} 4-\mathrm{C} 5-\mathrm{H} 5 \mathrm{a}$ & 109.47 \\
\hline $\mathrm{N} 1-\mathrm{N} 2-\mathrm{H} 2$ & $117(2)$ & $\mathrm{C} 4-\mathrm{C} 5-\mathrm{H} 5 \mathrm{~b}$ & 109.47 \\
\hline $\mathrm{C} 4-\mathrm{N} 2-\mathrm{H} 2$ & $132(2)$ & $\mathrm{C} 4-\mathrm{C} 5-\mathrm{H} 5 \mathrm{c}$ & 109.47 \\
\hline $\mathrm{O} 2-\mathrm{C} 6-\mathrm{O} 3$ & $121.3(3)$ & $\mathrm{H} 5 \mathrm{a}-\mathrm{C} 5-\mathrm{H} 5 \mathrm{~b}$ & 109.47 \\
\hline $\mathrm{O} 2-\mathrm{C} 6-\mathrm{C} 7$ & $117.2(3)$ & $\mathrm{H} 5 \mathrm{a}-\mathrm{C} 5-\mathrm{H} 5 \mathrm{c}$ & 109.47 \\
\hline $\mathrm{O} 3-\mathrm{C} 6-\mathrm{C} 7$ & $121.5(3)$ & $\mathrm{H} 5 \mathrm{~b}-\mathrm{C} 5-\mathrm{H} 5 \mathrm{c}$ & 109.47 \\
\hline $\mathrm{O} 4-\mathrm{N} 3-\mathrm{O} 5$ & $123.2(3)$ & $\mathrm{O} 1-\mathrm{C} 12-\mathrm{C} 7$ & 121.3 \\
\hline $\mathrm{O} 4-\mathrm{N} 3-\mathrm{C} 9$ & $118.1(3)$ & $\mathrm{O} 1-\mathrm{C} 12-\mathrm{C} 11$ & $124.1(3)$ \\
\hline $\mathrm{O} 5-\mathrm{N} 3-\mathrm{C} 9$ & $118.7(3)$ & $\mathrm{C} 7-\mathrm{C} 12-\mathrm{C} 11$ & 114.5 \\
\hline $\mathrm{O} 6-\mathrm{N} 4-\mathrm{O} 7$ & $122.1(3)$ & $\mathrm{C} 6-\mathrm{C} 7-\mathrm{C} 12$ & $119.5(3)$ \\
\hline $\mathrm{O} 6-\mathrm{N} 4-\mathrm{C} 11$ & $117.8(3)$ & $\mathrm{C} 6-\mathrm{C} 7-\mathrm{C} 8$ & $118.1(3)$ \\
\hline $\mathrm{O} 7-\mathrm{N} 4-\mathrm{C} 11$ & $120.1(3)$ & $\mathrm{C} 12-\mathrm{C} 7-\mathrm{C} 8$ & 122.3 \\
\hline $\mathrm{C} 12-\mathrm{O} 1-\mathrm{H} 2 \mathrm{a}$ & $106(2)$ & $\mathrm{C} 7-\mathrm{C} 8-\mathrm{H} 8$ & 119.87 \\
\hline $\mathrm{C} 6-\mathrm{O} 2-\mathrm{H} 2 \mathrm{a}$ & $106(2)$ & $\mathrm{C} 7-\mathrm{C} 8-\mathrm{C} 9$ & 120.3 \\
\hline $\mathrm{H} 1 \mathrm{a}-\mathrm{C} 1-\mathrm{H} 1 \mathrm{~b}$ & 109.47 & $\mathrm{H} 8-\mathrm{C} 8-\mathrm{C} 9$ & 119.87 \\
\hline $\mathrm{H} 1 \mathrm{a}-\mathrm{C} 1-\mathrm{H} 1 \mathrm{c}$ & 109.47 & $\mathrm{~N} 3-\mathrm{C} 9-\mathrm{C} 8$ & $119.6(3)$ \\
\hline $\mathrm{H} 1 \mathrm{a}-\mathrm{C} 1-\mathrm{C} 2$ & 109.47 & $\mathrm{~N} 3-\mathrm{C} 9-\mathrm{C} 10$ & $119.3(3)$ \\
\hline $\mathrm{H} 1 \mathrm{~b}-\mathrm{C} 1-\mathrm{H} 1 \mathrm{c}$ & 109.47 & $\mathrm{C} 8-\mathrm{C} 9-\mathrm{C} 10$ & $121.0(3)$ \\
\hline
\end{tabular}




$\begin{array}{llll}\mathrm{H} 1 \mathrm{~b}-\mathrm{C} 1-\mathrm{C} 2 & 109.47 & \mathrm{C} 9-\mathrm{C} 10-\mathrm{H} 10 & 120.77 \\ \mathrm{H} 1 \mathrm{c}-\mathrm{C} 1-\mathrm{C} 2 & 109.47 & \mathrm{C} 9-\mathrm{C} 10-\mathrm{C} 11 & 118.5(3) \\ \mathrm{N} 1-\mathrm{C} 2-\mathrm{C} 1 & 122.8(3) & \mathrm{H} 10-\mathrm{C} 10-\mathrm{C} 11 & 120.77 \\ \mathrm{~N} 1-\mathrm{C} 2-\mathrm{C} 3 & 108.1(3) & \mathrm{N} 4-\mathrm{C} 11-\mathrm{C} 12 & 120.9(3) \\ \mathrm{C} 1-\mathrm{C} 2-\mathrm{C} 3 & 129.1(3) & \mathrm{N} 4-\mathrm{C} 11-\mathrm{C} 10 & 115.7(3) \\ \mathrm{C} 2-\mathrm{C} 3-\mathrm{H} 3 & 126.58 & \mathrm{C} 12-\mathrm{C} 11-\mathrm{C} 10 & 123.3(3) \\ \mathrm{C} 2-\mathrm{C} 3-\mathrm{C} 4 & 106.8(3) & \mathrm{O} 1-\mathrm{H} 2 \mathrm{a}-\mathrm{O} 2 & 149(5) \\ \mathrm{H} 3-\mathrm{C} 3-\mathrm{C} 4 & 126.58 & & \end{array}$

Hydrogen-bond geometry $\left(\AA,{ }^{\circ}\right)$

\begin{tabular}{lllll}
\hline$D-\mathrm{H} \cdots A$ & $D-\mathrm{H}$ & $\mathrm{H} \cdots A$ & $D \cdots A$ & $D-\mathrm{H} \cdots A$ \\
\hline $\mathrm{C} 1-\mathrm{H} 1 a \cdots \mathrm{O} 7^{\mathrm{i}}$ & 0.96 & 2.49 & $3.176(5)$ & 128 \\
$\mathrm{C} 5-\mathrm{H} 5 a \cdots 4^{\mathrm{ii}}$ & 0.96 & 2.47 & $3.395(5)$ & 162 \\
$\mathrm{C} 10-\mathrm{H} 10 \cdots \mathrm{O} 6^{\mathrm{iii}}$ & 0.93 & 2.47 & $3.369(4)$ & 164 \\
$\mathrm{O} 1-\mathrm{H} 2 a \cdots \mathrm{O} 2$ & $1.22(5)$ & $1.34(5)$ & $2.476(3)$ & $149(5)$ \\
$\mathrm{O} 2-\mathrm{H} 2 a \cdots \mathrm{O} 1$ & $1.34(5)$ & $1.22(5)$ & $2.476(3)$ & $149(5)$ \\
$\mathrm{N} 1-\mathrm{H} 1 \cdots \mathrm{O} 1^{\mathrm{i}}$ & $1.11(5)$ & $1.92(5)$ & $2.799(4)$ & $133(3)$ \\
$\mathrm{N} 1-\mathrm{H} 1 \cdots \mathrm{O} 7^{\mathrm{i}}$ & $1.11(5)$ & $1.94(5)$ & $2.850(4)$ & $137(3)$ \\
$\mathrm{N} 2-\mathrm{H} 2 \cdots \mathrm{O} 3$ & $0.96(3)$ & $1.77(3)$ & $2.685(4)$ & $158(3)$
\end{tabular}

Symmetry codes: (i) $-x+1, y+1 / 2,-z+1$; (ii) $-x, y-3 / 2,-z+1$; (iii) $-x+1, y+1 / 2,-z+2$.

3-(1H-Imidazol-1-yl)propanaminium 2-carboxy-4,6-dinitrophenolate (TIYZIM)

Crystal data

$\mathrm{C}_{6} \mathrm{H}_{12} \mathrm{~N}_{3}{ }^{+} \cdot \mathrm{C}_{7} \mathrm{H}_{3} \mathrm{~N}_{2} \mathrm{O}_{7}^{-}$

$M_{r}=353.30$

Triclinic, $P \overline{1}$

$a=7.0109(4) \AA$

$b=10.6617(8) \AA$

$c=10.7454(7) \AA$

$\alpha=93.075(6)^{\circ}$

$\beta=95.863(5)^{\circ}$

$\gamma=104.944(6)^{\circ}$

$V=769.30(9) \AA^{3}$

Data collection

Agilent Xcalibur (Eos, Gemini) diffractometer

Graphite monochromator

Detector resolution: 16.0416 pixels $\mathrm{mm}^{-1}$

$\omega$ scans

Absorption correction: multi-scan

(CrysAlis PRO and CrysAlis RED; Agilent, 2012)

$T_{\min }=0.925, T_{\max }=1.000$

\section{Refinement}

Refinement on $F^{2}$

Least-squares matrix: full

$R[F>3 \sigma(F)]=0.041$
$Z=2$

$F(000)=368$

$D_{\mathrm{x}}=1.525 \mathrm{Mg} \mathrm{m}^{-3}$

$\mathrm{Cu} K \alpha$ radiation, $\lambda=1.54184 \AA$

Cell parameters from 2218 reflections

$\theta=4.2-72.3^{\circ}$

$\mu=1.09 \mathrm{~mm}^{-1}$

$T=173 \mathrm{~K}$

Irregular, yellow

$0.22 \times 0.14 \times 0.12 \mathrm{~mm}$

4664 measured reflections

2953 independent reflections

2426 reflections with $I>3 \sigma(I)$

$R_{\text {int }}=0.026$

$\theta_{\max }=72.5^{\circ}, \theta_{\min }=4.2^{\circ}$

$h=-8 \rightarrow 5$

$k=-12 \rightarrow 13$

$l=-13 \rightarrow 13$

$w R(F)=0.100$

$S=1.64$

2953 reflections 
229 parameters

0 restraints

46 constraints

Primary atom site location: structure-invariant direct methods

Hydrogen site location: difference Fourier map

$\mathrm{H}$ atoms treated by a mixture of independent and constrained refinement
Weighting scheme based on measured s.u.'s $w=$ $1 /\left(\sigma^{2}(I)+0.0004 I^{2}\right)$

$(\Delta / \sigma)_{\max }=0.013$

$\Delta \rho_{\max }=0.21 \mathrm{e} \AA^{-3}$

$\Delta \rho_{\min }=-0.18$ e $\AA^{-3}$

Extinction correction: B-C type 1 Lorentzian isotropic (Becker \& Coppens, 1974)

Extinction coefficient: 740 (130)

\section{Special details}

Geometry. All esds (except the esd in the dihedral angle between two 1.s. planes) are estimated using the full covariance matrix. The cell esds are taken into account individually in the estimation of esds in distances, angles and torsion angles; correlations between esds in cell parameters are only used when they are defined by crystal symmetry. An approximate (isotropic) treatment of cell esds is used for estimating esds involving l.s. planes.

Refinement. Number of fixed parameters: 12

Fractional atomic coordinates and isotropic or equivalent isotropic displacement parameters $\left(\AA^{2}\right)$

\begin{tabular}{|c|c|c|c|c|}
\hline & $x$ & $y$ & $z$ & $U_{\text {iso }} * / U_{\text {eq }}$ \\
\hline $\mathrm{O} 1 \mathrm{~b}$ & $-0.19161(17)$ & $0.67539(12)$ & $0.52684(11)$ & $0.0294(4)$ \\
\hline $\mathrm{O} 2 \mathrm{~b}$ & $-0.38292(17)$ & $0.47166(12)$ & $0.40838(12)$ & $0.0313(4)$ \\
\hline $\mathrm{O} 3 \mathrm{~b}$ & $-0.25511(17)$ & $0.37712(12)$ & $0.26158(12)$ & $0.0312(4)$ \\
\hline $\mathrm{O} 4 \mathrm{~b}$ & $0.41258(19)$ & $0.58639(13)$ & $0.16853(13)$ & $0.0365(5)$ \\
\hline $\mathrm{O} 5 \mathrm{~b}$ & $0.59776(18)$ & $0.75622(13)$ & $0.28239(14)$ & $0.0382(5)$ \\
\hline $\mathrm{O} 6 \mathrm{~b}$ & $0.3446(2)$ & $0.93719(14)$ & $0.62658(15)$ & $0.0474(5)$ \\
\hline $\mathrm{O} 7 \mathrm{~b}$ & 0.02822 (19) & $0.91655(13)$ & 0.61477 (13) & $0.0410(5)$ \\
\hline N1b & $0.1720(2)$ & $0.88469(14)$ & $0.58118(14)$ & $0.0301(5)$ \\
\hline $\mathrm{N} 2 \mathrm{~b}$ & $0.4396(2)$ & $0.67347(14)$ & $0.25363(14)$ & $0.0281(5)$ \\
\hline $\mathrm{C} 1 \mathrm{~b}$ & $-0.0455(2)$ & $0.68023(16)$ & $0.46268(15)$ & $0.0225(5)$ \\
\hline $\mathrm{C} 2 \mathrm{~b}$ & $-0.0572(2)$ & $0.57873(15)$ & $0.36597(15)$ & $0.0217(5)$ \\
\hline $\mathrm{C} 3 \mathrm{~b}$ & $0.0987(2)$ & $0.57933(16)$ & $0.29810(15)$ & $0.0228(5)$ \\
\hline $\mathrm{H} 3 \mathrm{~b}$ & 0.086172 & 0.512562 & 0.233219 & $0.0273 *$ \\
\hline $\mathrm{C} 4 \mathrm{~b}$ & $0.2738(2)$ & $0.67709(16)$ & $0.32422(15)$ & $0.0238(5)$ \\
\hline $\mathrm{C} 5 \mathrm{~b}$ & $0.2967(2)$ & $0.77664(16)$ & $0.41630(15)$ & $0.0244(5)$ \\
\hline $\mathrm{H} 5 \mathrm{~b}$ & 0.418494 & 0.842618 & 0.433677 & $0.0292^{*}$ \\
\hline C6b & $0.1392(2)$ & $0.77850(16)$ & $0.48267(15)$ & $0.0244(5)$ \\
\hline $\mathrm{C} 7 \mathrm{~b}$ & $-0.2405(2)$ & $0.46767(16)$ & $0.33986(15)$ & $0.0245(5)$ \\
\hline N1a & $-0.2235(2)$ & $0.05127(14)$ & $-0.17301(14)$ & $0.0305(5)$ \\
\hline $\mathrm{N} 2 \mathrm{a}$ & $-0.0146(2)$ & $0.22579(13)$ & $-0.06967(13)$ & $0.0239(4)$ \\
\hline $\mathrm{N} 3 \mathrm{a}$ & $0.3467(2)$ & $0.20531(13)$ & $0.28160(13)$ & $0.0254(4)$ \\
\hline C1a & $-0.0395(2)$ & $0.12591(16)$ & $-0.15749(16)$ & $0.0272(6)$ \\
\hline H1a & 0.06324 & 0.111094 & -0.202772 & $0.0327 *$ \\
\hline $\mathrm{C} 2 \mathrm{a}$ & -0.3211 & $0.10665(17)$ & $-0.08987(17)$ & $0.0314(6)$ \\
\hline $\mathrm{H} 2 \mathrm{a}$ & -0.457443 & 0.07432 & -0.079116 & $0.0377 *$ \\
\hline $\mathrm{C} 3 \mathrm{a}$ & $-0.1954(3)$ & $0.21356(17)$ & $-0.02572(17)$ & $0.0294(6)$ \\
\hline H3a & -0.225845 & 0.269084 & 0.03706 & $0.0353 *$ \\
\hline $\mathrm{C} 4 \mathrm{a}$ & $0.1719(2)$ & $0.32466(17)$ & $-0.02832(15)$ & $0.0268(5)$ \\
\hline H4aa & 0.242014 & 0.351332 & -0.101965 & $0.0322 *$ \\
\hline $\mathrm{H} 4 \mathrm{ab}$ & 0.142708 & 0.403798 & 0.008481 & $0.0322 *$ \\
\hline
\end{tabular}


supporting information

$\begin{array}{lllll}\text { C5a } & 0.3076(2) & 0.27759(16) & 0.06668(16) & 0.0271(5) \\ \text { H5aa } & 0.324487 & 0.193134 & 0.033664 & 0.0326^{*} \\ \text { H5ab } & 0.440667 & 0.340434 & 0.079114 & 0.0326^{*} \\ \text { C6a } & 0.2253(2) & 0.26206(16) & 0.19085(15) & 0.0279(6) \\ \text { H6aa } & 0.218227 & 0.347891 & 0.227027 & 0.0335^{*} \\ \text { H6ab } & 0.08712 & 0.206021 & 0.17726 & 0.0335^{*} \\ \text { H2b } & -0.339134 & 0.554144 & 0.46148 & 0.075(9)^{*} \\ \text { H3aa } & 0.329781 & 0.119532 & 0.260701 & 0.045(4)^{*} \\ \text { H3ab } & 0.475827 & 0.248704 & 0.284554 & 0.045(4)^{*} \\ \text { H3ac } & 0.313145 & 0.21209 & 0.359189 & 0.045(4)^{*}\end{array}$

Atomic displacement parameters $\left(\AA^{2}\right)$

\begin{tabular}{lllllll}
\hline & $U^{11}$ & $U^{22}$ & $U^{33}$ & $U^{12}$ & $U^{13}$ & $U^{23}$ \\
\hline O1b & $0.0268(6)$ & $0.0307(7)$ & $0.0277(6)$ & $0.0010(5)$ & $0.0098(5)$ & $-0.0050(5)$ \\
O2b & $0.0247(6)$ & $0.0313(7)$ & $0.0327(7)$ & $-0.0024(5)$ & $0.0088(5)$ & $-0.0060(5)$ \\
O3b & $0.0283(6)$ & $0.0285(7)$ & $0.0317(7)$ & $0.0002(5)$ & $0.0047(5)$ & $-0.0082(5)$ \\
O4b & $0.0371(7)$ & $0.0325(7)$ & $0.0413(8)$ & $0.0085(6)$ & $0.0167(6)$ & $-0.0042(6)$ \\
O5b & $0.0231(6)$ & $0.0380(8)$ & $0.0506(9)$ & $0.0010(6)$ & $0.0111(6)$ & $0.0014(6)$ \\
O6b & $0.0352(7)$ & $0.0408(8)$ & $0.0552(10)$ & $-0.0009(6)$ & $-0.0052(7)$ & $-0.0197(7)$ \\
O7b & $0.0398(7)$ & $0.0333(7)$ & $0.0470(8)$ & $0.0034(6)$ & $0.0162(6)$ & $-0.0121(6)$ \\
N1b & $0.0326(8)$ & $0.0241(8)$ & $0.0301(8)$ & $0.0012(6)$ & $0.0060(6)$ & $-0.0023(6)$ \\
N2b & $0.0260(7)$ & $0.0259(7)$ & $0.0343(8)$ & $0.0074(6)$ & $0.0092(6)$ & $0.0063(6)$ \\
C1b & $0.0234(8)$ & $0.0236(8)$ & $0.0201(8)$ & $0.0054(6)$ & $0.0034(6)$ & $0.0027(6)$ \\
C2b & $0.0218(8)$ & $0.0215(8)$ & $0.0207(8)$ & $0.0036(6)$ & $0.0019(6)$ & $0.0029(6)$ \\
C3b & $0.0265(8)$ & $0.0220(8)$ & $0.0208(8)$ & $0.0077(7)$ & $0.0043(6)$ & $0.0017(6)$ \\
C4b & $0.0220(8)$ & $0.0254(8)$ & $0.0260(8)$ & $0.0080(7)$ & $0.0064(7)$ & $0.0057(7)$ \\
C5b & $0.0220(8)$ & $0.0220(8)$ & $0.0269(8)$ & $0.0015(6)$ & $0.0023(7)$ & $0.0050(7)$ \\
C6b & $0.0276(8)$ & $0.0210(8)$ & $0.0230(8)$ & $0.0045(7)$ & $0.0018(7)$ & $-0.0001(6)$ \\
C7b & $0.0247(8)$ & $0.0261(8)$ & $0.0216(8)$ & $0.0051(7)$ & $0.0021(6)$ & $0.0007(6)$ \\
N1a & $0.0292(8)$ & $0.0252(8)$ & $0.0349(8)$ & $0.0050(6)$ & $0.0011(6)$ & $-0.0007(6)$ \\
N2a & $0.0247(7)$ & $0.0234(7)$ & $0.0229(7)$ & $0.0054(6)$ & $0.0034(5)$ & $0.0002(6)$ \\
N3a & $0.0268(7)$ & $0.0229(7)$ & $0.0245(7)$ & $0.0041(6)$ & $0.0021(6)$ & $-0.0026(6)$ \\
C1a & $0.0286(9)$ & $0.0260(9)$ & $0.0278(9)$ & $0.0087(7)$ & $0.0051(7)$ & $-0.0018(7)$ \\
C2a & $0.0269(9)$ & $0.0316(10)$ & $0.0352(10)$ & $0.0047(7)$ & $0.0073(7)$ & $0.0060(8)$ \\
C3a & $0.0301(9)$ & $0.0311(9)$ & $0.0289(9)$ & $0.0095(7)$ & $0.0092(7)$ & $0.0016(7)$ \\
C4a & $0.0277(8)$ & $0.0248(8)$ & $0.0248(8)$ & $0.0009(7)$ & $0.0052(7)$ & $0.0000(7)$ \\
C5a & $0.0237(8)$ & $0.0295(9)$ & $0.0260(9)$ & $0.0030(7)$ & $0.0055(7)$ & $-0.0021(7)$ \\
C6a & $0.0301(9)$ & $0.0306(9)$ & $0.0261(9)$ & $0.0125(7)$ & $0.0053(7)$ & $0.0016(7)$ \\
& & & & & &
\end{tabular}

Geometric parameters $\left(\AA,{ }^{\circ}\right)$

\begin{tabular}{llll}
\hline $\mathrm{O} 1 \mathrm{~b}-\mathrm{C} 1 \mathrm{~b}$ & $1.284(2)$ & $\mathrm{N} 1 \mathrm{a}-\mathrm{C} 2 \mathrm{a}$ & $1.376(3)$ \\
$\mathrm{O} 2 \mathrm{~b}-\mathrm{C} 7 \mathrm{~b}$ & $1.308(2)$ & $\mathrm{N} 2 \mathrm{a}-\mathrm{Cla}$ & $1.348(2)$ \\
$\mathrm{O} 2 \mathrm{~b}-\mathrm{H} 2 \mathrm{~b}$ & $0.9820(12)$ & $\mathrm{N} 2 \mathrm{a}-\mathrm{C} 3 \mathrm{a}$ & $1.375(2)$ \\
$\mathrm{O} 3 \mathrm{~b}-\mathrm{C} 7 \mathrm{~b}$ & $1.222(2)$ & $\mathrm{N} 2 \mathrm{a}-\mathrm{C} 4 \mathrm{a}$ & $1.4622(19)$ \\
$\mathrm{O} 4 \mathrm{~b}-\mathrm{N} 2 \mathrm{~b}$ & $1.232(2)$ & $\mathrm{N} 3 \mathrm{a}-\mathrm{C} 6 \mathrm{a}$ & $1.483(2)$ \\
$\mathrm{O} 5 \mathrm{~b}-\mathrm{N} 2 \mathrm{~b}$ & $1.2248(17)$ & $\mathrm{N} 3 \mathrm{a}-\mathrm{H} 3 \mathrm{aa}$ & $0.9042(14)$
\end{tabular}




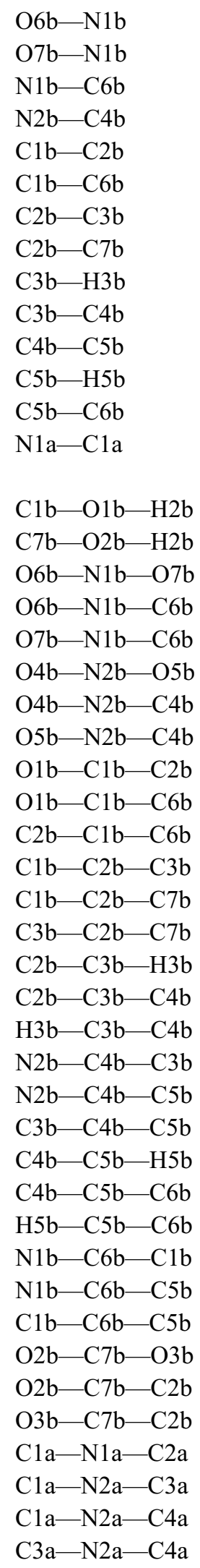

$1.2313(18)$

1.225 (2)

1.464 (2)

$1.458(2)$

$1.440(2)$

$1.428(2)$

1.373 (2)

$1.496(2)$

0.95

$1.382(2)$

$1.378(2)$

0.95

$1.378(2)$

$1.318(2)$

$99.82(10)$

$106.90(11)$

$123.30(15)$

$117.72(16)$

$118.97(13)$

$123.59(16)$

$117.89(12)$

118.52 (14)

$120.19(13)$

$124.76(15)$

$115.00(15)$

$121.54(13)$

$119.88(15)$

$118.56(14)$

120.01

119.99 (15)

120.01

119.03 (14)

$119.24(13)$

121.73 (16)

120.69

118.62 (13)

120.69

$120.26(15)$

$116.59(13)$

$123.09(15)$

$121.86(14)$

115.78 (14)

$122.33(16)$

$105.01(14)$

$106.69(13)$

$125.73(15)$

$127.56(14)$
N3a- H3ab

0.9009 (13)

$\mathrm{N} 3 \mathrm{a}-\mathrm{H} 3 \mathrm{ac}$

$\mathrm{C} 1 \mathrm{a}-\mathrm{H} 1 \mathrm{a}$

$\mathrm{C} 2 \mathrm{a}-\mathrm{H} 2 \mathrm{a}$

$\mathrm{C} 2 \mathrm{a}-\mathrm{C} 3 \mathrm{a}$

$\mathrm{C} 3 \mathrm{a}-\mathrm{H} 3 \mathrm{a}$

$\mathrm{C} 4 \mathrm{a}-\mathrm{H} 4 \mathrm{aa}$

$\mathrm{C} 4 \mathrm{a}-\mathrm{H} 4 \mathrm{ab}$

$\mathrm{C} 4 \mathrm{a}-\mathrm{C} 5 \mathrm{a}$

$\mathrm{C} 5 \mathrm{a}-\mathrm{H} 5 \mathrm{aa}$

$\mathrm{C} 5 \mathrm{a}-\mathrm{H} 5 \mathrm{ab}$

$\mathrm{C} 5 \mathrm{a}-\mathrm{C} 6 \mathrm{a}$

C6a-H6aa

C6a-H6ab

$\mathrm{C} 6 \mathrm{a}-\mathrm{N} 3 \mathrm{a}-\mathrm{H} 3 \mathrm{ab}$

$\mathrm{C} 6 \mathrm{a}-\mathrm{N} 3 \mathrm{a}-\mathrm{H} 3 \mathrm{ac}$

H3aa-N3a-H3ab

H3aa-N3a-H3ac

$\mathrm{H} 3 \mathrm{ab}-\mathrm{N} 3 \mathrm{a}-\mathrm{H} 3 \mathrm{ac}$

$\mathrm{N} 1 \mathrm{a}-\mathrm{C} 1 \mathrm{a}-\mathrm{N} 2 \mathrm{a}$

$\mathrm{N} 1 \mathrm{a}-\mathrm{Cla}-\mathrm{H} 1 \mathrm{a}$

$\mathrm{N} 2 \mathrm{a}-\mathrm{C} 1 \mathrm{a}-\mathrm{H} 1 \mathrm{a}$

$\mathrm{N} 1 \mathrm{a}-\mathrm{C} 2 \mathrm{a}-\mathrm{H} 2 \mathrm{a}$

$\mathrm{N} 1 \mathrm{a}-\mathrm{C} 2 \mathrm{a}-\mathrm{C} 3 \mathrm{a}$

$\mathrm{H} 2 \mathrm{a}-\mathrm{C} 2 \mathrm{a}-\mathrm{C} 3 \mathrm{a}$

$\mathrm{N} 2 \mathrm{a}-\mathrm{C} 3 \mathrm{a}-\mathrm{C} 2 \mathrm{a}$

$\mathrm{N} 2 \mathrm{a}-\mathrm{C} 3 \mathrm{a}-\mathrm{H} 3 \mathrm{a}$

$\mathrm{C} 2 \mathrm{a}-\mathrm{C} 3 \mathrm{a}-\mathrm{H} 3 \mathrm{a}$

$\mathrm{N} 2 \mathrm{a}-\mathrm{C} 4 \mathrm{a}-\mathrm{H} 4 \mathrm{aa}$

$\mathrm{N} 2 \mathrm{a}-\mathrm{C} 4 \mathrm{a}-\mathrm{H} 4 \mathrm{ab}$

$\mathrm{N} 2 \mathrm{a}-\mathrm{C} 4 \mathrm{a}-\mathrm{C} 5 \mathrm{a}$

$\mathrm{H} 4 \mathrm{aa}-\mathrm{C} 4 \mathrm{a}-\mathrm{H} 4 \mathrm{ab}$

$\mathrm{H} 4 \mathrm{aa}-\mathrm{C} 4 \mathrm{a}-\mathrm{C} 5 \mathrm{a}$

$\mathrm{H} 4 \mathrm{ab}-\mathrm{C} 4 \mathrm{a}-\mathrm{C} 5 \mathrm{a}$

$\mathrm{C} 4 \mathrm{a}-\mathrm{C} 5 \mathrm{a}-\mathrm{H} 5 \mathrm{aa}$

$\mathrm{C} 4 \mathrm{a}-\mathrm{C} 5 \mathrm{a}-\mathrm{H} 5 \mathrm{ab}$

$\mathrm{C} 4 \mathrm{a}-\mathrm{C} 5 \mathrm{a}-\mathrm{C} 6 \mathrm{a}$

H5aa-C $5 \mathrm{a}-\mathrm{H} 5 \mathrm{ab}$

H5aa-C5a-C6a

$\mathrm{H} 5 \mathrm{ab}-\mathrm{C} 5 \mathrm{a}-\mathrm{C} 6 \mathrm{a}$

N3a-C6a-C5a

N3a-C6a-H6aa

$\mathrm{N} 3 \mathrm{a}-\mathrm{C} 6 \mathrm{a}-\mathrm{H} 6 \mathrm{ab}$

C5a-C6a-H6aa

$\mathrm{C} 5 \mathrm{a}-\mathrm{C} 6 \mathrm{a}-\mathrm{H} 6 \mathrm{ab}$

H6aa-C6a-H6ab

$\mathrm{O} 1 \mathrm{~b}-\mathrm{H} 2 \mathrm{~b}-\mathrm{O} 2 \mathrm{~b}$
0.8932 (14)

0.95

0.95

1.351 (2)

0.95

0.99

0.99

1.517 (2)

0.99

0.99

1.507 (2)

0.99

0.99

$109.46(13)$

$111.76(15)$

$110.21(16)$

$106.71(14)$

$107.27(13)$

111.91 (16)

124.04

124.04

124.86

$110.28(15)$

124.86

106.10 (16)

126.95

126.95

109.47

109.47

112.62 (14)

106.12

109.47

109.47

109.47

109.47

$111.50(15)$

107.36

109.47

109.47

112.35 (15)

109.47

109.47

109.47

109.47

106.43

156.29 (9) 
Hydrogen-bond geometry $\left(\AA,{ }^{o}\right)$

\begin{tabular}{lllll}
\hline$D-\mathrm{H} \cdots A$ & $D-\mathrm{H}$ & $\mathrm{H} \cdots A$ & $D \cdots A$ & $D-\mathrm{H} \cdots A$ \\
\hline $\mathrm{C} 4 a-\mathrm{H} 4 a a \cdots \mathrm{O} 4 b^{\mathrm{i}}$ & 0.99 & 2.53 & $3.359(2)$ & 141 \\
$\mathrm{O} 2 b-\mathrm{H} 2 b \cdots \mathrm{O} 1 b$ & $0.9820(12)$ & $1.5161(11)$ & $2.4473(16)$ & $156.29(9)$ \\
$\mathrm{O} 2 b-\mathrm{H} 2 b \cdots \mathrm{C} 1 b$ & $0.9820(12)$ & $2.1471(15)$ & $2.7833(18)$ & $121.00(8)$ \\
$\mathrm{N} 3 a-\mathrm{H} 3 a a \cdots \mathrm{N} 1 a^{\mathrm{ii}}$ & $0.9042(14)$ & $1.9318(14)$ & $2.797(2)$ & $159.6(1)$ \\
$\mathrm{N} 3 a-\mathrm{H} 3 a b \cdots \mathrm{O} 2 b^{\mathrm{iii}}$ & $0.9009(13)$ & $2.5650(12)$ & $3.1297(17)$ & $121.35(10)$ \\
$\mathrm{N} 3 a-\mathrm{H} 3 a b \cdots \mathrm{O} 3 b^{\mathrm{iii}}$ & $0.9009(13)$ & $2.0721(11)$ & $2.9537(17)$ & $165.79(10)$ \\
$\mathrm{N} 3 a-\mathrm{H} 3 a c \cdots \mathrm{O} 1 b^{\mathrm{iv}}$ & $0.8932(14)$ & $2.0610(13)$ & $2.815(2)$ & $141.47(11)$ \\
$\mathrm{N} 3 a-\mathrm{H} 3 a c \cdots \mathrm{O} 7 b^{\mathrm{iv}}$ & $0.8932(14)$ & $2.4844(13)$ & $2.9712(19)$ & $114.74(8)$ \\
\hline
\end{tabular}

Symmetry codes: (i) $-x+1,-y+1,-z$; (ii) $-x,-y,-z$; (iii) $x+1, y, z$; (iv) $-x,-y+1,-z+1$.

4-\{[(5-Methylisoxazol-3-yl)amino]sulfonyl\}anilinium 2-hydroxy-3,5-dinitrobenzoate (TUJPEV)

Crystal data

$\mathrm{C}_{10} \mathrm{H}_{12} \mathrm{~N}_{3} \mathrm{O}_{3} \mathrm{~S}^{+} \cdot \mathrm{C}_{7} \mathrm{H}_{3} \mathrm{~N}_{2} \mathrm{O}_{7}^{-}$

$M_{r}=481.41$

Triclinic, $P \overline{1}$

Hall symbol: -P 1

$a=8.5551(1) \AA$

$b=10.5000(2) \AA$

$c=12.7576(3) \AA$

$\alpha=106.463(1)^{\circ}$

$\beta=100.913(1)^{\circ}$

$\gamma=108.272(1)^{\circ}$

$V=993.72(3) \AA^{3}$

\section{Data collection}

\section{Bruker Kappa APEXII CCD} diffractometer

Radiation source: fine-focus sealed tube

Graphite monochromator

$\omega$ and $\varphi$ scan

Absorption correction: multi-scan

(SADABS; Bruker, 2004)

$T_{\min }=0.955, T_{\max }=0.964$

\section{Refinement}

Refinement on $F^{2}$

Least-squares matrix: full

$R[F>3 \sigma(F)]=0.044$

$w R(F)=0.104$

$S=1.95$

6717 reflections

301 parameters

0 restraints

48 constraints

Primary atom site location: structure-invariant direct methods
$Z=2$

$F(000)=496$

$D_{\mathrm{x}}=1.609 \mathrm{Mg} \mathrm{m}^{-3}$

Mo $K \alpha$ radiation, $\lambda=0.71073 \AA$

Cell parameters from 6718 reflections

$\theta=1.8-32.6^{\circ}$

$\mu=0.23 \mathrm{~mm}^{-1}$

$T=296 \mathrm{~K}$

Prism, yellow

$0.20 \times 0.20 \times 0.16 \mathrm{~mm}$

24261 measured reflections

6717 independent reflections

4398 reflections with $I>3 \sigma(I)$

$R_{\text {int }}=0.030$

$\theta_{\max }=32.6^{\circ}, \theta_{\min }=1.8^{\circ}$

$h=-12 \rightarrow 12$

$k=-15 \rightarrow 15$

$l=-19 \rightarrow 16$

Secondary atom site location: difference Fourier map

Hydrogen site location: difference Fourier map

$\mathrm{H}$ atoms treated by a mixture of independent and constrained refinement

Weighting scheme based on measured s.u.'s $w=$ $1 /\left(\sigma^{2}(I)+0.0004 I^{2}\right)$

$(\Delta / \sigma)_{\max }=0.013$

$\Delta \rho_{\max }=0.31$ e $\AA^{-3}$

$\Delta \rho_{\min }=-0.35$ e $\AA^{-3}$ 


\section{Special details}

Geometry. Bond distances, angles etc. have been calculated using the rounded fractional coordinates. All su's are estimated from the variances of the (full) variance-covariance matrix. The cell e.s.d.'s are taken into account in the estimation of distances, angles and torsion angles

Refinement. Refinement on $F^{2}$ for ALL reflections except those flagged by the user for potential systematic errors. Weighted $R$-factors $w R$ and all goodnesses of fit $S$ are based on $F^{2}$, conventional $R$-factors $R$ are based on $F$, with $F$ set to zero for negative $F^{2}$. The observed criterion of $F^{2}>\sigma\left(F^{2}\right)$ is used only for calculating - $R$-factor-obs etc. and is not relevant to the choice of reflections for refinement. $R$-factors based on $F^{2}$ are statistically about twice as large as those based on $F$, and $R$-factors based on ALL data will be even larger.

Number of fixed parameters: 9

Fractional atomic coordinates and isotropic or equivalent isotropic displacement parameters $\left(\AA^{2}\right)$

\begin{tabular}{|c|c|c|c|c|}
\hline & $x$ & $y$ & $z$ & $U_{\text {iso }} * / U_{\text {eq }}$ \\
\hline S1 & $-0.02012(4)$ & $0.65156(4)$ & $0.38514(3)$ & $0.03475(15)$ \\
\hline $\mathrm{O} 1$ & $-0.12136(12)$ & $0.56298(10)$ & $0.26952(9)$ & $0.0472(5)$ \\
\hline $\mathrm{O} 2$ & $-0.09555(13)$ & $0.65440(11)$ & $0.47594(9)$ & $0.0468(5)$ \\
\hline $\mathrm{O} 3$ & $0.47434(14)$ & $0.55510(13)$ & $0.32058(11)$ & $0.0626(6)$ \\
\hline N1 & $0.29155(15)$ & $1.24477(12)$ & $0.38430(11)$ & $0.0434(6)$ \\
\hline N2 & $0.13845(14)$ & $0.60248(12)$ & $0.42019(10)$ & $0.0376(5)$ \\
\hline N3 & $0.39246(16)$ & $0.57705(15)$ & $0.40555(12)$ & $0.0536(6)$ \\
\hline $\mathrm{C} 1$ & $0.07464(15)$ & $0.82919(13)$ & $0.38935(12)$ & $0.0322(5)$ \\
\hline $\mathrm{C} 2$ & $0.21693(19)$ & $0.93183(16)$ & $0.47844(13)$ & $0.0511(7)$ \\
\hline $\mathrm{C} 3$ & $0.28732(19)$ & $1.06857(16)$ & $0.47796(14)$ & $0.0514(7)$ \\
\hline $\mathrm{C} 4$ & $0.21467(16)$ & $1.10142(13)$ & $0.38945(12)$ & $0.0344(6)$ \\
\hline $\mathrm{C} 5$ & $0.07052(18)$ & $1.00124(15)$ & $0.30229(13)$ & $0.0444(7)$ \\
\hline C6 & $0.00046(17)$ & $0.86380(15)$ & $0.30181(13)$ & $0.0421(6)$ \\
\hline $\mathrm{C} 7$ & $0.24788(17)$ & $0.58299(13)$ & $0.35471(13)$ & $0.0355(6)$ \\
\hline $\mathrm{C} 8$ & $0.2306(2)$ & $0.56568(16)$ & $0.23936(14)$ & $0.0473(7)$ \\
\hline C9 & $0.3761(2)$ & $0.55015(16)$ & $0.22381(16)$ & $0.0511(8)$ \\
\hline $\mathrm{C} 10$ & $0.4452(3)$ & $0.5287(2)$ & $0.12416(18)$ & $0.0766(11)$ \\
\hline $\mathrm{O} 4$ & $0.51685(14)$ & $0.76882(14)$ & $0.75724(10)$ & $0.0657(6)$ \\
\hline O5 & $0.25004(13)$ & $0.72390(11)$ & $0.65907(9)$ & $0.0495(5)$ \\
\hline O6 & $0.03442(11)$ & $0.74382(11)$ & $0.75483(8)$ & $0.0431(4)$ \\
\hline O7 & $-0.13539(17)$ & $0.8490(2)$ & $0.89544(14)$ & $0.0980(10)$ \\
\hline $\mathrm{O} 8$ & $-0.12065(16)$ & $0.7660(2)$ & $1.03000(12)$ & $0.0975(9)$ \\
\hline O9 & $0.46550(16)$ & $0.90412(15)$ & $1.25865(10)$ & $0.0717(7)$ \\
\hline $\mathrm{O} 10$ & $0.66293(15)$ & $0.90569(15)$ & $1.17611(11)$ & $0.0749(7)$ \\
\hline N4 & $-0.06158(16)$ & $0.80710(17)$ & $0.96062(13)$ & $0.0598(7)$ \\
\hline N5 & $0.51466(16)$ & $0.88865(13)$ & $1.17407(12)$ & $0.0488(6)$ \\
\hline C11 & $0.31789(15)$ & $0.78551(13)$ & $0.85956(12)$ & $0.0326(5)$ \\
\hline $\mathrm{C} 12$ & $0.14714(16)$ & $0.77857(13)$ & $0.85292(12)$ & $0.0328(5)$ \\
\hline C13 & $0.10840(16)$ & $0.80786(15)$ & 0.95755 (13) & $0.0389(6)$ \\
\hline C14 & $0.22578(17)$ & $0.84024(15)$ & $1.06095(13)$ & $0.0403(6)$ \\
\hline $\mathrm{C} 15$ & $0.38931(16)$ & $0.84763(14)$ & $1.06263(12)$ & $0.0365(6)$ \\
\hline $\mathrm{C} 16$ & $0.43735(16)$ & $0.82163(13)$ & $0.96389(12)$ & $0.0356(6)$ \\
\hline $\mathrm{C} 17$ & $0.36856(18)$ & $0.75728(15)$ & $0.75210(13)$ & $0.0400(6)$ \\
\hline H1a & 0.188848 & 1.256572 & 0.342213 & $0.088(4)^{*}$ \\
\hline
\end{tabular}




$\begin{array}{lllll}\text { H1b } & 0.353568 & 1.297412 & 0.446823 & 0.088(4)^{*} \\ \text { H1c } & 0.348288 & 1.238627 & 0.338232 & 0.088(4)^{*} \\ \text { H2 } & 0.265121 & 0.908789 & 0.538567 & 0.0613^{*} \\ \text { H2a } & 0.186499 & 0.630039 & 0.50234 & 0.068(5)^{*} \\ \text { H3 } & 0.383907 & 1.138439 & 0.537561 & 0.0617^{*} \\ \text { H5 } & 0.020222 & 1.025766 & 0.243815 & 0.0533^{*} \\ \text { H6 } & -0.096798 & 0.794538 & 0.242417 & 0.0506^{*} \\ \text { H8 } & 0.139615 & 0.565054 & 0.185878 & 0.0568^{*} \\ \text { H10a } & 0.551947 & 0.608597 & 0.142286 & 0.1149^{*} \\ \text { H10b } & 0.362955 & 0.522551 & 0.058321 & 0.1149^{*} \\ \text { H10c } & 0.46505 & 0.441064 & 0.1077 & 0.1149^{*} \\ \text { H14 } & 0.194965 & 0.856742 & 1.128171 & 0.0484^{*} \\ \text { H16 } & 0.549353 & 0.82833 & 0.967386 & 0.0428^{*} \\ \text { H6a } & 0.115577 & 0.727699 & 0.69023 & 0.132(9)^{*}\end{array}$

Atomic displacement parameters $\left(\AA^{2}\right)$

\begin{tabular}{|c|c|c|c|c|c|c|}
\hline & $U^{11}$ & $U^{22}$ & $U^{33}$ & $U^{12}$ & $U^{13}$ & $U^{23}$ \\
\hline S1 & $0.03683(17)$ & $0.03321(18)$ & $0.0360(2)$ & $0.01370(13)$ & $0.01260(14)$ & $0.01423(16)$ \\
\hline $\mathrm{O} 1$ & $0.0462(5)$ & $0.0373(5)$ & $0.0422(7)$ & $0.0089(4)$ & $0.0009(5)$ & $0.0087(5)$ \\
\hline $\mathrm{O} 2$ & $0.0524(6)$ & $0.0510(6)$ & $0.0532(7)$ & $0.0246(5)$ & $0.0309(5)$ & $0.0276(6)$ \\
\hline $\mathrm{O} 3$ & $0.0518(6)$ & $0.0718(8)$ & $0.0660(9)$ & $0.0313(6)$ & $0.0248(6)$ & $0.0153(7)$ \\
\hline N1 & $0.0510(7)$ & $0.0374(6)$ & $0.0502(9)$ & $0.0178(5)$ & $0.0268(6)$ & $0.0202(6)$ \\
\hline N2 & $0.0476(6)$ & $0.0413(6)$ & $0.0327(7)$ & $0.0235(5)$ & $0.0154(5)$ & $0.0169(6)$ \\
\hline N3 & $0.0495(7)$ & $0.0628(9)$ & $0.0487(9)$ & $0.0283(6)$ & $0.0154(6)$ & $0.0136(7)$ \\
\hline $\mathrm{C} 1$ & $0.0350(6)$ & $0.0317(7)$ & $0.0316(8)$ & $0.0143(5)$ & $0.0112(5)$ & $0.0120(6)$ \\
\hline $\mathrm{C} 2$ & $0.0588(9)$ & $0.0433(9)$ & $0.0382(9)$ & $0.0107(7)$ & $-0.0053(7)$ & $0.0197(8)$ \\
\hline $\mathrm{C} 3$ & $0.0520(9)$ & $0.0380(8)$ & $0.0432(10)$ & $0.0029(7)$ & $-0.0033(7)$ & $0.0129(8)$ \\
\hline $\mathrm{C} 4$ & $0.0400(7)$ & $0.0320(7)$ & $0.0393(9)$ & $0.0172(6)$ & $0.0208(6)$ & $0.0153(6)$ \\
\hline $\mathrm{C} 5$ & $0.0478(8)$ & $0.0446(8)$ & $0.0438(10)$ & $0.0195(7)$ & $0.0064(7)$ & $0.0235(8)$ \\
\hline C6 & $0.0392(7)$ & $0.0397(8)$ & $0.0403(9)$ & $0.0118(6)$ & $0.0004(6)$ & $0.0159(7)$ \\
\hline $\mathrm{C} 7$ & $0.0414(7)$ & $0.0275(7)$ & $0.0374(9)$ & $0.0132(5)$ & $0.0131(6)$ & $0.0113(6)$ \\
\hline $\mathrm{C} 8$ & $0.0553(9)$ & $0.0506(9)$ & $0.0426(10)$ & $0.0238(7)$ & $0.0203(7)$ & $0.0194(8)$ \\
\hline C9 & $0.0605(9)$ & $0.0379(8)$ & $0.0574(12)$ & $0.0169(7)$ & $0.0325(9)$ & $0.0141(8)$ \\
\hline C10 & $0.0921(14)$ & $0.0731(13)$ & $0.0819(15)$ & $0.0351(12)$ & $0.0607(13)$ & $0.0275(13)$ \\
\hline $\mathrm{O} 4$ & $0.0483(6)$ & $0.0981(10)$ & $0.0513(8)$ & $0.0274(6)$ & $0.0254(5)$ & $0.0227(7)$ \\
\hline $\mathrm{O} 5$ & $0.0578(6)$ & $0.0613(7)$ & $0.0295(6)$ & $0.0241(5)$ & $0.0132(5)$ & $0.0156(5)$ \\
\hline O6 & $0.0404(5)$ & $0.0525(6)$ & $0.0322(6)$ & $0.0196(4)$ & $0.0028(4)$ & $0.0136(5)$ \\
\hline $\mathrm{O} 7$ & $0.0700(8)$ & $0.1711(16)$ & $0.0831(11)$ & $0.0810(10)$ & $0.0212(8)$ & $0.0529(11)$ \\
\hline $\mathrm{O} 8$ & $0.0537(7)$ & $0.1721(16)$ & $0.0566(9)$ & $0.0299(9)$ & $0.0287(7)$ & $0.0359(10)$ \\
\hline O9 & $0.0770(8)$ & $0.0950(10)$ & $0.0308(7)$ & $0.0267(7)$ & $0.0041(6)$ & $0.0211(7)$ \\
\hline $\mathrm{O} 10$ & $0.0480(7)$ & $0.0991(10)$ & $0.0639(9)$ & $0.0302(6)$ & $-0.0064(6)$ & $0.0241(8)$ \\
\hline N4 & $0.0396(7)$ & $0.0856(11)$ & $0.0419(9)$ & $0.0249(7)$ & $0.0082(6)$ & $0.0084(8)$ \\
\hline N5 & $0.0478(7)$ & $0.0458(7)$ & $0.0405(9)$ & $0.0147(6)$ & $-0.0044(6)$ & $0.0138(7)$ \\
\hline C11 & $0.0346(6)$ & $0.0281(6)$ & $0.0318(8)$ & $0.0107(5)$ & $0.0078(5)$ & $0.0096(6)$ \\
\hline C12 & $0.0351(6)$ & $0.0301(7)$ & $0.0291(8)$ & $0.0112(5)$ & $0.0039(5)$ & $0.0104(6)$ \\
\hline C13 & $0.0330(6)$ & $0.0444(8)$ & $0.0356(9)$ & $0.0154(6)$ & $0.0079(6)$ & $0.0108(7)$ \\
\hline C14 & $0.0424(7)$ & $0.0454(8)$ & $0.0301(8)$ & $0.0162(6)$ & $0.0100(6)$ & $0.0112(7)$ \\
\hline
\end{tabular}




$\begin{array}{lllllll}\mathrm{C} 15 & 0.0370(7) & 0.0341(7) & 0.0297(8) & 0.0107(5) & -0.0009(6) & 0.0104(6) \\ \mathrm{C} 16 & 0.0321(6) & 0.0324(7) & 0.0393(9) & 0.0124(5) & 0.0061(6) & 0.0120(6) \\ \mathrm{C} 17 & 0.0411(7) & 0.0393(8) & 0.0385(9) & 0.0139(6) & 0.0130(6) & 0.0143(7)\end{array}$

Geometric parameters $\left(\stackrel{A}{\circ}{ }^{\circ}\right)$

\begin{tabular}{|c|c|c|c|}
\hline $\mathrm{S} 1-\mathrm{O} 1$ & $1.4228(9)$ & $\mathrm{C} 9-\mathrm{C} 10$ & $1.491(3)$ \\
\hline $\mathrm{S} 1-\mathrm{O} 2$ & $1.4264(13)$ & $\mathrm{C} 10-\mathrm{H} 10 \mathrm{a}$ & 0.96 \\
\hline $\mathrm{S} 1-\mathrm{N} 2$ & $1.6264(14)$ & $\mathrm{C} 10-\mathrm{H} 10 \mathrm{~b}$ & 0.96 \\
\hline $\mathrm{O} 3-\mathrm{N} 3$ & $1.402(2)$ & $\mathrm{C} 10-\mathrm{H} 10 \mathrm{c}$ & 0.96 \\
\hline $\mathrm{O} 3-\mathrm{C} 9$ & $1.333(2)$ & $\mathrm{O} 4-\mathrm{C} 17$ & $1.223(2)$ \\
\hline N1-C4 & $1.468(2)$ & $\mathrm{O} 5-\mathrm{C} 17$ & $1.2827(18)$ \\
\hline $\mathrm{N} 1-\mathrm{H} 1 \mathrm{a}$ & $1.0015(14)$ & O5-H6a & $1.2945(12)$ \\
\hline $\mathrm{N} 1-\mathrm{H} 1 \mathrm{~b}$ & $0.7932(11)$ & $\mathrm{O} 6-\mathrm{C} 12$ & $1.3010(16)$ \\
\hline $\mathrm{N} 1-\mathrm{H} 1 \mathrm{c}$ & $0.8315(15)$ & O6-H6a & $1.1837(11)$ \\
\hline $\mathrm{N} 2-\mathrm{C} 7$ & $1.391(2)$ & $\mathrm{O} 7-\mathrm{N} 4$ & $1.211(3)$ \\
\hline $\mathrm{N} 2-\mathrm{H} 2 \mathrm{a}$ & $0.9703(12)$ & $\mathrm{O} 8-\mathrm{N} 4$ & $1.214(2)$ \\
\hline $\mathrm{N} 3-\mathrm{C} 7$ & $1.312(2)$ & $\mathrm{O} 9-\mathrm{N} 5$ & $1.218(2)$ \\
\hline $\mathrm{C} 1-\mathrm{C} 2$ & $1.3764(15)$ & $\mathrm{O} 10-\mathrm{N} 5$ & $1.218(2)$ \\
\hline $\mathrm{C} 1-\mathrm{C} 6$ & $1.374(2)$ & $\mathrm{N} 4-\mathrm{C} 13$ & $1.460(2)$ \\
\hline $\mathrm{C} 2-\mathrm{C} 3$ & $1.374(2)$ & $\mathrm{N} 5-\mathrm{C} 15$ & $1.4638(19)$ \\
\hline $\mathrm{C} 2-\mathrm{H} 2$ & 0.93 & $\mathrm{C} 11-\mathrm{C} 12$ & $1.425(2)$ \\
\hline $\mathrm{C} 3-\mathrm{C} 4$ & $1.367(2)$ & $\mathrm{C} 11-\mathrm{C} 16$ & $1.3841(19)$ \\
\hline $\mathrm{C} 3-\mathrm{H} 3$ & 0.93 & $\mathrm{C} 11-\mathrm{C} 17$ & $1.493(2)$ \\
\hline $\mathrm{C} 4-\mathrm{C} 5$ & $1.3668(15)$ & $\mathrm{C} 12-\mathrm{C} 13$ & $1.409(2)$ \\
\hline $\mathrm{C} 5-\mathrm{C} 6$ & $1.376(2)$ & $\mathrm{C} 13-\mathrm{C} 14$ & $1.376(2)$ \\
\hline $\mathrm{C} 5-\mathrm{H} 5$ & 0.93 & $\mathrm{C} 14-\mathrm{C} 15$ & $1.372(2)$ \\
\hline $\mathrm{C} 6-\mathrm{H} 6$ & 0.93 & $\mathrm{C} 14-\mathrm{H} 14$ & 0.93 \\
\hline $\mathrm{C} 7-\mathrm{C} 8$ & $1.403(2)$ & $\mathrm{C} 15-\mathrm{C} 16$ & $1.379(2)$ \\
\hline $\mathrm{C} 8-\mathrm{C} 9$ & $1.348(3)$ & $\mathrm{C} 16-\mathrm{H} 16$ & 0.93 \\
\hline $\mathrm{C} 8-\mathrm{H} 8$ & 0.93 & & \\
\hline $\mathrm{O} 1-\mathrm{S} 1-\mathrm{O} 2$ & $120.50(6)$ & $\mathrm{C} 8-\mathrm{C} 9-\mathrm{C} 10$ & $133.88(19)$ \\
\hline $\mathrm{O} 1-\mathrm{S} 1-\mathrm{N} 2$ & $108.78(6)$ & $\mathrm{C} 9-\mathrm{C} 10-\mathrm{H} 10 \mathrm{a}$ & 109.47 \\
\hline $\mathrm{O} 2-\mathrm{S} 1-\mathrm{N} 2$ & $104.15(7)$ & $\mathrm{C} 9-\mathrm{C} 10-\mathrm{H} 10 \mathrm{~b}$ & 109.47 \\
\hline $\mathrm{N} 3-\mathrm{O} 3-\mathrm{C} 9$ & $108.94(14)$ & $\mathrm{C} 9-\mathrm{C} 10-\mathrm{H} 10 \mathrm{c}$ & 109.47 \\
\hline $\mathrm{C} 4-\mathrm{N} 1-\mathrm{H} 1 \mathrm{a}$ & $102.59(10)$ & $\mathrm{H} 10 \mathrm{a}-\mathrm{C} 10-\mathrm{H} 10 \mathrm{~b}$ & 109.47 \\
\hline $\mathrm{C} 4-\mathrm{N} 1-\mathrm{H} 1 \mathrm{~b}$ & $107.06(15)$ & $\mathrm{H} 10 \mathrm{a}-\mathrm{C} 10-\mathrm{H} 10 \mathrm{c}$ & 109.47 \\
\hline $\mathrm{C} 4-\mathrm{N} 1-\mathrm{H} 1 \mathrm{c}$ & $107.83(13)$ & $\mathrm{H} 10 \mathrm{~b}-\mathrm{C} 10-\mathrm{H} 10 \mathrm{c}$ & 109.47 \\
\hline $\mathrm{H} 1 \mathrm{a}-\mathrm{N} 1-\mathrm{H} 1 \mathrm{~b}$ & $124.65(16)$ & $\mathrm{C} 17-\mathrm{O} 5-\mathrm{H} 6 \mathrm{a}$ & $105.28(11)$ \\
\hline $\mathrm{H} 1 \mathrm{a}-\mathrm{N} 1-\mathrm{H} 1 \mathrm{c}$ & $103.41(14)$ & $\mathrm{C} 12-\mathrm{O} 6-\mathrm{H} 6 \mathrm{a}$ & $102.01(10)$ \\
\hline $\mathrm{H} 1 \mathrm{~b}-\mathrm{N} 1-\mathrm{H} 1 \mathrm{c}$ & $110.21(14)$ & $\mathrm{O} 7-\mathrm{N} 4-\mathrm{O} 8$ & $123.34(17)$ \\
\hline $\mathrm{S} 1-\mathrm{N} 2-\mathrm{C} 7$ & $124.43(12)$ & $\mathrm{O} 7-\mathrm{N} 4-\mathrm{C} 13$ & $118.74(17)$ \\
\hline $\mathrm{S} 1-\mathrm{N} 2-\mathrm{H} 2 \mathrm{a}$ & $113.65(11)$ & $\mathrm{O} 8-\mathrm{N} 4-\mathrm{C} 13$ & $117.91(17)$ \\
\hline $\mathrm{C} 7-\mathrm{N} 2-\mathrm{H} 2 \mathrm{a}$ & $116.39(12)$ & $\mathrm{O} 9-\mathrm{N} 5-\mathrm{O} 10$ & $124.03(14)$ \\
\hline $\mathrm{O} 3-\mathrm{N} 3-\mathrm{C} 7$ & $104.86(14)$ & $\mathrm{O} 9-\mathrm{N} 5-\mathrm{C} 15$ & $118.56(14)$ \\
\hline $\mathrm{C} 2-\mathrm{C} 1-\mathrm{C} 6$ & $120.38(14)$ & $\mathrm{O} 10-\mathrm{N} 5-\mathrm{C} 15$ & $117.41(15)$ \\
\hline $\mathrm{C} 1-\mathrm{C} 2-\mathrm{C} 3$ & $119.57(16)$ & $\mathrm{C} 12-\mathrm{C} 11-\mathrm{C} 16$ & $120.99(14)$ \\
\hline
\end{tabular}




$\begin{array}{llll}\mathrm{C} 1-\mathrm{C} 2-\mathrm{H} 2 & 120.22 & \mathrm{C} 12-\mathrm{C} 11-\mathrm{C} 17 & 118.93(12) \\ \mathrm{C} 3-\mathrm{C} 2-\mathrm{H} 2 & 120.22 & \mathrm{C} 16-\mathrm{C} 11-\mathrm{C} 17 & 120.07(13) \\ \mathrm{C} 2-\mathrm{C} 3-\mathrm{C} 4 & 119.68(12) & \mathrm{O} 6-\mathrm{C} 12-\mathrm{C} 11 & 121.05(14) \\ \mathrm{C} 2-\mathrm{C} 3-\mathrm{H} 3 & 120.16 & \mathrm{O} 6-\mathrm{C} 12-\mathrm{C} 13 & 122.90(13) \\ \mathrm{C} 4-\mathrm{C} 3-\mathrm{H} 3 & 120.16 & \mathrm{C} 11-\mathrm{C} 12-\mathrm{C} 13 & 116.03(12) \\ \mathrm{N} 1-\mathrm{C} 4-\mathrm{C} 3 & 120.80(10) & \mathrm{N} 4-\mathrm{C} 13-\mathrm{C} 12 & 120.40(13) \\ \mathrm{N} 1-\mathrm{C} 4-\mathrm{C} 5 & 118.09(15) & \mathrm{N} 4-\mathrm{C} 13-\mathrm{C} 14 & 116.57(15) \\ \mathrm{C} 3-\mathrm{C} 4-\mathrm{C} 5 & 121.09(14) & \mathrm{C} 12-\mathrm{C} 13-\mathrm{C} 14 & 123.02(14) \\ \mathrm{C} 4-\mathrm{C} 5-\mathrm{C} 6 & 119.44(16) & \mathrm{C} 13-\mathrm{C} 14-\mathrm{C} 15 & 118.50(15) \\ \mathrm{C} 4-\mathrm{C} 5-\mathrm{H} 5 & 120.28 & \mathrm{C} 13-\mathrm{C} 14-\mathrm{H} 14 & 120.75 \\ \mathrm{C} 6-\mathrm{C} 5-\mathrm{H} 5 & 120.28 & \mathrm{C} 15-\mathrm{C} 14-\mathrm{H} 14 & 120.75 \\ \mathrm{C} 1-\mathrm{C} 6-\mathrm{C} 5 & 119.80(11) & \mathrm{N} 5-\mathrm{C} 15-\mathrm{C} 14 & 117.72(14) \\ \mathrm{C} 1-\mathrm{C} 6-\mathrm{H} 6 & 120.1 & \mathrm{~N} 5-\mathrm{C} 15-\mathrm{C} 16 & 120.43(13) \\ \mathrm{C} 5-\mathrm{C} 6-\mathrm{H} 6 & 120.1 & \mathrm{C} 14-\mathrm{C} 15-\mathrm{C} 16 & 119.61(13) \\ \mathrm{N} 2-\mathrm{C} 7-\mathrm{N} 3 & 117.05(14) & \mathrm{C} 11-\mathrm{C} 16-\mathrm{C} 15 & 120.19 \\ \mathrm{~N} 2-\mathrm{C} 7-\mathrm{C} 8 & 131.01(14) & \mathrm{C} 11-\mathrm{C} 16-\mathrm{H} 16 & 120.19 \\ \mathrm{~N} 3-\mathrm{C} 7-\mathrm{C} 8 & 111.93(15) & \mathrm{C} 15-\mathrm{C} 16-\mathrm{H} 16 & 124.29(16) \\ \mathrm{C} 7-\mathrm{C} 8-\mathrm{C} 9 & 104.20(16) & \mathrm{O} 4-\mathrm{C} 17-\mathrm{O} 5 & 119.59(13) \\ \mathrm{C} 7-\mathrm{C} 8-\mathrm{H} 8 & 127.9 & \mathrm{O} 4-\mathrm{C} 17-\mathrm{C} 11 & 116.11(13) \\ \mathrm{C} 9-\mathrm{C} 8-\mathrm{H} 8 & 127.9 & \mathrm{O} 5-\mathrm{C} 17-\mathrm{C} 11 & 156.58(6) \\ \mathrm{O} 3-\mathrm{C} 9-\mathrm{C} 8 & 110.05(18) & \mathrm{O} 5-\mathrm{H} 6 a-\mathrm{O} 6 & \\ \mathrm{O} 3-\mathrm{C} 9-\mathrm{C} 10 & 116.07(17) & & \end{array}$

Hydrogen-bond geometry $\left(A,{ }^{\circ}\right)$

\begin{tabular}{lllll}
\hline$D-\mathrm{H} \cdots A$ & $D-\mathrm{H}$ & $\mathrm{H} \cdots A$ & $D \cdots A$ & $D-\mathrm{H} \cdots A$ \\
\hline $\mathrm{N} 1-\mathrm{H} 1 a \cdots \mathrm{O} 66^{\mathrm{i}}$ & $1.0015(14)$ & $2.0683(10)$ & $3.0655(17)$ & $173.55(7)$ \\
$\mathrm{N} 1-\mathrm{H} 1 b \cdots \mathrm{N} 3^{\mathrm{ii}}$ & $0.7932(11)$ & $2.2920(11)$ & $3.0393(15)$ & $157.33(11)$ \\
$\mathrm{N} 1-\mathrm{H} 1 c \cdots \mathrm{O} 4^{\mathrm{ii}}$ & $0.8315(15)$ & $1.8318(14)$ & $2.663(2)$ & $177.12(7)$ \\
$\mathrm{N} 2-\mathrm{H} 2 a \cdots \mathrm{O} 5$ & $0.9703(12)$ & $1.8440(10)$ & $2.7852(15)$ & $162.64(9)$ \\
$\mathrm{O} 5-\mathrm{H} 6 a \cdots \mathrm{O} 6$ & $1.2945(12)$ & $1.1837(11)$ & $2.4268(16)$ & $156.58(6)$ \\
$\mathrm{O} 5-\mathrm{H} 6 a \cdots \mathrm{C} 12$ & $1.2945(12)$ & $1.9326(15)$ & $2.7490(19)$ & $115.40(6)$ \\
$\mathrm{O} 6-\mathrm{H} 6 a \cdots \mathrm{O} 5$ & $1.1837(11)$ & $1.2945(12)$ & $2.4268(16)$ & $156.58(6)$ \\
$\mathrm{O} 6-\mathrm{H} 6 a \cdots \mathrm{C} 17$ & $1.1837(11)$ & $2.0485(15)$ & $2.8249(19)$ & $119.42(7)$ \\
\hline
\end{tabular}

Symmetry codes: (i) $-x,-y+2,-z+1$; (ii) $-x+1,-y+2,-z+1$.

2-Isopropyl-6-methyl-4-oxo-3,4-dihydropyrimidin-1-ium 2-carboxy-4,6-dinitrophenolate monohydrate (VABZIJ)

Crystal data

$\mathrm{C}_{8} \mathrm{H}_{13} \mathrm{~N}_{2} \mathrm{O}^{+} \cdot \mathrm{C}_{7} \mathrm{H}_{3} \mathrm{~N}_{2} \mathrm{O}_{7}^{-} \cdot \mathrm{H}_{2} \mathrm{O}$

$M_{r}=398.33$

Triclinic, $P \overline{1}$

Hall symbol: -P 1

$a=6.6691(3) \AA$

$b=11.3831(4) \AA$

$c=12.2900(5) \AA$

$\alpha=89.727(2)^{\circ}$

$\beta=76.771(2)^{\circ}$

$\gamma=76.930(2)^{\circ}$

$V=883.62(6) \AA^{3}$
$Z=2$

$F(000)=416$

$D_{\mathrm{x}}=1.497 \mathrm{Mg} \mathrm{m}^{-3}$

Mo $K \alpha$ radiation, $\lambda=0.71073 \AA$

Cell parameters from 6994 reflections

$\theta=2.4-31.6^{\circ}$

$\mu=0.13 \mathrm{~mm}^{-1}$

$T=100 \mathrm{~K}$

Block, yellow

$0.52 \times 0.13 \times 0.10 \mathrm{~mm}$ 


\section{Data collection}

Bruker SMART APEXII CCD area-detector diffractometer

Radiation source: fine-focus sealed tube Graphite monochromator $\varphi$ and $\omega$ scans Absorption correction: multi-scan (SADABS; Bruker, 2009)

$T_{\min }=0.937, T_{\max }=0.987$

\section{Refinement}

Refinement on $F^{2}$

$R[F>3 \sigma(F)]=0.038$

$w R(F)=0.086$

$S=1.77$

4061 reflections

258 parameters

0 restraints

52 constraints

Primary atom site location: structure-invariant direct methods
17014 measured reflections

4061 independent reflections

3042 reflections with $I>3 \sigma(I)$

$R_{\text {int }}=0.030$

$\theta_{\max }=27.5^{\circ}, \theta_{\min }=1.7^{\circ}$

$h=-8 \rightarrow 8$

$k=-14 \rightarrow 12$

$l=-15 \rightarrow 15$

Secondary atom site location: difference Fourier map

Hydrogen site location: difference Fourier map

$\mathrm{H}$ atoms treated by a mixture of independent and constrained refinement

Weighting scheme based on measured s.u.'s $w=$ $1 /\left(\sigma^{2}(I)+0.0004 I^{2}\right)$

$(\Delta / \sigma)_{\max }=0.014$

$\Delta \rho_{\max }=0.46$ e $\AA^{-3}$

$\Delta \rho_{\text {min }}=-0.23$ e $\AA^{-3}$

Special details

Experimental. The crystal was placed in the cold stream of an Oxford Cryosystems Cobra open-flow nitrogen cryostat (Cosier \& Glazer, 1986) operating at 100.0 (1) K.

Geometry. All s.u.'s (except the s.u. in the dihedral angle between two 1.s. planes) are estimated using the full covariance matrix. The cell s.u.'s are taken into account individually in the estimation of s.u.'s in distances, angles and torsion angles; correlations between s.u.'s in cell parameters are only used when they are defined by crystal symmetry. An approximate (isotropic) treatment of cell s.u.'s is used for estimating s.u.'s involving l.s. planes.

Refinement. Refinement of $\mathrm{F}^{2}$ against ALL reflections. The weighted R-factor $\mathrm{wR}$ and goodness of fit $\mathrm{S}$ are based on $\mathrm{F}^{2}$, conventional R-factors $\mathrm{R}$ are based on $\mathrm{F}$, with $\mathrm{F}$ set to zero for negative $\mathrm{F}^{2}$. The threshold expression of $\mathrm{F}^{2}>2 \sigma\left(\mathrm{F}^{2}\right)$ is used only for calculating R-factors(gt) etc. and is not relevant to the choice of reflections for refinement. R-factors based on $\mathrm{F}^{2}$ are statistically about twice as large as those based on F, and R- factors based on ALL data will be even larger.

Number of fixed parameters: 15

Fractional atomic coordinates and isotropic or equivalent isotropic displacement parameters $\left(\AA^{2}\right)$

\begin{tabular}{lllll}
\hline & $x$ & $y$ & $z$ & $U_{\text {iso }} * / U_{\text {eq }}$ \\
\hline O1 & $0.30546(16)$ & $-0.17099(9)$ & $0.14963(8)$ & $0.0232(4)$ \\
O2 & $0.56653(17)$ & $-0.35718(9)$ & $0.01361(9)$ & $0.0316(4)$ \\
O3 & $0.48656(18)$ & $-0.36691(9)$ & $-0.14697(9)$ & $0.0311(4)$ \\
O4 & $0.36254(16)$ & $-0.00313(9)$ & $-0.33889(8)$ & $0.0235(4)$ \\
O5 & $0.23753(16)$ & $0.16995(9)$ & $-0.24573(8)$ & $0.0255(4)$ \\
O6 & $0.07250(15)$ & $0.19153(8)$ & $0.16330(8)$ & $0.0204(4)$ \\
O7 & $0.14533(16)$ & $0.02234(9)$ & $0.25238(8)$ & $0.0215(4)$ \\
O8 & $0.31889(16)$ & $0.38510(9)$ & $0.41956(8)$ & $0.0221(4)$ \\
N1 & $0.30174(18)$ & $0.05930(11)$ & $-0.25094(10)$ & $0.0185(4)$ \\
N2 & $0.48606(19)$ & $-0.31064(11)$ & $-0.06125(10)$ & $0.0213(4)$ \\
N3 & $0.11898(17)$ & $0.64252(10)$ & $0.66449(9)$ & $0.0147(4)$ \\
N4 & $0.23841(17)$ & $0.58259(10)$ & $0.47936(9)$ & $0.0144(4)$ \\
C1 & $0.3103(2)$ & $-0.11910(12)$ & $0.05547(11)$ & $0.0151(5)$ \\
C2 & $0.3895(2)$ & $-0.18160(12)$ & $-0.05145(11)$ & $0.0154(5)$
\end{tabular}




\begin{tabular}{|c|c|c|c|c|}
\hline $\mathrm{C} 3$ & $0.3838(2)$ & $-0.12419(13)$ & $-0.15034(12)$ & $0.0160(5)$ \\
\hline $\mathrm{H} 3 \mathrm{a}$ & 0.432743 & -0.167918 & -0.218686 & $0.0192 *$ \\
\hline $\mathrm{C} 4$ & $0.3044(2)$ & $-0.00126(13)$ & $-0.14615(11)$ & $0.0150(5)$ \\
\hline $\mathrm{C} 5$ & $0.2281(2)$ & $0.06626(13)$ & $-0.04539(11)$ & $0.0148(5)$ \\
\hline H5a & 0.176964 & 0.149404 & -0.044656 & $0.0177 *$ \\
\hline C6 & $0.2290(2)$ & $0.00871(12)$ & $0.05379(11)$ & $0.0140(5)$ \\
\hline $\mathrm{C} 7$ & $0.1429(2)$ & $0.08121(13)$ & $0.16123(11)$ & $0.0159(5)$ \\
\hline $\mathrm{C} 8$ & $0.2598(2)$ & $0.45829(12)$ & $0.49910(12)$ & $0.0158(5)$ \\
\hline C9 & $0.2070(2)$ & $0.43367(13)$ & $0.61582(11)$ & $0.0160(5)$ \\
\hline H9a & 0.221191 & 0.354014 & 0.636387 & $0.0192 *$ \\
\hline $\mathrm{C} 10$ & $0.1376(2)$ & $0.52320(12)$ & $0.69587(11)$ & $0.0157(5)$ \\
\hline $\mathrm{C} 11$ & $0.1694(2)$ & $0.67095(12)$ & $0.55875(11)$ & $0.0141(5)$ \\
\hline $\mathrm{C} 12$ & $0.1445(2)$ & $0.80006(12)$ & $0.52928(11)$ & $0.0153(5)$ \\
\hline $\mathrm{H} 12 \mathrm{a}$ & 0.110348 & 0.85057 & 0.598371 & $0.0184^{*}$ \\
\hline $\mathrm{C} 13$ & $-0.0374(2)$ & 0.83589 (13) & 0.47019 (13) & 0.0224 \\
\hline H13a & -0.054927 & 0.919318 & 0.452627 & $0.0335^{*}$ \\
\hline $\mathrm{H} 13 \mathrm{~b}$ & -0.165759 & 0.824046 & 0.518462 & $0.0335 *$ \\
\hline $\mathrm{H} 13 \mathrm{c}$ & -0.005734 & 0.786763 & 0.402409 & $0.0335^{*}$ \\
\hline $\mathrm{C} 14$ & $0.3509(2)$ & $0.82298(12)$ & $0.45745(12)$ & 0.0195 \\
\hline $\mathrm{H} 14 \mathrm{a}$ & 0.461527 & 0.800548 & 0.496897 & $0.0292 *$ \\
\hline $\mathrm{H} 14 \mathrm{~b}$ & 0.331515 & 0.907081 & 0.442205 & $0.0292 *$ \\
\hline $\mathrm{H} 14 \mathrm{c}$ & 0.389104 & 0.775688 & 0.38823 & $0.0292 *$ \\
\hline $\mathrm{C} 15$ & $0.0772(2)$ & $0.50600(13)$ & $0.81821(12)$ & $0.0221(5)$ \\
\hline H15a & -0.068894 & 0.545223 & 0.847126 & $0.0332 *$ \\
\hline $\mathrm{H} 15 \mathrm{~b}$ & 0.163895 & 0.540389 & 0.855439 & $0.0332 *$ \\
\hline $\mathrm{H} 15 \mathrm{c}$ & 0.097793 & 0.421295 & 0.830953 & $0.0332 *$ \\
\hline O1w & $0.32852(17)$ & $0.60955(9)$ & $0.25340(8)$ & $0.0272(4)$ \\
\hline $\mathrm{H} 1 \mathrm{n} 3$ & 0.061415 & 0.706878 & 0.722609 & $0.037(5)^{*}$ \\
\hline $\mathrm{H} 1 \mathrm{n} 4$ & 0.270467 & 0.602234 & 0.406704 & $0.034(5)^{*}$ \\
\hline $\mathrm{H} 2 \mathrm{w} 1$ & 0.339729 & 0.675124 & 0.210779 & $0.053(6)^{*}$ \\
\hline H1w1 & 0.381611 & 0.539911 & 0.20932 & $0.055(6)^{*}$ \\
\hline $\mathrm{H} 7$ & 0.211364 & -0.063754 & 0.220331 & $0.078(7)^{*}$ \\
\hline
\end{tabular}

Atomic displacement parameters $\left(\AA^{2}\right)$

\begin{tabular}{lllllll}
\hline & $U^{11}$ & $U^{22}$ & $U^{33}$ & $U^{12}$ & $U^{13}$ & $U^{23}$ \\
\hline O1 & $0.0305(6)$ & $0.0178(6)$ & $0.0185(6)$ & $-0.0038(5)$ & $-0.0018(5)$ & $0.0031(4)$ \\
O2 & $0.0431(7)$ & $0.0219(6)$ & $0.0232(6)$ & $0.0045(5)$ & $-0.0063(5)$ & $0.0044(5)$ \\
O3 & $0.0489(7)$ & $0.0181(6)$ & $0.0229(6)$ & $-0.0036(5)$ & $-0.0053(5)$ & $-0.0086(5)$ \\
O4 & $0.0291(6)$ & $0.0274(6)$ & $0.0138(6)$ & $-0.0083(5)$ & $-0.0030(5)$ & $-0.0009(5)$ \\
O5 & $0.0347(6)$ & $0.0178(6)$ & $0.0235(6)$ & $-0.0045(5)$ & $-0.0072(5)$ & $0.0058(5)$ \\
O6 & $0.0244(6)$ & $0.0142(6)$ & $0.0187(6)$ & $-0.0013(4)$ & $-0.0004(4)$ & $-0.0029(4)$ \\
O7 & $0.0284(6)$ & $0.0175(6)$ & $0.0140(5)$ & $-0.0009(5)$ & $0.0003(4)$ & $-0.0003(4)$ \\
O8 & $0.0321(6)$ & $0.0137(5)$ & $0.0176(6)$ & $-0.0026(5)$ & $-0.0027(5)$ & $-0.0025(4)$ \\
N1 & $0.0182(6)$ & $0.0202(7)$ & $0.0186(7)$ & $-0.0071(6)$ & $-0.0047(5)$ & $0.0034(6)$ \\
N2 & $0.0240(7)$ & $0.0161(7)$ & $0.0198(7)$ & $-0.0035(6)$ & $0.0019(6)$ & $0.0001(6)$ \\
N3 & $0.0168(6)$ & $0.0123(6)$ & $0.0136(6)$ & $-0.0024(5)$ & $-0.0017(5)$ & $-0.0006(5)$ \\
N4 & $0.0172(6)$ & $0.0117(6)$ & $0.0129(6)$ & $-0.0026(5)$ & $-0.0014(5)$ & $0.0011(5)$
\end{tabular}


Geometric parameters $\left(\AA,{ }^{\circ}\right)$

\begin{tabular}{|c|c|c|c|}
\hline $\mathrm{O} 1-\mathrm{C} 1$ & $1.2939(17)$ & $\mathrm{C} 4-\mathrm{C} 5$ & $1.3881(18)$ \\
\hline $\mathrm{O} 1-\mathrm{H} 7$ & $1.4329(9)$ & $\mathrm{C} 5-\mathrm{H} 5 \mathrm{a}$ & 0.93 \\
\hline $\mathrm{O} 2-\mathrm{N} 2$ & $1.2279(17)$ & $\mathrm{C} 5-\mathrm{C} 6$ & $1.3816(19)$ \\
\hline $\mathrm{O} 3-\mathrm{N} 2$ & $1.2346(17)$ & $\mathrm{C} 6-\mathrm{C} 7$ & $1.4844(18)$ \\
\hline $\mathrm{O} 4-\mathrm{N} 1$ & $1.2304(15)$ & $\mathrm{C} 8-\mathrm{C} 9$ & $1.439(2)$ \\
\hline $\mathrm{O} 5-\mathrm{N} 1$ & $1.2321(15)$ & $\mathrm{C} 9-\mathrm{H} 9 \mathrm{a}$ & 0.93 \\
\hline $\mathrm{O} 6-\mathrm{C} 7$ & $1.2355(16)$ & $\mathrm{C} 9-\mathrm{C} 10$ & 1.3449 (19) \\
\hline $\mathrm{O} 7-\mathrm{C} 7$ & $1.3040(17)$ & $\mathrm{C} 10-\mathrm{C} 15$ & 1.4889 (19) \\
\hline $\mathrm{O} 7-\mathrm{H} 7$ & $1.0191(9)$ & $\mathrm{C} 11-\mathrm{C} 12$ & $1.4936(19)$ \\
\hline $\mathrm{O} 8-\mathrm{C} 8$ & $1.2198(17)$ & $\mathrm{C} 12-\mathrm{H} 12 \mathrm{a}$ & 0.98 \\
\hline $\mathrm{N} 1-\mathrm{C} 4$ & $1.4597(18)$ & $\mathrm{C} 12-\mathrm{C} 13$ & $1.531(2)$ \\
\hline $\mathrm{N} 2-\mathrm{C} 2$ & $1.4576(17)$ & $\mathrm{C} 12-\mathrm{C} 14$ & $1.5318(19)$ \\
\hline N3- 110 & $1.3963(18)$ & $\mathrm{C} 13-\mathrm{H} 13 \mathrm{a}$ & 0.96 \\
\hline N3-C11 & $1.3236(17)$ & $\mathrm{C} 13-\mathrm{H} 13 \mathrm{~b}$ & 0.96 \\
\hline $\mathrm{N} 3-\mathrm{H} 1 \mathrm{n} 3$ & $0.9729(11)$ & $\mathrm{C} 13-\mathrm{H} 13 \mathrm{c}$ & 0.96 \\
\hline N4-C8 & $1.4152(18)$ & $\mathrm{C} 14-\mathrm{H} 14 \mathrm{a}$ & 0.96 \\
\hline $\mathrm{N} 4-\mathrm{C} 11$ & $1.3303(17)$ & $\mathrm{C} 14-\mathrm{H} 14 \mathrm{~b}$ & 0.96 \\
\hline $\mathrm{N} 4-\mathrm{H} 1 \mathrm{n} 4$ & $0.9085(11)$ & $\mathrm{C} 14-\mathrm{H} 14 \mathrm{c}$ & 0.96 \\
\hline $\mathrm{C} 1-\mathrm{C} 2$ & $1.4259(18)$ & $\mathrm{C} 15-\mathrm{H} 15 \mathrm{a}$ & 0.96 \\
\hline $\mathrm{C} 1-\mathrm{C} 6$ & 1.4349 (19) & $\mathrm{C} 15-\mathrm{H} 15 \mathrm{~b}$ & 0.96 \\
\hline $\mathrm{C} 2-\mathrm{C} 3$ & $1.381(2)$ & $\mathrm{C} 15-\mathrm{H} 15 \mathrm{c}$ & 0.96 \\
\hline $\mathrm{C} 3-\mathrm{H} 3 \mathrm{a}$ & 0.93 & $\mathrm{O} 1 \mathrm{w}-\mathrm{H} 2 \mathrm{w} 1$ & $0.9169(10)$ \\
\hline $\mathrm{C} 3-\mathrm{C} 4$ & $1.3763(19)$ & $\mathrm{O} 1 \mathrm{w}-\mathrm{H} 1 \mathrm{w} 1$ & $0.9146(10)$ \\
\hline $\mathrm{C} 1-\mathrm{O} 1-\mathrm{H} 7$ & $96.54(8)$ & $\mathrm{N} 4-\mathrm{C} 8-\mathrm{C} 9$ & $113.60(12)$ \\
\hline $\mathrm{C} 7-\mathrm{O} 7-\mathrm{H} 7$ & $101.27(9)$ & $\mathrm{C} 8-\mathrm{C} 9-\mathrm{H} 9 \mathrm{a}$ & 119.24 \\
\hline $\mathrm{O} 4-\mathrm{N} 1-\mathrm{O} 5$ & $124.04(12)$ & $\mathrm{C} 8-\mathrm{C} 9-\mathrm{C} 10$ & $121.51(13)$ \\
\hline $\mathrm{O} 4-\mathrm{N} 1-\mathrm{C} 4$ & $118.12(11)$ & $\mathrm{H} 9 \mathrm{a}-\mathrm{C} 9-\mathrm{C} 10$ & 119.24 \\
\hline $\mathrm{O} 5-\mathrm{N} 1-\mathrm{C} 4$ & $117.84(11)$ & $\mathrm{N} 3-\mathrm{C} 10-\mathrm{C} 9$ & $118.95(12)$ \\
\hline
\end{tabular}




$\begin{array}{ll}\mathrm{O} 2-\mathrm{N} 2-\mathrm{O} 3 & 123.42(12) \\ \mathrm{O} 2-\mathrm{N} 2-\mathrm{C} 2 & 119.16(12) \\ \mathrm{O} 3-\mathrm{N} 2-\mathrm{C} 2 & 117.39(12) \\ \mathrm{C} 10-\mathrm{N} 3-\mathrm{C} 11 & 122.45(11) \\ \mathrm{C} 10-\mathrm{N} 3-\mathrm{H} 1 \mathrm{n} 3 & 118.37(11) \\ \mathrm{C} 11-\mathrm{N} 3-\mathrm{H} 1 \mathrm{n} 3 & 119.14(11) \\ \mathrm{C} 8-\mathrm{N} 4-\mathrm{C} 11 & 124.69(12) \\ \mathrm{C} 8-\mathrm{N} 4-\mathrm{H} 1 \mathrm{n} 4 & 116.55(11) \\ \mathrm{C} 11-\mathrm{N} 4-\mathrm{H} 1 \mathrm{n} 4 & 118.72(12) \\ \mathrm{O} 1-\mathrm{C} 1-\mathrm{C} 2 & 124.06(12) \\ \mathrm{O} 1-\mathrm{C} 1-\mathrm{C} 6 & 120.40(11) \\ \mathrm{C} 2-\mathrm{C} 1-\mathrm{C} 6 & 115.53(12) \\ \mathrm{N} 2-\mathrm{C} 2-\mathrm{C} 1 & 120.85(12) \\ \mathrm{N} 2-\mathrm{C} 2-\mathrm{C} 3 & 116.56(11) \\ \mathrm{C} 1-\mathrm{C} 2-\mathrm{C} 3 & 122.58(12) \\ \mathrm{C} 2-\mathrm{C} 3-\mathrm{H} 3 \mathrm{a} & 120.52 \\ \mathrm{C} 2-\mathrm{C} 3-\mathrm{C} 4 & 118.97(12) \\ \mathrm{H} 3 \mathrm{a}-\mathrm{C} 3-\mathrm{C} 4 & 120.52 \\ \mathrm{~N} 1-\mathrm{C} 4-\mathrm{C} 3 & 118.77(12) \\ \mathrm{N} 1-\mathrm{C} 4-\mathrm{C} 5 & 119.38(12) \\ \mathrm{C} 3-\mathrm{C} 4-\mathrm{C} 5 & 121.85(13) \\ \mathrm{C} 4-\mathrm{C} 5-\mathrm{H} 5 \mathrm{a} & 120.33 \\ \mathrm{C} 4-\mathrm{C} 5-\mathrm{C} 6 & 119.34(12) \\ \mathrm{H} 5 \mathrm{a}-\mathrm{C} 5-\mathrm{C} 6 & 120.33 \\ \mathrm{C} 1-\mathrm{C} 6-\mathrm{C} 5 & 121.71(12) \\ \mathrm{C} 1-\mathrm{C} 6-\mathrm{C} 7 & 119.30(12) \\ \mathrm{C} 5-\mathrm{C} 6-\mathrm{C} 7 & 118.99(12) \\ \mathrm{O} 6-\mathrm{C} 7-\mathrm{O} 7 & 122.20(12) \\ \mathrm{O} 6-\mathrm{C} 7-\mathrm{C} 6 & 121.26(12) \\ \mathrm{O} 7-\mathrm{C} 7-\mathrm{C} 6 & 116.54(12) \\ \mathrm{O} 8-\mathrm{C} 8-\mathrm{N} 4 & 119.14(12) \\ \mathrm{O} 8-\mathrm{C} 8-\mathrm{C} 9 & 127.26(13) \\ & \end{array}$

$\begin{array}{ll}\text { N3-C10-C15 } & 116.00(11) \\ \text { C9-C10-C15 } & 125.06(13) \\ \text { N3-C11-N4 } & 118.76(12) \\ \text { N3-C11-C12 } & 120.45(12) \\ \text { N4-C11-C12 } & 120.78(12) \\ \text { C11-C12-H12a } & 108.71 \\ \text { C11-C12-C13 } & 109.61(13) \\ \text { C11-C12-C14 } & 111.42(10) \\ \text { H12a-C12-C13 } & 108.78 \\ \text { H12a-C12-C14 } & 106.87 \\ \text { C13-C12-C14 } & 111.36(12) \\ \text { C12-C13-H13a } & 109.47 \\ \text { C12-C13-H13b } & 109.47 \\ \text { C12-C13-H13c } & 109.47 \\ \text { H13a-C13-H13b } & 109.47 \\ \text { H13a-C13-H13c } & 109.47 \\ \text { H13b-C13-H13c } & 109.47 \\ \text { C12-C14-H14a } & 109.47 \\ \text { C12-C14-H14b } & 109.47 \\ \text { C12-C14-H14c } & 109.47 \\ \text { H14a-C14-H14b } & 109.47 \\ \text { H14a-C14-H14c } & 109.47 \\ \text { H14b-C14-H14c } & 109.47 \\ \text { C10-C15-H15a } & 109.47 \\ \text { C10-C15-H15b } & 109.47 \\ \text { C10-C15-H15c } & 109.47 \\ \text { H15a-C15-H15b } & 109.47 \\ \text { H15a-C15-H15c } & 109.47 \\ \text { H15b-C15-H15c } & 109.47 \\ \text { H2w1-O1w-H1w1 } & 110.00(10) \\ \text { O1-H7-O7 } & 165.94(7) \\ & \end{array}$

Hydrogen-bond geometry $\left(A,{ }^{\circ}\right)$

\begin{tabular}{lllll}
\hline$D-\mathrm{H} \cdots A$ & $D-\mathrm{H}$ & $\mathrm{H} \cdots A$ & $D \cdots A$ & $D-\mathrm{H}^{\cdots} A$ \\
\hline $\mathrm{C} 12-\mathrm{H} 12 a \cdots \mathrm{O}^{\mathrm{i}}$ & 0.98 & 2.42 & $3.3068(15)$ & 151 \\
$\mathrm{~N} 3-\mathrm{H} 1 n 3 \cdots 6^{\mathrm{i}}$ & $0.9729(11)$ & $1.7539(9)$ & $2.7182(14)$ & $170.48(8)$ \\
$\mathrm{N} 4-\mathrm{H} 1 n 4 \cdots \mathrm{O} 1 w$ & $0.9085(11)$ & $1.8401(10)$ & $2.7348(15)$ & $167.76(8)$ \\
$\mathrm{O} 1 w-\mathrm{H} 2 w 1 \cdots \mathrm{O} 1^{\mathrm{ii}}$ & $0.9169(10)$ & $1.8895(10)$ & $2.7886(14)$ & $166.21(6)$ \\
$\mathrm{O} 1 w-\mathrm{H} 1 w 1 \cdots 3^{i i i}$ & $0.9146(10)$ & $2.0399(10)$ & $2.9352(14)$ & $165.84(7)$ \\
$\mathrm{O} 7-\mathrm{H} 7 \cdots \mathrm{O} 1$ & $1.0191(9)$ & $1.4329(9)$ & $2.4340(13)$ & $165.94(7)$ \\
\hline
\end{tabular}

Symmetry codes: (i) $-x,-y+1,-z+1$; (ii) $x, y+1, z$; (iii) $-x+1,-y,-z$. 
1-Aza-8-azoniabicyclo[5.4.0] undec-7-ene 4-aminobenzoate (WADXOR)

Crystal data

$\mathrm{C}_{9} \mathrm{H}_{17} \mathrm{~N}_{2}^{+} \cdot \mathrm{C}_{7} \mathrm{H}_{3} \mathrm{~N}_{2} \mathrm{O}_{7}^{-}$

$M_{r}=380.35$

Monoclinic, $P 2{ }_{1} / n$

Hall symbol: -P 2yabc

$a=6.1537(3) \AA$

$b=19.1541(14) \AA$

$c=14.5527(11) \AA$

$\beta=98.343(6)^{\circ}$

$V=1697.2(2) \AA^{3}$

$Z=4$

\section{Data collection}

Oxford Diffraction Gemini-S CCD-detector diffractometer

Graphite monochromator

Detector resolution: 16.067 pixels $\mathrm{mm}^{-1}$

$\omega$ scans

Absorption correction: multi-scan

(CrysAlis PRO; Agilent, 2014)

$T_{\min }=0.920, T_{\max }=0.990$

\section{Refinement}

Refinement on $F^{2}$

Least-squares matrix: full

$R\left[F^{2}>2 \sigma\left(F^{2}\right)\right]=0.046$

$w R\left(F^{2}\right)=0.095$

$S=1.33$

3339 reflections

268 parameters

2 restraints

98 constraints

Primary atom site location: structure-invariant direct methods
$F(000)=800$

$D_{\mathrm{x}}=1.489 \mathrm{Mg} \mathrm{m}^{-3}$

Mo $K \alpha$ radiation, $\lambda=0.71073 \AA$

Cell parameters from 1891 reflections

$\theta=3.5-26.6^{\circ}$

$\mu=0.12 \mathrm{~mm}^{-1}$

$T=200 \mathrm{~K}$

Needle, yellow

$0.30 \times 0.13 \times 0.10 \mathrm{~mm}$

7800 measured reflections

3339 independent reflections

1976 reflections with $I>3 \sigma(I)$

$R_{\text {int }}=0.034$

$\theta_{\text {max }}=26.0^{\circ}, \theta_{\min }=3.4^{\circ}$

$h=-7 \rightarrow 7$

$k=-23 \rightarrow 23$

$l=-17 \rightarrow 17$

Secondary atom site location: difference Fourier map

Hydrogen site location: difference Fourier map

$\mathrm{H}$ atoms treated by a mixture of independent and constrained refinement

Weighting scheme based on measured s.u.'s $w=$ $1 /\left(\sigma^{2}(I)+0.0004 I^{2}\right)$

$(\Delta / \sigma)_{\max }=0.005$

$\Delta \rho_{\max }=0.36$ e $\AA^{-3}$

$\Delta \rho_{\min }=-0.24$ e $\AA^{-3}$

Extinction correction: B-C type 1 Lorentzian isotropic (Becker \& Coppens, 1974)

Extinction coefficient: 2200 (700)

\section{Special details}

Geometry. Bond distances, angles etc. have been calculated using the rounded fractional coordinates. All su's are estimated from the variances of the (full) variance-covariance matrix. The cell esds are taken into account in the estimation of distances, angles and torsion angles

Refinement. Refinement of $\mathrm{F}^{2}$ against ALL reflections. The weighted R-factor wR and goodness of fit $\mathrm{S}$ are based on $\mathrm{F}^{2}$, conventional R-factors $R$ are based on $F$, with $F$ set to zero for negative $F^{2}$. The threshold expression of $F^{2}>2 \operatorname{sigma}\left(F^{2}\right)$ is used only for calculating R-factors(gt) etc. and is not relevant to the choice of reflections for refinement. R-factors based on $\mathrm{F}^{2}$ are statistically about twice as large as those based on F, and R- factors based on ALL data will be even larger.

Number of fixed parameters : 10

Fractional atomic coordinates and isotropic or equivalent isotropic displacement parameters $\left(\AA^{2}\right)$

\begin{tabular}{llllll}
\hline & $x$ & $y$ & $z$ & $U_{\text {iso }} * / U_{\text {eq }}$ & Occ. $(<1)$ \\
\hline O2b & $0.8418(3)$ & $0.56169(11)$ & $0.78950(14)$ & $0.0440(8)$ & $0.727(4)$ \\
H61b & 0.852107 & 0.560819 & 0.76707 & $0.0359^{*}$ & $0.273(4)$
\end{tabular}




\begin{tabular}{|c|c|c|c|c|c|}
\hline $\mathrm{O} 21 \mathrm{~b}$ & $0.7735(8)$ & $0.6569(3)$ & $0.4912(4)$ & $0.048(2)$ & $0.273(4)$ \\
\hline $\mathrm{H} 6 \mathrm{~b}$ & 0.805712 & 0.644967 & 0.507207 & $0.0328 *$ & $0.727(4)$ \\
\hline O11b & $0.5083(2)$ & $0.68884(8)$ & $0.59278(11)$ & $0.0444(6)$ & \\
\hline $\mathrm{O} 12 \mathrm{~b}$ & $0.5447(2)$ & $0.64534(8)$ & $0.73586(12)$ & $0.0524(7)$ & \\
\hline $\mathrm{O} 31 \mathrm{~b}$ & $1.1108(3)$ & $0.45706(10)$ & $0.81717(14)$ & $0.0829(9)$ & \\
\hline $\mathrm{O} 32 \mathrm{~b}$ & $1.4073(3)$ & $0.47245(10)$ & $0.75770(13)$ & $0.0775(8)$ & \\
\hline O51b & $1.3288(3)$ & $0.55869(9)$ & $0.44602(12)$ & $0.0603(7)$ & \\
\hline $\mathrm{O} 52 \mathrm{~b}$ & $1.0707(3)$ & $0.63212(10)$ & $0.39870(12)$ & $0.0680(8)$ & \\
\hline $\mathrm{N} 3 \mathrm{~b}$ & $1.2111(3)$ & $0.48284(10)$ & $0.76049(14)$ & $0.0472(8)$ & \\
\hline $\mathrm{N} 5 \mathrm{~b}$ & $1.1654(3)$ & $0.59180(10)$ & $0.45701(14)$ & $0.0418(7)$ & \\
\hline $\mathrm{C} 1 \mathrm{~b}$ & 0.8002 & $0.60956(10)$ & $0.63903(15)$ & $0.0264(7)$ & \\
\hline $\mathrm{C} 2 \mathrm{~b}$ & 0.9062 & $0.56604(11)$ & $0.70954(15)$ & $0.0299(7)$ & \\
\hline $\mathrm{C} 3 \mathrm{~b}$ & 1.0952 (3) & $0.53063(10)$ & $0.69140(15)$ & $0.0309(7)$ & \\
\hline $\mathrm{C} 4 \mathrm{~b}$ & $1.1814(3)$ & $0.53946(10)$ & $0.61042(15)$ & $0.0309(7)$ & \\
\hline $\mathrm{C} 5 \mathrm{~b}$ & $1.0736(3)$ & $0.58234(10)$ & $0.54290(14)$ & $0.0271(7)$ & \\
\hline $\mathrm{C} 6 \mathrm{~b}$ & $0.8810(3)$ & $0.61681(10)$ & $0.55538(15)$ & $0.0273(7)$ & \\
\hline $\mathrm{C} 11 \mathrm{~b}$ & $0.6030(3)$ & $0.65080(11)$ & $0.65583(18)$ & $0.0351(8)$ & \\
\hline N1a & $-0.1521(2)$ & $0.82026(8)$ & $0.63826(12)$ & $0.0293(6)$ & \\
\hline N8a & $0.1721(2)$ & $0.76019(9)$ & $0.67283(12)$ & $0.0373(7)$ & \\
\hline $\mathrm{C} 2 \mathrm{a}$ & -0.3261 & $0.85089(11)$ & $0.57022(15)$ & $0.0370(8)$ & \\
\hline $\mathrm{C} 3 \mathrm{a}$ & -0.2602 & $0.91805(11)$ & $0.52679(15)$ & $0.0401(8)$ & \\
\hline $\mathrm{C} 4 \mathrm{a}$ & -0.1188 & $0.90791(11)$ & $0.45061(16)$ & $0.0417(8)$ & \\
\hline $\mathrm{C} 5 \mathrm{a}$ & 0.0933 & $0.86805(11)$ & $0.48032(14)$ & $0.0401(8)$ & \\
\hline C6a & 0.0611 & 0.79367 (11) & $0.51423(14)$ & $0.0344(8)$ & \\
\hline $\mathrm{C} 7 \mathrm{a}$ & $0.0226(3)$ & $0.79087(10)$ & $0.61288(14)$ & $0.0268(7)$ & \\
\hline $\mathrm{C} 9 \mathrm{a}$ & $0.1394(8)$ & $0.7480(3)$ & $0.7694(4)$ & 0.0377 (17) & $0.687(4)$ \\
\hline $\mathrm{C} 10 \mathrm{a}$ & $0.0234(5)$ & $0.8112(2)$ & $0.8006(2)$ & $0.0388(12)$ & $0.687(4)$ \\
\hline C11a & -0.1865 (3) & $0.82334(13)$ & $0.73606(16)$ & $0.0375(8)$ & \\
\hline $\mathrm{C} 13 \mathrm{a}$ & $0.192(2)$ & $0.7733(6)$ & $0.7752(10)$ & 0.0377 (17) & $0.313(4)$ \\
\hline $\mathrm{C} 12 \mathrm{a}$ & $-0.0453(12)$ & $0.7700(5)$ & $0.7962(6)$ & $0.0388(12)$ & $0.313(4)$ \\
\hline $\mathrm{H} 4 \mathrm{~b}$ & 1.313123 & 0.516393 & 0.601144 & $0.0371^{*}$ & \\
\hline $\mathrm{H} 8 \mathrm{a}$ & 0.304017 & 0.743029 & 0.652706 & $0.073(8)^{*}$ & \\
\hline H10a & 0.119515 & 0.852593 & 0.800333 & $0.0466^{*}$ & 0.686 \\
\hline $\mathrm{H} 21 \mathrm{a}$ & -0.458393 & 0.859249 & 0.600103 & $0.0444^{*}$ & \\
\hline $\mathrm{H} 22 \mathrm{a}$ & -0.373911 & 0.816372 & 0.520773 & $0.0444^{*}$ & \\
\hline H31a & -0.393523 & 0.944805 & 0.502041 & $0.0481^{*}$ & \\
\hline $\mathrm{H} 32 \mathrm{a}$ & -0.182575 & 0.948658 & 0.575716 & $0.0481^{*}$ & \\
\hline H41a & -0.084564 & 0.95395 & 0.425285 & $0.0501 *$ & \\
\hline $\mathrm{H} 42 \mathrm{a}$ & -0.20539 & 0.883982 & 0.397103 & $0.0501 *$ & \\
\hline H51a & 0.180506 & 0.866759 & 0.428286 & $0.0482 *$ & \\
\hline H52a & 0.186838 & 0.894538 & 0.52943 & $0.0482 *$ & \\
\hline H61a & 0.191356 & 0.765112 & 0.506561 & $0.0413 *$ & \\
\hline H62a & -0.063947 & 0.77162 & 0.474177 & $0.0413 *$ & \\
\hline H91a & 0.046176 & 0.706149 & 0.772504 & $0.0452 *$ & $0.687(4)$ \\
\hline H92a & 0.283833 & 0.74265 & 0.808712 & $0.0452 *$ & $0.687(4)$ \\
\hline H11a & -0.009873 & 0.803439 & 0.864351 & $0.0466^{*}$ & 0.686 \\
\hline $\mathrm{H} 12 \mathrm{a}$ & $-0.318(2)$ & $0.7965(9)$ & $0.7425(13)$ & $0.045^{*}$ & \\
\hline $\mathrm{H} 13 \mathrm{a}$ & -0.230 & $0.8711(6)$ & $0.7485(13)$ & $0.045^{*}$ & \\
\hline
\end{tabular}




\begin{tabular}{llllll} 
H14a & -0.105082 & 0.7226 & 0.782436 & $0.0466^{*}$ & $0.313(4)$ \\
H15a & -0.047311 & 0.780768 & 0.862598 & $0.0466^{*}$ & $0.313(4)$ \\
H16a & 0.27893 & 0.735519 & 0.809131 & $0.0452^{*}$ & $0.313(4)$ \\
H17a & 0.251697 & 0.820686 & 0.789237 & $0.0452^{*}$ & $0.313(4)$ \\
H21b & 0.621837 & 0.678052 & 0.538628 & $0.082(11)^{*}$ & $0.273(4)$ \\
H2b & 0.702896 & 0.598291 & 0.774475 & $0.082(11)^{*}$ & $0.727(4)$ \\
\hline
\end{tabular}

Atomic displacement parameters $\left(\AA^{2}\right)$

\begin{tabular}{|c|c|c|c|c|c|c|}
\hline & $U^{11}$ & $U^{22}$ & $U^{33}$ & $U^{12}$ & $U^{13}$ & $U^{23}$ \\
\hline $\mathrm{O} 2 \mathrm{~b}$ & $0.0481(12)$ & $0.0570(16)$ & $0.0296(15)$ & $0.0087(11)$ & $0.0152(10)$ & $0.0091(11)$ \\
\hline $\mathrm{O} 21 \mathrm{~b}$ & $0.045(3)$ & 0.053 & $0.046(4)$ & $0.006(3)$ & $0.006(3)$ & $0.023(3)$ \\
\hline O11b & $0.0369(8)$ & $0.0391(10)$ & $0.0583(12)$ & $0.0136(7)$ & $0.0102(8)$ & $0.0073(9)$ \\
\hline $\mathrm{O} 12 \mathrm{~b}$ & $0.0488(9)$ & $0.0643(12)$ & $0.0488(12)$ & $0.0091(8)$ & $0.0232(8)$ & $-0.0030(9)$ \\
\hline $\mathrm{O} 31 \mathrm{~b}$ & $0.0796(13)$ & $0.0853(16)$ & $0.0753(16)$ & $-0.0256(11)$ & $-0.0175(11)$ & $0.0533(13)$ \\
\hline $\mathrm{O} 32 \mathrm{~b}$ & $0.0728(12)$ & $0.0903(16)$ & $0.0642(14)$ & $0.0483(11)$ & $-0.0074(10)$ & $0.0050(11)$ \\
\hline O51b & $0.0542(10)$ & $0.0650(12)$ & $0.0694(13)$ & $0.0077(9)$ & $0.0350(9)$ & $-0.0110(10)$ \\
\hline O52b & $0.0926(13)$ & $0.0722(14)$ & $0.0455(12)$ & $0.0171(11)$ & $0.0314(10)$ & $0.0241(10)$ \\
\hline N3b & $0.0575(13)$ & $0.0347(13)$ & $0.0432(15)$ & $0.0005(11)$ & $-0.0137(11)$ & $-0.0004(10)$ \\
\hline N5b & $0.0476(11)$ & $0.0400(13)$ & $0.0414(13)$ & $-0.0046(10)$ & $0.0193(10)$ & $-0.0067(10)$ \\
\hline $\mathrm{C} 1 \mathrm{~b}$ & $0.0265(10)$ & $0.0220(11)$ & $0.0303(13)$ & $-0.0029(9)$ & $0.0024(9)$ & $-0.0023(10)$ \\
\hline $\mathrm{C} 2 \mathrm{~b}$ & $0.0337(11)$ & $0.0267(12)$ & $0.0294(14)$ & $-0.0061(10)$ & $0.0050(10)$ & $-0.0023(10)$ \\
\hline $\mathrm{C} 3 \mathrm{~b}$ & $0.0361(11)$ & $0.0233(12)$ & $0.0304(14)$ & $0.0002(9)$ & $-0.0053(10)$ & $0.0025(10)$ \\
\hline $\mathrm{C} 4 \mathrm{~b}$ & $0.0257(10)$ & $0.0240(12)$ & $0.0413(15)$ & $-0.0013(9)$ & $-0.0005(10)$ & $-0.0053(11)$ \\
\hline $\mathrm{C} 5 \mathrm{~b}$ & $0.0295(10)$ & $0.0254(12)$ & $0.0274(13)$ & $-0.0052(9)$ & $0.0073(9)$ & $-0.0017(10)$ \\
\hline C6b & $0.0298(10)$ & $0.0218(12)$ & $0.0290(13)$ & $-0.0028(9)$ & $-0.0003(9)$ & $0.0007(10)$ \\
\hline $\mathrm{C} 11 \mathrm{~b}$ & $0.0297(11)$ & $0.0296(13)$ & $0.0464(16)$ & $-0.0042(10)$ & $0.0070(11)$ & $-0.0061(12)$ \\
\hline N1a & $0.0249(8)$ & $0.0327(11)$ & $0.0305(11)$ & $0.0026(8)$ & $0.0041(8)$ & $0.0000(9)$ \\
\hline N8a & $0.0332(9)$ & $0.0493(12)$ & $0.0291(12)$ & $0.0137(9)$ & $0.0037(8)$ & $0.0056(9)$ \\
\hline $\mathrm{C} 2 \mathrm{a}$ & $0.0243(10)$ & $0.0400(14)$ & $0.0451(15)$ & $0.0063(10)$ & $-0.0002(10)$ & $-0.0020(12)$ \\
\hline $\mathrm{C} 3 \mathrm{a}$ & $0.0374(12)$ & $0.0331(14)$ & $0.0465(16)$ & $0.0071(10)$ & $-0.0044(11)$ & $0.0000(11)$ \\
\hline $\mathrm{C} 4 \mathrm{a}$ & $0.0450(12)$ & $0.0397(15)$ & $0.0370(15)$ & -0.0028 & $-0.0060(11)$ & $0.0087(11)$ \\
\hline $\mathrm{C} 5 \mathrm{a}$ & $0.0368(11)$ & $0.0526(16)$ & $0.0319(14)$ & $-0.0008(11)$ & $0.0079(10)$ & $0.0091(12)$ \\
\hline C6a & $0.0355(11)$ & $0.0411(15)$ & $0.0262(13)$ & $0.0079(10)$ & $0.0032(9)$ & $-0.0057(11)$ \\
\hline $\mathrm{C} 7 \mathrm{a}$ & $0.0283(10)$ & $0.0224(12)$ & $0.0291(13)$ & $-0.0012(9)$ & $0.0025(9)$ & $-0.0030(10)$ \\
\hline $\mathrm{C} 9 \mathrm{a}$ & $0.040(3)$ & $0.041(4)$ & $0.0304(18)$ & $-0.001(2)$ & $-0.001(2)$ & $0.004(3)$ \\
\hline $\mathrm{C} 10 \mathrm{a}$ & $0.049(2)$ & $0.042(2)$ & $0.0270(17)$ & $-0.0043(18)$ & $0.0083(15)$ & $-0.0029(19)$ \\
\hline C11a & $0.0367(12)$ & $0.0426(15)$ & $0.0364(15)$ & $0.0003(11)$ & $0.0161(11)$ & $-0.0056(12)$ \\
\hline $\mathrm{C} 13 \mathrm{a}$ & $0.040(3)$ & $0.041(4)$ & $0.0304(18)$ & -0.001 & $-0.001(2)$ & 0.004 \\
\hline $\mathrm{C} 12 \mathrm{a}$ & $0.049(2)$ & $0.042(2)$ & $0.0270(17)$ & $-0.0043(18)$ & $0.0083(15)$ & $-0.0029(19)$ \\
\hline
\end{tabular}

Geometric parameters $\left(A,{ }^{o}\right)$

\begin{tabular}{llll}
\hline $\mathrm{O} 2 \mathrm{~b}-\mathrm{C} 2 \mathrm{~b}$ & $1.285(3)$ & $\mathrm{C} 2 \mathrm{a}-\mathrm{H} 22 \mathrm{a}$ & 0.99 \\
$\mathrm{O} 2 \mathrm{~b}-\mathrm{H} 2 \mathrm{~b}$ & $1.103(2)$ & $\mathrm{C} 3 \mathrm{a}-\mathrm{C} 4 \mathrm{a}$ & $1.518(3)$ \\
$\mathrm{H} 61 \mathrm{~b}-\mathrm{C} 2 \mathrm{~b}$ & 0.95 & $\mathrm{C} 3 \mathrm{a}-\mathrm{H} 31 \mathrm{a}$ & 0.99 \\
$\mathrm{O} 21 \mathrm{~b}-\mathrm{C} 6 \mathrm{~b}$ & $1.311(6)$ & $\mathrm{C} 3 \mathrm{a}-\mathrm{H} 32 \mathrm{a}$ & 0.99 \\
$\mathrm{O} 21 \mathrm{~b}-\mathrm{H} 21 \mathrm{~b}$ & $1.303(6)$ & $\mathrm{C} 4 \mathrm{a}-\mathrm{C} 5 \mathrm{a}$ & $1.520(3)$
\end{tabular}




\begin{tabular}{|c|c|c|c|}
\hline $\mathrm{H} 6 \mathrm{~b}-\mathrm{C} 6 \mathrm{~b}$ & 0.95 & $\mathrm{C} 4 \mathrm{a}-\mathrm{H} 41 \mathrm{a}$ & 0.99 \\
\hline $\mathrm{O} 11 \mathrm{~b}-\mathrm{C} 11 \mathrm{~b}$ & $1.248(3)$ & $\mathrm{C} 4 \mathrm{a}-\mathrm{H} 42 \mathrm{a}$ & 0.99 \\
\hline $\mathrm{O} 11 \mathrm{~b}-\mathrm{H} 21 \mathrm{~b}$ & $1.1454(16)$ & $\mathrm{C} 5 \mathrm{a}-\mathrm{C} 6 \mathrm{a}$ & $1.530(3)$ \\
\hline $\mathrm{O} 12 \mathrm{~b}-\mathrm{C} 11 \mathrm{~b}$ & $1.272(3)$ & $\mathrm{C} 5 \mathrm{a}-\mathrm{H} 51 \mathrm{a}$ & 0.99 \\
\hline $\mathrm{O} 12 \mathrm{~b}-\mathrm{H} 2 \mathrm{~b}$ & $1.3846(15)$ & $\mathrm{C} 5 \mathrm{a}-\mathrm{H} 52 \mathrm{a}$ & 0.99 \\
\hline $\mathrm{O} 31 \mathrm{~b}-\mathrm{N} 3 \mathrm{~b}$ & $1.205(3)$ & $\mathrm{C} 6 \mathrm{a}-\mathrm{C} 7 \mathrm{a}$ & $1.490(3)$ \\
\hline $\mathrm{O} 32 \mathrm{~b}-\mathrm{N} 3 \mathrm{~b}$ & $1.230(3)$ & $\mathrm{C} 6 \mathrm{a}-\mathrm{H} 61 \mathrm{a}$ & 0.99 \\
\hline $\mathrm{O} 51 \mathrm{~b}-\mathrm{N} 5 \mathrm{~b}$ & $1.219(3)$ & $\mathrm{C} 6 \mathrm{a}-\mathrm{H} 62 \mathrm{a}$ & 0.99 \\
\hline $\mathrm{O} 52 \mathrm{~b}-\mathrm{N} 5 \mathrm{~b}$ & $1.230(3)$ & $\mathrm{C} 9 \mathrm{a}-\mathrm{C} 10 \mathrm{a}$ & $1.508(6)$ \\
\hline $\mathrm{N} 3 \mathrm{~b}-\mathrm{C} 3 \mathrm{~b}$ & $1.466(3)$ & $\mathrm{C} 9 \mathrm{a}-\mathrm{C} 13 \mathrm{a}$ & $0.581(13)$ \\
\hline $\mathrm{N} 5 \mathrm{~b}-\mathrm{C} 5 \mathrm{~b}$ & $1.456(3)$ & $\mathrm{C} 9 \mathrm{a}-\mathrm{C} 12 \mathrm{a}$ & $1.323(10)$ \\
\hline $\mathrm{C} 1 \mathrm{~b}-\mathrm{C} 2 \mathrm{~b}$ & $1.407(3)$ & $\mathrm{C} 9 \mathrm{a}-\mathrm{H} 91 \mathrm{a}$ & 0.99 \\
\hline $\mathrm{C} 1 \mathrm{~b}-\mathrm{C} 6 \mathrm{~b}$ & $1.387(3)$ & $\mathrm{C} 9 \mathrm{a}-\mathrm{H} 92 \mathrm{a}$ & 0.99 \\
\hline $\mathrm{C} 1 \mathrm{~b}-\mathrm{C} 11 \mathrm{~b}$ & $1.497(3)$ & $\mathrm{C} 9 \mathrm{a}-\mathrm{H} 16 \mathrm{a}$ & 0.9922 \\
\hline $\mathrm{C} 2 \mathrm{~b}-\mathrm{C} 3 \mathrm{~b}$ & $1.404(3)$ & $\mathrm{C} 10 \mathrm{a}-\mathrm{C} 11 \mathrm{a}$ & $1.501(4)$ \\
\hline $\mathrm{C} 3 \mathrm{~b}-\mathrm{C} 4 \mathrm{~b}$ & $1.371(3)$ & $\mathrm{C} 10 \mathrm{a}-\mathrm{C} 13 \mathrm{a}$ & $1.358(14)$ \\
\hline$C 4 b-C 5 b$ & 1.375 & $\mathrm{C} 10 \mathrm{a}-\mathrm{H} 10 \mathrm{a}$ & 0.99 \\
\hline $\mathrm{C} 4 \mathrm{~b}-\mathrm{H} 4 \mathrm{~b}$ & 0.95 & $\mathrm{C} 10 \mathrm{a}-\mathrm{H} 11 \mathrm{a}$ & 0.99 \\
\hline $\mathrm{C} 5 \mathrm{~b}-\mathrm{C} 6 \mathrm{~b}$ & $1.392(3)$ & $\mathrm{C} 10 \mathrm{a}-\mathrm{H} 15 \mathrm{a}$ & 1.207 \\
\hline $\mathrm{N} 1 \mathrm{a}-\mathrm{C} 2 \mathrm{a}$ & $1.471(2)$ & $\mathrm{C} 11 \mathrm{a}-\mathrm{C} 12 \mathrm{a}$ & $1.530(8)$ \\
\hline $\mathrm{N} 1 \mathrm{a}-\mathrm{C} 7 \mathrm{a}$ & $1.313(2)$ & $\mathrm{C} 11 \mathrm{a}-\mathrm{H} 12 \mathrm{a}$ & $0.977(15)$ \\
\hline $\mathrm{N} 1 \mathrm{a}-\mathrm{C} 11 \mathrm{a}$ & $1.470(3)$ & $\mathrm{C} 11 \mathrm{a}-\mathrm{H} 13 \mathrm{a}$ & $0.978(13)$ \\
\hline $\mathrm{N} 8 \mathrm{a}-\mathrm{C} 7 \mathrm{a}$ & $1.311(2)$ & $\mathrm{C} 13 \mathrm{a}-\mathrm{C} 12 \mathrm{a}$ & $1.534(16)$ \\
\hline $\mathrm{N} 8 \mathrm{a}-\mathrm{C} 9 \mathrm{a}$ & $1.467(6)$ & $\mathrm{C} 13 \mathrm{a}-\mathrm{H} 92 \mathrm{a}$ & 0.9083 \\
\hline $\mathrm{N} 8 \mathrm{a}-\mathrm{C} 13 \mathrm{a}$ & $1.498(15)$ & $\mathrm{C} 13 \mathrm{a}-\mathrm{H} 16 \mathrm{a}$ & 0.99 \\
\hline $\mathrm{N} 8 \mathrm{a}-\mathrm{H} 8 \mathrm{a}$ & $0.9604(17)$ & $\mathrm{C} 13 \mathrm{a}-\mathrm{H} 17 \mathrm{a}$ & 0.99 \\
\hline $\mathrm{C} 2 \mathrm{a}-\mathrm{C} 3 \mathrm{a}$ & $1.514(3)$ & $\mathrm{C} 12 \mathrm{a}-\mathrm{H} 14 \mathrm{a}$ & 0.99 \\
\hline $\mathrm{C} 2 \mathrm{a}-\mathrm{H} 21 \mathrm{a}$ & 0.99 & $\mathrm{C} 12 \mathrm{a}-\mathrm{H} 15 \mathrm{a}$ & 0.99 \\
\hline $\mathrm{H} 6 \mathrm{~b}-\mathrm{O} 21 \mathrm{~b}-\mathrm{H} 21 \mathrm{~b}$ & 102.88 & $\mathrm{~N} 8 \mathrm{a}-\mathrm{C} 9 \mathrm{a}-\mathrm{C} 13 \mathrm{a}$ & $81.6(16)$ \\
\hline $\mathrm{C} 11 \mathrm{~b}-\mathrm{O} 12 \mathrm{~b}-\mathrm{H} 2 \mathrm{~b}$ & $98.61(14)$ & $\mathrm{N} 8 \mathrm{a}-\mathrm{C} 9 \mathrm{a}-\mathrm{C} 12 \mathrm{a}$ & $118.3(5)$ \\
\hline $\mathrm{O} 31 \mathrm{~b}-\mathrm{N} 3 \mathrm{~b}-\mathrm{O} 32 \mathrm{~b}$ & $124.0(2)$ & $\mathrm{N} 8 \mathrm{a}-\mathrm{C} 9 \mathrm{a}-\mathrm{H} 91 \mathrm{a}$ & 109.47 \\
\hline $\mathrm{O} 31 \mathrm{~b}-\mathrm{N} 3 \mathrm{~b}-\mathrm{C} 3 \mathrm{~b}$ & $118.6(2)$ & $\mathrm{N} 8 \mathrm{a}-\mathrm{C} 9 \mathrm{a}-\mathrm{H} 92 \mathrm{a}$ & 109.47 \\
\hline $\mathrm{O} 32 \mathrm{~b}-\mathrm{N} 3 \mathrm{~b}-\mathrm{C} 3 \mathrm{~b}$ & $117.4(2)$ & $\mathrm{N} 8 \mathrm{a}-\mathrm{C} 9 \mathrm{a}-\mathrm{H} 14 \mathrm{a}$ & 114.78 \\
\hline $\mathrm{O} 51 \mathrm{~b}-\mathrm{N} 5 \mathrm{~b}-\mathrm{O} 52 \mathrm{~b}$ & $123.6(2)$ & $\mathrm{N} 8 \mathrm{a}-\mathrm{C} 9 \mathrm{a}-\mathrm{H} 16 \mathrm{a}$ & 111.65 \\
\hline $\mathrm{O} 51 \mathrm{~b}-\mathrm{N} 5 \mathrm{~b}-\mathrm{C} 5 \mathrm{~b}$ & $118.57(18)$ & $\mathrm{C} 10 \mathrm{a}-\mathrm{C} 9 \mathrm{a}-\mathrm{H} 91 \mathrm{a}$ & 109.47 \\
\hline $\mathrm{O} 52 \mathrm{~b}-\mathrm{N} 5 \mathrm{~b}-\mathrm{C} 5 \mathrm{~b}$ & 117.83 & $\mathrm{C} 10 \mathrm{a}-\mathrm{C} 9 \mathrm{a}-\mathrm{H} 92 \mathrm{a}$ & 109.47 \\
\hline $\mathrm{C} 2 \mathrm{~b}-\mathrm{C} 1 \mathrm{~b}-\mathrm{C} 6 \mathrm{~b}$ & $120.82(17)$ & $\mathrm{C} 13 \mathrm{a}-\mathrm{C} 9 \mathrm{a}-\mathrm{C} 12 \mathrm{a}$ & $99.9(15)$ \\
\hline $\mathrm{C} 2 \mathrm{~b}-\mathrm{C} 1 \mathrm{~b}-\mathrm{C} 11 \mathrm{~b}$ & $119.6(2)$ & $\mathrm{C} 13 \mathrm{a}-\mathrm{C} 9 \mathrm{a}-\mathrm{H} 91 \mathrm{a}$ & 168.74 \\
\hline $\mathrm{C} 6 \mathrm{~b}-\mathrm{C} 1 \mathrm{~b}-\mathrm{C} 11 \mathrm{~b}$ & $119.51(18)$ & $\mathrm{C} 13 \mathrm{a}-\mathrm{C} 9 \mathrm{a}-\mathrm{H} 14 \mathrm{a}$ & 137.56 \\
\hline $\mathrm{O} 2 \mathrm{~b}-\mathrm{C} 2 \mathrm{~b}-\mathrm{C} 1 \mathrm{~b}$ & $121.84(19)$ & $\mathrm{C} 12 \mathrm{a}-\mathrm{C} 9 \mathrm{a}-\mathrm{H} 92 \mathrm{a}$ & 126.91 \\
\hline $\mathrm{O} 2 \mathrm{~b}-\mathrm{C} 2 \mathrm{~b}-\mathrm{C} 3 \mathrm{~b}$ & $120.77(19)$ & $\mathrm{C} 12 \mathrm{a}-\mathrm{C} 9 \mathrm{a}-\mathrm{H} 16 \mathrm{a}$ & 127.82 \\
\hline $\mathrm{H} 61 \mathrm{~b}-\mathrm{C} 2 \mathrm{~b}-\mathrm{C} 1 \mathrm{~b}$ & 121.37 & $\mathrm{H} 91 \mathrm{a}-\mathrm{C} 9 \mathrm{a}-\mathrm{H} 92 \mathrm{a}$ & 111.59 \\
\hline $\mathrm{H} 61 \mathrm{~b}-\mathrm{C} 2 \mathrm{~b}-\mathrm{C} 3 \mathrm{~b}$ & 121.37 & $\mathrm{H} 14 \mathrm{a}-\mathrm{C} 9 \mathrm{a}-\mathrm{H} 16 \mathrm{a}$ & 126.91 \\
\hline $\mathrm{C} 1 \mathrm{~b}-\mathrm{C} 2 \mathrm{~b}-\mathrm{C} 3 \mathrm{~b}$ & $117.3(2)$ & $\mathrm{H} 14 \mathrm{a}-\mathrm{C} 9 \mathrm{a}-\mathrm{H} 17 \mathrm{a}$ & 129.78 \\
\hline $\mathrm{N} 3 b-\mathrm{C} 3 \mathrm{~b}-\mathrm{C} 2 \mathrm{~b}$ & $120.4(2)$ & $\mathrm{H} 16 \mathrm{a}-\mathrm{C} 9 \mathrm{a}-\mathrm{H} 17 \mathrm{a}$ & 77.55 \\
\hline $\mathrm{N} 3 \mathrm{~b}-\mathrm{C} 3 \mathrm{~b}-\mathrm{C} 4 \mathrm{~b}$ & $117.12(18)$ & $\mathrm{C} 9 \mathrm{a}-\mathrm{C} 10 \mathrm{a}-\mathrm{C} 11 \mathrm{a}$ & $109.8(3)$ \\
\hline $\mathrm{C} 2 \mathrm{~b}-\mathrm{C} 3 \mathrm{~b}-\mathrm{C} 4 \mathrm{~b}$ & $122.44(18)$ & $\mathrm{C} 11 \mathrm{a}-\mathrm{C} 10 \mathrm{a}-\mathrm{C} 13 \mathrm{a}$ & $122.2(6)$ \\
\hline
\end{tabular}




\begin{tabular}{|c|c|}
\hline $\mathrm{C} 3 \mathrm{~b}-\mathrm{C} 4 \mathrm{~b}-\mathrm{C} 5 \mathrm{~b}$ & $118.75(18)$ \\
\hline $\mathrm{C} 3 \mathrm{~b}-\mathrm{C} 4 \mathrm{~b}-\mathrm{H} 4 \mathrm{~b}$ & 120.62 \\
\hline $\mathrm{C} 5 \mathrm{~b}-\mathrm{C} 4 \mathrm{~b}-\mathrm{H} 4 \mathrm{~b}$ & 120.62 \\
\hline $\mathrm{N} 5 \mathrm{~b}-\mathrm{C} 5 \mathrm{~b}-\mathrm{C} 4 \mathrm{~b}$ & $118.74(17)$ \\
\hline $\mathrm{N} 5 \mathrm{~b}-\mathrm{C} 5 \mathrm{~b}-\mathrm{C} 6 \mathrm{~b}$ & $119.80(17)$ \\
\hline$C 4 b-C 5 b-C 6 b$ & $121.5(2)$ \\
\hline $\mathrm{O} 21 \mathrm{~b}-\mathrm{C} 6 \mathrm{~b}-\mathrm{C} 1 \mathrm{~b}$ & $118.4(3)$ \\
\hline $\mathrm{O} 21 \mathrm{~b}-\mathrm{C} 6 \mathrm{~b}-\mathrm{C} 5 \mathrm{~b}$ & $122.4(3)$ \\
\hline $\mathrm{H} 6 \mathrm{~b}-\mathrm{C} 6 \mathrm{~b}-\mathrm{C} 1 \mathrm{~b}$ & 120.41 \\
\hline $\mathrm{H} 6 \mathrm{~b}-\mathrm{C} 6 \mathrm{~b}-\mathrm{C} 5 \mathrm{~b}$ & 120.41 \\
\hline $\mathrm{C} 1 \mathrm{~b}-\mathrm{C} 6 \mathrm{~b}-\mathrm{C} 5 \mathrm{~b}$ & $119.17(18)$ \\
\hline $\mathrm{O} 11 \mathrm{~b}-\mathrm{C} 11 \mathrm{~b}-\mathrm{O} 12 \mathrm{~b}$ & $123.9(2)$ \\
\hline $\mathrm{O} 11 \mathrm{~b}-\mathrm{C} 11 \mathrm{~b}-\mathrm{C} 1 \mathrm{~b}$ & $119.4(2)$ \\
\hline $\mathrm{O} 12 \mathrm{~b}-\mathrm{C} 11 \mathrm{~b}-\mathrm{C} 1 \mathrm{~b}$ & $116.70(19)$ \\
\hline $\mathrm{C} 2 \mathrm{a}-\mathrm{N} 1 \mathrm{a}-\mathrm{C} 7 \mathrm{a}$ & $121.83(17)$ \\
\hline $\mathrm{C} 2 \mathrm{a}-\mathrm{N} 1 \mathrm{a}-\mathrm{C} 11 \mathrm{a}$ & $116.29(16)$ \\
\hline $\mathrm{C} 7 \mathrm{a}-\mathrm{N} 1 \mathrm{a}-\mathrm{C} 11 \mathrm{a}$ & $121.87(16)$ \\
\hline $\mathrm{C} 7 \mathrm{a}-\mathrm{N} 8 \mathrm{a}-\mathrm{C} 9 \mathrm{a}$ & $121.9(2)$ \\
\hline $\mathrm{C} 7 \mathrm{a}-\mathrm{N} 8 \mathrm{a}-\mathrm{C} 13 \mathrm{a}$ & $122.3(5)$ \\
\hline $\mathrm{C} 7 \mathrm{a}-\mathrm{N} 8 \mathrm{a}-\mathrm{H} 8 \mathrm{a}$ & $119.53(19)$ \\
\hline $\mathrm{C} 9 \mathrm{a}-\mathrm{N} 8 \mathrm{a}-\mathrm{C} 13 \mathrm{a}$ & $22.6(5)$ \\
\hline $\mathrm{C} 9 \mathrm{a}-\mathrm{N} 8 \mathrm{a}-\mathrm{H} 8 \mathrm{a}$ & $118.5(2)$ \\
\hline $\mathrm{C} 13 \mathrm{a}-\mathrm{N} 8 \mathrm{a}-\mathrm{H} 8 \mathrm{a}$ & $114.3(5)$ \\
\hline $\mathrm{N} 1 \mathrm{a}-\mathrm{C} 2 \mathrm{a}-\mathrm{C} 3 \mathrm{a}$ & $114.01(15)$ \\
\hline $\mathrm{N} 1 \mathrm{a}-\mathrm{C} 2 \mathrm{a}-\mathrm{H} 21 \mathrm{a}$ & 109.47 \\
\hline $\mathrm{N} 1 \mathrm{a}-\mathrm{C} 2 \mathrm{a}-\mathrm{H} 22 \mathrm{a}$ & 109.47 \\
\hline $\mathrm{C} 3 \mathrm{a}-\mathrm{C} 2 \mathrm{a}-\mathrm{H} 21 \mathrm{a}$ & 109.47 \\
\hline $\mathrm{C} 3 \mathrm{a}-\mathrm{C} 2 \mathrm{a}-\mathrm{H} 22 \mathrm{a}$ & 109.47 \\
\hline $\mathrm{H} 21 \mathrm{a}-\mathrm{C} 2 \mathrm{a}-\mathrm{H} 22 \mathrm{a}$ & 104.52 \\
\hline $\mathrm{C} 2 \mathrm{a}-\mathrm{C} 3 \mathrm{a}-\mathrm{C} 4 \mathrm{a}$ & $114.39(18)$ \\
\hline $\mathrm{C} 2 \mathrm{a}-\mathrm{C} 3 \mathrm{a}-\mathrm{H} 31 \mathrm{a}$ & 109.47 \\
\hline $\mathrm{C} 2 \mathrm{a}-\mathrm{C} 3 \mathrm{a}-\mathrm{H} 32 \mathrm{a}$ & 109.47 \\
\hline $\mathrm{C} 4 \mathrm{a}-\mathrm{C} 3 \mathrm{a}-\mathrm{H} 31 \mathrm{a}$ & 109.47 \\
\hline $\mathrm{C} 4 \mathrm{a}-\mathrm{C} 3 \mathrm{a}-\mathrm{H} 32 \mathrm{a}$ & 109.47 \\
\hline $\mathrm{H} 31 \mathrm{a}-\mathrm{C} 3 \mathrm{a}-\mathrm{H} 32 \mathrm{a}$ & 104.05 \\
\hline $\mathrm{C} 3 \mathrm{a}-\mathrm{C} 4 \mathrm{a}-\mathrm{C} 5 \mathrm{a}$ & $114.58(18)$ \\
\hline $\mathrm{C} 3 \mathrm{a}-\mathrm{C} 4 \mathrm{a}-\mathrm{H} 41 \mathrm{a}$ & 109.47 \\
\hline $\mathrm{C} 3 \mathrm{a}-\mathrm{C} 4 \mathrm{a}-\mathrm{H} 42 \mathrm{a}$ & 109.47 \\
\hline $\mathrm{C} 5 \mathrm{a}-\mathrm{C} 4 \mathrm{a}-\mathrm{H} 41 \mathrm{a}$ & 109.47 \\
\hline $\mathrm{C} 5 \mathrm{a}-\mathrm{C} 4 \mathrm{a}-\mathrm{H} 42 \mathrm{a}$ & 109.47 \\
\hline $\mathrm{H} 41 \mathrm{a}-\mathrm{C} 4 \mathrm{a}-\mathrm{H} 42 \mathrm{a}$ & 103.83 \\
\hline $\mathrm{C} 4 \mathrm{a}-\mathrm{C} 5 \mathrm{a}-\mathrm{C} 6 \mathrm{a}$ & $114.44(16)$ \\
\hline $\mathrm{C} 4 \mathrm{a}-\mathrm{C} 5 \mathrm{a}-\mathrm{H} 51 \mathrm{a}$ & 109.47 \\
\hline $\mathrm{C} 4 \mathrm{a}-\mathrm{C} 5 \mathrm{a}-\mathrm{H} 52 \mathrm{a}$ & 109.47 \\
\hline $\mathrm{C} 6 \mathrm{a}-\mathrm{C} 5 \mathrm{a}-\mathrm{H} 51 \mathrm{a}$ & 109.47 \\
\hline $\mathrm{C} 6 \mathrm{a}-\mathrm{C} 5 \mathrm{a}-\mathrm{H} 52 \mathrm{a}$ & 109.47 \\
\hline $\mathrm{H} 51 \mathrm{a}-\mathrm{C} 5 \mathrm{a}-\mathrm{H} 52 \mathrm{a}$ & 104 \\
\hline $\mathrm{C} 5 \mathrm{a}-\mathrm{C} 6 \mathrm{a}-\mathrm{C} 7 \mathrm{a}$ & $112.99(17)$ \\
\hline
\end{tabular}

\begin{tabular}{|c|c|}
\hline $\mathrm{C} 11 \mathrm{a}-\mathrm{C} 10 \mathrm{a}-\mathrm{H} 10 \mathrm{a}$ & 109.47 \\
\hline $\mathrm{C} 11 \mathrm{a}-\mathrm{C} 10 \mathrm{a}-\mathrm{H} 11 \mathrm{a}$ & 109.47 \\
\hline $\mathrm{C} 11 \mathrm{a}-\mathrm{C} 10 \mathrm{a}-\mathrm{H} 17 \mathrm{a}$ & 132.02 \\
\hline $\mathrm{C} 13 \mathrm{a}-\mathrm{C} 10 \mathrm{a}-\mathrm{H} 11 \mathrm{a}$ & 116.43 \\
\hline $\mathrm{C} 13 \mathrm{a}-\mathrm{C} 10 \mathrm{a}-\mathrm{H} 15 \mathrm{a}$ & 108.54 \\
\hline $\mathrm{C} 12 \mathrm{a}-\mathrm{C} 10 \mathrm{a}-\mathrm{H} 17 \mathrm{a}$ & 124.07 \\
\hline $\mathrm{H} 10 \mathrm{a}-\mathrm{C} 10 \mathrm{a}-\mathrm{H} 11 \mathrm{a}$ & 109.16 \\
\hline $\mathrm{H} 10 \mathrm{a}-\mathrm{C} 10 \mathrm{a}-\mathrm{H} 15 \mathrm{a}$ & 132.06 \\
\hline $\mathrm{H} 11 \mathrm{a}-\mathrm{C} 10 \mathrm{a}-\mathrm{H} 17 \mathrm{a}$ & 117.48 \\
\hline $\mathrm{H} 15 \mathrm{a}-\mathrm{C} 10 \mathrm{a}-\mathrm{H} 17 \mathrm{a}$ & 127.34 \\
\hline $\mathrm{N} 1 \mathrm{a}-\mathrm{C} 11 \mathrm{a}-\mathrm{C} 10 \mathrm{a}$ & $111.6(2)$ \\
\hline $\mathrm{N} 1 \mathrm{a}-\mathrm{C} 11 \mathrm{a}-\mathrm{C} 12 \mathrm{a}$ & $112.2(4)$ \\
\hline $\mathrm{N} 1 \mathrm{a}-\mathrm{C} 11 \mathrm{a}-\mathrm{H} 12 \mathrm{a}$ & $108.1(11)$ \\
\hline $\mathrm{N} 1 \mathrm{a}-\mathrm{C} 11 \mathrm{a}-\mathrm{H} 13 \mathrm{a}$ & $107.3(12)$ \\
\hline $\mathrm{C} 10 \mathrm{a}-\mathrm{C} 11 \mathrm{a}-\mathrm{H} 12 \mathrm{a}$ & $120.7(10)$ \\
\hline $\mathrm{C} 10 \mathrm{a}-\mathrm{C} 11 \mathrm{a}-\mathrm{H} 13 \mathrm{a}$ & $105.2(10)$ \\
\hline $\mathrm{C} 12 \mathrm{a}-\mathrm{C} 11 \mathrm{a}-\mathrm{H} 13 \mathrm{a}$ & $131.8(11)$ \\
\hline $\mathrm{H} 12 \mathrm{a}-\mathrm{C} 11 \mathrm{a}-\mathrm{H} 13 \mathrm{a}$ & $102.9(15)$ \\
\hline $\mathrm{N} 8 \mathrm{a}-\mathrm{C} 13 \mathrm{a}-\mathrm{C} 9 \mathrm{a}$ & $75.8(15)$ \\
\hline $\mathrm{N} 8 \mathrm{a}-\mathrm{C} 13 \mathrm{a}-\mathrm{C} 10 \mathrm{a}$ & $114.0(9)$ \\
\hline $\mathrm{N} 8 \mathrm{a}-\mathrm{C} 13 \mathrm{a}-\mathrm{C} 12 \mathrm{a}$ & $104.5(8)$ \\
\hline $\mathrm{N} 8 \mathrm{a}-\mathrm{C} 13 \mathrm{a}-\mathrm{H} 10 \mathrm{a}$ & 113.12 \\
\hline $\mathrm{N} 8 \mathrm{a}-\mathrm{C} 13 \mathrm{a}-\mathrm{H} 92 \mathrm{a}$ & 112.32 \\
\hline $\mathrm{N} 8 \mathrm{a}-\mathrm{C} 13 \mathrm{a}-\mathrm{H} 16 \mathrm{a}$ & 109.47 \\
\hline $\mathrm{N} 8 \mathrm{a}-\mathrm{C} 13 \mathrm{a}-\mathrm{H} 17 \mathrm{a}$ & 109.47 \\
\hline $\mathrm{C} 9 \mathrm{a}-\mathrm{C} 13 \mathrm{a}-\mathrm{H} 10 \mathrm{a}$ & 130.08 \\
\hline $\mathrm{C} 9 \mathrm{a}-\mathrm{C} 13 \mathrm{a}-\mathrm{H} 17 \mathrm{a}$ & 167.66 \\
\hline $\mathrm{C} 10 \mathrm{a}-\mathrm{C} 13 \mathrm{a}-\mathrm{H} 92 \mathrm{a}$ & 129.79 \\
\hline $\mathrm{C} 10 \mathrm{a}-\mathrm{C} 13 \mathrm{a}-\mathrm{H} 16 \mathrm{a}$ & 129.35 \\
\hline $\mathrm{C} 12 \mathrm{a}-\mathrm{C} 13 \mathrm{a}-\mathrm{H} 92 \mathrm{a}$ & 113.84 \\
\hline $\mathrm{C} 12 \mathrm{a}-\mathrm{C} 13 \mathrm{a}-\mathrm{H} 16 \mathrm{a}$ & 109.47 \\
\hline $\mathrm{C} 12 \mathrm{a}-\mathrm{C} 13 \mathrm{a}-\mathrm{H} 17 \mathrm{a}$ & 109.47 \\
\hline $\mathrm{H} 10 \mathrm{a}-\mathrm{C} 13 \mathrm{a}-\mathrm{H} 91 \mathrm{a}$ & 126.53 \\
\hline $\mathrm{H} 10 \mathrm{a}-\mathrm{C} 13 \mathrm{a}-\mathrm{H} 92 \mathrm{a}$ & 130.35 \\
\hline $\mathrm{H} 10 \mathrm{a}-\mathrm{C} 13 \mathrm{a}-\mathrm{H} 16 \mathrm{a}$ & 135.32 \\
\hline $\mathrm{H} 92 \mathrm{a}-\mathrm{C} 13 \mathrm{a}-\mathrm{H} 17 \mathrm{a}$ & 107.21 \\
\hline $\mathrm{H} 16 \mathrm{a}-\mathrm{C} 13 \mathrm{a}-\mathrm{H} 17 \mathrm{a}$ & 114.05 \\
\hline $\mathrm{C} 9 \mathrm{a}-\mathrm{C} 12 \mathrm{a}-\mathrm{C} 11 \mathrm{a}$ & $119.1(6)$ \\
\hline $\mathrm{C} 9 \mathrm{a}-\mathrm{C} 12 \mathrm{a}-\mathrm{H} 11 \mathrm{a}$ & 111.16 \\
\hline $\mathrm{C} 9 \mathrm{a}-\mathrm{C} 12 \mathrm{a}-\mathrm{H} 15 \mathrm{a}$ & 119.13 \\
\hline $\mathrm{C} 10 \mathrm{a}-\mathrm{C} 12 \mathrm{a}-\mathrm{H} 91 \mathrm{a}$ & 125.59 \\
\hline $\mathrm{C} 10 \mathrm{a}-\mathrm{C} 12 \mathrm{a}-\mathrm{H} 14 \mathrm{a}$ & 169.66 \\
\hline $\mathrm{C} 11 \mathrm{a}-\mathrm{C} 12 \mathrm{a}-\mathrm{C} 13 \mathrm{a}$ & $109.6(7)$ \\
\hline $\mathrm{C} 11 \mathrm{a}-\mathrm{C} 12 \mathrm{a}-\mathrm{H} 91 \mathrm{a}$ & 130.75 \\
\hline $\mathrm{C} 11 \mathrm{a}-\mathrm{C} 12 \mathrm{a}-\mathrm{H} 14 \mathrm{a}$ & 109.47 \\
\hline $\mathrm{C} 11 \mathrm{a}-\mathrm{C} 12 \mathrm{a}-\mathrm{H} 15 \mathrm{a}$ & 109.47 \\
\hline $\mathrm{C} 13 \mathrm{a}-\mathrm{C} 12 \mathrm{a}-\mathrm{H} 14 \mathrm{a}$ & 109.47 \\
\hline $\mathrm{C} 13 \mathrm{a}-\mathrm{C} 12 \mathrm{a}-\mathrm{H} 15 \mathrm{a}$ & 109.47 \\
\hline
\end{tabular}




$\begin{array}{llll}\text { C5a-C6a-H61a } & 109.47 & \text { H91a-C12a-H11a } & 130.35 \\ \text { C5a-C6a-H62a } & 109.47 & \text { H91a-C12a-H15a } & 118.71 \\ \text { C7a-C6a-H61a } & 109.47 & \text { H11a-C12a-H14a } & 133.54 \\ \text { C7a-C6a-H62a } & 109.47 & \text { H14a-C12a-H15a } & 109.31 \\ \text { H61a-C6a-H62a } & 105.71 & \text { O21b-H21b-O11b } & 167.3(3) \\ \text { N1a-C7a-N8a } & 121.93(19) & \text { H6b-H21b-O11b } & 152.98 \\ \text { N1a-C7a-C6a } & 120.45(16) & \text { O2b-H2b-O12b } & 166.81(13) \\ \text { N8a-C7a-C6a } & 117.59(17) & \text { H61b-H2b-O12b } & 150.18 \\ \text { N8a-C9a-C10a } & 107.3(3) & & \end{array}$

Hydrogen-bond geometry $\left(\AA,{ }^{\circ}\right)$

\begin{tabular}{lllll}
\hline$D-\mathrm{H} \cdots A$ & $D-\mathrm{H}$ & $\mathrm{H} \cdots A$ & $D \cdots A$ & $D-\mathrm{H} \cdots A$ \\
\hline $\mathrm{N} 8 a-\mathrm{H} 8 a \cdots \mathrm{O} 11 b$ & $0.9604(17)$ & $1.9329(15)$ & $2.864(2)$ & $162.83(11)$ \\
$\mathrm{C} 10 a-\mathrm{H} 10 a \cdots \mathrm{O} 32 b^{\mathrm{i}}$ & 0.99 & 2.44 & $3.248(4)$ & 138 \\
$\mathrm{C} 2 a-\mathrm{H} 21 a \cdots \mathrm{O} 31 b^{\mathrm{ii}}$ & 0.99 & 2.48 & $3.275(3)$ & 137 \\
$\mathrm{C} 6 a-\mathrm{H} 62 a \cdots \mathrm{O} 21 b^{\mathrm{iii}}$ & 0.99 & 2.44 & $3.152(6)$ & 128 \\
$\mathrm{C} 10 a-\mathrm{H} 11 a \cdots \mathrm{O} 21 b^{\mathrm{iv}}$ & 0.99 & 2.47 & $3.033(7)$ & 116 \\
$\mathrm{O} 21 b-\mathrm{H} 21 b \cdots \mathrm{O} 11 b$ & $1.303(6)$ & $1.1454(16)$ & $2.433(6)$ & $167.3(3)$ \\
$\mathrm{O} 11 b-\mathrm{H} 21 b \cdots \mathrm{O} 21 b$ & $1.1454(16)$ & $1.303(6)$ & $2.433(6)$ & $167.3(3)$ \\
$\mathrm{O} 12 b-\mathrm{H} 2 b \cdots \mathrm{O} 2 b$ & $1.3846(15)$ & $1.103(2)$ & $2.471(2)$ & $166.81(13)$ \\
\hline
\end{tabular}

Symmetry codes: (i) $-x+3 / 2, y+1 / 2,-z+3 / 2$; (ii) $-x+1 / 2, y+1 / 2,-z+3 / 2$; (iii) $x-1, y, z$; (iv) $x-1 / 2,-y+3 / 2, z+1 / 2$.

4-(Diphenylmethyl)-1-(3-phenylprop-2-en-1-yl)piperazin-1-ium 2-carboxy-4,6-dinitrophenolate (YAXPOE)

Crystal data

$\mathrm{C}_{26} \mathrm{H}_{29} \mathrm{~N}_{2}^{+} \cdot \mathrm{C}_{7} \mathrm{H}_{3} \mathrm{~N}_{2} \mathrm{O}_{7}^{-}$

$M_{r}=596.63$

Monoclinic, $P 2_{1} / c$

Hall symbol: -P 2ybc

$a=14.5648$ (3) $\AA$

$b=12.9374(3) \AA$

$c=16.1619(3) \AA$

$\beta=103.900(1)^{\circ}$

$V=2956.22(11) \AA^{3}$

$Z=4$

\section{Data collection}

\section{Bruker APEXII CCD}

diffractometer

Radiation source: fine-focus sealed tube

Graphite monochromator

$\varphi$ and $\omega$ scans

Absorption correction: multi-scan

(SADABS; Bruker, 2008)

$T_{\min }=0.932, T_{\max }=1.000$
$F(000)=1256$

$D_{\mathrm{x}}=1.341 \mathrm{Mg} \mathrm{m}^{-3}$

Melting point: $383 \mathrm{~K}$

Mo $K \alpha$ radiation, $\lambda=0.71073 \AA$

Cell parameters from 9909 reflections

$\theta=2.3-28.3^{\circ}$

$\mu=0.10 \mathrm{~mm}^{-1}$

$T=200 \mathrm{~K}$

Block, yellow

$0.51 \times 0.26 \times 0.17 \mathrm{~mm}$

29552 measured reflections

7344 independent reflections

5724 reflections with $I>3 \sigma(I)$

$R_{\text {int }}=0.015$

$\theta_{\text {max }}=28.3^{\circ}, \theta_{\min }=2.0^{\circ}$

$h=-19 \rightarrow 19$

$k=-17 \rightarrow 17$

$l=-21 \rightarrow 15$ 


\section{Refinement}

Refinement on $F^{2}$

Least-squares matrix: full

$R[F>3 \sigma(F)]=0.054$

$w R(F)=0.190$

$S=1.80$

7344 reflections

399 parameters

0 restraints

120 constraints

Primary atom site location: structure-invariant direct methods

Special details

Refinement. Number of fixed parameters 6
Secondary atom site location: difference Fourier map

Hydrogen site location: difference Fourier map

$\mathrm{H}$ atoms treated by a mixture of independent and constrained refinement

Weighting scheme based on measured s.u.'s $w=$ $1 /\left(\sigma^{2}(I)+0.0063999998 I^{2}\right)$

$(\Delta / \sigma)_{\max }=0.044$

$\Delta \rho_{\max }=0.63 \mathrm{e} \AA^{-3}$

$\Delta \rho_{\min }=-0.28$ e $\AA^{-3}$

Fractional atomic coordinates and isotropic or equivalent isotropic displacement parameters $\left(\AA^{2}\right)$

\begin{tabular}{|c|c|c|c|c|}
\hline & $x$ & $y$ & $z$ & $U_{\text {iso }} * / U_{\text {eq }}$ \\
\hline N1 & $0.25627(8)$ & $0.71181(9)$ & $0.31343(7)$ & 0.0246 \\
\hline H71 & 0.30128 & 0.669474 & 0.349979 & $0.035(4)^{*}$ \\
\hline N2 & $0.10023(8)$ & $0.56683(9)$ & $0.26575(7)$ & 0.0233 \\
\hline $\mathrm{C} 1$ & $0.29865(11)$ & $0.95151(12)$ & $0.41661(10)$ & $0.0334(5)$ \\
\hline H1a & 0.245898 & 0.980932 & 0.377422 & $0.0401 *$ \\
\hline $\mathrm{C} 2$ & $0.33463(11)$ & $0.86695(12)$ & $0.39170(10)$ & $0.0322(5)$ \\
\hline $\mathrm{H} 2$ & 0.386765 & 0.835478 & 0.430202 & $0.0386^{*}$ \\
\hline $\mathrm{C} 3$ & $0.29839(12)$ & $0.81754(12)$ & $0.30656(10)$ & $0.0333(5)$ \\
\hline $\mathrm{H} 3 \mathrm{a}$ & 0.350521 & 0.811391 & 0.27725 & $0.04 *$ \\
\hline $\mathrm{H} 3 \mathrm{~b}$ & 0.250048 & 0.862741 & 0.270514 & $0.04 *$ \\
\hline $\mathrm{C} 4$ & $0.17112(10)$ & $0.71678(11)$ & $0.34935(9)$ & $0.0281(4)$ \\
\hline $\mathrm{H} 4 \mathrm{a}$ & 0.123241 & 0.762704 & 0.313566 & $0.0337^{*}$ \\
\hline $\mathrm{H} 4 \mathrm{~b}$ & 0.189039 & 0.745897 & 0.40767 & $0.0337^{*}$ \\
\hline $\mathrm{C} 5$ & $0.12943(10)$ & $0.61031(11)$ & $0.35226(9)$ & $0.0271(4)$ \\
\hline H5a & 0.07387 & 0.614805 & 0.377337 & $0.0326^{*}$ \\
\hline $\mathrm{H} 5 \mathrm{~b}$ & 0.177161 & 0.564522 & 0.388386 & $0.0326^{*}$ \\
\hline C6 & $0.18571(10)$ & $0.55563(11)$ & $0.23345(9)$ & $0.0262(4)$ \\
\hline H6a & 0.231171 & 0.509606 & 0.271816 & $0.0315^{*}$ \\
\hline $\mathrm{H} 6 \mathrm{~b}$ & 0.168931 & 0.5233 & 0.176272 & $0.0315^{*}$ \\
\hline $\mathrm{C} 7$ & $0.23149(10)$ & $0.65944(12)$ & $0.22789(9)$ & $0.0282(4)$ \\
\hline $\mathrm{H} 7 \mathrm{a}$ & 0.289537 & 0.649813 & 0.207068 & $0.0338^{*}$ \\
\hline $\mathrm{H} 7 \mathrm{~b}$ & 0.187639 & 0.703827 & 0.186493 & $0.0338^{*}$ \\
\hline $\mathrm{C} 8$ & $0.05326(9)$ & $0.46528(10)$ & $0.26756(8)$ & $0.0230(4)$ \\
\hline H8 & 0.099282 & 0.417266 & 0.304542 & $0.0276^{*}$ \\
\hline $\mathrm{C} 11$ & $0.33145(11)$ & $1.00535(12)$ & $0.49876(10)$ & $0.0326(5)$ \\
\hline $\mathrm{C} 12$ & $0.41578(13)$ & $0.97941(14)$ & $0.55685(10)$ & $0.0391(5)$ \\
\hline H12 & 0.454927 & 0.926517 & 0.543205 & $0.047^{*}$ \\
\hline $\mathrm{C} 13$ & $0.44278(15)$ & $1.03053(17)$ & $0.63457(11)$ & $0.0502(6)$ \\
\hline H13 & 0.499799 & 1.011658 & 0.674094 & $0.0603 *$ \\
\hline $\mathrm{C} 14$ & $0.38752(17)$ & $1.10810(17)$ & $0.65451(12)$ & $0.0554(7)$ \\
\hline
\end{tabular}




\begin{tabular}{|c|c|c|c|c|}
\hline $\mathrm{H} 14$ & 0.40688 & 1.143158 & 0.707495 & $0.0665^{*}$ \\
\hline $\mathrm{C} 15$ & $0.30389(15)$ & $1.13552(16)$ & $0.59798(13)$ & $0.0516(7)$ \\
\hline H15 & 0.265849 & 1.18931 & 0.61197 & $0.0619^{*}$ \\
\hline $\mathrm{C} 16$ & $0.27570(13)$ & $1.08400(13)$ & $0.52053(11)$ & $0.0397(5)$ \\
\hline H16 & 0.217882 & 1.102436 & 0.481949 & $0.0476^{*}$ \\
\hline $\mathrm{C} 21$ & $-0.03166(9)$ & $0.47504(10)$ & $0.30647(8)$ & $0.0240(4)$ \\
\hline $\mathrm{C} 22$ & $-0.04272(11)$ & $0.40385(12)$ & $0.36763(10)$ & $0.0315(5)$ \\
\hline $\mathrm{H} 22$ & 0.003276 & 0.351198 & 0.385315 & $0.0378^{*}$ \\
\hline $\mathrm{C} 23$ & $-0.12077(12)$ & $0.40899(13)$ & $0.40333(11)$ & $0.0388(5)$ \\
\hline $\mathrm{H} 23$ & -0.127745 & 0.359826 & 0.445056 & $0.0466^{*}$ \\
\hline $\mathrm{C} 24$ & $-0.18805(12)$ & $0.48544(13)$ & $0.37818(11)$ & $0.0385(5)$ \\
\hline $\mathrm{H} 24$ & -0.241677 & 0.488365 & 0.401911 & $0.0461^{*}$ \\
\hline $\mathrm{C} 25$ & $-0.17687(11)$ & $0.55719(12)$ & $0.31859(11)$ & $0.0351(5)$ \\
\hline $\mathrm{H} 25$ & -0.222711 & 0.610174 & 0.301738 & $0.0421^{*}$ \\
\hline $\mathrm{C} 26$ & $-0.09910(10)$ & $0.55304(11)$ & $0.28271(9)$ & $0.0286(4)$ \\
\hline $\mathrm{H} 26$ & -0.091899 & 0.603396 & 0.241958 & $0.0344^{*}$ \\
\hline $\mathrm{C} 31$ & $0.02520(10)$ & $0.41880(11)$ & $0.17810(9)$ & $0.0248(4)$ \\
\hline $\mathrm{C} 32$ & $0.06690(11)$ & $0.32720(12)$ & $0.16027(10)$ & $0.0331(5)$ \\
\hline H32 & 0.112496 & 0.2938 & 0.204043 & $0.0397 *$ \\
\hline $\mathrm{C} 33$ & $0.04254(13)$ & $0.28416(13)$ & $0.07918(11)$ & $0.0392(5)$ \\
\hline $\mathrm{H} 33$ & 0.071842 & 0.221893 & 0.067798 & $0.047 *$ \\
\hline $\mathrm{C} 34$ & $-0.02351(12)$ & $0.33108(13)$ & $0.01557(10)$ & $0.0379(5)$ \\
\hline H34 & -0.039638 & 0.301886 & -0.039979 & $0.0455^{*}$ \\
\hline $\mathrm{C} 35$ & $-0.06672(12)$ & $0.42142(14)$ & $0.03267(10)$ & 0.0385 \\
\hline $\mathrm{H} 35$ & -0.113709 & 0.453238 & -0.01092 & $0.0461 *$ \\
\hline $\mathrm{C} 36$ & $-0.04162(11)$ & $0.46550(12)$ & $0.11316(10)$ & $0.0321(5)$ \\
\hline H36 & -0.070483 & 0.528311 & 0.123929 & $0.0386^{*}$ \\
\hline $\mathrm{O} 1$ & $0.63327(8)$ & $0.37352(10)$ & $0.53505(8)$ & $0.0392(4)$ \\
\hline $\mathrm{O} 2$ & $0.58197(10)$ & $0.44006(14)$ & $0.67133(9)$ & $0.0614(6)$ \\
\hline $\mathrm{O} 3$ & $0.43608(10)$ & $0.48129(12)$ & $0.65684(9)$ & $0.0549(5)$ \\
\hline $\mathrm{O} 4$ & $0.19464(9)$ & $0.32143(13)$ & $0.44743(10)$ & 0.0569 \\
\hline O5 & $0.22764(11)$ & $0.24248(13)$ & $0.34091(10)$ & $0.0631(6)$ \\
\hline O6 & $0.54745(10)$ & $0.23529(12)$ & $0.30214(8)$ & $0.0518(5)$ \\
\hline O7 & $0.66627(9)$ & $0.28940(12)$ & $0.40564(9)$ & $0.0507(5)$ \\
\hline N3 & $0.49861(10)$ & 0.43835 & $0.63197(9)$ & 0.0379 \\
\hline N4 & $0.25133(10)$ & $0.29121(12)$ & $0.40756(10)$ & $0.0428(5)$ \\
\hline C9 & $0.57563(13)$ & $0.27360(13)$ & $0.37128(12)$ & $0.0398(6)$ \\
\hline C41 & $0.54458(10)$ & $0.35627(11)$ & $0.50735(10)$ & 0.0298 \\
\hline $\mathrm{C} 42$ & $0.50987(11)$ & $0.30564(12)$ & $0.42622(10)$ & 0.0314 \\
\hline $\mathrm{C} 43$ & $0.41587(11)$ & $0.28489(12)$ & $0.39522(10)$ & $0.0329(5)$ \\
\hline $\mathrm{H} 43$ & 0.395087 & 0.249901 & 0.342348 & $0.0394 *$ \\
\hline $\mathrm{C} 44$ & $0.35106(10)$ & $0.31453(12)$ & $0.44039(10)$ & 0.0313 \\
\hline $\mathrm{C} 45$ & $0.37850(10)$ & $0.36477(12)$ & $0.51759(10)$ & $0.0305(4)$ \\
\hline $\mathrm{H} 45$ & 0.332901 & 0.38543 & 0.547596 & $0.0366^{*}$ \\
\hline $\mathrm{C} 46$ & $0.47336(11)$ & $0.38456(11)$ & $0.55054(9)$ & $0.0290(4)$ \\
\hline $\mathrm{H} 7$ & 0.676841 & 0.321683 & 0.458063 & $0.030(4) *$ \\
\hline
\end{tabular}


Atomic displacement parameters $\left(\AA^{2}\right)$

\begin{tabular}{|c|c|c|c|c|c|c|}
\hline & $U^{11}$ & $U^{22}$ & $U^{33}$ & $U^{12}$ & $U^{13}$ & $U^{23}$ \\
\hline N1 & $0.0221(6)$ & $0.0282(6)$ & $0.0236(5)$ & -0.0039 (4) & $0.0055(4)$ & $-0.0028(4)$ \\
\hline $\mathrm{N} 2$ & $0.0209(5)$ & $0.0268(6)$ & $0.0227(5)$ & $-0.0035(4)$ & $0.0063(4)$ & $-0.0032(4)$ \\
\hline $\mathrm{C} 1$ & $0.0300(8)$ & $0.0358(8)$ & $0.0314(7)$ & $-0.0037(6)$ & $0.0017(6)$ & $-0.0003(6)$ \\
\hline $\mathrm{C} 2$ & $0.0301(7)$ & $0.0308(7)$ & $0.0334(7)$ & $-0.0076(6)$ & $0.0033(6)$ & $0.0004(6)$ \\
\hline $\mathrm{C} 3$ & $0.0391(8)$ & $0.0304(7)$ & $0.0318(7)$ & $-0.0113(6)$ & $0.0111(6)$ & $-0.0022(6)$ \\
\hline $\mathrm{C} 4$ & $0.0216(6)$ & $0.0331(7)$ & $0.0302(7)$ & $-0.0026(5)$ & $0.0075(5)$ & $-0.0076(5)$ \\
\hline $\mathrm{C} 5$ & $0.0248(7)$ & $0.0337(7)$ & $0.0241(6)$ & $-0.0051(5)$ & $0.0082(5)$ & $-0.0053(5)$ \\
\hline C6 & $0.0232(6)$ & $0.0304(7)$ & $0.0265(6)$ & $-0.0032(5)$ & 0.0088 & $-0.0047(5)$ \\
\hline $\mathrm{C} 7$ & $0.0285(7)$ & $0.0344(7)$ & $0.0223(6)$ & $-0.0060(6)$ & $0.0073(5)$ & $-0.0045(5)$ \\
\hline $\mathrm{C} 8$ & $0.0197(6)$ & $0.0247(6)$ & $0.0245(6)$ & $0.0003(5)$ & $0.0054(5)$ & 0.0010 \\
\hline C11 & $0.0357(8)$ & $0.0306(7)$ & $0.0307(7)$ & $-0.0080(6)$ & $0.0064(6)$ & $0.0006(6)$ \\
\hline $\mathrm{C} 12$ & $0.0398(9)$ & $0.0415(9)$ & $0.0337(8)$ & $-0.0067(7)$ & $0.0042(7)$ & $0.0007(7)$ \\
\hline C13 & $0.0523(11)$ & $0.0604(12)$ & $0.0319(8)$ & $-0.0142(9)$ & $-0.0018(8)$ & $0.0005(8)$ \\
\hline C14 & $0.0691(14)$ & $0.0629(13)$ & $0.0354(9)$ & $-0.0230(10)$ & $0.0149(9)$ & $-0.0159(8)$ \\
\hline C15 & $0.0584(12)$ & $0.0455(10)$ & $0.0562(11)$ & $-0.0106(9)$ & $0.0242(10)$ & $-0.0151(8)$ \\
\hline $\mathrm{C} 16$ & 0.0392 (9) & $0.0350(8)$ & $0.0444(9)$ & $-0.0049(7)$ & $0.0090(7)$ & $-0.0039(7)$ \\
\hline $\mathrm{C} 21$ & $0.0213(6)$ & $0.0250(6)$ & $0.0255(6)$ & -0.0020 & $0.0050(5)$ & $-0.0024(5)$ \\
\hline $\mathrm{C} 22$ & $0.0311(7)$ & $0.0329(8)$ & $0.0325(7)$ & $0.0030(6)$ & $0.0114(6)$ & $0.0046(6)$ \\
\hline $\mathrm{C} 23$ & $0.0426(9)$ & $0.0390(9)$ & $0.0413(9)$ & $0.0005(7)$ & $0.0228(7)$ & $0.0058(7)$ \\
\hline $\mathrm{C} 24$ & $0.0344(8)$ & $0.0391(8)$ & $0.0493(9)$ & $-0.0028(6)$ & $0.0245(7)$ & $-0.0078(7)$ \\
\hline $\mathrm{C} 25$ & $0.0278(7)$ & $0.0336(8)$ & $0.0447(9)$ & $0.0044(6)$ & $0.0104(6)$ & $-0.0058(6)$ \\
\hline $\mathrm{C} 26$ & $0.0269(7)$ & $0.0271(7)$ & $0.0323(7)$ & $0.0015(5)$ & $0.0079(6)$ & $0.0002(5)$ \\
\hline $\mathrm{C} 31$ & $0.0226(6)$ & $0.0253(6)$ & $0.0282(6)$ & $-0.0049(5)$ & $0.0093(5)$ & $-0.0016(5)$ \\
\hline $\mathrm{C} 32$ & $0.0327(8)$ & $0.0296(7)$ & $0.0367(8)$ & $0.0017(6)$ & $0.0076(6)$ & $-0.0021(6)$ \\
\hline C33 & $0.0437(9)$ & $0.0299(8)$ & $0.0463(9)$ & $-0.0014(7)$ & $0.0154(8)$ & $-0.0097(7)$ \\
\hline C34 & $0.0452(9)$ & $0.0381(9)$ & $0.0313(8)$ & $-0.0104(7)$ & $0.0109(7)$ & $-0.0090(6)$ \\
\hline $\mathrm{C} 35$ & $0.0387(9)$ & $0.0451(9)$ & $0.0283(7)$ & $-0.0015(7)$ & $0.0017(7)$ & $-0.0024(7)$ \\
\hline $\mathrm{C} 36$ & $0.0316(8)$ & $0.0337(8)$ & $0.0310(7)$ & $0.0020(6)$ & $0.0073(6)$ & $-0.0020(6)$ \\
\hline $\mathrm{O} 1$ & $0.0242(6)$ & $0.0466(7)$ & $0.0449(6)$ & $-0.0022(5)$ & $0.0046(5)$ & $0.0065(5)$ \\
\hline $\mathrm{O} 2$ & $0.0364(7)$ & $0.0954(12)$ & $0.0457(8)$ & $0.0021(7)$ & $-0.0029(6)$ & $-0.0243(7)$ \\
\hline $\mathrm{O} 3$ & $0.0459(8)$ & $0.0698(9)$ & $0.0453(7)$ & $0.0153(7)$ & $0.0038(6)$ & $-0.0171(6)$ \\
\hline $\mathrm{O} 4$ & $0.0239(6)$ & $0.0732(10)$ & $0.0713(9)$ & $0.0049(6)$ & $0.0067(6)$ & $-0.0008(8)$ \\
\hline O5 & $0.0465(8)$ & $0.0774(11)$ & $0.0531(8)$ & $-0.0103(7)$ & $-0.0120(7)$ & $-0.0150(7)$ \\
\hline O6 & 0.0595 (9) & $0.0612(9)$ & $0.0400(7)$ & $0.0066(7)$ & $0.0226(6)$ & $-0.0026(6)$ \\
\hline $\mathrm{O} 7$ & $0.0391(7)$ & $0.0619(9)$ & $0.0567(8)$ & $0.0007(6)$ & $0.0223(6)$ & $-0.0035(6)$ \\
\hline N3 & $0.0355(7)$ & $0.0405(8)$ & $0.0347(7)$ & $0.0038(6)$ & $0.0023(6)$ & $-0.0023(6)$ \\
\hline N4 & $0.0303(7)$ & $0.0466(8)$ & $0.0442(8)$ & $-0.0012(6)$ & $-0.0056(6)$ & $0.0040(6)$ \\
\hline C9 & $0.0393(9)$ & $0.0352(8)$ & $0.0491(10)$ & $0.0046(7)$ & $0.0190(8)$ & $0.0095(7)$ \\
\hline $\mathrm{C} 41$ & $0.0232(7)$ & $0.0277(7)$ & $0.0368(7)$ & $0.0028(5)$ & $0.0038(6)$ & $0.0099(6)$ \\
\hline $\mathrm{C} 42$ & $0.0330(8)$ & $0.0293(7)$ & $0.0345(7)$ & $0.0049(6)$ & $0.0133(6)$ & $0.0074(6)$ \\
\hline $\mathrm{C} 43$ & $0.0370(8)$ & $0.0322(8)$ & $0.0284(7)$ & $0.0011(6)$ & $0.0059(6)$ & $0.0030(6)$ \\
\hline $\mathrm{C} 44$ & $0.0238(7)$ & $0.0334(8)$ & $0.0333(7)$ & $0.0012(5)$ & $0.0000(6)$ & $0.0037(6)$ \\
\hline $\mathrm{C} 45$ & $0.0255(7)$ & $0.0325(7)$ & $0.0333(7)$ & $0.0060(6)$ & $0.0067(6)$ & $0.0030(6)$ \\
\hline $\mathrm{C} 46$ & $0.0282(7)$ & $0.0282(7)$ & $0.0282(7)$ & 0.0018 & $0.0017(6)$ & $0.0016(5)$ \\
\hline
\end{tabular}


Geometric parameters $\left(\AA,{ }^{o}\right)$

\begin{tabular}{|c|c|c|c|}
\hline $\mathrm{N} 1-\mathrm{H} 71$ & $0.9448(11)$ & $\mathrm{C} 16-\mathrm{H} 16$ & 0.95 \\
\hline $\mathrm{N} 1-\mathrm{C} 3$ & $1.5141(19)$ & $\mathrm{C} 21-\mathrm{C} 22$ & $1.388(2)$ \\
\hline $\mathrm{N} 1-\mathrm{C} 4$ & $1.492(2)$ & $\mathrm{C} 21-\mathrm{C} 26$ & $1.3962(19)$ \\
\hline $\mathrm{N} 1-\mathrm{C} 7$ & $1.5036(18)$ & $\mathrm{C} 22-\mathrm{H} 22$ & 0.95 \\
\hline $\mathrm{N} 2-\mathrm{C} 5$ & $1.4718(17)$ & $\mathrm{C} 22-\mathrm{C} 23$ & $1.395(3)$ \\
\hline $\mathrm{N} 2-\mathrm{C} 6$ & $1.4682(19)$ & $\mathrm{C} 23-\mathrm{H} 23$ & 0.95 \\
\hline $\mathrm{N} 2-\mathrm{C} 8$ & $1.4848(17)$ & $\mathrm{C} 23-\mathrm{C} 24$ & $1.383(2)$ \\
\hline $\mathrm{C} 1-\mathrm{H} 1 \mathrm{a}$ & 0.95 & $\mathrm{C} 24-\mathrm{H} 24$ & 0.95 \\
\hline $\mathrm{C} 1-\mathrm{C} 2$ & $1.317(2)$ & $\mathrm{C} 24-\mathrm{C} 25$ & $1.375(3)$ \\
\hline $\mathrm{C} 1-\mathrm{C} 11$ & $1.474(2)$ & $\mathrm{C} 25-\mathrm{H} 25$ & 0.95 \\
\hline $\mathrm{C} 2-\mathrm{H} 2$ & 0.95 & $\mathrm{C} 25-\mathrm{C} 26$ & $1.392(2)$ \\
\hline $\mathrm{C} 2-\mathrm{C} 3$ & $1.494(2)$ & $\mathrm{C} 26-\mathrm{H} 26$ & 0.95 \\
\hline $\mathrm{C} 3-\mathrm{H} 3 \mathrm{a}$ & 0.99 & $\mathrm{C} 31-\mathrm{C} 32$ & $1.393(2)$ \\
\hline $\mathrm{C} 3-\mathrm{H} 3 \mathrm{~b}$ & 0.99 & $\mathrm{C} 31-\mathrm{C} 36$ & 1.3867 (19) \\
\hline $\mathrm{H} 3 \mathrm{a}-\mathrm{H} 3 \mathrm{~b}$ & 1.5869 & C32-H32 & 0.95 \\
\hline $\mathrm{C} 4-\mathrm{H} 4 \mathrm{a}$ & 0.99 & $\mathrm{C} 32-\mathrm{C} 33$ & $1.389(2)$ \\
\hline $\mathrm{C} 4-\mathrm{H} 4 \mathrm{~b}$ & 0.99 & $\mathrm{C} 33-\mathrm{H} 33$ & 0.95 \\
\hline $\mathrm{C} 4-\mathrm{C} 5$ & $1.511(2)$ & $\mathrm{C} 33-\mathrm{C} 34$ & $1.370(2)$ \\
\hline $\mathrm{H} 4 \mathrm{a}-\mathrm{H} 4 \mathrm{~b}$ & 1.6058 & C34-H34 & 0.95 \\
\hline $\mathrm{C} 5-\mathrm{H} 5 \mathrm{a}$ & 0.99 & $\mathrm{C} 34-\mathrm{C} 35$ & $1.386(3)$ \\
\hline $\mathrm{C} 5-\mathrm{H} 5 \mathrm{~b}$ & 0.99 & C35-H35 & 0.95 \\
\hline $\mathrm{H} 5 \mathrm{a}-\mathrm{H} 5 \mathrm{~b}$ & 1.6091 & $\mathrm{C} 35-\mathrm{C} 36$ & $1.387(2)$ \\
\hline C6-H6a & 0.99 & C36- & 0.95 \\
\hline $\mathrm{C} 6-\mathrm{H} 6 \mathrm{~b}$ & 0.99 & $\mathrm{O} 1-\mathrm{C} 41$ & $1.2810(17)$ \\
\hline $\mathrm{C} 6-\mathrm{C} 7$ & $1.512(2)$ & $\mathrm{O} 2-\mathrm{N} 3$ & $1.2281(18)$ \\
\hline $\mathrm{H} 6 \mathrm{a}-\mathrm{H} 6 \mathrm{~b}$ & 1.6016 & $\mathrm{O} 3-\mathrm{N} 3$ & $1.215(2)$ \\
\hline $\mathrm{C} 7-\mathrm{H} 7 \mathrm{a}$ & 0.99 & $\mathrm{O} 4-\mathrm{N} 4$ & $1.227(2)$ \\
\hline $\mathrm{C} 7-\mathrm{H} 7 \mathrm{~b}$ & 0.99 & $\mathrm{O} 5-\mathrm{N} 4$ & $1.224(2)$ \\
\hline $\mathrm{H} 7 \mathrm{a}-\mathrm{H} 7 \mathrm{~b}$ & 1.6014 & $\mathrm{O} 6-\mathrm{C} 9$ & $1.201(2)$ \\
\hline $\mathrm{C} 8-\mathrm{H} 8$ & 1 & $\mathrm{O} 7-\mathrm{C} 9$ & $1.319(2)$ \\
\hline $\mathrm{C} 8-\mathrm{C} 21$ & $1.522(2)$ & $\mathrm{O} 7-\mathrm{H} 7$ & $0.9238(15)$ \\
\hline $\mathrm{C} 8-\mathrm{C} 31$ & $1.5282(19)$ & $\mathrm{N} 3-\mathrm{C} 46$ & $1.456(2)$ \\
\hline $\mathrm{C} 11-\mathrm{C} 12$ & $1.396(2)$ & $\mathrm{N} 4-\mathrm{C} 44$ & $1.453(2)$ \\
\hline $\mathrm{C} 11-\mathrm{C} 16$ & $1.399(3)$ & $\mathrm{C} 9-\mathrm{C} 42$ & $1.512(3)$ \\
\hline $\mathrm{C} 12-\mathrm{H} 12$ & 0.95 & $\mathrm{C} 41-\mathrm{C} 42$ & $1.444(2)$ \\
\hline $\mathrm{C} 12-\mathrm{C} 13$ & $1.390(2)$ & $\mathrm{C} 41-\mathrm{C} 46$ & $1.430(2)$ \\
\hline C13-H13 & 0.95 & $\mathrm{C} 42-\mathrm{C} 43$ & $1.367(2)$ \\
\hline $\mathrm{C} 13-\mathrm{C} 14$ & $1.372(3)$ & $\mathrm{C} 43-\mathrm{H} 43$ & 0.95 \\
\hline C14-H14 & 0.95 & $\mathrm{C} 43-\mathrm{C} 44$ & $1.379(2)$ \\
\hline $\mathrm{C} 14-\mathrm{C} 15$ & $1.382(3)$ & $\mathrm{C} 44-\mathrm{C} 45$ & $1.378(2)$ \\
\hline C15-H15 & 0.95 & $\mathrm{C} 45-\mathrm{H} 45$ & 0.95 \\
\hline $\mathrm{C} 15-\mathrm{C} 16$ & $1.390(3)$ & $\mathrm{C} 45-\mathrm{C} 46$ & $1.380(2)$ \\
\hline $\mathrm{H} 71-\mathrm{N} 1-\mathrm{C} 3$ & $109.70(10)$ & $\mathrm{C} 15-\mathrm{C} 16-\mathrm{H} 16$ & 119.61 \\
\hline $\mathrm{H} 71-\mathrm{N} 1-\mathrm{C} 4$ & $107.37(11)$ & $\mathrm{C} 8-\mathrm{C} 21-\mathrm{C} 22$ & $118.95(12)$ \\
\hline $\mathrm{H} 71-\mathrm{N} 1-\mathrm{C} 7$ & $106.90(11)$ & $\mathrm{C} 8-\mathrm{C} 21-\mathrm{C} 26$ & $122.30(13)$ \\
\hline
\end{tabular}




\begin{tabular}{|c|c|c|c|}
\hline $\mathrm{C} 3-\mathrm{N} 1-\mathrm{C} 4$ & $112.27(12)$ & $\mathrm{C} 22-\mathrm{C} 21-\mathrm{C} 26$ & $118.75(14)$ \\
\hline $\mathrm{C} 3-\mathrm{N} 1-\mathrm{C} 7$ & $110.59(11)$ & $\mathrm{C} 21-\mathrm{C} 22-\mathrm{H} 22$ & 119.72 \\
\hline $\mathrm{C} 4-\mathrm{N} 1-\mathrm{C} 7$ & $109.82(10)$ & $\mathrm{C} 21-\mathrm{C} 22-\mathrm{C} 23$ & $120.56(14)$ \\
\hline $\mathrm{C} 5-\mathrm{N} 2-\mathrm{C} 6$ & $107.42(10)$ & $\mathrm{H} 22-\mathrm{C} 22-\mathrm{C} 23$ & 119.72 \\
\hline $\mathrm{C} 5-\mathrm{N} 2-\mathrm{C} 8$ & $110.41(11)$ & $\mathrm{C} 22-\mathrm{C} 23-\mathrm{H} 23$ & 119.9 \\
\hline $\mathrm{C} 6-\mathrm{N} 2-\mathrm{C} 8$ & $110.71(11)$ & $\mathrm{C} 22-\mathrm{C} 23-\mathrm{C} 24$ & $120.20(16)$ \\
\hline $\mathrm{H} 1 \mathrm{a}-\mathrm{C} 1-\mathrm{C} 2$ & 116.52 & $\mathrm{H} 23-\mathrm{C} 23-\mathrm{C} 24$ & 119.9 \\
\hline $\mathrm{H} 1 \mathrm{a}-\mathrm{C} 1-\mathrm{C} 11$ & 116.52 & $\mathrm{C} 23-\mathrm{C} 24-\mathrm{H} 24$ & 120.2 \\
\hline $\mathrm{C} 2-\mathrm{C} 1-\mathrm{C} 11$ & $126.96(14)$ & $\mathrm{C} 23-\mathrm{C} 24-\mathrm{C} 25$ & $119.60(17)$ \\
\hline $\mathrm{C} 1-\mathrm{C} 2-\mathrm{H} 2$ & 118.03 & $\mathrm{H} 24-\mathrm{C} 24-\mathrm{C} 25$ & 120.2 \\
\hline $\mathrm{C} 1-\mathrm{C} 2-\mathrm{C} 3$ & $123.95(13)$ & $\mathrm{C} 24-\mathrm{C} 25-\mathrm{H} 25$ & 119.64 \\
\hline $\mathrm{H} 2-\mathrm{C} 2-\mathrm{C} 3$ & 118.03 & $\mathrm{C} 24-\mathrm{C} 25-\mathrm{C} 26$ & $120.72(15)$ \\
\hline $\mathrm{N} 1-\mathrm{C} 3-\mathrm{C} 2$ & $112.26(13)$ & $\mathrm{H} 25-\mathrm{C} 25-\mathrm{C} 26$ & 119.64 \\
\hline $\mathrm{N} 1-\mathrm{C} 3-\mathrm{H} 3 \mathrm{a}$ & 109.47 & $\mathrm{C} 21-\mathrm{C} 26-\mathrm{C} 25$ & $120.16(14)$ \\
\hline $\mathrm{N} 1-\mathrm{C} 3-\mathrm{H} 3 \mathrm{~b}$ & 109.47 & $\mathrm{C} 21-\mathrm{C} 26-\mathrm{H} 26$ & 119.92 \\
\hline $\mathrm{C} 2-\mathrm{C} 3-\mathrm{H} 3 \mathrm{a}$ & 109.47 & $\mathrm{C} 25-\mathrm{C} 26-\mathrm{H} 26$ & 119.92 \\
\hline $\mathrm{C} 2-\mathrm{C} 3-\mathrm{H} 3 \mathrm{~b}$ & 109.47 & $\mathrm{C} 8-\mathrm{C} 31-\mathrm{C} 32$ & $119.98(11)$ \\
\hline $\mathrm{H} 3 \mathrm{a}-\mathrm{C} 3-\mathrm{H} 3 \mathrm{~b}$ & 106.54 & $\mathrm{C} 8-\mathrm{C} 31-\mathrm{C} 36$ & $121.61(13)$ \\
\hline $\mathrm{N} 1-\mathrm{C} 4-\mathrm{H} 4 \mathrm{a}$ & 109.47 & $\mathrm{C} 32-\mathrm{C} 31-\mathrm{C} 36$ & $118.41(13)$ \\
\hline $\mathrm{N} 1-\mathrm{C} 4-\mathrm{H} 4 \mathrm{~b}$ & 109.47 & $\mathrm{C} 31-\mathrm{C} 32-\mathrm{H} 32$ & 119.64 \\
\hline $\mathrm{N} 1-\mathrm{C} 4-\mathrm{C} 5$ & $110.53(12)$ & $\mathrm{C} 31-\mathrm{C} 32-\mathrm{C} 33$ & $120.73(13)$ \\
\hline $\mathrm{H} 4 \mathrm{a}-\mathrm{C} 4-\mathrm{H} 4 \mathrm{~b}$ & 108.39 & $\mathrm{H} 32-\mathrm{C} 32-\mathrm{C} 33$ & 119.64 \\
\hline $\mathrm{H} 4 \mathrm{a}-\mathrm{C} 4-\mathrm{C} 5$ & 109.47 & $\mathrm{C} 32-\mathrm{C} 33-\mathrm{H} 33$ & 119.85 \\
\hline $\mathrm{H} 4 \mathrm{~b}-\mathrm{C} 4-\mathrm{C} 5$ & 109.47 & $\mathrm{C} 32-\mathrm{C} 33-\mathrm{C} 34$ & $120.30(16)$ \\
\hline $\mathrm{N} 2-\mathrm{C} 5-\mathrm{C} 4$ & $110.21(12)$ & $\mathrm{H} 33-\mathrm{C} 33-\mathrm{C} 34$ & 119.85 \\
\hline $\mathrm{N} 2-\mathrm{C} 5-\mathrm{H} 5 \mathrm{a}$ & 109.47 & $\mathrm{C} 33-\mathrm{C} 34-\mathrm{H} 34$ & 120.18 \\
\hline $\mathrm{N} 2-\mathrm{C} 5-\mathrm{H} 5 \mathrm{~b}$ & 109.47 & $\mathrm{C} 33-\mathrm{C} 34-\mathrm{C} 35$ & $119.65(15)$ \\
\hline $\mathrm{C} 4-\mathrm{C} 5-\mathrm{H} 5 \mathrm{a}$ & 109.47 & $\mathrm{H} 34-\mathrm{C} 34-\mathrm{C} 35$ & 120.18 \\
\hline $\mathrm{C} 4-\mathrm{C} 5-\mathrm{H} 5 \mathrm{~b}$ & 109.47 & $\mathrm{C} 34-\mathrm{C} 35-\mathrm{H} 35$ & 119.87 \\
\hline $\mathrm{H} 5 \mathrm{a}-\mathrm{C} 5-\mathrm{H} 5 \mathrm{~b}$ & 108.72 & $\mathrm{C} 34-\mathrm{C} 35-\mathrm{C} 36$ & $120.25(14)$ \\
\hline $\mathrm{N} 2-\mathrm{C} 6-\mathrm{H} 6 \mathrm{a}$ & 109.47 & $\mathrm{H} 35-\mathrm{C} 35-\mathrm{C} 36$ & 119.88 \\
\hline $\mathrm{N} 2-\mathrm{C} 6-\mathrm{H} 6 \mathrm{~b}$ & 109.47 & $\mathrm{C} 31-\mathrm{C} 36-\mathrm{C} 35$ & $120.65(15)$ \\
\hline $\mathrm{N} 2-\mathrm{C} 6-\mathrm{C} 7$ & $110.93(12)$ & $\mathrm{C} 31-\mathrm{C} 36-\mathrm{H} 36$ & 119.67 \\
\hline $\mathrm{H} 6 \mathrm{a}-\mathrm{C} 6-\mathrm{H} 6 \mathrm{~b}$ & 107.97 & $\mathrm{C} 35-\mathrm{C} 36-\mathrm{H} 36$ & 119.68 \\
\hline $\mathrm{H} 6 \mathrm{a}-\mathrm{C} 6-\mathrm{C} 7$ & 109.47 & $\mathrm{C} 41-\mathrm{O} 1-\mathrm{H} 7$ & $101.64(10)$ \\
\hline $\mathrm{H} 6 \mathrm{~b}-\mathrm{C} 6-\mathrm{C} 7$ & 109.47 & $\mathrm{C} 9-\mathrm{O} 7-\mathrm{H} 7$ & $112.68(16)$ \\
\hline $\mathrm{N} 1-\mathrm{C} 7-\mathrm{C} 6$ & $110.95(12)$ & $\mathrm{O} 2-\mathrm{N} 3-\mathrm{O} 3$ & $123.16(15)$ \\
\hline $\mathrm{N} 1-\mathrm{C} 7-\mathrm{H} 7 \mathrm{a}$ & 109.47 & $\mathrm{O} 2-\mathrm{N} 3-\mathrm{C} 46$ & $118.72(15)$ \\
\hline $\mathrm{N} 1-\mathrm{C} 7-\mathrm{H} 7 \mathrm{~b}$ & 109.47 & $\mathrm{O} 3-\mathrm{N} 3-\mathrm{C} 46$ & $118.10(13)$ \\
\hline $\mathrm{C} 6-\mathrm{C} 7-\mathrm{H} 7 \mathrm{a}$ & 109.47 & $\mathrm{O} 4-\mathrm{N} 4-\mathrm{O} 5$ & $122.91(15)$ \\
\hline $\mathrm{C} 6-\mathrm{C} 7-\mathrm{H} 7 \mathrm{~b}$ & 109.47 & $\mathrm{O} 4-\mathrm{N} 4-\mathrm{C} 44$ & $118.79(14)$ \\
\hline $\mathrm{H} 7 \mathrm{a}-\mathrm{C} 7-\mathrm{H} 7 \mathrm{~b}$ & 107.95 & $\mathrm{O} 5-\mathrm{N} 4-\mathrm{C} 44$ & $118.29(16)$ \\
\hline $\mathrm{N} 2-\mathrm{C} 8-\mathrm{H} 8$ & 108.39 & $\mathrm{O} 6-\mathrm{C} 9-\mathrm{O} 7$ & $122.64(19)$ \\
\hline $\mathrm{N} 2-\mathrm{C} 8-\mathrm{C} 21$ & $111.04(11)$ & $\mathrm{O} 6-\mathrm{C} 9-\mathrm{C} 42$ & $122.44(16)$ \\
\hline $\mathrm{N} 2-\mathrm{C} 8-\mathrm{C} 31$ & $110.45(11)$ & $\mathrm{O} 7-\mathrm{C} 9-\mathrm{C} 42$ & $114.91(15)$ \\
\hline $\mathrm{H} 8-\mathrm{C} 8-\mathrm{C} 21$ & 107.41 & $\mathrm{O} 1-\mathrm{C} 41-\mathrm{C} 42$ & $120.00(15)$ \\
\hline $\mathrm{H} 8-\mathrm{C} 8-\mathrm{C} 31$ & 108.03 & $\mathrm{O} 1-\mathrm{C} 41-\mathrm{C} 46$ & $125.00(14)$ \\
\hline $\mathrm{C} 21-\mathrm{C} 8-\mathrm{C} 31$ & $111.38(10)$ & $\mathrm{C} 42-\mathrm{C} 41-\mathrm{C} 46$ & $115.00(13)$ \\
\hline
\end{tabular}




$\begin{array}{ll}\mathrm{C} 1-\mathrm{C} 11-\mathrm{C} 12 & 122.33(15) \\ \mathrm{C} 1-\mathrm{C} 11-\mathrm{C} 16 & 119.24(13) \\ \mathrm{C} 12-\mathrm{C} 11-\mathrm{C} 16 & 118.42(15) \\ \mathrm{C} 11-\mathrm{C} 12-\mathrm{H} 12 & 119.81 \\ \mathrm{C} 11-\mathrm{C} 12-\mathrm{C} 13 & 120.37(17) \\ \mathrm{H} 12-\mathrm{C} 12-\mathrm{C} 13 & 119.81 \\ \mathrm{C} 12-\mathrm{C} 13-\mathrm{H} 13 & 119.82 \\ \mathrm{C} 12-\mathrm{C} 13-\mathrm{C} 14 & 120.37(17) \\ \mathrm{H} 13-\mathrm{C} 13-\mathrm{C} 14 & 119.82 \\ \mathrm{C} 13-\mathrm{C} 14-\mathrm{H} 14 & 119.8 \\ \mathrm{C} 13-\mathrm{C} 14-\mathrm{C} 15 & 120.40(18) \\ \mathrm{H} 14-\mathrm{C} 14-\mathrm{C} 15 & 119.8 \\ \mathrm{C} 14-\mathrm{C} 15-\mathrm{H} 15 & 120.17 \\ \mathrm{C} 14-\mathrm{C} 15-\mathrm{C} 16 & 119.7(2) \\ \mathrm{H} 15-\mathrm{C} 15-\mathrm{C} 16 & 120.17 \\ \mathrm{C} 11-\mathrm{C} 16-\mathrm{C} 15 & 120.77(16) \\ \mathrm{C} 11-\mathrm{C} 16-\mathrm{H} 16 & 119.61\end{array}$

$121.64(14)$

$116.80(14)$

121.55 (16)

119.87

120.27 (14)

119.87

120.01 (14)

$118.40(15)$

$121.59(13)$

120.57

$118.86(15)$

120.57

120.55 (13)

$116.73(14)$

$122.71(13)$

$148.96(9)$

Hydrogen-bond geometry $\left(A,{ }^{\circ}\right)$

\begin{tabular}{lllll}
\hline$D-\mathrm{H} \cdots A$ & $D-\mathrm{H}$ & $\mathrm{H} \cdots A$ & $D \cdots A$ & $D-\mathrm{H} \cdots A$ \\
\hline $\mathrm{N} 1-\mathrm{H} 71 \cdots \mathrm{O} 1^{\mathrm{i}}$ & $0.9448(11)$ & $1.9544(11)$ & $2.8126(15)$ & $150.01(8)$ \\
$\mathrm{N} 1-\mathrm{H} 71 \cdots \mathrm{O} 2^{\mathrm{i}}$ & $0.9448(11)$ & $2.3020(16)$ & $3.032(2)$ & $133.62(8)$ \\
$\mathrm{C} 3-\mathrm{H} 3 a \cdots 6^{\mathrm{ii}}$ & 0.99 & 2.40 & $3.340(2)$ & 159 \\
$\mathrm{C} 5-\mathrm{H} 5 a \cdots \mathrm{C} 21$ & 0.99 & 2.47 & $2.8777(19)$ & 104 \\
$\mathrm{C} 6-\mathrm{H} 6 b \cdots \mathrm{C} 31$ & 0.99 & 2.50 & $2.8974(19)$ & 104 \\
$\mathrm{O} 7-\mathrm{H} 7 \cdots \mathrm{O} 1$ & $0.9238(15)$ & $1.6675(13)$ & $2.505(2)$ & $148.96(9)$ \\
$\mathrm{O} 7-\mathrm{H} 7 \cdots \mathrm{C} 41$ & $0.9238(15)$ & $2.2985(16)$ & $2.827(2)$ & $115.91(9)$ \\
\hline
\end{tabular}

Symmetry codes: (i) $-x+1,-y+1,-z+1$; (ii) $-x+1, y+1 / 2,-z+1 / 2$. 